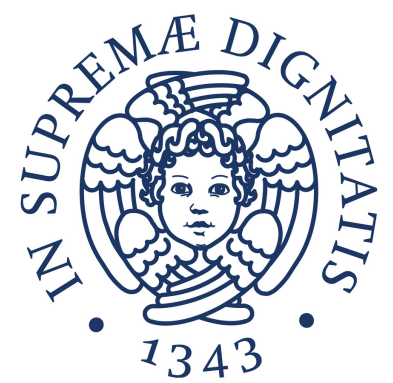

Università degli Studi di PisA

Facoltà di scienze matematiche, fisiche e naturali Corso di Laurea Magistrale in Fisica

\title{
Studies of the impact of magnetic field uncertainties on physics parameters of the Mu2e experiment
}

Advisors:

Prof. Giorgio Bellettini

Dr. Costas Vellidis
Candidate:

Federica Bradascio 

"It is a capital mistake to theorize before one has data. Insensibly one begins to twist facts to suit theories, instead of theories to suit facts." Arthur Conan Doyle 

Al mio babbo, mio modello di razionalità e bontà.

Alla mia mamma, per me esempio di forza e dolcezza.

Alla mia sorellina, mia inesauribile fonte di felicità e gioia. 



\section{ACKNOWLEDGEMENTS}

I would like to say Grazie to:

Prof. Giorgio Bellettini, who gave me the possibility to make this thesis at Fermilab and for always being a point of reference in a foreign place;

Dr. Costas Vellidis, for being a mentor and a friend, besides a supervisor, in these months;

Prof. Simone Donati and Dr. Emanuela Barzi, for encouraging and supporting me in my work at Fermilab;

Dr. Doug Glenzinski and Prof. Jim Miller, for supporting and making me feel part of the Mu2e experiment;

Dr. Robert Kutschke and Dr. Andrei Gaponenko, for their help in the complex world of simulations and FHiCL files;

Dr. Mauricio Lopes, for his suggestions, intuitions and help in the field of solenoid magnets;

My family, for always being my Home, no matter in which country I live;

You, all you, for giving me great moments and making me laugh when everything else appeared grey. 


\section{Abstract}

The Mu2e experiment at Fermilab will search for a signature of charged lepton flavor violation, an effect prohibitively too small to be observed within the Standard Model of particle physics. Therefore, its observation is a signal of new physics. The signature that Mu2e will search for is the ratio of the rate of neutrinoless coherent conversion of muons into electrons in the field of a nucleus, relative to the muon capture rate by the nucleus. The conversion process is an example of charged lepton flavor violation. This experiment aims at a sensitivity of four orders of magnitude higher than previous related experiments. The desired sensitivity implies highly demanding requirements of accuracy in the design and conduct of the experiment. It is therefore important to investigate the tolerance of the experiment to instrumental uncertainties and provide specifications that the design and construction must meet. This is the core of the work reported in this thesis.

The design of the experiment is based on three superconducting solenoid magnets. The most important uncertainties in the magnetic field of the solenoids can arise from misalignments of the Transport Solenoid, which transfers the beam from the muon production area to the detector area and eliminates beam-originating backgrounds. In this thesis, the field uncertainties induced by possible misalignments and their impact on the physics parameters of the experiment are examined. The physics parameters include the muon and pion stopping rates and the scattering of beam electrons off the capture target, which determine the signal, intrinsic background and late-arriving background yields, respectively. Additionally, a possible test of the Transport Solenoid alignment with low momentum electrons is examined, as an alternative option to measure its field with conventional probes, which is technically difficult due to mechanical interference.

Misalignments of the Transport Solenoid were simulated using standard magnetic field calculation tools. Particle transport was simulated using the Mu2e Offline software, which includes realistic models of particle interactions with materials in the full Mu2e geometry. The physics parameters were found tolerant within the precision requirements of the experiment for rigid-body type of misalignments, which are the most dangerous, up to a maximum coil displacement of nearly $10 \mathrm{~mm}$. With the appropriate choice of low momentum electron detector, the proposed Transport Solenoid test is found to be sensitive to such misalignments. 


\section{CONTENTS}

Abstract

1 Introduction 1

1.1 The Mu2e experiment $\ldots \ldots \ldots \ldots \ldots \ldots \ldots \ldots \ldots$

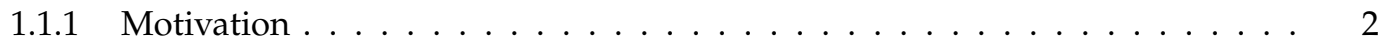

1.1.2 Present status of CLFV searches using muons . . . . . . . . . . . 4

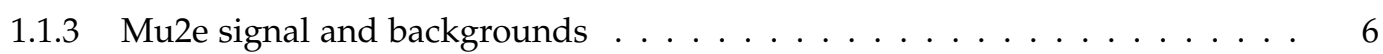

1.1.4 Muze instrumentation . . . . . . . . . . . . . . . . . . . . . . . 10

1.2 Motion of charged particles in a solenoidal magnetic field . . . . . . . . . . . . 13

1.3 The Mu2e solenoid magnets . . . . . . . . . . . . . . . . . . . . 14

1.3.1 Mu2e coordinate Systems . . . . . . . . . . . . . . . . . . . 15

1.3.2 Production and Detector Solenoids . . . . . . . . . . . . . . 16

1.3.3 Transport Solenoid . . . . . . . . . . . . . . . . . . . . . . . . . 17

1.4 Sources of uncertainty in the Mu2e magnetic fields . . . . . . . . . . . . 18

1.5 The Mu2e simulation package . . . . . . . . . . . . . . . . . . . . . . 19

1.5.1 ART and ROOT output files . . . . . . . . . . . . . . . 19

1.5.2 Stage 1,2 and 3 simulations . . . . . . . . . . . . . . 20

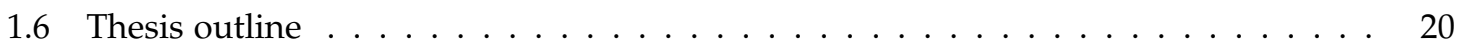

2 Magnetic field calculations 23

2.1 Mu2e field maps . . . . . . . . . . . . . . . . . . 23

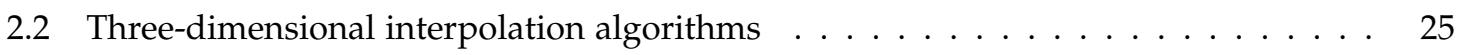

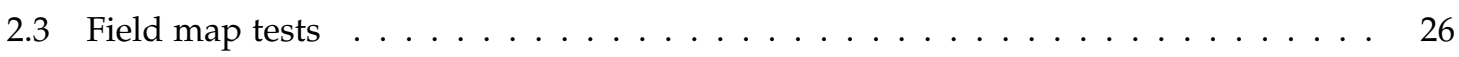

2.3.1 Map validations . . . . . . . . . . . . . . . . . . . . . . 26

2.3 .2 Algorithm tests . . . . . . . . . . . . . . . . . . . . . 30

2.4 Field map calculations using MATLAB $\ldots \ldots \ldots \ldots \ldots \ldots \ldots \ldots \ldots \ldots \ldots$

2.4 .1 The SolCalc package . . . . . . . . . . . . . . . . . . . . . 32

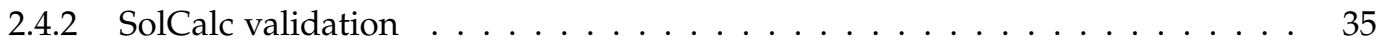




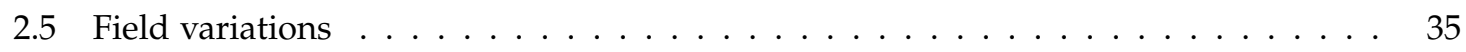

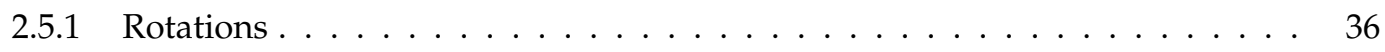

2.5.2 Parallel coil displacements . . . . . . . . . . . . . . 38

3 Muon and pion stopping rate studies $\quad 43$

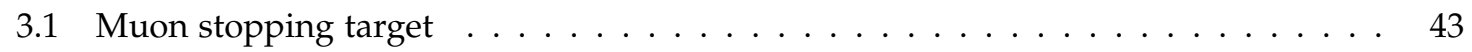

3.2 Stopped particle simulations $\ldots \ldots \ldots \ldots \ldots \ldots \ldots \ldots$

3.3 Results . . . . . . . . . . . . . . . . . . . . . 48

3.3.1 Rotations . . . . . . . . . . . . . . . . . . . . . . . 48

3.3.2 Parallel coil displacements . . . . . . . . . . . . . . . . 71

4 High momentum electron background studies $\quad 81$

4.1 High momentum electron simulations . . . . . . . . . . . . . 82

4.1.1 Tracing particles with virtual detectors $\ldots \ldots \ldots \ldots$

4.1 .2 Resampling . . . . . . . . . . . . . . . . . 84

4.1 .3 Stage 4 simulation . . . . . . . . . . . . . . . . 87

4.2 Background estimation algorithm $\ldots \ldots \ldots \ldots \ldots$

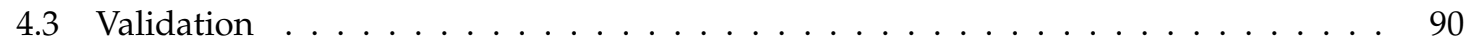

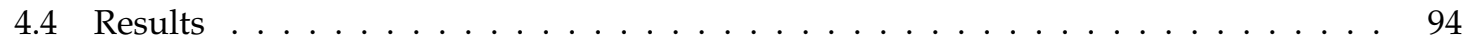

5 Tests of the Transport Solenoid field with low momentum electrons 97

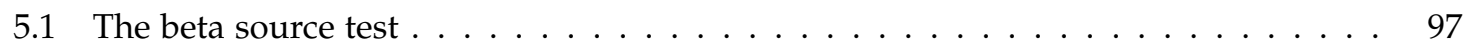

5.1 .1 Motivation . . . . . . . . . . . . . . . . . . . . . 97

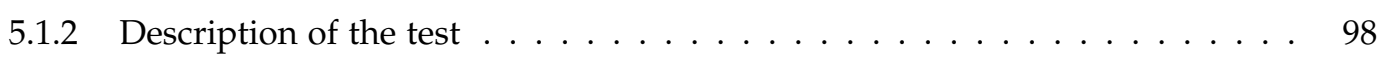

5.1 .3 Medium effects . . . . . . . . . . . . . . . . . 98

5.1 .4 Source model . . . . . . . . . . . . . . . . . . . . . . 100

5.1 .5 Detector model . . . . . . . . . . . . . . . . . . . . . . . . . . 102

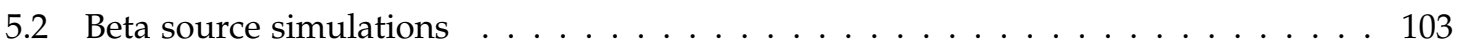

5.2 .1 Simulation parameters . . . . . . . . . . . . . . . . . . . 103

5.2 .2 Outline of the study . . . . . . . . . . . . . . . . . . . . . . 104

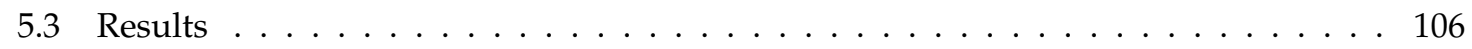

5.3.1 Study of the effect of outliers . . . . . . . . . . . . . . . 106

5.3.2 Study of the effect of detector resolution and secondary particles . . . . . . 107

5.3 .3 Rotations . . . . . . . . . . . . . . . . . . . . . . . . . . . . 112

5.3 .4 Parallel coil displacements . . . . . . . . . . . . . . . . . . 125

5.3.5 Dependence on vacuum conditions . . . . . . . . . . . . . . . 125

5.4 Nextsteps . . . . . . . . . . . . . . . . . . . . . . . . . . . . 130 
6 Conclusions

Bibliography

135 


\section{List OF Figures}

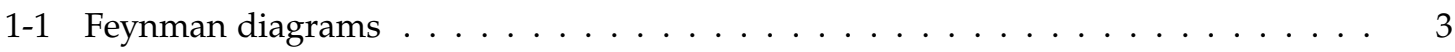

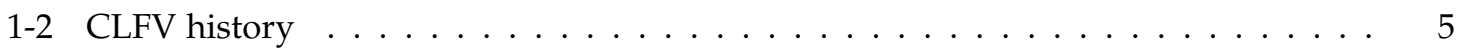

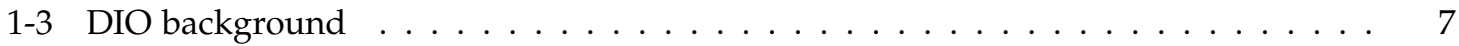

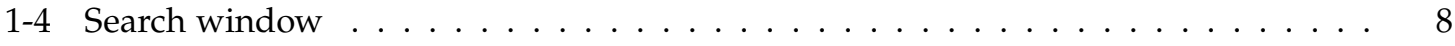

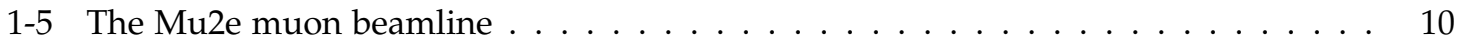

1-6 The Mu2e tracker . . . . . . . . . . . . . . . . . . . . . . . 11

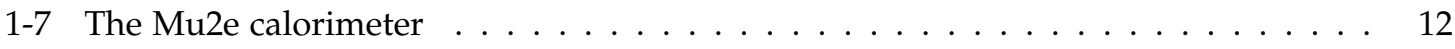

1-8 The Mu2e Cosmic Ray Veto . . . . . . . . . . . . . . . . . . . . . . 12

1-9 The Mu2e magnetic field . . . . . . . . . . . . . . . . . . . . . . 14

1-10 The Mu2e coordinate systems . . . . . . . . . . . . . . . . . 15

1-11 The Transport Solenoid . . . . . . . . . . . . . . . . . . . . . . . 18

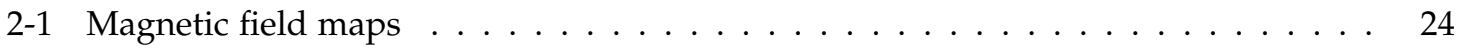

2-2 Mau9-GA00 field map comparisons in 3D . . . . . . . . . . . . . . 28

2-3 Mau9-GA00 field map comparisons . . . . . . . . . . . . . . . . . . . . . . 29

2-4 GA00-GA05 DS field differences $>1 \mathrm{G} \ldots \ldots \ldots \ldots \ldots$

2-5 GA05-GA00 field map comparisons in 3D . . . . . . . . . . . . . . 31

2-6 Interpolation test with random points in DS . . . . . . . . . . . . . . 32

2-7 Mau9-SolCalc field map comparisons in 3D . . . . . . . . . . . . . . . 36

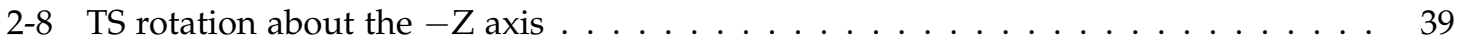

2-9 Comparisons between default and rotated Mau10 field maps . . . . . . . . . . . 39

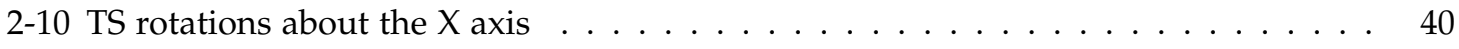

2-11 Comparisons between default and rotated SolCalc field maps . . . . . . . . . . . 40

2-12 Parallel coil displacements of TS coils about the $\mathrm{Y}$ axis . . . . . . . . . . . . 41

2-13 Comparisons between default and shifted SolCalc field maps . . . . . . . . . . . . 41

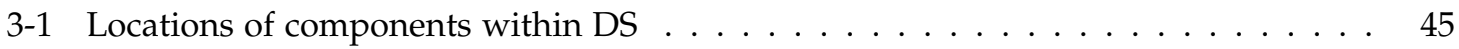

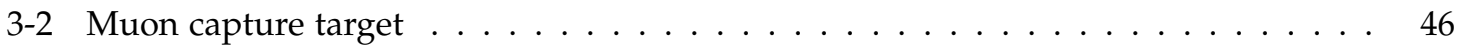


3-3 Polar distribution of captured muons . . . . . . . . . . . . . . . 47

3-4 Muon stopping rate for TS rotation of $0.15^{\circ}$ about $-\mathrm{Z} \ldots \ldots \ldots \ldots$

3-5 Muon stopping rate for TS rotation of $0.50^{\circ}$ about $-Z \ldots \ldots \ldots 2$

3-6 Pion stopping rate for TS rotation of $0.15^{\circ}$ about $-Z \ldots \ldots \ldots$

3-7 Pion stopping rate for TS rotation of $0.50^{\circ}$ about $-\mathrm{Z} \ldots \ldots \ldots \ldots$

3-8 Muon stopping rate for TSu rotation of $1^{\circ}$ about $+X$ and $1^{\circ}$ about $-X$ of TSd $\ldots 55$

3-9 Muon stopping rate for TSu rotation of $1^{\circ}$ about $-X$ and $1^{\circ}$ about $+X$ of TSd $\ldots 56$

3-10 Muon stopping rate for TSu rotation of $1^{\circ}$ about $+X$ and $1^{\circ}$ about $+X$ of TSd . . . 57

3-11 Muon stopping rate for TSu rotation of $1^{\circ}$ about $-X$ and $1^{\circ}$ about $-X$ of TSd . . . 58

3-12 Pion stopping rate for TSu rotation of $1^{\circ}$ about $+X$ and $1^{\circ}$ about $-X$ of TSd . . . 59

3-13 Pion stopping rate for TSu rotation of $1^{\circ}$ about $-X$ and $1^{\circ}$ about $+X$ of TSd $\ldots 60$

3-14 Pion stopping rate for TSu rotation of $1^{\circ}$ about $+X$ and $1^{\circ}$ about $+X$ of TSd $\ldots .61$

3-15 Pion stopping rate for TSu rotation of $1^{\circ}$ about $-X$ and $1^{\circ}$ about $-X$ of TSd . . . 62

3-16 Muon stopping rate for TSu rotation of $0.1^{\circ}$ about $+X$ and $0.1^{\circ}$ about $-X$ of TSd . 63

3-17 Muon stopping rate for TSu rotation of $0.1^{\circ}$ about $-X$ and $0.1^{\circ}$ about $+X$ of TSd . 64

3-18 Muon stopping rate for TSu rotation of $0.1^{\circ}$ about $+X$ and $0.1^{\circ}$ about $+X$ of TSd . 65

3-19 Muon stopping rate for TSu rotation of $0.1^{\circ}$ about $-X$ and $0.1^{\circ}$ about $-X$ of TSd . 66

3-20 Pion stopping rate for TSu rotation of $0.1^{\circ}$ about $+X$ and $0.1^{\circ}$ about $-X$ of TSd . . 67

3-21 Pion stopping rate for TSu rotation of $0.1^{\circ}$ about $-X$ and $0.1^{\circ}$ about $+X$ of TSd . . 68

3-22 Pion stopping rate for TSu rotation of $0.1^{\circ}$ about $+X$ and $0.1^{\circ}$ about $+X$ of TSd . $\quad 69$

3-23 Pion stopping rate for TSu rotation of $0.1^{\circ}$ about $-X$ and $0.1^{\circ}$ about $-X$ of TSd . . 70

3-24 Muon stopping rate for coil displacement about $+Y$ in TSu and $+Y$ in TSd $\ldots . .73$

3-25 Muon stopping rate for coil displacement about $-Y$ in TSu and $-Y$ in TSd . . . . 74

3-26 Muon stopping rate for coil displacement about $+Y$ in TSu and $-Y$ in TSd . . . . 75

3-27 Muon stopping rate for coil displacement about $-Y$ in TSu and $+Y$ in TSd . . . . 76

3-28 Pion stopping rate for coil displacement about $+Y$ in TSu and $+Y$ in TSd . . . . 77

3-29 Pion stopping rate for coil displacement about $-Y$ in TSu and $-Y$ in TSd . . . . . 78

3-30 Pion stopping rate for coil displacement about $+Y$ in TSu and $-Y$ in TSd . . . . 79

3-31 Pion stopping rate for coil displacement about $-Y$ in TSu and $+Y$ in TSd . . . . 80

4-1 Spatial and momentum distribution of beam electrons at the capture target . . . . 82

4-2 Time distribution of beam electrons at the capture target $\ldots \ldots \ldots \ldots$

4-3 Pitch distribution of beam electrons at the capture target . . . . . . . . . . 84

$4-4$ Resampling fit function . . . . . . . . . . . . . . . 85

4-5 Spatial and momentum distribution of electrons after resampling . . . . . . . . 86

4-6 Momentum distribution of all electrons hitting the tracker . . . . . . . . . . . . 87

4-7 Position distribution of all electrons hitting the tracker $\ldots \ldots \ldots$. . . . . 88 
4-8 $\quad p_{z} / p$ and $p_{T}$ distribution of all electrons hitting the tracker $\ldots \ldots \ldots . \ldots 8$

4-9 Position distribution of the signal-like electrons hitting the tracker . . . . . . . . . 89

$4-10$ Locations of electron creation up to VD6 . . . . . . . . . . . . . . . . . 90

4-11 Locations of electron creation along the transport line . . . . . . . . . . . . . . 91

4-12 Electron location at the beginning of the capture target . . . . . . . . . . . . . 92

4-13 Electron hits at the tracker . . . . . . . . . . . . . . . . . . . . . 93

4-14 Electron trajectories in Mu2e magnetic field . . . . . . . . . . . . . . . . . . . . . . 94

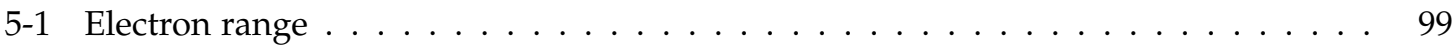

5-2 $\quad \beta^{-}$decay of the ${ }^{90} \mathrm{Sr}$ into ${ }^{90} \mathrm{Y}$, which decays into an electron and ${ }^{90} \mathrm{Zr} . \quad \ldots \ldots$. . . . . 101

5-3 Momentum spectra of emission probabilities . . . . . . . . . . . . . . . . . 102

5-4 Plan view of the Mu2e solenoids and field maps . . . . . . . . . . . . . . . 103

$5-5 \quad \beta$ source locations . . . . . . . . . . . . . . . . . . . . . . . . . . . . . 104

5-6 Electron beam profiles with a misaligned field . . . . . . . . . . . . . . . 105

5-7 Study of the effect of detector resolution and $\delta$ rays at VD3 $\ldots \ldots \ldots \ldots$

5-8 Study of the effect of detector resolution and $\delta$ rays at VD8 $\ldots \ldots \ldots \ldots \ldots$

$5-9$ Electron spots for Mau10 default maps . . . . . . . . . . . . . . . . . . 113

5-10 Momentum spectrum for Mau10 nominal maps . . . . . . . . . . . . . . . . . . 114

5-11 Results for TS rotation about $-\mathrm{Z}$ axis at VD3 . . . . . . . . . . . . . . . . . 115

$5-12$ Results for TS rotation about $-\mathrm{Z}$ axis at VD8 $\ldots \ldots \ldots \ldots \ldots \ldots$

5-13 Results for TS rotation about $-\mathrm{Z}$ axis below the $\mathrm{XZ}$ plane at VD3 . . . . . . . . 117

5-14 Results for TS rotation about $-\mathrm{Z}$ axis below the XZ plane at VD8 . . . . . . . . 118

5-15 Results for TS rotation about $-Z$ axis in small steps above the XZ plane at VD3 . . 119

5-16 Results for TS rotation about $-Z$ axis in small steps above the $X Z$ plane at VD8 . . 120

5-17 Results for $1^{\circ}$ TS rotation at VD3 . . . . . . . . . . . . . . . . . . . . . . 121

$5-18$ Results for $1^{\circ}$ TS rotation at VD8 . . . . . . . . . . . . . . . . . . . . . 122

5-19 Results for $0.1^{\circ}$ TS rotation at VD3 . . . . . . . . . . . . . . . . . . . . 123

5-20 Results for $0.1^{\circ}$ TS rotation at VD8 . . . . . . . . . . . . . . . . . . . . . . . 124

5-21 Results for TS parallel coil displacements at VD3 $\ldots \ldots \ldots \ldots \ldots \ldots$

5-22 Results for TS parallel coil displacements at VD8 . . . . . . . . . . . . . . 127

$5-23$ Vacuum study at VD3 . . . . . . . . . . . . . . . . . . . . 128

$5-24$ Vacuum study at VD8 . . . . . . . . . . . . . . . . . . . . . . . . . . 129 


\section{LIST OF TABLES}

1.1 Estimated backgrounds yields . . . . . . . . . . . . . . . 9

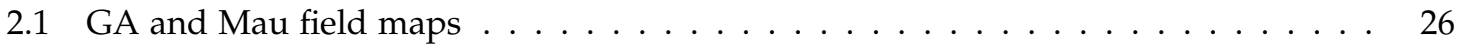

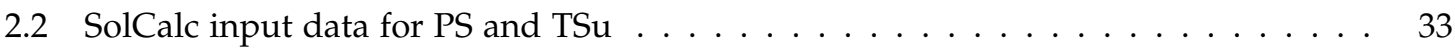

2.3 SolCalc input data for TSd and DS . . . . . . . . . . . . . 34

2.4 TS rotations . . . . . . . . . . . . . . . . . . . . . . . . . . . . . . 37

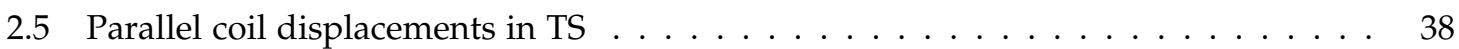

3.1 Proton beam parameters for the simulation of stopping rates $\ldots \ldots \ldots$. . . . . 47

3.2 Muon stopping rates for rotated maps . . . . . . . . . . . . . . . . . 49

3.3 Pion stopping rates for rotated maps . . . . . . . . . . . . . . . . . . 49

3.4 Fractional yield differences for $\mu^{-}$and $\pi^{-}$for rotated maps . . . . . . . . . 50

3.5 Muon stopping rates for parallel coil displacements . . . . . . . . . . . . . . . . 71

3.6 Pion Stopping Rates for parallel coil displacements _ . . . . . . . . . . . . . . 71

3.7 Fractional yield differences for $\mu^{-}$and $\pi^{-}$for parallel coil displacements $\ldots \ldots .72$

4.1 Resample fitting uncertainties . . . . . . . . . . . . . . . 85

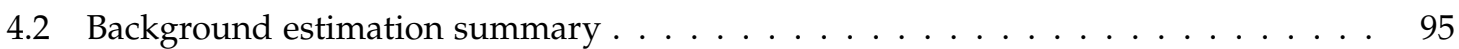

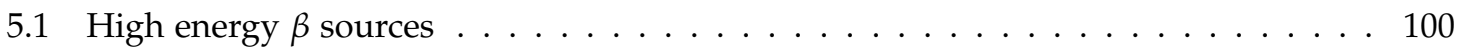

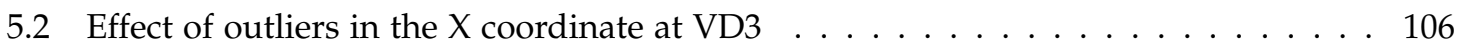

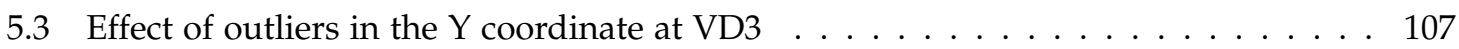

5.4 Effect of outliers in the $\mathrm{X}$ coordinate at VD8 $\ldots \ldots \ldots \ldots \ldots \ldots$

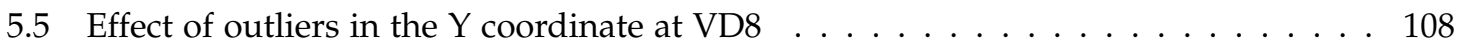

5.6 Effect of detector resolution and $\delta$ rays $\ldots \ldots \ldots \ldots$

5.7 Effect of detector resolution and $\delta$ rays $\ldots \ldots \ldots \ldots \ldots$

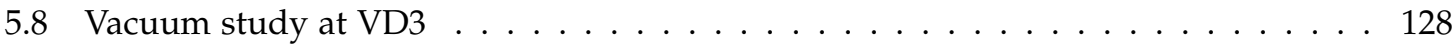

5.9 Vacuum study at VD8 . . . . . . . . . . . . . . . . . . . . . . . . . . . . . . . . . . . . 129 


\section{CHAPTER 1}

\section{INTRODUCTION}

\subsection{THE Mu2E EXPERIMENT}

The Mu2e experiment at Fermilab proposes to measure the ratio of the rate of the neutrinoless coherent conversion of muons into electrons in the field of a nucleus, to the rate of muon capture on the nucleus:

$$
R_{\mu e}=\frac{\mu^{-}+A(Z, N) \rightarrow e^{-}+A(Z, N)}{\mu^{-}+A(Z, N) \rightarrow v_{\mu}+A(Z-1, N)}
$$

This quantity will be measured by the formation of the most intense muon beam in the World, with a flux of $\sim 10^{10} \mu^{-}$/s producing a total of $\sim 10^{18}$ muonic $\mathrm{Al}$ atoms. Depending on the momentum and on the impact parameter of the incident muons with respect to the nucleus, the muon has a probability to be captured in different atomic shells. When muons are captured in higher shells, the muonic atom is in an exited state whose life time is $\leq 1$ ps, i.e. much shorter than the life time of the ground state (864 ns). Muons then cascade to lower shells emitting X-rays. By using the ratio of Equation 1.1, common experimental and theoretical uncertainties in the numerator and denominator cancel out.

When the captured muon is in a 1s state, the muon and Al nucleus wave functions overlap, so that the muonic atoms are unstable and decay via three possible processes:

1. Nuclear capture $(\sim 61 \%): \mu^{-}+{ }_{13}^{27} \mathrm{Al} \rightarrow v_{\mu}+{ }_{12}^{27} \mathrm{Mg}^{*}$;

2. Muon decay in orbit $(\sim 39 \%): \mu^{-}+{ }_{13}^{27} \mathrm{Al} \rightarrow e^{-}+\bar{v}_{e}+v_{\mu}+{ }_{13}^{27} \mathrm{Al}$;

3. Muon to electron conversion $\left(<10^{-12}\right): \mu^{-}+{ }_{13}^{27} \mathrm{Al} \rightarrow e^{-}+{ }_{13}^{27} \mathrm{Al}$.

The conversion process is the signal of the experiment, with a distinct signature of a single electron carrying an energy of $E_{e}=m_{\mu} c^{2}-E_{\text {bind }}-E_{\text {recoil }}=104.96 \mathrm{MeV}$, where $E_{\text {bind }}$ is the binding energy of the muon with the $\mathrm{Al}$ nucleus and $E_{\text {recoil }}$ is the kinetic energy of the recoiling $\mathrm{Al}$ nucleus. Muon 
decay in orbit (DIO) is the dominant background of the experiment, producing electrons of energy up to $104.96 \mathrm{MeV}$. Nuclear capture serves as a normalization (or "effective luminosity") factor for the signal, since it can be both calculated using the amplitude of muon capture in the electromagnetic field of an $\mathrm{Al}$ nucleus and measured by detecting the photons coming from cascade de-excitations of the excited daughter nuclei $\mathrm{Mg}^{*}$.

Mu2e intends to probe four orders of magnitude beyond the current best experimental limit, measuring $R_{\mu e}$ with a single-event sensitivity of $2.87 \times 10^{-17}$. The conversion process is an example of charged lepton flavor violation (CLFV), a process that has never been observed experimentally. Observation of this process would provide unambiguous evidence for physics beyond the Standard Model (SM). It can help illuminate discoveries made at the LHC, by observing for example signatures of the new physics in complementary channels, or point to new physics at scales beyond the reach of the LHC.

\subsubsection{Motivation}

In the Standard Model (SM) the assumption of massless neutrinos excludes lepton flavor violation. However, the observation from various experiments of neutrino oscillations has shown that lepton flavor is not a conserved quantum number, since neutrinos produced with a given flavor change it as they propagate. On the contrary, in the quark sector, the $\mathrm{SU}(2)$-hypercharge symmetry breaking due to the weak interaction leads from gauge (massless) quarks to physical (massive) quarks through a unitary transformation, introducing a weak mixing between the physical quark flavor states [Paschos(2007)].

Lepton flavor violation (LFV) processes involving charged leptons have never been observed, although, in principle, there is no fundamental symmetry that forbids processes like $\mu \rightarrow e \gamma$, $\tau \rightarrow e \mu \mu$ or $K_{L} \rightarrow e \mu$. This fact can be well understood in some minimal frameworks, such as the minimal extension of the SM with Dirac neutrinos. In this model that includes finite neutrino mass terms in the Lagrangian, charge lepton flavor violation (CLFV) is predicted, but transitions are suppressed by sums over $\left(\Delta m_{i j}^{2} / M_{W}^{2}\right)^{2}$, where $\Delta m_{i j}^{2}$ is the mass-squared difference between the $i^{t h}$ and $j^{\text {th }}$ neutrino mass eigenstates, and $M_{W}$ is the $W$ boson mass [Marciano et al.(2008)]. Because the neutrino mass difference is very small $\left(\Delta m_{i j}^{2} \leq 10^{-5} \mathrm{eV}^{2}\right.$ [Beringer et al.(2012)]) with respect to the $W$ boson mass, the rates of a CLFV decays in this extended version of the SM are effectively zero (e.g. $<10^{-50}$ for both $\mu^{+} \rightarrow e^{+} \gamma$ and $\mu^{-} N \rightarrow e^{-} N$ [Beringer et al.(2012), Marciano et al.(2008)]). Thus, an observation of CLFV process would represent a clear evidence of new physics.

There are various new physics models which predict rates for CLFV processes. These include the Minimal Supersymmetric Standard Model (MSSM) with right-handed neutrinos, SUSY with R-parity violation, as well as models with leptoquarks, new gauge bosons, large extra-dimensions, or a non-minimal Higgs sector [Raidal et al.(2008)]. In all these models, lepton flavor mixing is 
made possible either by changing the group structure of the SM, introducing new symmetries and new interactions, or by extending the dimension of matter fields, introducing new fermion families.

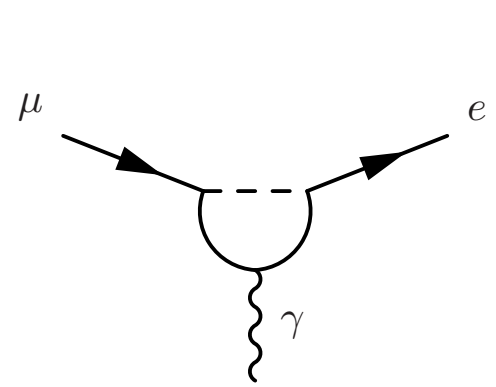

(a)

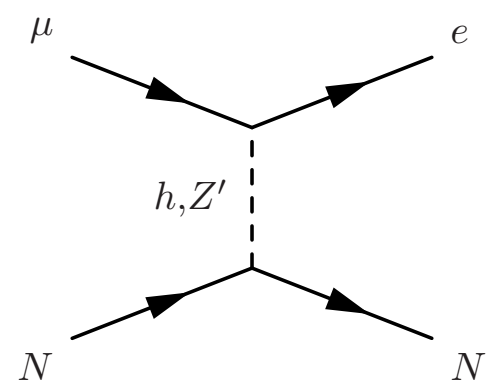

(c)

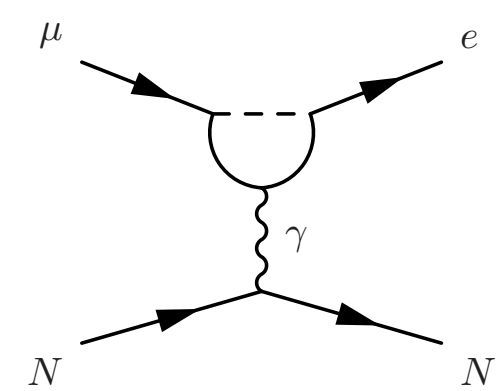

(b)

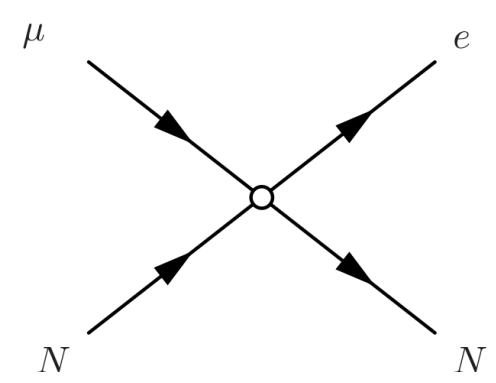

(d)

Figure 1-1: Class of diagrams that can contribute to $\mu^{ \pm} \rightarrow e^{ \pm}+\gamma$. The upper two diagrams describe a dipole interaction in the free space (1-1(a)) or in the field of a nucleus (1-1(b)). The lower right diagram (1-1(d)) is an effective parametrization of new physics with a "contact" interaction in place of the loop and the photon exchange of diagram 1-1(b) or the heavy boson internal line of diagram 1-1(c).

The Mu2e experiment, with a single-event sensitivity of a few $10^{-17}$ for the ratio of $\mu^{-} N \rightarrow e^{-} N$ conversions to muon capture, has real discovery potential over a wide range of new physics models and may prove to help to discriminate among models. Two classes of diagrams can contribute to the muon to electron conversion, as shown in Figure 1-1. The first class of "tree" diagrams (1-1(c) and 1-1(d)) describes direct $\mu \rightarrow e$ conversion. The second class of dipole interaction or "penguin" diagrams (1-1(a) and 1-1(b)) describes the radiative $\mu \rightarrow e+\gamma$ decay. 1-1(a) describes conversion in the free space. Diagram 1-1(b) describes the conversion in the field of a nucleus, which can occur via a virtual photon or a heavy neutral boson. Since the exchanged virtual particle can be a photon or a heavy neutral boson, $\mu N \rightarrow e N$ conversion has access to a broader range of new physics than $\mu^{ \pm} \rightarrow e^{ \pm} e^{+} e^{-}$and $\mu \rightarrow e \gamma$, by examining the dependence of a possible signal on various muon capture targets. Diagrams 1-1(a) and 1-1(b) can proceed with many different sorts of particles in the loop, including, but not limited to, SUSY particles, heavy neutrinos and a second Higgs Doublet. If the broken line in those two diagrams is a SM neutrino (and then the solid line of the loop is a SM W boson), then the conversion proceeds through the oscillation of the broken line, which suppresses the contribution of the diagram by many orders of magnitude. If the loop involves new particles, both those diagrams describe new physics. Diagram 1-1(d) is a description of new 
physics leading to neutrinoless conversion, with an effective "contact" interaction of infinite mass scale. The contact interaction is parametrized by a scale-dependent coupling parameter and is thus non-renormalizable.

\subsubsection{Present status of CLFV searches using muons}

In general, CLFV can be studied via a large variety of processes ([Marciano et al.(2008)]):

- muon decay, such as $\mu \rightarrow e \gamma, \mu^{ \pm} \rightarrow e^{ \pm} e^{-} e^{+}$and muon coherent conversion;

- $\tau$ decays, such as $\tau \rightarrow \mu \gamma, \tau^{ \pm} \rightarrow \mu^{ \pm} \mu^{+} \mu^{-}$;

- meson decays: $\pi^{0} \rightarrow \mu e, K_{L}^{0} \rightarrow \mu e, K^{+} \rightarrow \pi^{+} \mu^{+} e^{-}$, etc;

- $Z^{0}$ decays, such as $Z^{0} \rightarrow \mu e$, etc;

- Higgs boson decay, such as $H \rightarrow \tau \mu$.

Because muons are abundantly produced at high-intensity proton accelerators and because their simple final states can be very precisely measured, the current best experimental bounds on LFV are obtained on processes of muon decays $\left(\mu^{+} \rightarrow e^{+} \gamma, \mu^{+} \rightarrow e^{+} e^{-} e^{+}\right)$and muon coherent conversion $\left(\mu^{-} N \rightarrow e^{-} N\right)$.

The current experimental limits (all at 90\% CL) on the branching ratios are: $\operatorname{BR}\left(\mu^{+} \rightarrow e^{+} \gamma\right.$ ) $<4.2 \times 10^{-13}$ [Baldini et al.(2016)], $\mathrm{BR}\left(\mu^{+} \rightarrow e^{+} e^{-} e^{+}\right)<1.0 \times 10^{-12}$ [Bellgardt et al.(1988)], and $\mathrm{R}_{\mu e}(\mathrm{Au})(\mu \rightarrow e)<7 \times 10^{-13}$ [Bertl et al.(2006)]. Figure 1-2 shows the history of the limits on the branching ratio of muon decays and muon conversion and the goals of the upcoming experiments [Bernstein and Cooper(2013)]. Searches in $\mu^{+} \rightarrow e^{+} \gamma, \mu^{ \pm} \rightarrow e^{ \pm} e^{-} e^{+}$, and $\mu N \rightarrow e N$ are complementary in that their sensitivity to CLFV is different depending on the underlying new physics [de Gouvea and Vogel(2013)]. In fact, if a signal of charged lepton flavor violation is observed, then the relative rates of $\mu^{+} \rightarrow e^{+} \gamma, \mu^{+} \rightarrow e^{+} e^{-} e^{+}$, and $\mu N \rightarrow e N$ can constrain the underlying physics responsible for the observed CLFV interactions.

In what concerns the radiative decay $\mu^{+} \rightarrow e^{+} \gamma$, the experiment leading to the most stringent constraints is MEG. Experimentally, a $\mu^{+} \rightarrow e^{+} \gamma$ event is characterized by a simple two-body final state: the electron and photon are emitted back to back in the rest frame of the decaying muon, with each carrying away an energy equal to half the muon mass (52.8 MeV), neglecting the tiny electron mass. Low-energy muons are stopped in a solid (known as a stopping target); in order to avoid formation of muonic atoms that would destroy the two-body kinematic signature, only positive muons are used. The largest background in this search is an accidental coincidence of a positron from the standard Michel decay of muons, $\mu \rightarrow e v \bar{v}$ and a relatively high energy photon from radiative muon decay, $\mu \rightarrow e v \bar{\nu} \gamma$. The current best limit has been obtained by the MEG experiment 


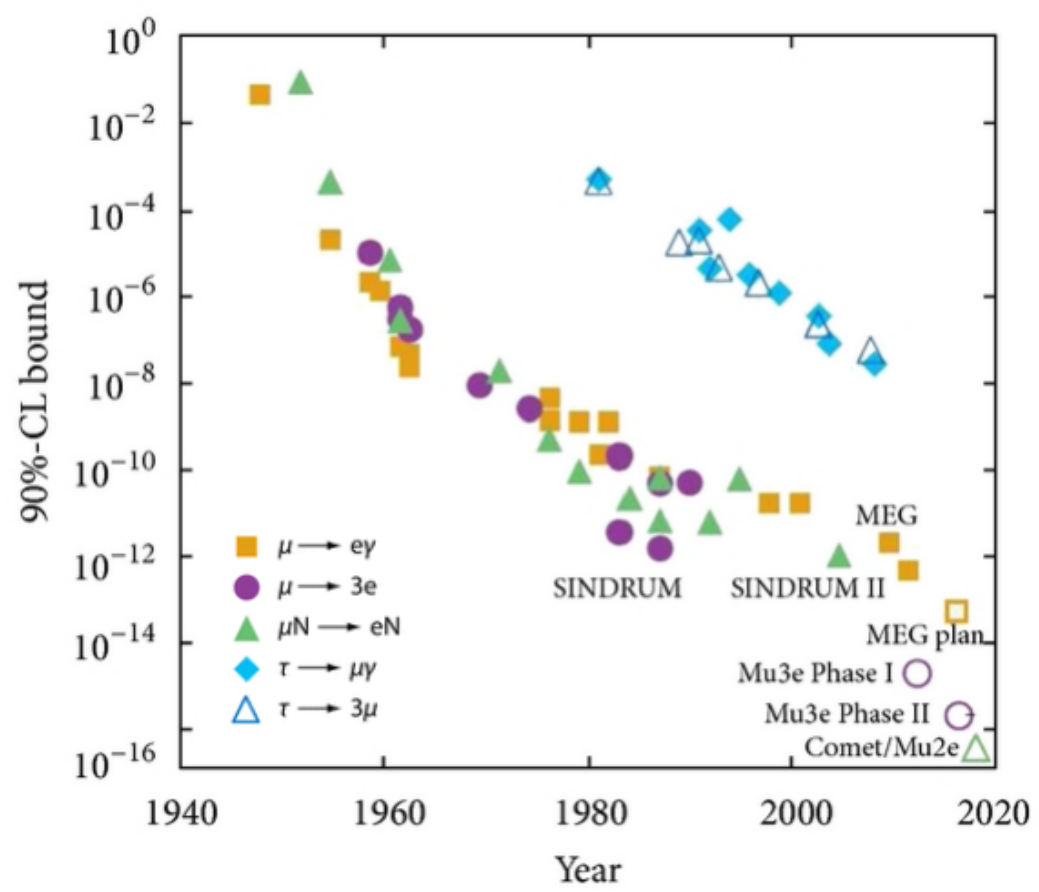

Figure 1-2: Experimental limits @ 90\% C.L. for branching fractions of CLFV processes involving muons and tau's as a function of time. The open symbols represent the expected sensitivities of future projects: MEG upgrade, Mu3e, Mu2e and COMET [Cei and Nicolò(2014)].

in 2016. An upgrade of the detector system is underway, in order to improve the sensitivity by another order of magnitude [Baldini et al.(2013)].

The present upper limit on $\mu^{+} \rightarrow e^{+} e^{+} e^{-}$branching ratio was obtained by the SINDRUM experiment at PSI in 1988. It used $\sim 5 \times 10^{6}$ positive muon beam, stopped in a double-cone shaped target. Muon decay products were detected by a magnetic spectrometer, made by five Multi Wire Proportional Chambers and a cylindrical array of 64 scintillators, arranged in a $0.33 \mathrm{~T}$ solenoid magnetic field.

The search for the $\mu \rightarrow e$ conversion has been carried out by the SINDRUM II experiment, performed at PSI in 1993: it focused negative muons with a momentum of $88 \mathrm{MeV} / \mathrm{c}$ and an intensity of $1.2 \times 10^{7} \mu^{-} / s$ on a Titanium target [Dohmen et al.(1993)]. During a 25 day run a total of $4.9 \times 10^{12}$ muons were stopped. The electron energy was measured with a spectrometer inside a superconducting solenoid with a 1 Tesla field. The spectrometer consisted of several cylindrical detectors surrounding the target on the beam axis. Two drift chambers provided the tracking while scintillation and Cerenkov hodoscopes were used for the timing of the track elements and electron identification. A scintillation beam counter in front of the target helped to recognize prompt background electrons produced by radiative capture of beam pions or beam electrons scattering off the target. The pion contamination was reduced by a factor of $10^{6}$ by passing the beam through a thin moderator that reduced the muon flux by $30 \%$. The few surviving pions had 
very low momenta and a simulation showed that $\sim 99.9 \%$ of them decayed before reaching the target. Electrons from radiative pion capture in the moderator could reach the target and scatter into the detector solid angle. This background was easily recognized since it was strongly peaked in the forward direction and had a characteristic time correlation with the cyclotron RF. The electron spectrum agreed well with the predictions for muon decay-in-orbit. No events were observed with energies consistent with the $\mu^{-}+N \rightarrow e^{-}+N$ hypothesis resulting in a limit of $4.3 \times 10^{-12}(90 \%$ CL).

In 2000 SINDRUM II performed a new search for muon to electron conversion using a 53 $\mathrm{MeV} / \mathrm{c}$ muon beam and a gold target [Bertl et al.(2006)]. The conversion energy for gold is 95.6 $\mathrm{MeV}$. During a 75 -day live time $4.4 \times 10^{13}$ muons were stopped. After removing forward prompt events, the electron spectrum was well described by muon decays in orbit and no events were observed in the signal region. One electron event, thought to be pion induced, was identified at higher energy. A final limit on muon to electron conversion in gold was set at $7 \times 10^{-13}$ (90\% CL).

\subsubsection{Mu2e signal and backgrounds}

Previous muon to electron conversion experiments have not observed events in the signal region. Mu2e proposes to improve on previous measurements by a factor of approximately 10,000, setting an upper limit of $R_{\mu e} \leq 6 \times 10^{-17}$ at the $90 \%$ confidence level.

In the coherent $\mu^{-} \rightarrow e^{-}$conversion in the field of a nucleus, the stopped muons are quickly captured by atoms $\left(10^{-10} \mathrm{~s}\right)$ and cascade down to $1 \mathrm{~S}$ orbitals. There, they can undergo decay with a rate of $\sim 5 \times 10^{5} \mathrm{~s}^{-1}$ (decay in orbit or DIO), weak capture $\mu^{-} p \rightarrow v_{\mu} n$ or coherent flavor changing conversion, $\mu^{-} N \rightarrow e^{-} N$. The last of these reactions is a powerful channel to search for CLFV, because it is characterized by a distinctive signal consisting in a mono-energetic electron with energy $E_{C E}$ :

$$
E_{C E}=m_{\mu}-B_{\mu}(Z, A)-R(A)
$$

where $m_{\mu}$ is the muon mass, $B_{\mu}(Z, A) \simeq \frac{Z^{2} \alpha^{2} m_{\mu}}{2}$ is the muonic atom binding energy and $R(A) \simeq \frac{m_{\mu}^{2}}{m_{N}}$ is the nuclear recoil energy for a nucleus with atomic number $\mathrm{Z}$ and mass number $\mathrm{A}$. In case of $\mathrm{Al}$, which is the major candidate for Mu2e experiment, $E_{c e}=104.96 \mathrm{MeV}$. The coherent conversion leaves the nucleus intact, and there is only one detectable particle in the final state.

At the proposed Mu2e sensitivity there are a number of processes that can mimic a $\mu^{-}+N \rightarrow$ $e^{-}+N$ signal. Controlling these potential backgrounds drives the overall design of Mu2e. These backgrounds result principally from five sources:

1. intrinsic processes that scale with beam intensity; these include muon decay-in-orbit (DIO) and radiative muon capture (RMC);

2. processes that are delayed because of particles that spiral slowly down the muon beam line, 
such as antiprotons;

3. prompt processes where the detected electron is nearly coincident in time with the arrival of a beam particle at the muon stopping target (e.g. radiative pion capture, RPC);

4. electrons or muons that are initiated by cosmic rays;

5. events that result from reconstruction errors induced by additional activity in the detector from conventional processes.

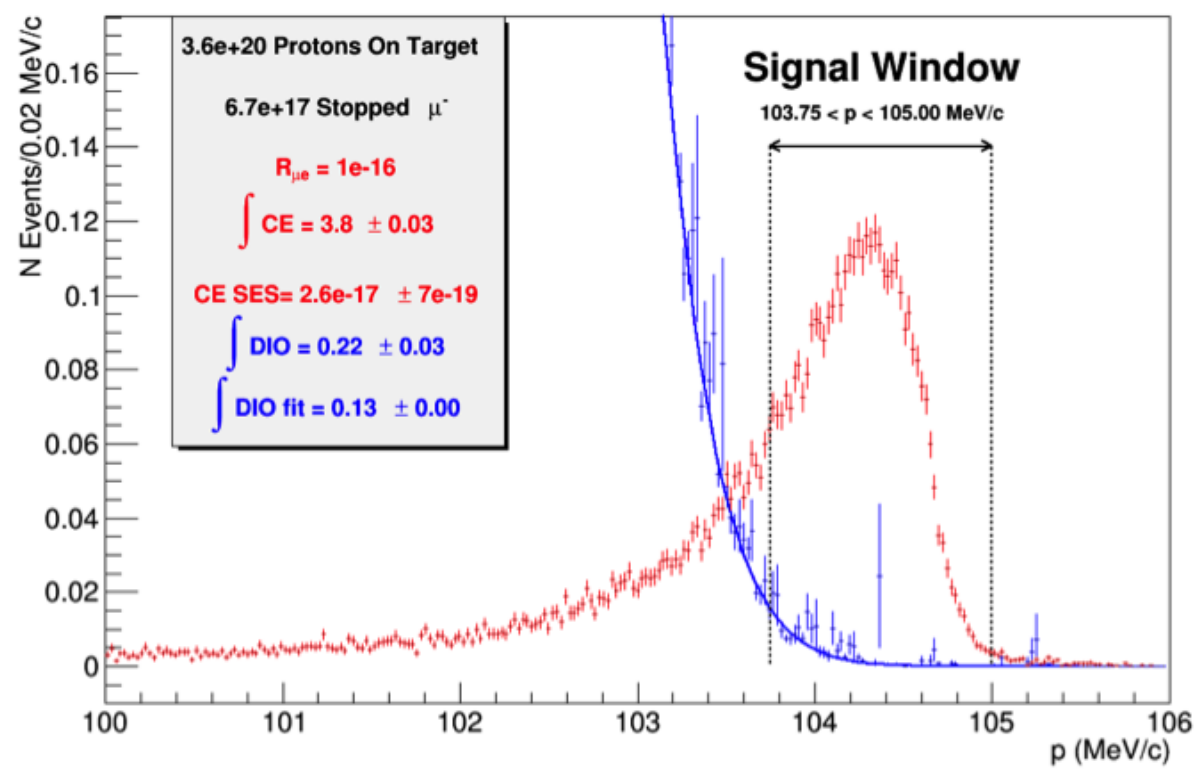

Figure 1-3: The simulated-reconstructed momentum spectrum for DIO events (blue) and conversion electron (CE) events surviving the track selection criteria and assuming $R_{\mu e}=10^{-16}$. The distributions are each normalized to the total number of muon stops expected for $3.6 \times 10^{20}$ protons on target.

DIO produces electrons with a continuous energy spectrum, as shown in blue in Figure 1-3; the shape is a distorted Michel spectrum with a long tail, due to radiative corrections in which the outgoing electron coherently exchanges photons with the nucleus or emits real photons which escape detection. In one extreme configuration, both neutrinos are at rest and the electron recoils against the intact $\mathrm{Al}$ nucleus. This is the configuration in which the electron has the maximum energy in the lab frame, about $105 \mathrm{MeV}$ for muonic Al. This energy is equal to the muon mass, less a small correction for the K-shell binding energy and an even smaller correction for nuclear recoil. The $\mu$ to $e$ conversion process produces a mono-energetic electron with an energy equal to that of the endpoint of the continuous spectrum from DIO. An intrinsic background comes from electrons in the high energy tail of the DIO spectrum that are mis-measured with a momentum in the signal region. Because of the rapid decrease in the DIO rate as the electron energy approaches the end point (approximately as $\left.\left(E_{\text {endpoint }}-E_{e}\right)^{5}\right)$, the background can be suppressed through adequate 
resolution on the electron momentum. To reduce the DIO background, the energy resolution must be narrow and high energy tails must be suppressed. This depends on the intrinsic resolution of the tracker detector as well as on the amount of material traversed by conversion electrons (CE).

Muons can also undergo radiative muonic capture (RMC) on the nucleus ( $\mu^{-} A l \rightarrow \gamma v_{\mu} M g$ ). The photon can be emitted internally or externally and convert to electron-positron pair in the stopping target or other surrounding material, producing an electron near the conversion electron energy.

The beam protons of $8 \mathrm{GeV}$ kinetic energy are above the production energy threshold for antiprotons. These particles do not decay and carry a negative electric charge, thus they can propagate through the Transport Solenoid and reach the stopping target. Antiprotons with momenta less than $100 \mathrm{MeV} / \mathrm{c}$ travel slowly, with speed less than $0.1 \mathrm{c}$; they spiral slowly through the Transport Solenoid and can take up to several micro-seconds to reach the Detector Solenoid. Consequently the expected flux of antiprotons at the stopping target is nearly constant in time so that the delayed live gate and the extinction system do not effectively mitigate the resulting background. They can then annihilate with protons in the material of the DS environment, producing events of nearly 2 $\mathrm{GeV}$ total energy, which can give a large variety of secondary particles and eventually signal-like electrons in the detectors.

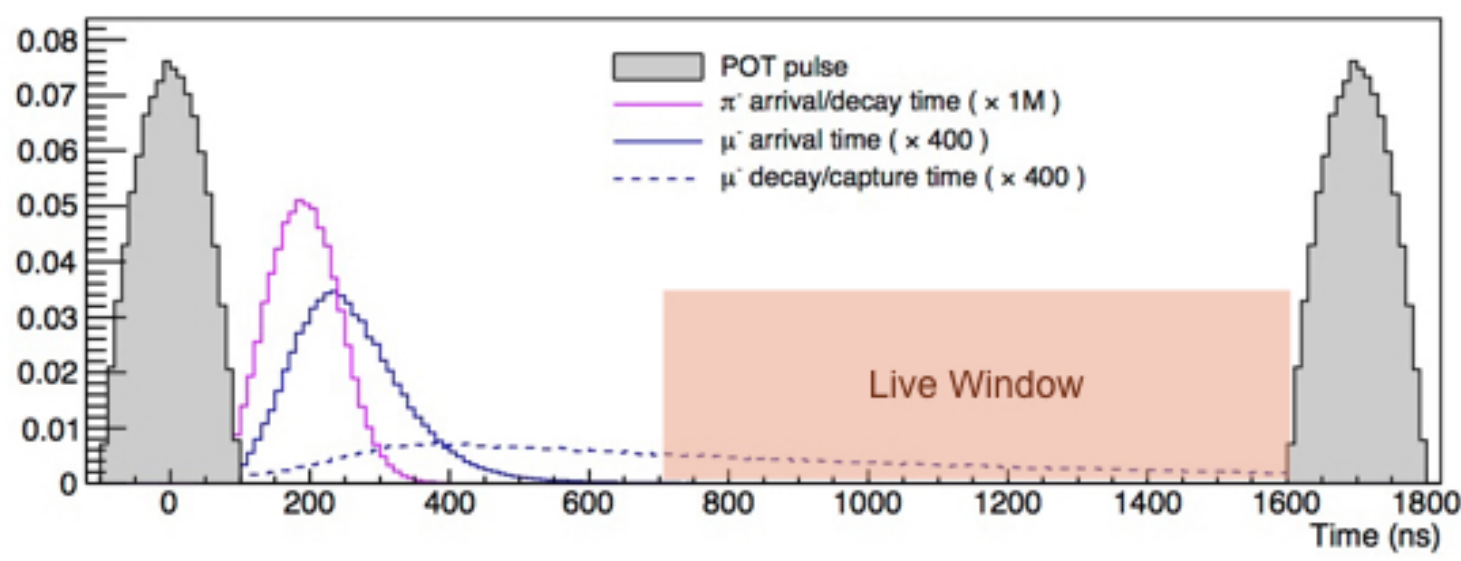

Figure 1-4: The Mu2e spill cycle for the proton beam and the delayed search window that allows for the effective elimination of prompt backgrounds when the number of protons between the pulses is suppressed to the required level. The width of proton on target (POT) pulse is of $200 \mathrm{~ns}$, while the prompt flash lasts $700 \mathrm{~ns}$. The start time and duration of the search window (995 ns) shown in the figure is illustrative. The exact delay and duration are optimized to keep pion-capture backgrounds at a low level.

The $\mu^{-}$beam that reaches the stopping target is contaminated by many $e^{-}$and some $\pi^{-}$, both of which can produce false signals when they interact with the stopping targets. Pions can produce background when they are absorbed in the stopping target or surrounding material and produce a 
high energy photon through radiative pion capture (RPC):

$$
\pi^{-}+(A, Z) \rightarrow \gamma+(A, Z-1)^{*}
$$

The outgoing photon can again convert to electron-positron pair, producing an electron that can mimic the signal. To defeat RPC and other prompt backgrounds, the experiment exploits the lifetime of muonic $\mathrm{Al}$, about 864 ns: as illustrated in Figure 1-4, Mu2e waits for 700 ns following the arrival of the proton bunch at the production target and then begins counting electrons that are emitted from the foils of the stopping target. By this time, all of the beam from the production target has passed through the stopping target and the prompt backgrounds have died away. After a total of $1695 \mathrm{~ns}$ the cycle is repeated.

It is also critical that few protons arrive at the production target between the bunches. If protons arrive out of time, they can produce $e^{-}$and $\pi^{-}$that arrive at the stopping target within the live gate. To reduce this background Mu2e requires an extinction factor of $10^{-10}$; that is, for every $10^{10}$ protons arriving at the production target within the bunch, there should be no more than 1 proton arriving between bunches.

Signal-like events can also be induced by cosmic rays (mostly muons, producing photons and electrons through bremsstrahlung). If cosmic rays have trajectories that appear to originate at the stopping target, they can fake a muon conversion electron. In order to identify and reject incoming cosmic rays, shielding and veto counters around the Detector Solenoid and particle identification are needed.

Table 1.1: A summary of the estimated background yields. The total run time @ $2 \times 10^{7}$ s/yr is of 3 years, with a corresponding number of protons on target of $1.2 \times 10^{20}$. An extinction of $10^{-10}$ and a cosmic ray veto inefficiency of $10^{-4}$ are used. Intrinsic backgrounds are those that scale with the number of stopped muons, Late Arriving backgrounds are those with a strong dependence on the achieved extinction, and Miscellaneous backgrounds are those that don't fall into the previous two categories.

\begin{tabular}{r|lr}
\hline \hline CAtegory & BACKGROund PROCESS & Estimated Yield (EVENTS) \\
\hline \hline \multirow{2}{*}{ Intrinsic } & Muon decay-in-orbit (DIO) & $0.199 \pm 0.092$ \\
& Muon capture (RMC) & $0.000_{-0.000}^{+0.004}$ \\
& Pion Capture (RPC) & $0.023 \pm 0.006$ \\
Late Arriving & Muon decay-in-flight $(\mu$-DIF) & $<0.003$ \\
& Pion decay-in-flight $(\pi$-DIF) & $0.001 \pm<0.001$ \\
& Beam electrons & $0.003 \pm 0.001$ \\
Miscellaneous & Antiproton induced & $0.047 \pm 0.024$ \\
& Cosmic ray induced & $0.082 \pm 0.018$ \\
\hline \hline
\end{tabular}


The dominant sources of false signal electrons are expected to be mis-measured DIO electrons $\left(0.199 \pm 0.092\right.$ events), radiative $\pi^{-}$capture on the target foils ( $0.023 \pm 0.006$ events $), \mu$ decay in flight $(<0.003$ events), antiproton $(0.047 \pm 0.024$ events $)$ and cosmic ray induced $(0.082 \pm 0.020$ events). These and three other processes add to total estimated background of $0.36 \pm 0.10$ events (Table 1.1). These numbers are quoted for $1.2 \times 10^{20}$ protons on target.

\subsubsection{Mu2e instrumentation}

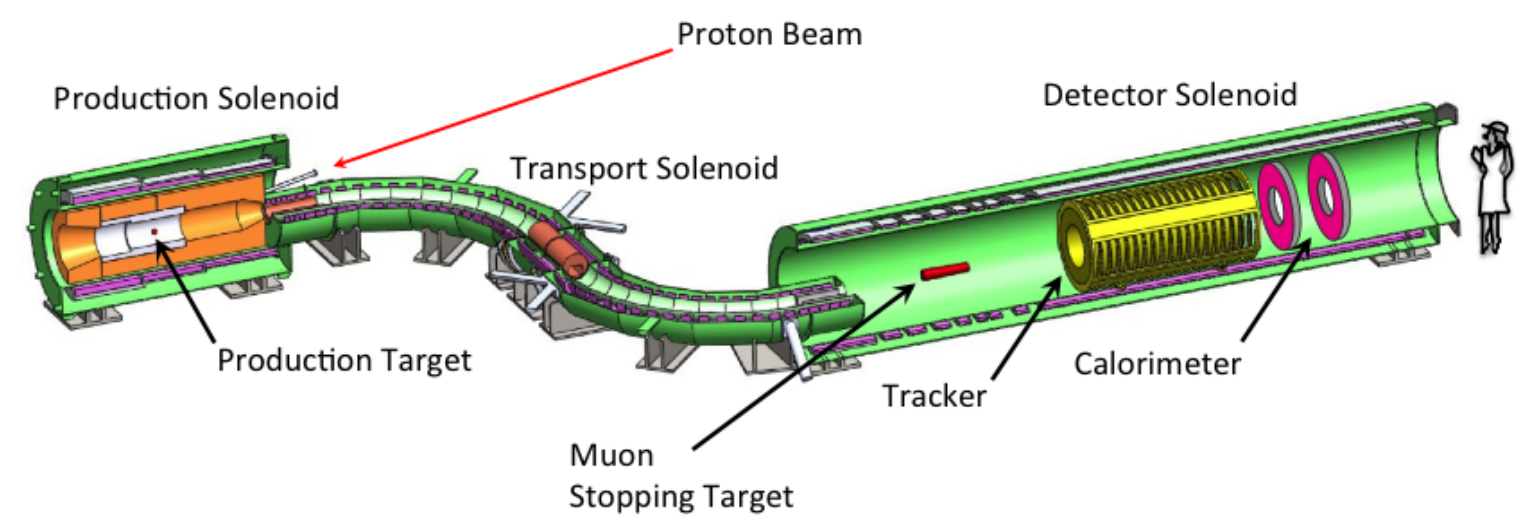

Figure 1-5: The Mu2e muon beamline showing the positions of the primary proton beam, the three solenoids, the production target, the stopping targets, and the detectors.

The Mu2e experimental apparatus (see Figure 1-5) consists of an integrated array of superconducting solenoids, which forms a graded magnetic system that includes the Production Solenoid (PS), the Transport Solenoid (TS) and the Detector Solenoid (DS).

The muon beam is produced using $8 \mathrm{GeV}$ protons from the Fermilab accelerator complex. A bunch of protons with a full width of about $250 \mathrm{~ns}$ is steered onto the production target. It consists of a tungsten $(\mathrm{W}) \mathrm{rod}, 160 \mathrm{~mm}$ long and with a $6.3 \mathrm{~mm}$ diameter, located in the middle of a high-field solenoid, the Production Solenoid, which is shown in Figure 1-5. The tungsten has been chosen as target material because of its thermal properties: the high melting point and the low thermal expansion coefficient allow to cool the target only by radiation. In the production target, $\mathrm{p}-\mathrm{W}$ interactions produce pions that are captured into helical trajectories in the field of the solenoid; these pions decay into muons that are also captured by the field of the solenoid. Mu2e collects the backscattered muon beam and transports it into the Transport Solenoid.

A thin window in the beginning of TS stops most of the antiproton contamination whilst transmitting almost all of the muons. The TS transmits low momentum negatively charged muons $(p<80 \mathrm{MeV} / \mathrm{c}$ ) into the Detector Solenoid, where they encounter the 34 thin $\mathrm{Al}$ foils that comprise the stopping target. The S-shape of TS suppress the line-of-sight neutral particles, while highly energetic negatively charged particles and positively charged particles are suppressed by several 
absorbers and collimators. In fact, a charged particle beam traversing a curved solenoid will drift perpendicularly to its axis with larger radius for higher momentum and with opposite direction for opposite charge sign.

Downstream of the DS stopping target is a tracking system and downstream of that is an electromagnetic calorimeter. In both of these devices, the inner region, out to a radius of about 38 $\mathrm{cm}$ is empty. This allows those muons that do not stop in the stopping target to pass through the detector to the muon beam dump. This also permits the low $p_{T}$ subset of the background particles to pass through the detector to the muon beam dump.

When a conversion or DIO electron is emitted from the stopping target, it travels in a helical trajectory and, if it has sufficient transverse momentum, $p_{T}$, its trajectory will be measured by the tracker. Only those electrons with $p_{T}>53 \mathrm{MeV} / \mathrm{c}$ will reach the tracker and only those with $p_{T}>90 \mathrm{MeV} / \mathrm{c}$ will intersect enough of the tracker to form a reconstructible track. Because almost all tracks from DIO have $p_{T}<m_{\mu} / 2 \simeq 60 \mathrm{MeV}$, a large fraction of them (97\%) do not reach the tracker. This is the key to making a measurement of $R_{\mu e}$ with a sensitivity of $O\left(10^{-17}\right)$ : the apparatus is only sensitive to the tail of the DIO energy distribution.
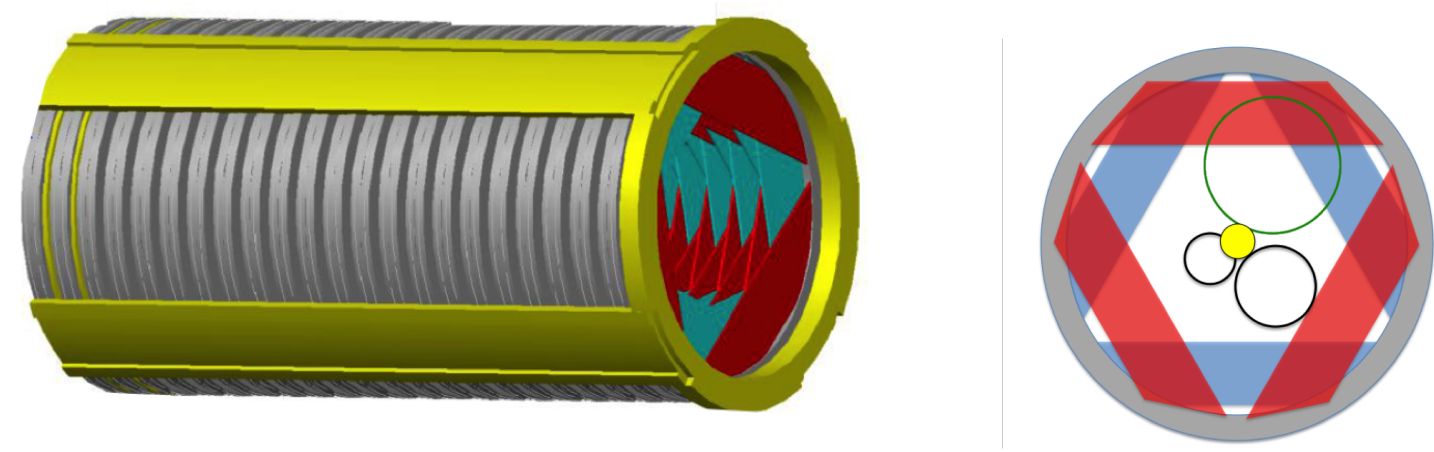

Figure 1-6: $3 D$ view of the Mu2e straw tube tracker (on the left) and cross sectional view of it with the trajectories of a $105 \mathrm{MeV}$ signal electron (in green), and a $53 \mathrm{MeV}$ background electron superimposed (lower right black circle). The yellow disk in the center is the stopping target. Background electrons with energy smaller than $53 \mathrm{MeV}$ (lower left black circle) miss the tracker entirely.

The tracking detector is made of low mass straw tubes oriented transverse to the solenoid axis. It consists of about 22000 straws grouped into 18 measurement stations, each made of two planes assembled to resemble an annular disk (Figure 1-6): all the electrons with $p<53 \mathrm{MeV}$ will pass through the central hole, increasing the tracker purity. The intrinsic resolution from the track fitting algorithm is very high. The momentum resolution is, in fact, dominated by fluctuations in the energy lost in the stopping target and antiproton absorber, by multiple scattering and by bremsstrahlung of the electron in the tracker. It is required to be better than $180 \mathrm{keV} / \mathrm{c}$.

The calorimeter (Figure 1-7) consists of 1860 CsI hexagonal crystals, arranged in two disks oriented transverse to the solenoid axis, positioned at a distance of $1 / 2$ pitch of a typical conversion 
electron to maximize acceptance. Lower energy electrons will pass through the hole, while the conversion electrons candidates will hit the upstream disk faces. The calorimeter provides timing and energy information important for forming a fast trigger and efficient particle identification. Moreover, it can help rejecting background from cosmic muons, which can be trapped in the DS or can generate an electron after the interaction with the detector material and cannot be vetoed by the Cosmic Ray Veto System (CRV). The CRV system covers all the DS and last part of TS (Figure 1-8), providing both a passive shielding (thick layer of concrete surrounding the DS) and an active veto, with a system of four layers of long scintillator strips with aluminum layer between them.

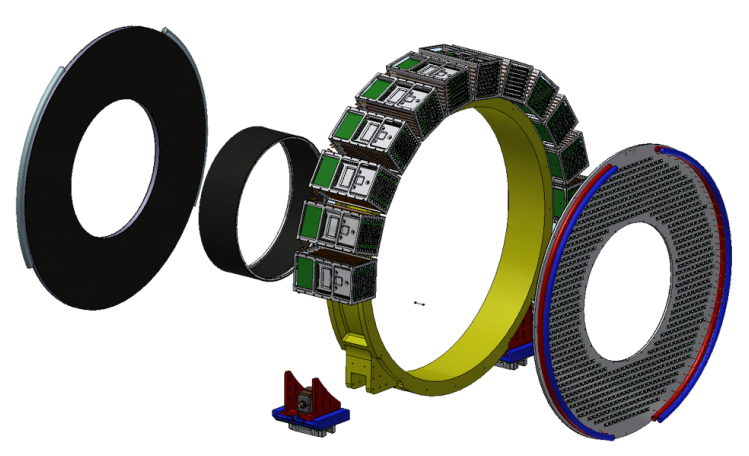

Figure 1-7: 3D view of the Mu2e calorimeter. The front-viewed annulus shows the crystals, the yellow annulus shows the outer bore with the readout boxes mounted on it, and the small annulus shows the inner bore. The supporting pads are shown in the bottom of the figure.

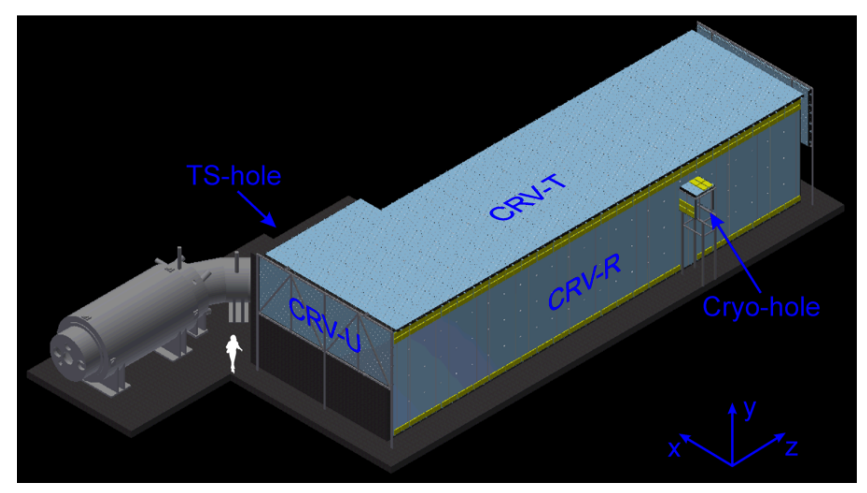

Figure 1-8: 3D view of the Mu2e Cosmic Ray Veto (CRV) (1-8). The blue box shows the passive shielding that covers TSd and DS, whereas TSu is shown on the left side. 


\subsection{Motion of CHARged PARTiCles in A SOLENOIDAL MAGNetiC}

\section{FIELD}

The motion of a charged particle with velocity $\vec{v}$ in a magnetic field $\vec{B}$ is governed by the Lorentz force law [Jackson(1999)]:

$$
\vec{F}=q \vec{v} \times \vec{B}
$$

Charged particles moving in a solenoidal field describe a helical trajectory created by the superposition of a circular motion with radius:

$$
r=\frac{p_{\perp}}{|q||\vec{B}|} \quad \text { or } \quad r(\mathrm{~mm})=3.3 \frac{p_{\perp}(\mathrm{MeV} / \mathrm{c})}{|q|(\mathrm{e}) B(\mathrm{~T})}
$$

where $q$ is the charge of the particle in units of elementary charge $e, p_{\perp}$ is the momentum component of the particle transverse to the direction of the field and $B$ is the magnitude of the field; and a drift motion described by the momentum component parallel to the direction of the field. The circular motion is called the Larmor motion of the particle and the radius is called the Larmor radius. When the axis of the solenoid has a curvature with radius vector $\vec{R}$, there is a drift motion developed normal to the bending plane, which displaces the particle trajectory with a velocity given by:

$$
\vec{v}_{R}=\frac{c^{2} p_{\|}^{2}}{q E} \frac{\vec{R} \times \vec{B}}{|\vec{R}|^{2}|\vec{B}|^{2}}
$$

where $E=\gamma m c^{2}$ is the total energy of the particle, $m$ is its mass and $p_{\|}$is the momentum component parallel to the direction of the magnetic field. An important aspect of the drift velocity is that it depends on the sign of the charge: it has opposite direction for particles with opposite charge. This property is used in the design of the Mu2e Transport Solenoid to filter out the unwanted positive particles from the negative particles actually used in the experiment, as explained below.

In a magnetic field with varying magnitude in space ("gradient field"), the force on a charged particle is given by:

$$
\vec{F}=-\mu \vec{\nabla}|\vec{B}|, \quad \mu=\frac{c^{2} p_{\perp}^{2}}{2 E|\vec{B}|}
$$

where $\mu$ is the magnetic moment of the particle in the field and $E$ is the total energy of the particle. This force, like the Lorentz force from which it is derived, does not do any work on the particle. Thus, the only effect is the change in the momentum direction, which accelerates the particle in the direction of the axis of the helix. If the gradient force acting on the particle is strong enough to change the direction of the particle, then a "magnetic mirror" effect is developed that reflects the direction of drift of the particle. This is an other property used in the design of the Mu2e transport 
system, as explained below.

\subsection{The Mu2E SOLENOID MAGNETS}

The solenoids perform several critical functions for the Mu2e experiment. Magnetic fields generated from these magnets are used to efficiently collect and transport muons from the production target to the muon stopping target while minimizing the transmission of other particles. Electrons are transported from the stopping target to detector elements where a uniform and precisely measured magnetic field is used to measure the momentum of electrons. The magnetic field values range from a peak of $4.6 \mathrm{~T}$ at the upstream end to $1 \mathrm{~T}$ at the downstream end. In between is a complex field configuration consisting of graded fields, toroids and a uniform field region, each designed to satisfy a very specific set of criteria.

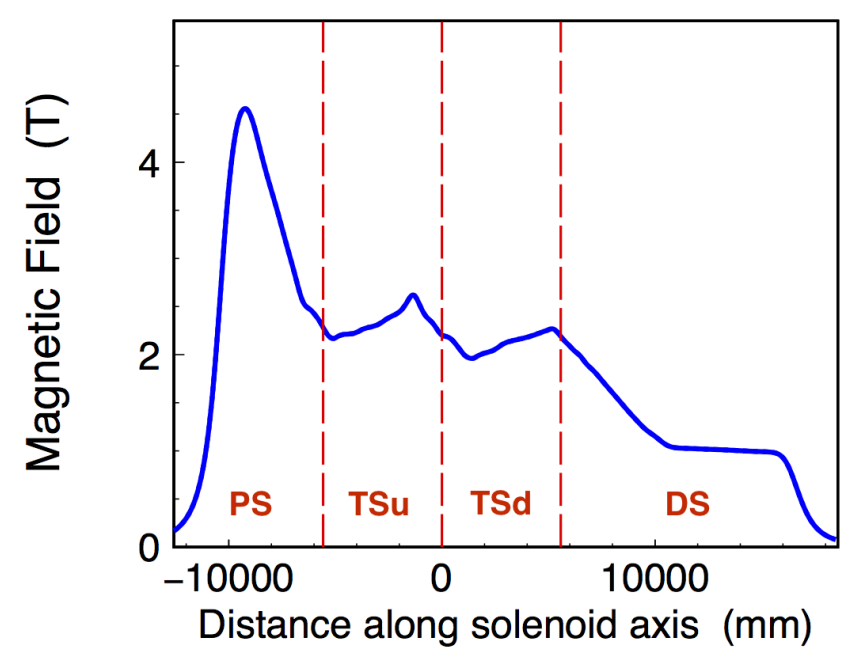

Figure 1-9: The Mu2e magnetic field along the solenoid axis (s), using the current Technical Division field maps. The red lines show the boundaries between the solenoids.

Mu2e creates its complex field configuration through the use of three magnetically coupled solenoid systems: the Production Solenoid (PS), the Transport Solenoid (TS) and the Detector Solenoid (DS). The Mu2e magnetic field created by the three solenoids is shown in Figure 1-9.

The PS field increases from 0 to $4.6 \mathrm{~T}$ in the proton direction, creating a positive field gradient which forms a magnetic mirror, as described in the previous section. This mirror boosts the particles towards the upstream exit of PS. A second magnetic mirror is created on the opposite side of the solenoid, where the field falls to about $2.5 \mathrm{~T}$. This negative field gradient reflects a portion of the pions and muons produced in the proton direction, increasing the yield of muons arriving at the stopping target in DS. 
The TS has an S-bend inducing a vertical drift that depends on the sign of the particles (see Equation 1.5). This allows, by appropriate placement of absorbers and collimators, the sign selection of the muon beam. In the upperstream region of TS (TSu) the field increases in order to shrink the Larmor radius of the particles, focusing the beam. After that, there is a sharper drop which kicks the particles through the collimator in the middle of TS. In TS downstream (TSd) the field rises again in order to create a narrow beam.

In the entrance of DS, the magnetic field is graded (from $2 \mathrm{~T}$ down to $1 \mathrm{~T}$ ) to form a magnetic mirror that reflects some backwards going electrons towards the tracker, thus increasing the acceptance. The decrease of the magnetic field helps also to reduce the background from high energy electrons coming from TS, increasing their pitch $p_{z} / p$ so that the drift along the $\mathrm{Z}$ axis is accelerated while the momentum magnitude $p$ remains unchanged. Thus, they go through the hollow part of the detector package. In the volume occupied by the detectors, the DS magnetic field is almost uniform at $1.0 \mathrm{~T}$ to allow for momentum reconstruction in the tracker, but still very slightly decreasing to move particles forward.

\subsubsection{Mu2e coordinate Systems}

Figure 1-10 shows the Mu2e coordinate systems that will be used in this study.

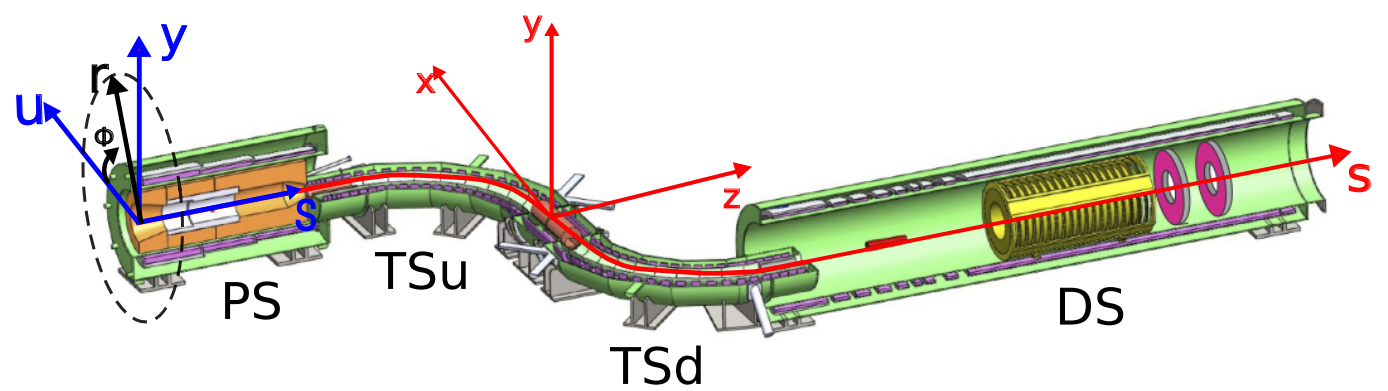

Figure 1-10: The Mu2e coordinate systems: the ( $\mathrm{u}, \mathrm{y}, \mathrm{s})$ local cartesian system (in blue), the (r, $\Phi, \mathrm{s})$ cylindrical system (in black) and the (X, Y, Z) Mu2e global cartesian coordinate system (in red), centered in the middle of the TS.

$(\mathbf{X}, \mathbf{Y}, \mathbf{Z})$ The Mu2e global cartesian system centered in the middle of TS. In PS and DS the Z coordinate is aligned with the solenoid axis. 
$(\mathbf{u}, \mathbf{y}, \mathbf{s})$ Coordinate system attached to the Mu2e axis, with the s axis tangent to the symmetry axis (i.e., for PS and DS, parallel to the $Z$ axis in the Mu2e coordinate system), the $y$ axis pointing vertical up (i.e. parallel to the $\mathrm{Y}$ axis in the Mu2e coordinates everywhere), and the $\mathrm{u}$ axis completing a right-handed cartesian system. In PS and DS, this local system is simply parallel-translated w.r.t. the global Mu2e system in the XZ plane, to have the origin on the Mu2e symmetry axis. In TS, it is both translated and rotated in the XZ plane. In the straight section of TS, the positive $\mathrm{u}$ axis becomes parallel to the Mu2e positive $\mathrm{Z}$ axis and the positive $\mathrm{s}$ axis becomes parallel to the Mu2e negative $\mathrm{X}$ axis.

$(\mathbf{s}, \Phi, \mathbf{r})$ The s is defined as above, $\mathrm{r}$ is the coordinate transverse to s and $\Phi$ is the rotation angle (azimuth) around the axis. $\Phi$ is defined so that $\Phi=0$ corresponds to the $+\mathrm{u}$ axis.

\subsubsection{Production and Detector Solenoids}

The Production Solenoid is a high field magnet with a graded solenoidal field varying smoothly from 4.6 Tesla to 2.5 Tesla. The gradient will be formed by 3 axial coils with a decreasing number of windings, made of aluminum stabilized $\mathrm{NbTi}$. The solenoid is approximately $4 \mathrm{~m}$ long with an inner bore diameter of approximately $1.5 \mathrm{~m}$ that is evacuated to $10^{-4}$ Torr.

The Production Solenoid is designed to capture pions and the muons into which they decay and guide them downstream to the Transport Solenoid. This process is initiated by $8 \mathrm{GeV}$ protons striking a production target near the center of the Production Solenoid. Remnant protons that are not absorbed by the target and very forward-produced secondary particles exit at the high field end of the solenoid. Pions in the forward direction with angles greater than $\sim 30^{\circ}$, relative to the solenoid axis, are reflected back by the higher field and move along with the backward produced particles in helical trajectories towards the Transport Solenoid.

The Detector Solenoid is a large, low field magnet that houses the muon stopping target and the detectors required to identify and analyze conversion electrons from the stopping target. It is nearly $11 \mathrm{~m}$ long with a clear bore diameter of about $2 \mathrm{~m}$. The muon stopping target resides in a graded field that varies from $2 \mathrm{~T}$ to $1 \mathrm{~T}$. On the TS side of the muon stopping target, the graded field captures conversion electrons that are emitted in the direction opposite the detector components causing them to reflect back towards the detector. On the other side of the stopping target, the graded field focuses electrons toward the tracker and calorimeter. The graded field also plays an important role in reducing background from high energy electrons that are transported to the Detector Solenoid by steadily increasing their pitch as they are accelerated towards the downstream detectors. The resulting pitch angle of these beam electrons is inconsistent with the pitch of a conversion electron from the stopping target.

The actual detector components reside in a field region that is relatively uniform. The field in 
this region has two requirements. First, the magnetic field is important for the measurement of the electron momentum and energy. The tracker-determined trajectory, along with the magnetic field map, determines the electron momentum. This requires the magnetic field to be as uniform as possible. The tracker-determined trajectory is then extrapolated into the downstream calorimeter and matched with calorimeter energy deposition. The energy/momentum match further validates the electron identification. The second design consideration is that local field minima are a potential source of backgrounds, because muons can be trapped in the regions of those minima and decay late (within the live window), thus producing signal-like electrons into the detector. This problem is mitigated by superimposing a small negative axial gradient in the tracker and calorimeter region.

\subsubsection{Transport Solenoid}

The S-shaped Transport Solenoid consists of a set of superconducting coils forming a magnetic channel that efficiently transmits low energy negatively charged muons from the Production Solenoid to the Detector Solenoid. Negatively charged particles with high energy, positively charged particles and line-of-sight neutral particles are nearly all eliminated by absorbers and collimators before reaching the Detector Solenoid. Selection of negatively charged muons is accomplished by taking advantage of the fact that a charged particle traversing a toroidal field will drift perpendicularly to the toroid axis, with positives and negatives drifting in opposite directions (see Equation 1.5). Most of the positively charged particles are absorbed in the central collimator.

The Transport Solenoid consists of five distinct regions: a $1 \mathrm{~m}$ long straight section (TS1), a $90^{\circ}$ curved section (TS2), a second straight section about $2 \mathrm{~m}$ long (TS3), a second $90^{\circ}$ curved section (TS4) that brings the beam back to its original direction, and a third straight section (TS5) of $1 \mathrm{~m}$ length. The major radius of the two curved sections is about $3 \mathrm{~m}$ and the resulting total magnetic length of the Transport Solenoid along its axis is about $13 \mathrm{~m}$. The inner warm bore of the Transport Solenoid cryostat has a diameter of about $0.5 \mathrm{~m}$. The Transport Solenoid is shown in Figure 1-11.

Particles produced with a small pitch in a uniform field region can take a very long time to progress down the beamline toward the muon stopping target. To suppress background from these late arriving particles, the three straight sections in the Transport Solenoid employ negative axial gradients for radii smaller than $0.15 \mathrm{~m}$. The radius is set by the geometry of the beam collimators. This requirement is intended to eliminate traps, where particles bounce between local maxima in the field until they eventually scatter out and travel to the Detector Solenoid where they arrive late and may cause background.

The requirement on a negative gradient is relaxed in the curved sections of the TS because they are toroidal sections. In fact in a toroid region, spiraling particles drift up or down depending on the sign of their charge, with a displacement that is proportional to their momentum and inversely proportional to their pitch. Particles with small pitch progress slowly through the toroid and drift 


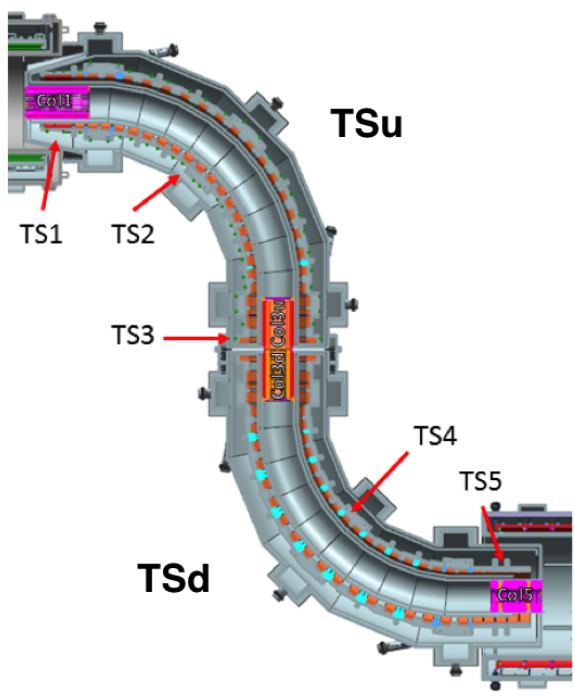

Figure 1-11: The Transport Solenoid consists of a set of superconducting coils forming a magnetic channel that efficiently transmits low energy negatively charged muons from the Production Solenoid to the Detector Solenoid.

to the wall where they are absorbed.

\subsection{SOURCES OF UNCERTAINTY IN THE Mu2E MAGNETIC FIELDS}

The magnetic design of the solenoid system defines the coil positions and angles under cold and powered operating conditions. However, thermal contraction and magnetic forces will alter those positions and angles from the room temperature assembly. Therefore, it is really important to verify if there will be any changes in the survey and how these could affect the Mu2e physics goals.

This thesis presents a study of possible sources of uncertainty in the Mu2e magnetic fields: these can be misalignments of the coils caused by mechanical and magnetic forces and approximations in the field calculation. TS misalignments can be generated by rotations of the coils or parallel coil displacements. The following chapters describe in detail how the variations in the magnetic field are computed and what their effects on the muon and pion stopping rates and on beam electron background are.

Another potential source of uncertainty is determined by current variations. However, such variations do not affect the shape of the field along the beamline, which is the major concern for the experiment, because the coils are powered up sequentially. Therefore, these fluctuations are propagated to the field coherently, altering only its overall scale. 


\subsection{The Mu2e Simulation PaCKage}

The Mu2e simulation software package is used to compute the background yields and signal efficiency described below. The Mu2e simulation software is built on the GEANT4 solid geometry and physics interaction software library [Agostinelli et al.(2003)]. GEANT4 is a toolkit for simulating the passage of particles through matter, using Monte Carlo methods. Implemented in $\mathrm{C}++$ programming language, it includes a complete range of functionality including tracking, geometry, physics models and particle recording. The physics processes offered cover a comprehensive range, including electromagnetic, hadronic and optical processes, decays of long-living particles, material and elements, over a wide energy range. Using the Mu2e simulation package, all components of the Mu2e experiment can be constructed in the simulation environment. Particle interactions with matter are simulated, and track reconstruction is conducted.

\subsubsection{ART and ROOT output files}

The Mu2e Offline software is written as a set of plugins that can be run by a framework named art, which is developed and supported by the Fermilab Scientific Computing Division (SCD) and used by many of the Intensity Frontier experiments at Fermilab. A software framework is an abstraction in which software providing generic functionality can be selectively changed by additional user-written code, thus providing application-specific software. art is a command-linedriven event-processing framework application, which means that the application is not interactive and that the program processes a sequence of events, as specified by the user.

Written in $\mathrm{C}++$, it has been designed for use in most places that a typical HEP experiment might require a software framework, including high-level software triggers, online data monitoring, calibration, reconstruction, simulation and analysis.

Run-time configuration for art is written in the Fermilab Hierarchical Configuration Language (FHiCL), a language that was developed at Fermilab to support run-time configuration for several projects, including art. A FHiCL file contains definitions of $\mathrm{C}++$ classes that implement the art services. Algorithms (simulations, reconstructions or just analysis code) are built into these classes and put into dynamic libraries called modules. The FHiCL files declare what modules will be loaded, in what order they are to run and what files will be read and written.

A simulation is run using a FHiCL file, which specifies the required modules and call files containing geometry and physics information for the simulation. art runs the modules and produces events, that are just organized collections of data products, with informations about them. These events are pulled out as art files, that can be converted in flat ROOT ntuples. The custom data analysis and graphics package ROOT from CERN ([Brun and Rademakers(1997)]) has been used to analyze the results of the simulations. 


\subsubsection{Stage 1,2 and 3 simulations}

To make it practical to perform optimizations of the detector and shielding geometry and materials, the Mu2e simulations have been subdivided into several stages. In this way, simulation parameters that alter processes taking place in DS can be changed without having to repeat the simulation of all processes that take place from PS through DS. The first stage begins the simulation with $8 \mathrm{GeV}$ protons interacting with the production target and tracks all produced particles to the mid-point of the transport solenoid. Location, arrival time, and four-momenta of any particle making it that far are saved. The second stage begins with the output of the first stage and tracks particles to the entrance of the detector solenoid. The third stage propagates the surviving particles from stage two through the upstream portion of the detector solenoid vacuum and records muon and pion stops in the aluminum capture target. Particles that intersect the tracker or calorimeter are recorded. The fourth and final stage describes the complete Mu2e setup and the particle reconstruction through the tracker and calorimeter. In this manner the amount of CPU necessary to study variations in the tracker, for example, is significantly reduced, since only the fourth stage would have to be re-run. This is much faster than re-doing simulations from primary protons. In addition, the stage approach allows to employ resampling techniques where appropriate, significantly increasing the effective statistics of the sample in a very CPU efficient manner. Stages 1 and 2 do not track electrons below $1 \mathrm{MeV}$. In the subsequent stages all particles are simulated regardless of their energy.

\subsection{Thesis OUTLINE}

Mu2e is designed to operate at certain conditions that define the nominal operating point. These conditions control the precision of the experiment and are largely specified by the magnetic fields of the solenoids. Because of the tight precision requirements, in order to achieve an improvement of four orders of magnitude in the current limits on charged lepton flavor violation, it is necessary to examine how the physics parameters determining the precision of the experiment are changed when the conditions shift from the nominal operating point due to instrumental uncertainties, in particular those affecting the fields of the solenoids. This thesis explores the precision tolerance of the experiment to misalignments of the Transport Solenoid, the instrument performing negative muon selection in the appropriate momentum range and steering them to the muon capture target. Such misalignments are among the most important sources of instrumental uncertainties. The exploration is done by examining how far these misalignments can bring the conditions of the experiment from the nominal operating point and how such misalignments can be detected and quantified. To achieve this objective, misalignments of the coils due to mechanical and magnetic forces are considered. This thesis then examines the effect of field uncertainties on muon and pion 
stopping rates on the target, on beam electron background and the case of testing the Transport Solenoid field with low momentum electrons as an alternative to measuring it with field probes.

Chapter 2 describes in detail the magnetic field of the Mu2e solenoid system, introducing the concept of field maps. Comparisons between different field maps are reported, using ROOT software tools. In these comparisons, different designs of the solenoids were examined and validated. The interpolation algorithms used to evaluate the field at given points in space are described, and discrepancies in the magnetic fields due to approximations in the field calculation are studied. Moreover, the SolCALC software in MATLAB language is used to create varied maps, containing TS rotations or parallel coil displacements. Field maps with TS rotations of $0.15^{\circ}$ and $0.50^{\circ}$ are described and compared with the default magnetic field maps provided by the Technical Division of Fermilab.

The effects of TS rotations and parallel coil displacements on the muon and pion stopping rates are reported in Chapter 3. Chapter 4 contains results from expected beam electron background. The study is done using all the varied maps, in order to estimate possible variations in this background due to field uncertainties. The last part of the study involves the idea of testing the TS field using low momentum electrons. This low-momentum electron test is simulated for different configurations of field, source locations and vacuum (Chapter 5). Chapter 6 summarizes the results of these three studies, quantifying the tolerance of the experiment to field uncertainties and describing the perspectives of the low momentum electron test of the Transport Solenoid. 


\section{CHAPTER 2}

\section{MAgNeTiC FIELD CALCULATIONS}

\subsection{Mu2E FIELD MAPS}

The magnetic field of each solenoid is mapped at the discrete points of a 3D grid, which is called Field Map. The Mu2e code reads tables of values of the magnetic field components from the field maps, and then computes the values at any given point in space applying $3 \mathrm{D}$ interpolation.

Each map describes the field in a region of the muon beamline: there are four maps describing the magnetic field in PS, TSu, TSd and DS, as illustrated in Figure 2-1. The blue lines enclose the entire solenoid structure: the coils, the cryostat and the vacuum bore inside which the particles are transported. The regions covered by the maps include the vacuum volume, the surrounding coils and a small amount of the space surrounding that. The four field map regions touch each other but do not overlap. There is a second group of field maps, that extend the field map from PS to the proton dump and extinction monitor areas.

The field maps are provided in a text format that consists of a header and a table containing the values of $\mathrm{x}, \mathrm{y}, \mathrm{z}, B_{x}, B_{y}$ and $B_{z}$, where the positions are given in $\mathrm{mm}$, in the Mu2e coordinate system (see Figure 1-10), and the field values in T. The header contains information about the size and granularity of each map: i.e the lower limits, the number of points, and the spacing in each coordinate. It also contains information regarding whether the map is assumed to be symmetric with respect to the $\mathrm{XZ}$ plane. This symmetry holds in the case where the solenoids are modeled with ideal coils (cylindrical rings with uniform current density). For maps assuming XZ-plane symmetry, the field values are given only at points for $Y \geq 0$ (one-sided maps). To expand the 


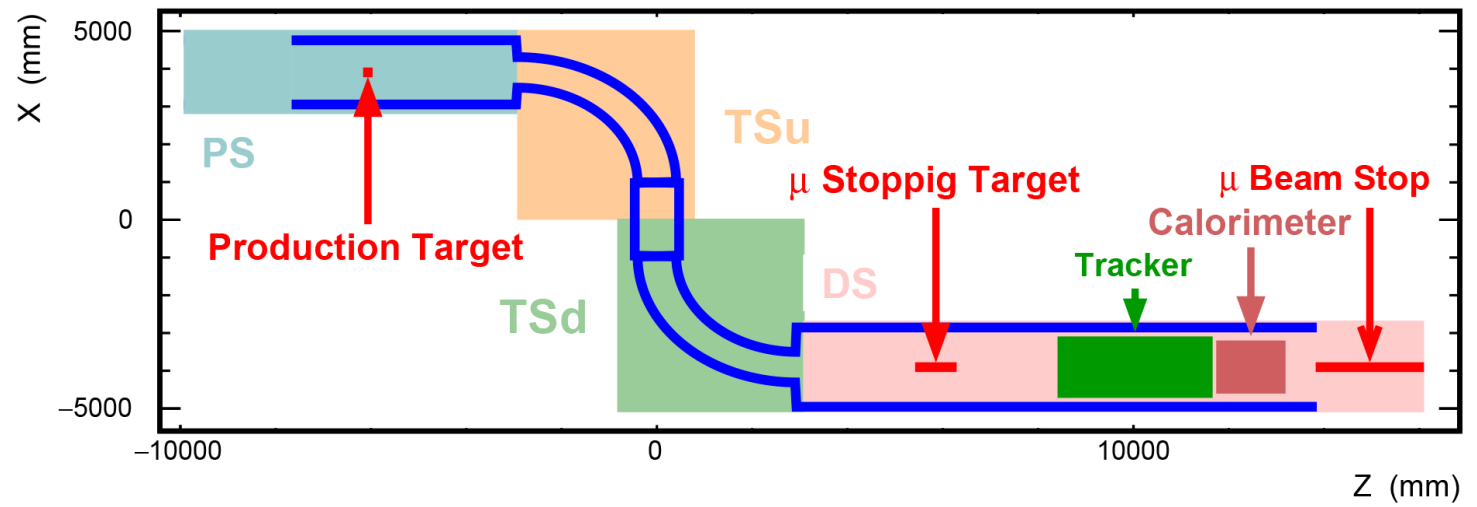

Figure 2-1: Representation of the Mu2e solenoids (in blue). The colored boxes represent the field maps for PS, TSu, TSd and DS. Note that the maps are bigger than the area covered by the solenoids. The coordinate system is the Mu2e cartesian coordinate system, centered in TS3. The figure also shows the basic elements of the experiment.

maps for negative $\mathrm{Y}$, the field is reflected with respect to the $\mathrm{XZ}$ plane using:

$$
\begin{aligned}
B_{x}(X,-Y, Z) & =B_{x}(X, Y, Z) \\
B_{y}(X,-Y, Z) & =-B_{y}(X, Y, Z) \\
B_{z}(X,-Y, Z) & =B_{z}(X, Y, Z)
\end{aligned}
$$

Double-sided maps contain double number of points, since they do not assume XZ-plane symmetry and therefore the field values are given at all $\mathrm{Y}$ points. One-sided maps are used to describe solenoid magnetic field with split coils, while double-sided maps describe magnets with helical model.

Mu2e event simulation and reconstruction software reads field maps in binary format. In fact, the Mu2e simulation software spends about half of the total CPU time to compute the field at the required points along the simulated trajectories. It is therefore important to speed up this part of the computation, which is achieved by using the binary format instead of the text format. For each text file, the binary format consists of two files that have the same base name as the text file, with the extension .header and bin. The header file is simply a copy of the header information from the text file, while the .bin file contains the field values at the grid points in binary format; it is simply an image of these numbers in the program memory. 


\subsection{THREE-DIMENSIONAL INTERPOLATION ALGORITHMS}

Mu2e event simulation and reconstruction software knows the magnetic field only at the points of the field maps of each solenoid. The field at any other point is computed by piecewise continuous 3D interpolation, where no continuity is enforced across grid cells or maps. Currently, there are two interpolation methods available in Mu2e-Offline: tri-linear (default) and tri-quadratic. Both algorithms are constructed on the basis of the Lagrange interpolation formula:

$$
\begin{aligned}
P(x)= & \frac{\left(x-x_{2}\right)\left(x-x_{3}\right) \ldots\left(x-x_{n}\right)}{\left(x_{1}-x_{2}\right)\left(x_{1}-x_{3}\right) \ldots\left(x_{1}-x_{n}\right)} y_{1}+\frac{\left(x-x_{1}\right)\left(x-x_{3}\right) \ldots\left(x-x_{n}\right)}{\left(x_{2}-x_{2}\right)\left(x_{2}-x_{3}\right) \ldots\left(x_{2}-x_{n}\right)} y_{2} \\
& +\cdots+\frac{\left(x-x_{1}\right)\left(x-x_{2}\right) \ldots\left(x-x_{N-1}\right)}{\left(x_{N}-x_{1}\right)\left(x_{N}-x_{2}\right) \ldots\left(x_{N}-x_{N-1}\right)} y_{N}
\end{aligned}
$$

which describes the interpolating polynomial of degree $N-1$ through $N$ points $y_{1}=f\left(x_{1}\right)$, $y_{2}=f\left(x_{2}\right), \ldots, y_{N}=f\left(x_{N}\right)$. There are $N$ terms, each a polynomial of degree $N-1$ and each constructed to be zero at all the $x_{i}$ except one, at which it is constructed to be $y_{i}$. The algorithm resulting by the implementation of the Lagrange formula gives no error estimate.

In the case of trilinear interpolation $N=1$ : it is the extension of linear interpolation, which operates in spaces with dimension $D=1$, and bilinear interpolation, which operates with dimension $D=2$, to dimension $D=3$. It requires $(1+N)^{D}=8$ adjacent pre-defined values surrounding the interpolation point. The Lagrange formula will be integrated three times, along the three axes $\mathrm{x}, \mathrm{y}$ and $\mathrm{z}$. The result of trilinear interpolation is independent of the order of the interpolation steps along the three axes. For a given point in the space, the trilinear interpolation algorithm takes the nearest 8 grid points surrounding it and approximates the value of the intermediate point $(\mathrm{x}, \mathrm{y}, \mathrm{z})$ within the local axial rectangular prism linearly, using data on the cell points. In the tri-quadratic interpolation algorithm 4 adjacent cells of the grid are required, for a total of 27 grid points surrounding the interpolation point.

The linear interpolation error is bounded according to the following formula:

$$
|f(x)-g(x)| \leq C\left|x_{i}-x_{i-1}\right|^{2}
$$

where $C=\frac{1}{8} \max _{x \in \Delta x}\left|g^{\prime \prime}(x)\right|, f(x)$ and $g(x)$ are the interpolated and original (true) functions, respectively. When the spacing between grid points grows or the second derivative $g^{\prime \prime}(x)$ of the function becomes locally large, the approximation becomes worse. In the present case, given the coil dimensions, a grid spacing of $25 \mathrm{~mm}$ is a reasonable choice for the design derivatives of the field. 


\subsection{FIELD MAP TESTS}

\subsubsection{Map validations}

The Mu2e solenoids will be constructed by two different vendors: one is in US (General Atomic, GA), which will build PS and DS, and one in Italy, which will build TS. Initially, the field maps for all solenoids were provided by the Fermilab Technical Division (TD), which made the original design of all solenoids. The version of TD maps used for the TDR simulations (Mau9) was the default set in the beginning of the studies presented here. Later on, new maps were provided by TD (Mau10). Consequently, there are two sets of maps available, from TD and from GA, which have to be tested and validated, based on their respective specifications, by direct comparisons of the fields that they describe.

Both TD and GA maps are computed by using OPERA3D [Fields(2012)], a commercial software to compute magnetic fields in 3D. The field maps provided by TD describe all magnets as ideal solenoids, thus they assume the XZ-plane symmetry and are one-sided maps. They will be indicated from now on with the name Mau. There are several variants of Mau maps: in this study Mau9 and Mau10 have been used, while the current version used in Offline is Mau10. Mau9 and Mau10 differ by a slight change in the geometry and position of a few coils in TS1, TS3 and TS5 regions (see subsection 1.3.3), to conform with mechanical aspects of the housing supports or the interference with the cryostat.

Different field maps have been provided by GA (labeled with a number which specifies the version). They differ in the XZ-plane symmetry assumption (one-sided if symmetry is assumed, two-sided if not) and model of DS magnet (split or helical coils). Table 2.1 lists all the Mau and GA field maps and their properties.

Table 2.1: List of GA and Mau field maps. GA00 is the equivalent to the Mau9 maps, used as benchmark for the Null Test (checks if the fields computed with the sets of the maps are numerically identical at all points of the grid). The one-sided maps give field values just for $Y \geq 0$ (assuming XZ-plane symmetry), while double-sided maps are given for positive, zero and negative Y. DS coils can be split (ideal solenoid model) or helical (helical winding model). GA04 and GA05 differ for the location of a few coils. The GA05 map starts from the model of GA04; the placement of some coil positions at large $z$ is modified in order to ensure negative gradient out to larger radii in the detector region.

\begin{tabular}{l||cc}
\hline \hline & Map extension & DS Coils \\
\hline GA00 & Double-sided & Split \\
GA01 & One-sided & Split \\
GA02 & Double-sided & Split \\
GA03 & Double-sided & Helical \\
GA04 & Double-sided & Helical \\
GA05 & Double-sided & Helical \\
\hline \hline
\end{tabular}

\begin{tabular}{l||cc}
\hline \hline & Map extension & DS Coils \\
\hline Mau9 & One-sided & Split \\
Mau10 & One-sided & Split \\
\hline \hline
\end{tabular}


In order to compare the field maps of the Mu2e solenoids, Root tools have been developed. They are designed to use two sets of maps, where each set has a map for each solenoid (PS, TSu, TSd, DS). Each set may or may not assume XZ-plane symmetry and, depending on the assumption, it is used accordingly. Using these tools, numerical comparisons have been made between the field maps provided by GA and TD. The first step is to compare the GA maps which use the DS solenoid model (i.e. GA00, GA01, GA02) with Mau9 maps: this is the benchmark (Null Test) because it is expected to find differences well below the design tolerance $\left(\leq 10^{-4} \mathrm{~T}\right)$, since both the maps use the same magnetic model. The two sets of maps should agree at this level of precision for the $G A$ helical model to be reliable. After the Null Test, if GA with DS split coils is found consistent with Mau9, it will be used as reference for other comparisons with the GA maps assuming DS helical model. The comparisons made are:

1. GA01 vs Mau9 (1 $1^{\text {st }}$ Null Test);

2. GA03 vs Mau9 ( $1^{\text {st }}$ Helical Test);

3. GA03 vs GA02 (2 ${ }^{\text {nd }}$ Helical Test);

4. GA00 vs Mau9 (2 ${ }^{\text {nd }}$ Null Test);

5. GA04 vs GA00 (3 ${ }^{\text {rd }}$ Helical Test);

6. GA05 vs GA00 ( $4^{r d}$ Helical Test).

The first three numerical comparisons revealed inconsistencies between Mau9 and GA calculations, eventually traced to the fact that the two maps had a few coils in slightly different positions. For this reason GA provided two more maps with the inconsistencies fixed: GA00, used for Null Test with Mau9 maps, and GA04, identical to Mau9 except new DS model, which has to be compared with GA00.

Figure 2-2 shows 3D plots for the field magnitude differences between GA00 and Mau9 maps. The differences are shown as functions of the path length s parallel to the geometric axis and the radial distance $r$ (equals to $\mathrm{u}$ or $\mathrm{y}$ in the $\mathrm{XZ}$ or $\mathrm{YZ}$ plane, respectively) from the geometric axis (see 2-2(a) and 2-2(b)).

Figure 2-3 shows plots for the GA00 vs Mau9 comparison only on the magnetic axis, along all the path length $s$ (from the entrance of PS through the exit of DS). The dotted vertical lines denote, from left to right, the boundaries between PS, TSu, TSd and DS. Figures 2-3(a), 2-3(b), 2-3(c) and $2-3(\mathrm{~d})$ are made using interpolated field values along certain paths parallel to the geometric axis, while figures 2-3(e) and 2-3(f) are made using the original values form the field maps at all grid points. Only a limited set of interpolated plots is shown, corresponding to the case of zero radius (along the beam axis). In general, the test involves groups of plots at four values of 

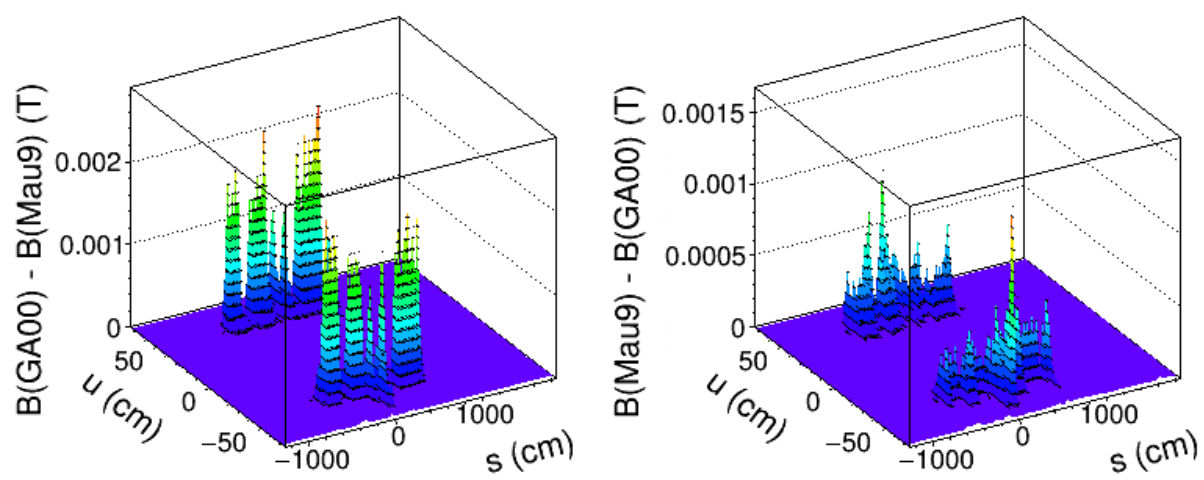

(a) 3D field magnitude differences in the XZ plane.
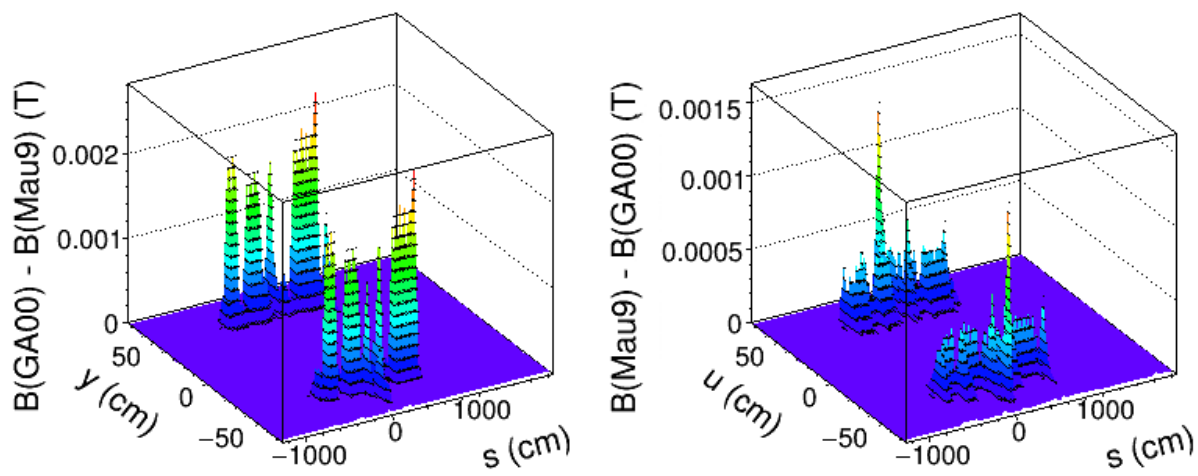

(b) 3D field magnitude differences in the YZ- plane.

Figure 2-2: $3 D$ comparisons between Mau9 and GA00 (Null Test) field maps. The magnetic field difference in T between the two maps is represented in 3D, as function of the path length s (from the entrance of PS through the exit of DS) and the transverse coordinate ( $u$ or $y$ ) in the local coordinate system (Figure 2-1). For each figure, the plot on the left represents the positive difference between the GA00 and Mau9 fields, while that on the right represents the negative difference between GA00 and Mau9.

the azimuth per group, over a family of radii that are monitored by the input. In figures 2-3(a), 2-3(b) and 2-3(c) the reference field (dashed lines) and the test field (solid line) overlap, so that the differences between the two fields are not visible. The nearly flat segments in the DS region in figure 2-3(a) correspond to the detector fiducial volume, where the field has to be uniform in order to analyze the momentum of the conversion electrons; the same region is evident in 2-3(b), where the derivative is nearly 0 . In that region, there is a very slow drop of the field, to keep the particles moving forward while allowing, at the same time, the momentum analysis of the detected particles, which requires a field as uniform as possible. The plot of signed field differences in figure 2-3(d) points out that the bigger differences are in the TS region. Investigations of the plots at finite radii showed that they are most prominent close to the coils $(\mathrm{r}=40 \mathrm{~cm})$ at the TS boundaries. The differences are generally observed at the level of $10^{-4}$, except in the TS region near the coils where they go up to the level of $10^{-2.5}$. 


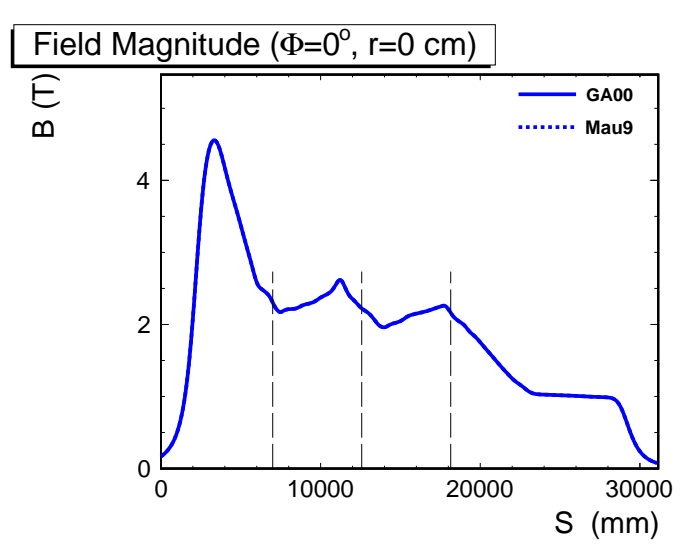

(a) Comparisons of the magnitude of the magnetic field as functions of the path length s. The flat area in the DS region corresponds to the detector fiducial volume.

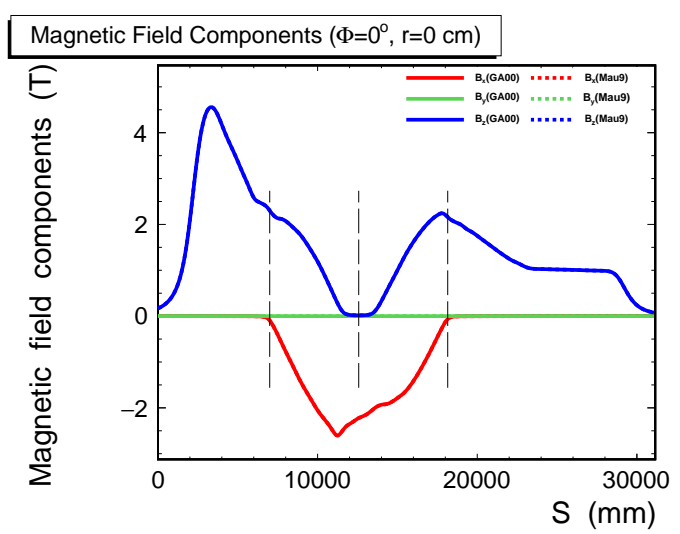

(c) Comparisons of the magnetic field components as functions of the path length.

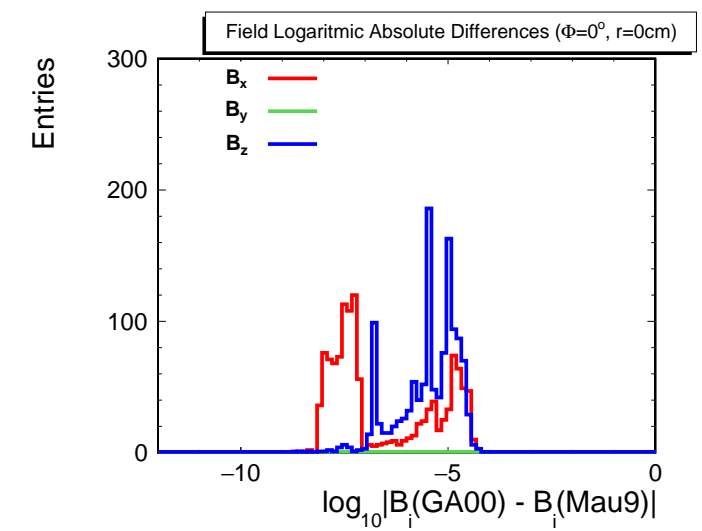

(e) Histogram of the decimal logarithm of the absolute differences of the magnetic field components.

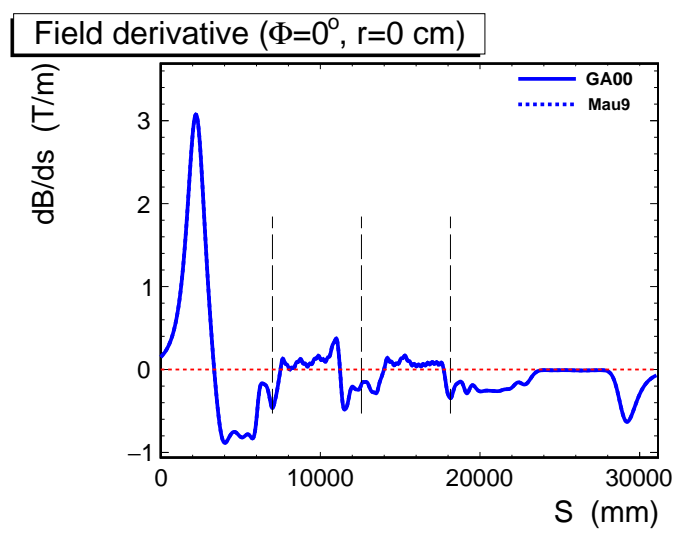

(b) Comparisons of the derivative of the magnetic field with respect to and as function of the path length s. The 0 derivative area in the DS region corresponds to the detector fiducial volume.

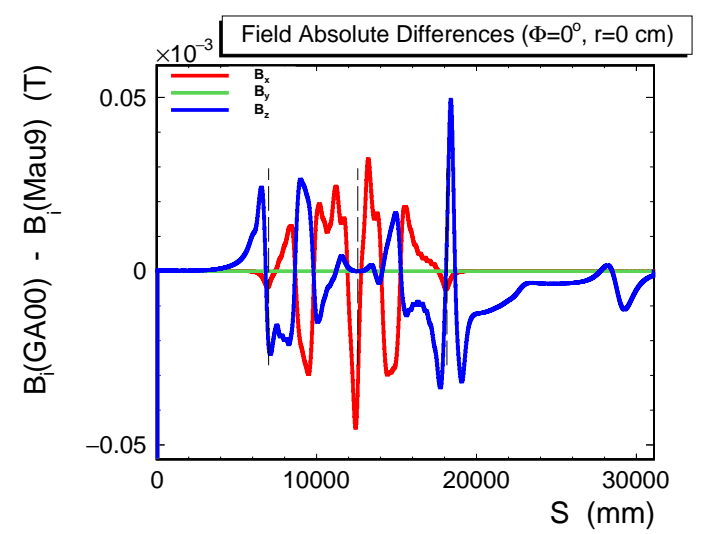

(d) Plots of the signed differences of the magnetic field components.

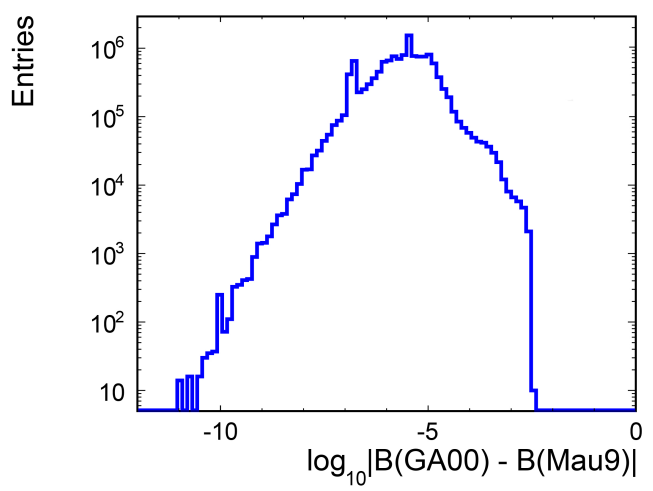

(f) Histogram of logarithmic absolute differences of the field magnitude at all grid points.

Figure 2-3: Comparisons between Mau9 and GA00 (Null Test) field maps, on the magnetic axis, along all the path length s (from the entrance of PS through the exit of DS). Dotted vertical lines denote the boundaries of PS, TSu, TSd and DS regions. 2-3(a), 2-3(b), 2-3(c) and 2-3(d) are made using interpolated field values along certain paths parallel to the geometric axis, while 2-3(e) and 2-3(f) are made using the original values form the field maps at all grid points. In 2-3(a),2-3(b) and 2-3(c) the reference field is represented by a dashed lines, while the test field with a solid line. In cases the dashed line is not visible, it is covered by the corresponding solid line. 
From the highest bin of the logarithmic histogram (2-3(d)) we can inspect how strong the largest differences are. We can look for the grid points where the largest differences occur, using scatter plots in the $(u, y, s)$ and $(r, \Phi, s)$ local coordinate systems. Figure 2-4 shows the scatter plots for the DS magnet: no significant differences are observed in the active volume of the detector, so that there are no effects on physics. The main significant differences $(>1 \mathrm{G})$ in $|\mathrm{B}|$ are detected near the coils: this is probably associated with details of numerical integration.
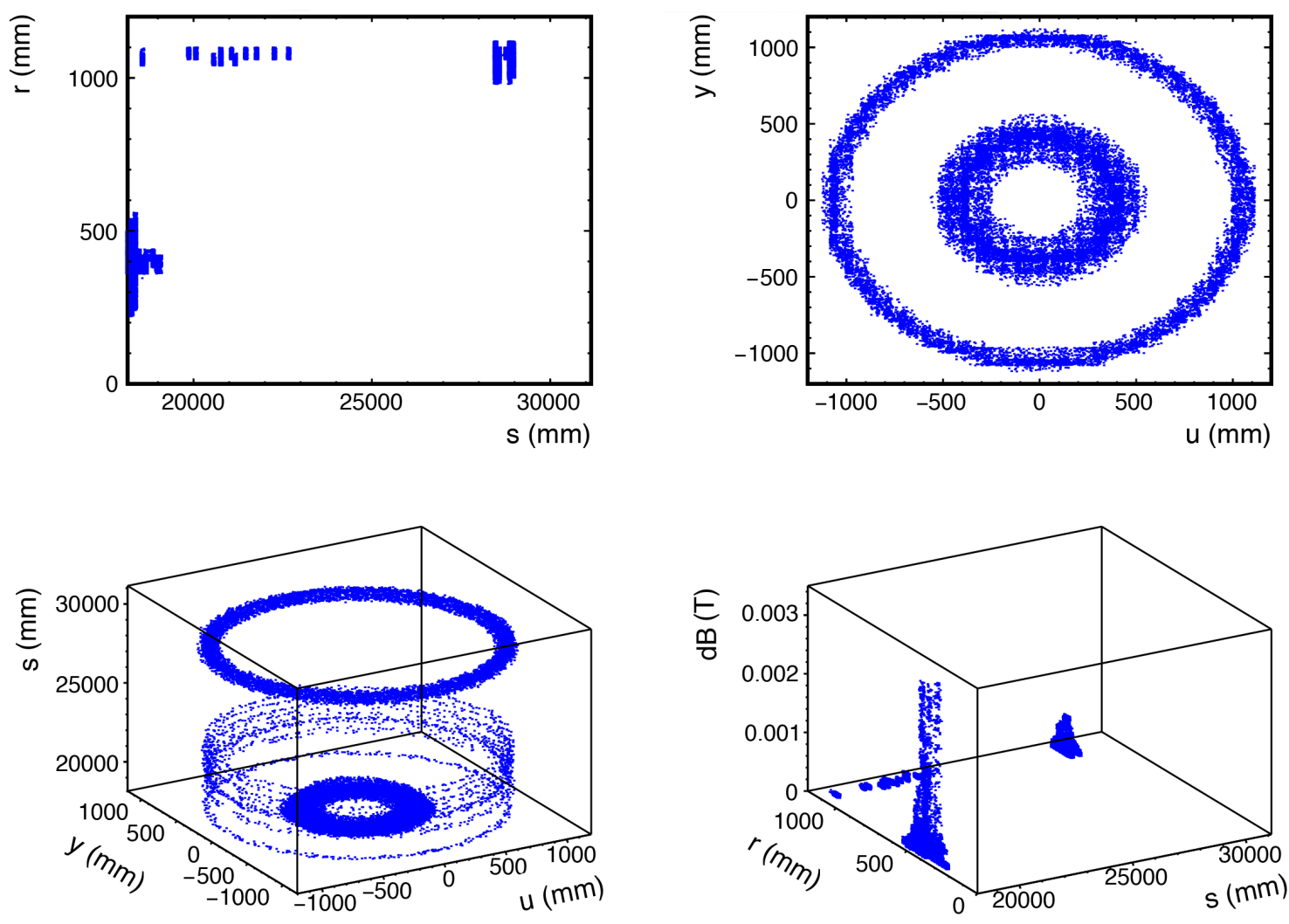

Figure 2-4: Scatter plots of DS grid points where the field magnitude differs by more than $1 \mathrm{G}$ between the GA00 and the GA05 DS maps. The coordinate systems used are $(u, y, s)$ and $(r, \Phi, s)$.

Figure 2-5 shows comparisons between the GA00 and GA05. Overall consistency between the two calculations is observed, except in the DS region. These differences are expected because of the different DS model (split coils in GA00, helical coil in GA05). The biggest difference is observed near the exit of DS where the field drops to zero, pointing to a slightly different turn-off curve.

\subsubsection{Algorithm tests}

It is important to know how the raise of the order of interpolation can affect the precision of the calculated field and how well the interpolation can reproduce known field values. Thus, a 

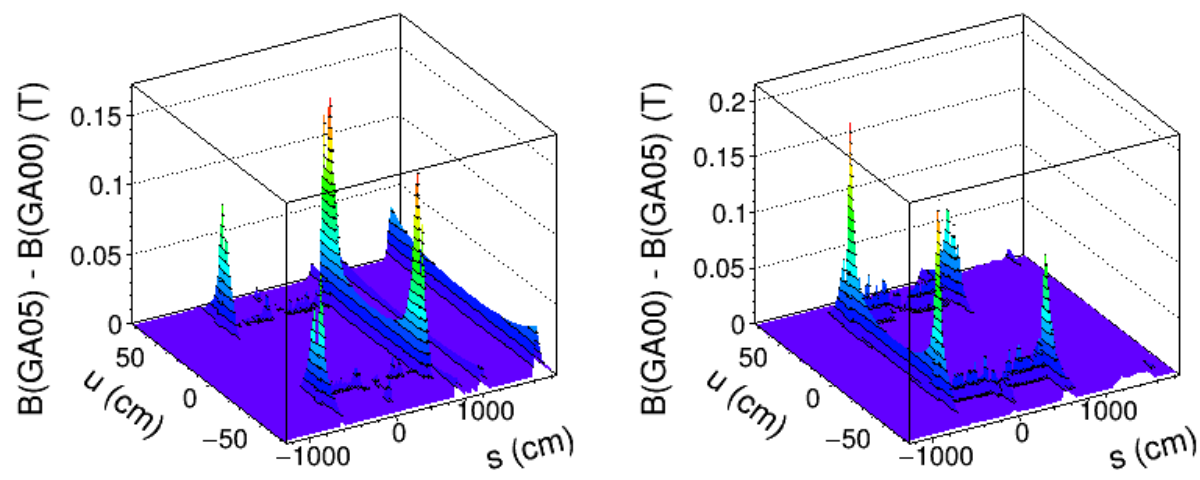

(a) 3D field magnitude differences in the $\mathrm{XZ}$ plane.
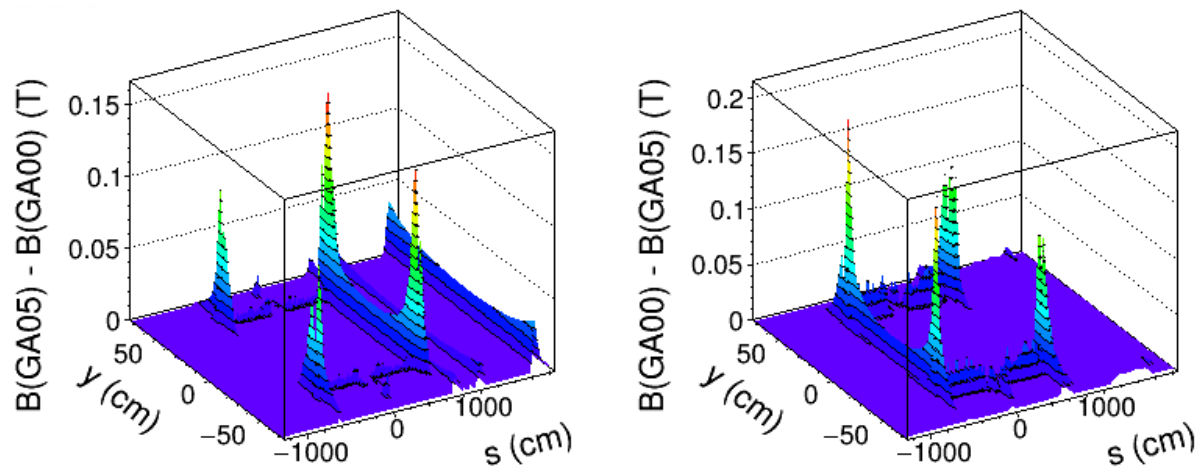

(b) 3D field magnitude differences in the YZ- plane.

Figure 2-5: 3D comparisons between GA05 and GA00 (Helical Test) field maps. The magnetic field difference in T between the two maps is represented in 3D, as function of the path length s (from the entrance of PS through the exit of DS) and the transverse coordinate (u or $y)$ in the local coordinate system. For each figure, the plot on the left represents the positive difference between the GA05 and GA00 fields, while that on the right represents the negative difference between GA05 and GA00.

comparison between field values interpolated with either method at the same points is required. In order to test the interpolation algorithms, $10^{6}$ randomly chosen points in the vacuum volume of DS have been provided. The magnetic field values of these points are genuine ones, since they are calculated by OPERA3D without interpolation. The field values at these points have been calculated using both interpolators, and then compared to the corresponding points calculated with OPERA3D.

Figure 2-6 shows scatter plots of DS grid points differing by more than $10 \mathrm{G}$ with the $10^{6}$ random points calculated by OPERA3D. There are only 5483 points calculated with the tri-quadratic interpolator, out of 1 million, differing by more than $10 \mathrm{G}$ with the genuine ones, while the number of tri-linear interpolated points is almost double. Thus, the quadratic interpolator appears to be 

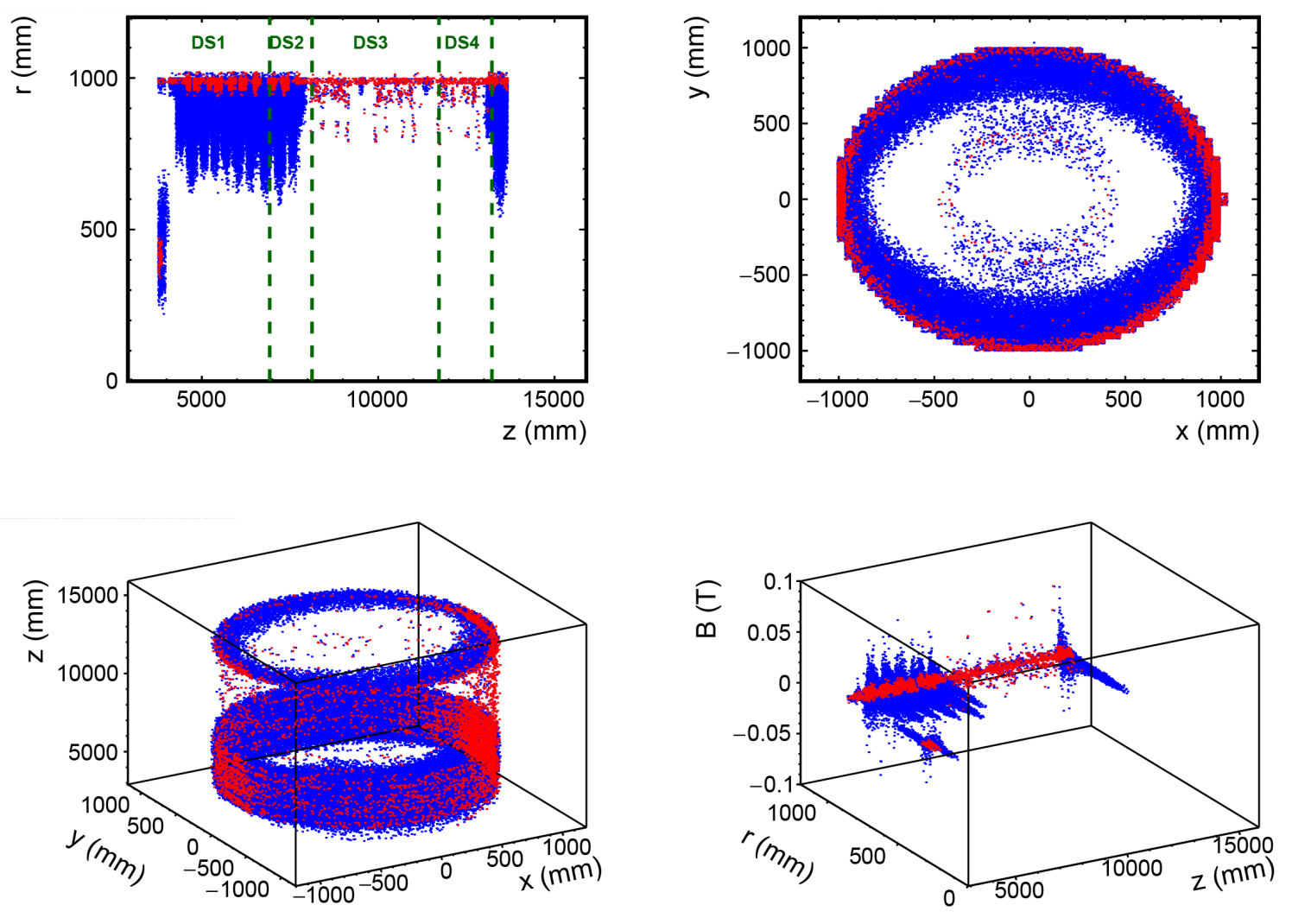

Figure 2-6: Scatter plots of DS grid points where the field magnitude differs by more than $10 \mathrm{G}$ between the $10^{6}$ random points calculated by OPERA3D and the corresponding values calculated in Offline, using tri-linear (blue points) and tri-quadratic (red points) interpolators. The coordinate systems used are $(u, y, s)$ and $(r, \Phi, s)$.

more accurate than the linear, as expected. However, in the Offline software it is preferable to use the linear interpolator, since it is faster. The biggest differences between calculated and interpolated field values in DS are present in the stopping target, tracker and calorimeter regions at large radii, but they are generally small. The test on the interpolator algorithms shows that the most prominent differences (bigger than $100 \mathrm{G}$ ) are close to the coils, thus in a region of no physical interest.

\subsection{Field MAP CALCULATIONS USING MATLAB}

\subsubsection{The SolCalc package}

SolCalc is a software suite that computes and displays magnetic fields generated by a threedimensional solenoid system [Bartoszek et al.(2014)]. Written in MATLAB language [MathWorks $\left.{ }^{\circledR}(2015 b)\right]$, it contains functions that allow to calculate the cartesian components of the magnetic field $\left(B_{x}, B_{y}\right.$ and $\left.B_{z}\right)$ at a given $(X, Y, Z)$ point for a single solenoid positioned and oriented anywhere in space 
or for a solenoid system. The calculation performed by SolCALC is based on the Biot-Savart law [Jackson(1999)], providing the magnetic field created by a current density distribution:

$$
\vec{B}(\vec{r})=\frac{\mu_{0}}{4 \pi} \int \frac{\vec{J}\left(\overrightarrow{r^{\prime}}\right) \times\left(\vec{r}-\overrightarrow{r^{\prime}}\right)}{\left|\vec{r}-\overrightarrow{r^{\prime}}\right|^{3}} d^{3} \overrightarrow{r^{\prime}}
$$

where $\vec{J}$ is the current density, $\vec{r}$ is the point in space where the field is calculated, $\overrightarrow{r^{\prime}}$ is the point where the current density is defined and $\mu_{0}$ is the magnetic permeability of the vacuum. For ideal coils, the calculation involves the computation of two elliptical integrals over two cylinders with the radius equal to the inner and outer radius of the coils, which are subtracted to give the neat contribution of the coil.

Table 2.2: SolCALC input data for computation of the field of PS (coils $1-3$ ) and TSu (coils $4-28$ ). The data corresponds to the Mau9 design. The variables are defined in the text.

\begin{tabular}{|c|c|c|c|c|c|c|c|c|c|c|}
\hline CoIL & $\begin{array}{c}\mathrm{R}_{\text {in }} \\
(\mathrm{mm})\end{array}$ & $\begin{array}{c}\mathrm{R}_{\text {out }} \\
(\mathrm{mm})\end{array}$ & $\begin{array}{c}\mathrm{L} \\
(\mathrm{mm})\end{array}$ & $\begin{array}{c}\mathrm{x}_{0} \\
(\mathrm{~mm})\end{array}$ & $\begin{array}{c}\mathrm{y}_{0} \\
(\mathrm{~mm})\end{array}$ & $\begin{array}{c}\mathrm{z}_{0} \\
(\mathrm{~mm})\end{array}$ & $\begin{array}{c}\alpha \\
\left(^{\circ}\right)\end{array}$ & $\begin{array}{c}\beta \\
\left({ }^{\circ}\right)\end{array}$ & $\begin{array}{l}\gamma \\
\left({ }^{\circ}\right)\end{array}$ & $\begin{array}{c}\text { I } \\
\text { (A) }\end{array}$ \\
\hline 1 & 850 & 946 & 1710 & 3904 & 0 & -6814 & 0 & 0 & 0 & 7866000 \\
\hline 2 & 850 & 914 & 1332 & 3904 & 0 & -5253 & 0 & 0 & 0 & 4084800 \\
\hline 3 & 850 & 914 & 750 & 3904 & 0 & -4092 & 0 & 0 & 0 & 2300000 \\
\hline 4 & 405 & 423 & 172.6 & 3904 & 0 & -3629 & 0 & 0 & 0 & 147050 \\
\hline 5 & 405 & 430.3 & 284.2 & 3904 & 0 & -3379 & 0 & 0 & 0 & 339080 \\
\hline 6 & 405 & 444.9 & 162.4 & 3904 & 0 & -3136.5 & 0 & 0 & 0 & 304480 \\
\hline 7 & 405 & 448.6 & 172.6 & 3904 & 0 & -2929 & 0 & 0 & 0 & 352920 \\
\hline 8 & 405 & 448.6 & 172.6 & 3894 & 0 & -2687 & 0 & -5.7 & 0 & 352920 \\
\hline 9 & 405 & 448.6 & 172.6 & 3864 & 0 & -2447 & 0 & -10.5 & 0 & 352920 \\
\hline 10 & 405 & 463.2 & 172.6 & 3807 & 0 & -2182 & 0 & -15.8 & 0 & 470560 \\
\hline 11 & 405 & 463.2 & 172.6 & 3723 & 0 & -1914 & 0 & -21.3 & 0 & 470560 \\
\hline 12 & 405 & 463.2 & 172.6 & 3613 & 0 & -1656 & 0 & -26.8 & 0 & 470560 \\
\hline 13 & 405 & 463.2 & 172.6 & 3479 & 0 & -1409 & 0 & -32.3 & 0 & 470560 \\
\hline 14 & 405 & 466.8 & 172.6 & 3322 & 0 & -1176 & 0 & -37.8 & 0 & 499970 \\
\hline 15 & 405 & 466.8 & 172.6 & 3143 & 0 & -960 & 0 & -43.3 & 0 & 499970 \\
\hline 16 & 405 & 466.8 & 172.6 & 2944 & 0 & -761 & 0 & -48.7 & 0 & 499970 \\
\hline 17 & 405 & 466.8 & 172.6 & 2728 & 0 & -582 & 0 & -54.2 & 0 & 499970 \\
\hline 18 & 405 & 470.5 & 172.6 & 2495 & 0 & -425 & 0 & -59.7 & 0 & 529380 \\
\hline 19 & 405 & 470.5 & 172.6 & 2248 & 0 & -291 & 0 & -65.2 & 0 & 529380 \\
\hline 20 & 405 & 470.5 & 172.6 & 1990 & 0 & -181 & 0 & -70.7 & 0 & 529380 \\
\hline 21 & 405 & 470.5 & 172.6 & 1722 & 0 & -97 & 0 & -76.2 & 0 & 529380 \\
\hline 22 & 405 & 470.5 & 172.6 & 1457 & 0 & -40 & 0 & -81.5 & 0 & 529380 \\
\hline 23 & 405 & 477.8 & 172.6 & 1217 & 0 & -10 & 0 & -86.3 & 0 & 588200 \\
\hline 24 & 405 & 448.6 & 172.6 & 970 & 0 & 0 & 0 & -90 & 0 & 352920 \\
\hline 25 & 465 & 523.2 & 172.6 & 751.3 & 0 & 0 & 0 & -90 & 0 & 470560 \\
\hline 26 & 465 & 512.2 & 81.2 & 588.3 & 0 & 0 & 0 & -90 & 0 & 179920 \\
\hline 27 & 465 & 519.5 & 172.6 & 425.4 & 0 & 0 & 0 & -90 & 0 & 441150 \\
\hline 28 & 465 & 621.7 & 81.2 & 153.8 & 0 & 0 & 0 & -90 & 0 & 595120 \\
\hline
\end{tabular}


Table 2.3: SolCALC input data for computation of the field of TSd (coils 29-55) and DS (coils 56 - 66). The data corresponds to the Mau9 design. The variables are defined in the text.

\begin{tabular}{|c|c|c|c|c|c|c|c|c|c|c|}
\hline CoIL & $\begin{array}{c}\mathrm{R}_{\text {in }} \\
(\mathrm{mm})\end{array}$ & $\begin{array}{c}\mathrm{R}_{o u t} \\
(\mathrm{~mm})\end{array}$ & $\begin{array}{c}\mathrm{L} \\
(\mathrm{mm})\end{array}$ & $\begin{array}{c}\mathrm{x}_{0} \\
(\mathrm{~mm})\end{array}$ & $\begin{array}{c}\mathrm{y}_{0} \\
(\mathrm{~mm})\end{array}$ & $\begin{array}{c}\mathrm{z}_{0} \\
(\mathrm{~mm})\end{array}$ & $\begin{array}{c}\alpha \\
\left({ }^{\circ}\right)\end{array}$ & $\begin{array}{c}\beta \\
\left(^{\circ}\right)\end{array}$ & $\begin{array}{l}\gamma \\
\left({ }^{\circ}\right)\end{array}$ & $\begin{array}{c}\text { I } \\
\text { (A) }\end{array}$ \\
\hline 29 & 465 & 618.1 & 81.2 & -153.8 & 0 & 0 & 0 & -90 & 0 & 581280 \\
\hline 30 & 465 & 512.2 & 172.6 & -425.4 & 0 & 0 & 0 & -90 & 0 & 382330 \\
\hline 31 & 465 & 515.9 & 81.2 & -588.3 & 0 & 0 & 0 & -90 & 0 & 193760 \\
\hline 32 & 465 & 515.9 & 172.6 & -751.3 & 0 & 0 & 0 & -90 & 0 & 411740 \\
\hline 33 & 405 & 441.3 & 172.6 & -970 & 0 & 0 & 0 & -90 & 0 & 294100 \\
\hline 34 & 405 & 448.6 & 172.6 & -1217 & 0 & 10 & 0 & -86.5 & 0 & 352920 \\
\hline 35 & 405 & 448.6 & 172.6 & -1457 & 0 & 40 & 0 & -81.7 & 0 & 352920 \\
\hline 36 & 405 & 459.5 & 172.6 & -1722 & 0 & 97 & 0 & -76.4 & 0 & 441150 \\
\hline 37 & 405 & 459.5 & 172.6 & -1990 & 0 & 181 & 0 & -70.9 & 0 & 441150 \\
\hline 38 & 405 & 459.5 & 172.6 & -2248 & 0 & 291 & 0 & -65.4 & 0 & 441150 \\
\hline 39 & 405 & 459.5 & 172.6 & -2495 & 0 & 425 & 0 & -59.9 & 0 & 441150 \\
\hline 40 & 405 & 463.2 & 172.6 & -2728 & 0 & 582 & 0 & -54.4 & 0 & 470560 \\
\hline 41 & 405 & 463.2 & 172.6 & -2944 & 0 & 761 & 0 & -48.9 & 0 & 470560 \\
\hline 42 & 405 & 463.2 & 172.6 & -3143 & 0 & 960 & 0 & -43.5 & 0 & 470560 \\
\hline 43 & 405 & 463.2 & 172.6 & -3322 & 0 & 1176 & 0 & -38 & 0 & 470560 \\
\hline 44 & 405 & 463.2 & 172.6 & -3479 & 0 & 1409 & 0 & -32.5 & 0 & 470560 \\
\hline 45 & 405 & 463.2 & 172.6 & -3613 & 0 & 1656 & 0 & -27 & 0 & 470560 \\
\hline 46 & 405 & 463.2 & 172.6 & -3723 & 0 & 1914 & 0 & -21.5 & 0 & 470560 \\
\hline 47 & 405 & 463.2 & 172.6 & -3807 & 0 & 2182 & 0 & -16 & 0 & 470560 \\
\hline 48 & 405 & 455.9 & 172.6 & -3864 & 0 & 2447 & 0 & -10.7 & 0 & 411740 \\
\hline 49 & 405 & 455.9 & 172.6 & -3894 & 0 & 2687 & 0 & -5.9 & 0 & 411740 \\
\hline 50 & 405 & 444.9 & 172.6 & -3904 & 0 & 2929 & 0 & 0 & 0 & 323510 \\
\hline 51 & 405 & 430.3 & 172.6 & -3904 & 0 & 3151.6 & 0 & 0 & 0 & 205870 \\
\hline 52 & 405 & 426.7 & 172.6 & -3904 & 0 & 3339.8 & 0 & 0 & 0 & 176460 \\
\hline 53 & 405 & 419.4 & 172.6 & -3904 & 0 & 3528.1 & 0 & 0 & 0 & 117640 \\
\hline 54 & 405 & 415.7 & 172.6 & -3904 & 0 & 3716.4 & 0 & 0 & 0 & 88230 \\
\hline 55 & 405 & 412.1 & 172.6 & -3904 & 0 & 3904.7 & 0 & 0 & 0 & 58820 \\
\hline 56 & 1050 & 1091 & 419.75 & -3904 & 0 & 3748 & 0 & 0 & 0 & 892644 \\
\hline 57 & 1050 & 1091 & 419.75 & -3904 & 0 & 4173.88 & 0 & 0 & 0 & 892644 \\
\hline 58 & 1050 & 1091 & 419.75 & -3904 & 0 & 4598.88 & 0 & 0 & 0 & 892644 \\
\hline 59 & 1050 & 1091 & 419.75 & -3904 & 0 & 5251.88 & 0 & 0 & 0 & 892644 \\
\hline 60 & 1050 & 1091 & 362.25 & -3904 & 0 & 5880.13 & 0 & 0 & 0 & 770364 \\
\hline 61 & 1050 & 1091 & 362.25 & -3904 & 0 & 6570.13 & 0 & 0 & 0 & 770364 \\
\hline 62 & 1050 & 1091 & 362.25 & -3904 & 0 & 7397.13 & 0 & 0 & 0 & 770364 \\
\hline 63 & 1050 & 1070.5 & 1830 & -3904 & 0 & 8817.75 & 0 & 0 & 0 & 1491816 \\
\hline 64 & 1050 & 1070.5 & 1830 & -3904 & 0 & 10652.8 & 0 & 0 & 0 & 1491816 \\
\hline 65 & 1050 & 1070.5 & 1830 & -3904 & 0 & 12488.2 & 0 & 0 & 0 & 1491816 \\
\hline 66 & 1050 & 1091 & 362.25 & -3904 & 0 & 13642.5 & 0 & 0 & 0 & 770364 \\
\hline
\end{tabular}


SolCALc can also calculate the trajectories of a charged particle through a solenoid system. It takes advantage of the MATLAB's Parallel Computing Toolbox enabling the use of multi-core CPUs which considerably reduces computation times.

The input file (Tables 2.2 and 2.3) for the calculation of the magnetic fields is a list of coordinates and current value, including: inner radius $\left(R_{\text {in }}\right)$, outer radius $\left(R_{\text {out }}\right)$ and length of the coil $(L),\left(\mathrm{x}_{0}\right.$, $\left.y_{0}, z_{0}\right)$ position of the center of the coil, three angles $(\alpha, \beta, \gamma)$ which give the orientation of the coil axis and the total current $(I)$. The angles are defined with respect to the local $(\mathrm{u}, \mathrm{y}, \mathrm{s})$ coordinate system: $\alpha$ is the pitch, i.e. rotation angle about the $\mathrm{u}$ axis, $\beta$ is the yaw, i.e. the rotation angle about the $y$ azis, and $\gamma$ is the roll, i.e. rotation angle about the s axis. All the lengths are in meters, the angles in degree and the current in Amperes.

The SolCALC package has been used in order to create new field maps for PS, TS and DS, applying variations in the alignment of the solenoids or parallel coil displacements. Although only TS misalignments are considered, the field of all solenoids is computed coherently for each variation, in order to include the effect of the fringe fields of the solenoids. The default input file corresponds to Mau9 field maps: they have the same coil geometry, locations and currents. The SolCalc maps do not assume XZ-symmetry, so they are double-sided maps.

\subsubsection{SolCalc validation}

The fist step for the construction of new maps is to compare the SolCalc maps calculated with the default input file with the corresponding Mau9 maps, in order to validate SolCALC results. Figure 2-7 shows the differences of the magnetic field magnitude between SolCalc default maps and the corresponding Mau9 maps. The only differences are in TSu and TSd, at large radii, where the coils are. These may be due to different geometry configurations or different integral computations in the material of the coils. The overall differences between the fields are tiny, of the order of few Gauss in the fiducial volume. Thus, the default field maps calculated with SoLCALC are consistent with the corresponding Mau9 maps.

\subsection{FIELD VARIATIONS}

In order to study the impact of TS field uncertainties on physics parameters of the Mu2e experiment, TSu and TSd field maps have been changed within ad hoc variations. These variations include rotations and parallel coils displacements. There is an enormous amount of degrees of freedom to inspect, thus the cases examined are only indicative. All the examined field variations concern only the field. All the Mu2e geometry regarding the location of materials, including collimators, vacuum bores, cryostats and the coils themselves as geometric objects, is entirely unchanged. Effectively, only the field lines are varied in this study. 

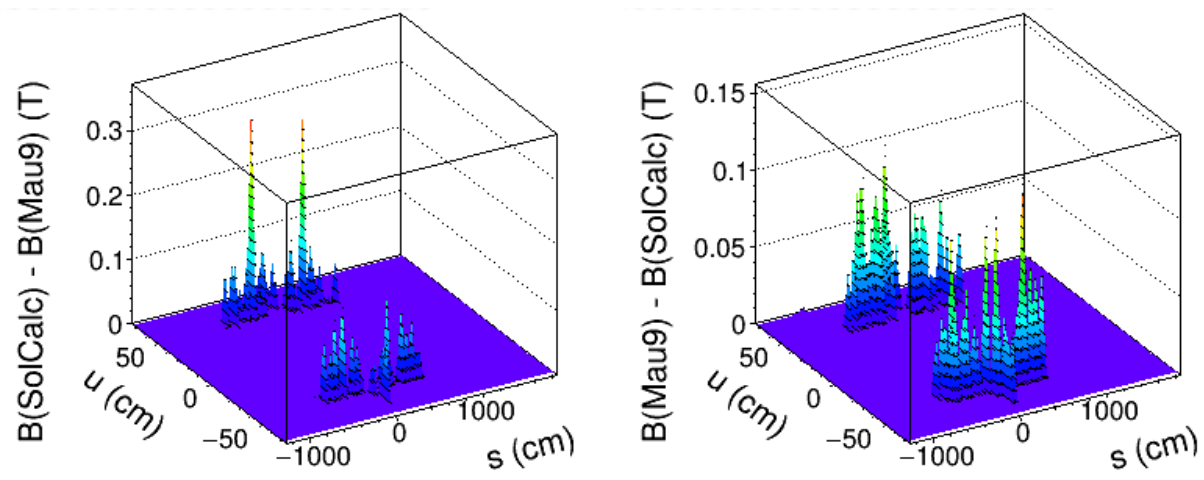

(a) Field magnitude differences in the $\mathrm{XZ}$ plane.
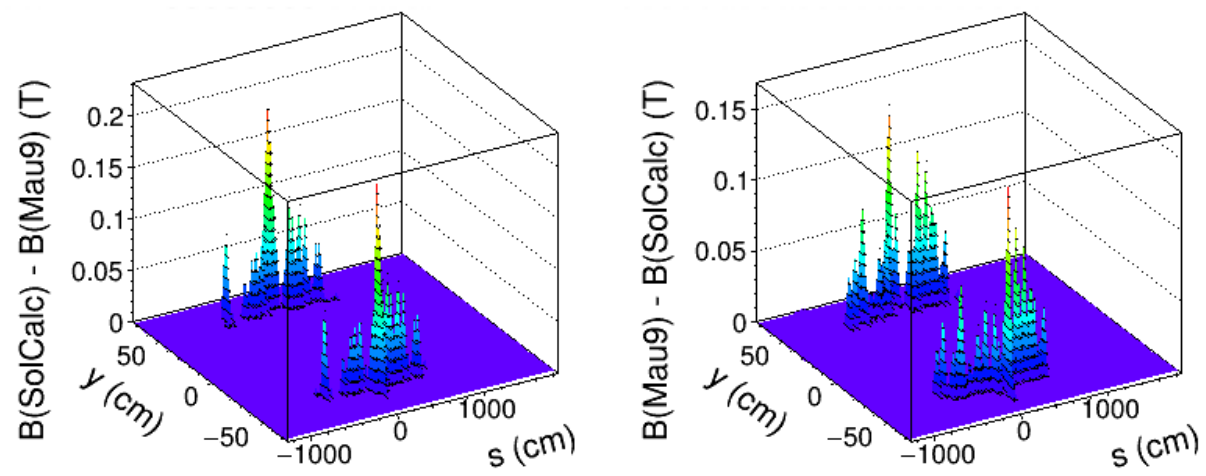

(b) Field magnitude differences in the YZ- plane.

Figure 2-7: Comparisons between SolCalc default maps and Mau9 field maps. The magnetic field difference in T between the two maps is represented in $3 D$, as function of the path length $\mathrm{s}$ (from the entrance of PS through the exit of DS) and the transverse coordinate ( $u$ or $y)$ in the local coordinate system. For each figure, the plot on the left represents the positive difference between the SolCalc and Mau9 fields, while that on the right represents the negative difference between SolCalc and Mau9.

There are two main sets of varied maps: one set is provided by the TD and includes rotations of TS ; the second set has been calculated using SoLCALC.

\subsubsection{Rotations}

The first set of varied maps is constructed on the basis of Mau10 maps, using OPERA3D, without assuming XZ plane symmetry. The rotation is applied only on TS, treated as a rigid body. The solenoid is rotated with respect to the $-\mathrm{Z}$ axis by $0.15^{\circ}$ and $0.50^{\circ}$. Figure $2-8$ shows an exaggerated TS rotation with respect to the $+Z$ axis, that is in the opposite sense of the rotations performed in this study. These two rotations correspond to a maximum coil misalignment at the PS/TSu and TSd/DS boundaries of $10 \mathrm{~mm}$ for $0.15^{\circ}$ rotation, and of $35 \mathrm{~mm}$ for $0.50^{\circ}$ rotation. Studies made by 
the Technical Division have shown that the rotation about the $-\mathrm{Z}$ axis causes the largest deflections of $100 \mathrm{MeV} / \mathrm{c}$ charged particles from their nominal trajectories in the default field. Figure 2-9 shows comparisons between the $0.15^{\circ}$ rotated maps and the corresponding Mau10 default maps. The observed differences between the magnitude of the two calculations are small, up to a few $G$ in the $X Z$ plane and a few tens of $G$ in the $Y Z$ plane, where the rotation takes place. The differences are noticeable at the boundaries between PS and TSu, and TSd and DS.

Table 2.4: Combination of Mau and SolCalc rotated maps with respect to the $Z$ and $X$ axis respectively. TSu and TSd are rotated independently.

\begin{tabular}{l||c|cc}
\hline \hline Maps & Amplitude & TSu & TSd \\
\hline \multirow{2}{*}{ MaU10 } & $0.15^{\circ}$ & $-Z$ & $-Z$ \\
& $0.50^{\circ}$ & $-Z$ & $-Z$ \\
& & & \\
\multirow{3}{*}{ SolCALC (MAU9) } & $0.1^{\circ}$ & $+X$ & $+X$ \\
& $0.1^{\circ}$ & $-X$ & $-X$ \\
& $0.1^{\circ}$ & $+X$ & $-X$ \\
& $0.1^{\circ}$ & $-X$ & $+X$ \\
SolCALC (MAU9) & & & \\
& $1^{\circ}$ & $-X$ & $+X$ \\
& $1^{\circ}$ & $+X$ & $-X$ \\
& $1^{\circ}$ & $-X$ & $-X$ \\
\hline \hline
\end{tabular}

The second set of rotated maps has been created using SolCALC, which corresponds to the Mau 9 default maps. The amount of the rotation is of $1^{\circ}$, corresponding to a maximum coil misalignment at the PS/TSu and TSd/DS boundaries of $50 \mathrm{~mm}$, and $0.1^{\circ}$, corresponding to a maximum coil shift in the extreme coils of $5 \mathrm{~mm}$. For each angle, a combination of 4 rotated sets of maps has been created rotating TSu and TSd independently with respect to $-\mathrm{X}$ or $+\mathrm{X}$ axis: this kind of rotations keeps TS3 fixed, since this is the TS region most tightly constrained by the mechanical support and collimator infrastructure, thus making misalignments in that region very unlikely. Table 2.4 summarizes the rotations of TS designs considered in this study.

Figure 2-10 illustrates one of the 4 possible rotations for $1^{\circ}$ : TSu and TSd are rotated in the $+X$ direction. The field magnitude difference between this case and the SolCalc default one is plotted in Figure 2-11: also in this case the differences are noticeable at PS/TSu and TSd/DS boundaries. The observed differences between the magnitude of the two calculations are small, up to a few tens of $\mathrm{G}$ in the $\mathrm{XZ}$ plane and a few hundreds of $\mathrm{G}$ in the $\mathrm{YZ}$ plane, where the rotation takes place. In this case, the field differences are one order of magnitude greater than the differences in the case of $0.15^{\circ}$ rotation about the $-\mathrm{Z}$ axis. The misalignments corresponding to $0.50^{\circ} \mathrm{TS}$ rotation about the $-\mathrm{Z}$ axis (Mau10) and $1^{\circ}$ TS rotation about the $\mathrm{X}$ axis (SolCalc) are very unlikely to happen with the 
present engineering systems for big volume alignment. These are merely exhibition cases, to make sure the effects are noticeable, and then the magnitude of the misalignments is reduced to more realistic examples.

\subsubsection{Parallel coil displacements}

Using the SolCALC software, a parallel coil displacement has been introduced in TSu and TSd. The coils from 14 to 18 (in TSu, see Table 2.2) and from 39 to 43 (in TSd, see Table 2.3) have been shifted with respect to $+\mathrm{Y}$ or $-\mathrm{Y}$ axis, for a total combination of 4 sets of maps (Table 2.5). Figure 2-12 shows one of these cases.

Table 2.5: Combination of SolCalc shifted coil maps with respect to the $Y$ axis.

\begin{tabular}{l||c|cc}
\hline \hline Maps & Amplitude & TSu & TSd \\
\hline \multirow{4}{*}{ SolCALC (MaU9) } & $20 \mathrm{~mm}$ & $+Y$ & $+Y$ \\
& $20 \mathrm{~mm}$ & $-Y$ & $-Y$ \\
& $20 \mathrm{~mm}$ & $+Y$ & $-Y$ \\
& $20 \mathrm{~mm}$ & $-Y$ & $+Y$ \\
\hline \hline
\end{tabular}

Figure 2-13 shows the comparison between SolCalc default maps and SolCalc parallel coil displacement with respect to $+Y$ axis. The field magnitude differences are of the order of a few $\mathrm{G}$ in the $\mathrm{XZ}$ plane and of a few tens of $\mathrm{G}$ in the $\mathrm{YZ}$ plane. The misalignments corresponding to parallel coil displacements of $20 \mathrm{~mm}$ are very unlikely to happen with the present engineering systems for big volume alignment. 


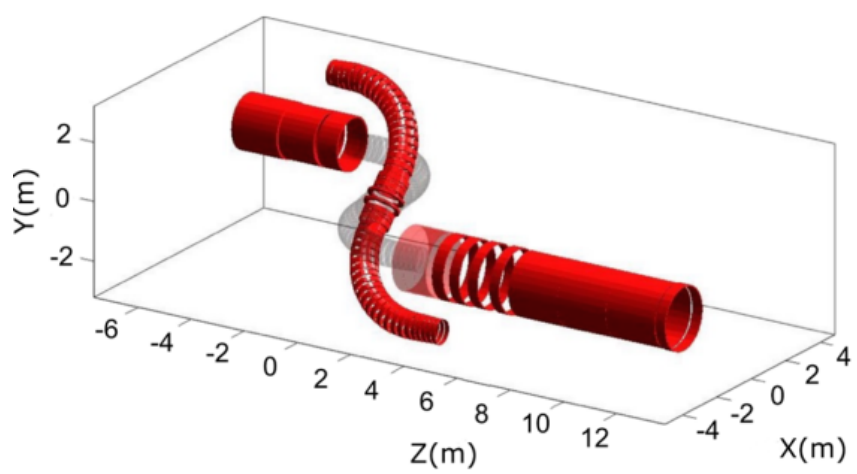

Figure 2-8: Illustration of a rotation of TS with respect to $+Z$ axis. The effect of the rotation are exaggerated.
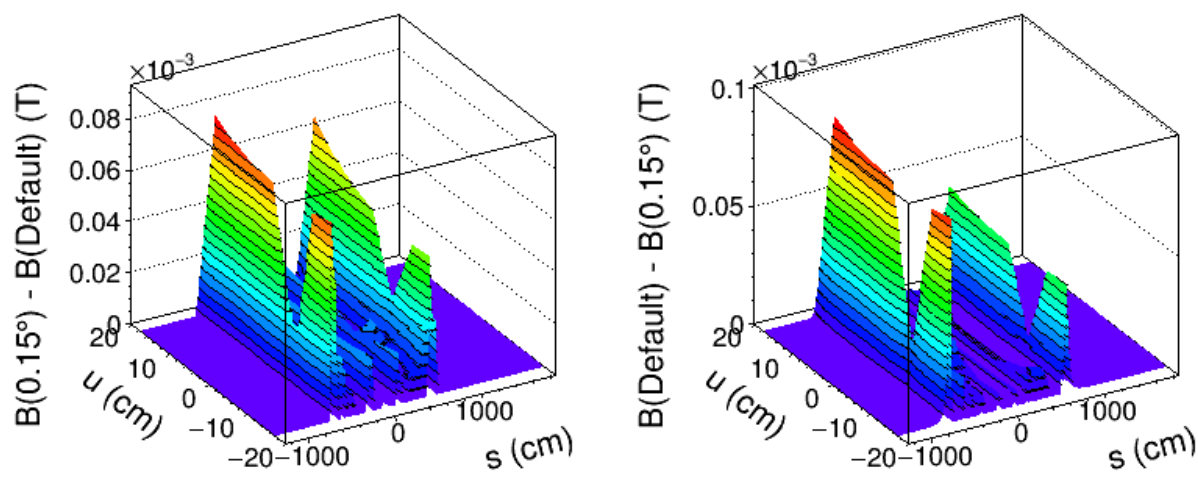

(a) Field magnitude differences in the $\mathrm{XZ}$ plane.
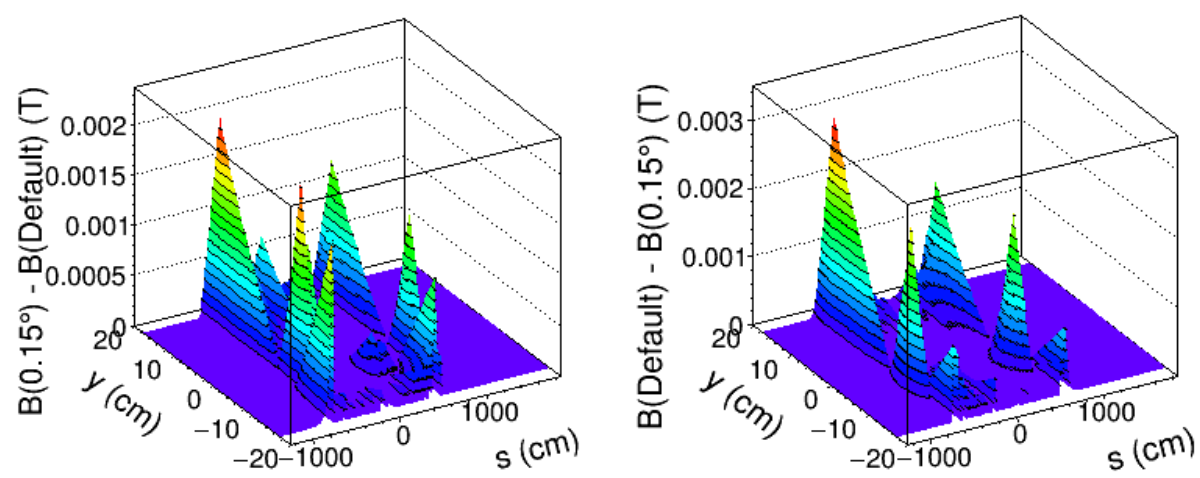

(b) Field magnitude differences in the $\mathrm{YZ}$ plane.

Figure 2-9: Comparisons between Mau10 default maps and Mau10 rotated field maps by $0.15^{\circ}$ with respect to the $-Z$ axis. The field magnitude difference in $T$ between the two maps is represented in $3 D$, as function of the path length $\mathrm{s}$ (from the entrance of PS through the exit of DS) and the transverse coordinate ( $u$ or $y$ ) in the local coordinate system. For each figure, the plot on the left represents the positive difference between the Mau10 default and Mau10 rotated fields, while that on the right represents the negative difference between Mau10 default and rotated. 


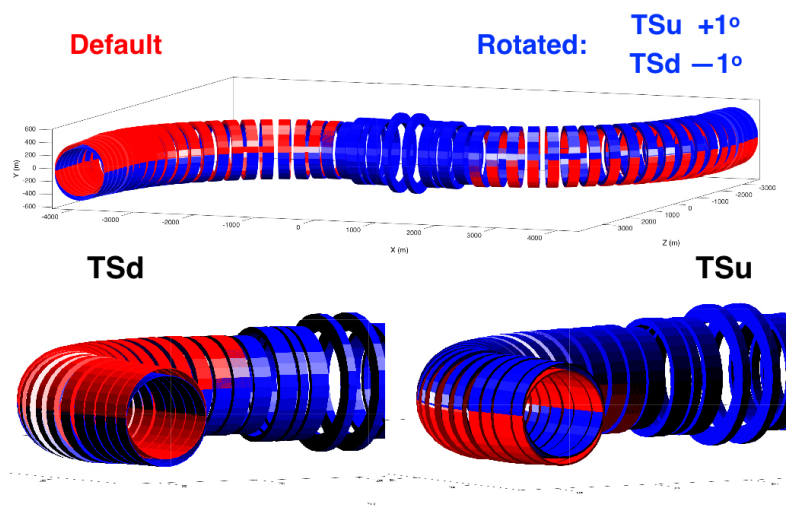

Figure 2-10: Rotation of SolCalc field maps by $1^{\circ}$. Both TSu and TSd are rotated about $+X$ axis. The red coils correspond to default SolCalc field maps, while the blue ones are the rotated coils.
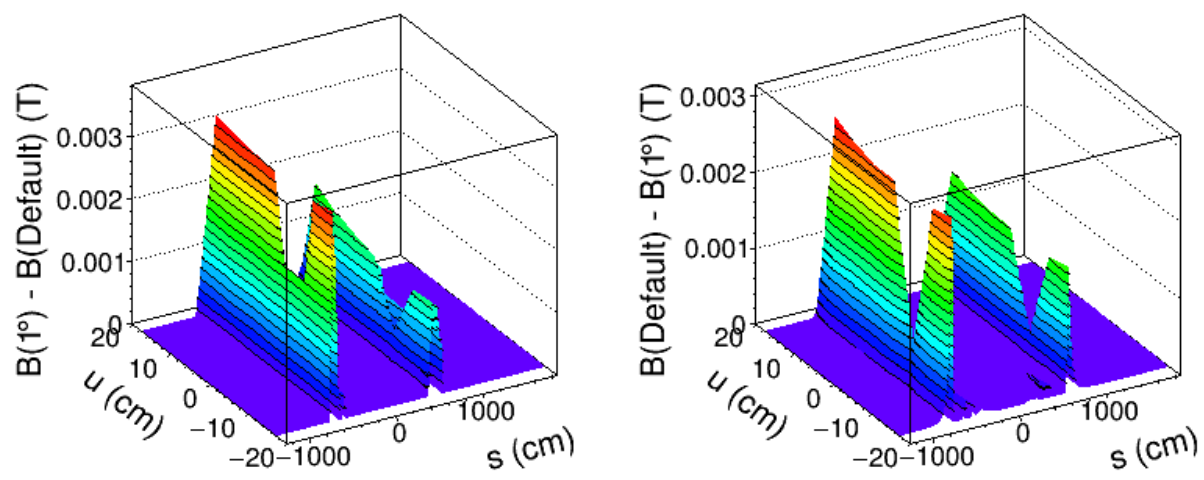

(a) 3D field magnitude differences in the $\mathrm{XZ}$ plane.
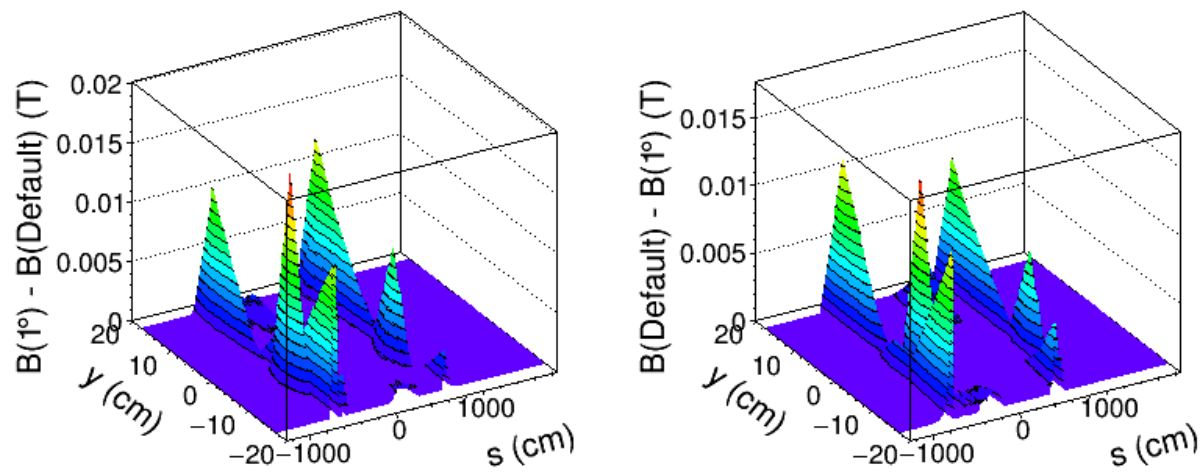

(b) 3D field magnitude differences in the $\mathrm{YZ}$ plane.

Figure 2-11: Comparisons between SolCalc default maps and SolCalc rotated field maps by $1^{\circ}$ with respect to the $+X$ axis for both TSu and TSd. The field magnitude difference in T between the two maps is represented in $3 D$, as function of the path length $\mathrm{s}$ (from the entrance of PS through the exit of DS) and the transverse coordinate (u or $y$ ) in the local coordinate system. For each figure, the plot on the left represents the positive difference between the default and rotated fields, while that on the right represents the negative difference between default and rotated maps. 


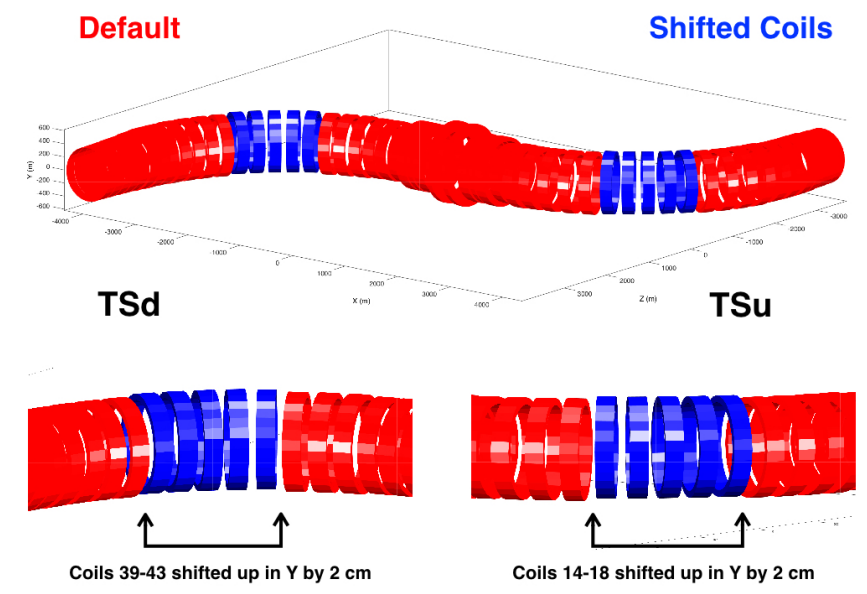

Figure 2-12: Parallel coil displacements in SolCalc maps. The blue coils are shifted up with respect to the Y axis by 20 $\mathrm{mm}$. The red coils represent the default SolCalc configuration.
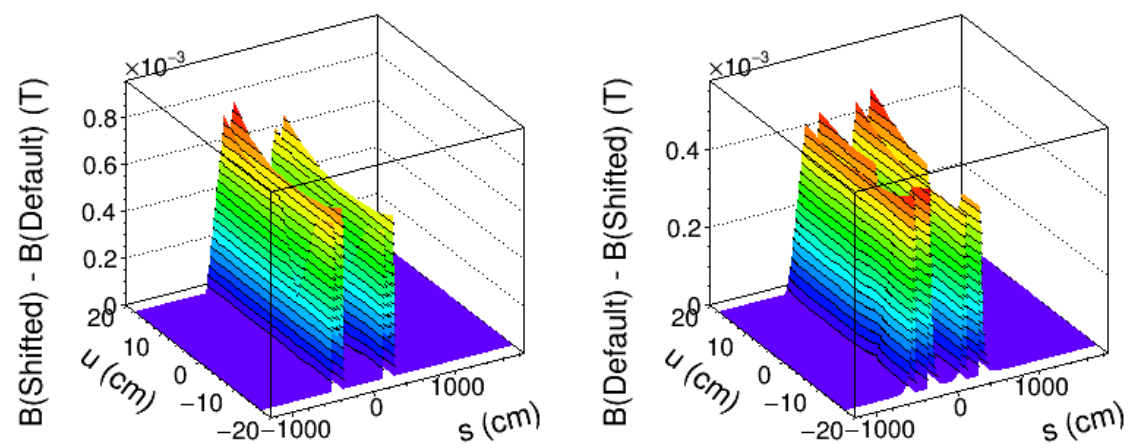

(a) 3D field magnitude differences in the $\mathrm{XZ}$ plane.
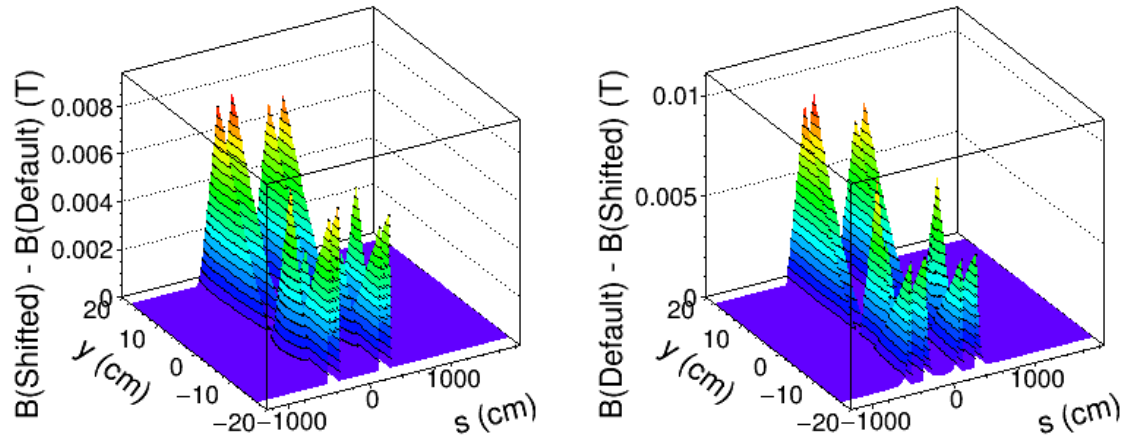

(b) 3D field magnitude differences in the $\mathrm{YZ}$ plane.

Figure 2-13: $3 D$ comparisons between SolCalc default maps and SolCalc with shifted coil by $20 \mathrm{~mm}$ about the $Y$ axis. Both TSu and TSd coils are shifted up. The field magnitude difference in T between the two maps is represented in $3 D$, as function of the path length $\mathrm{s}$ (from the entrance of PS through the exit of DS) and the transverse coordinate ( $u$ or $y$ ) in the local coordinate system. For each figure, the plot on the left represents the positive difference between the default and shifted fields, while that on the right represents the negative difference between default and shifted maps. 


\section{CHAPTER 3}

\section{MUON AND PION STOPPING RATE STUD-}

\section{IES}

TS misalignments can affect the space and time distributions of the particles arriving at the stopping target, thus changing the electron yields from muon capture and pion absorption. Such are the CE and DIO electrons and the electrons from RPC and RMC that can be detected in the detector (see subsection 1.1.3). These changes alter the sensitivity of the experiment and therefore have to be studied to determine tolerance limits for such misalignments, within which the sensitivity does not change significantly.

\subsection{MUON STOPPING TARGET}

The muon capture target is a central component of the Mu2e experiment (see Figure 3-1). Interactions in the stopping target cause energy loss and the capture of the beamline muons after they enter the DS. The muons form muonic atoms at the capture target and then they can potentially undergo neutrinoless conversion to electrons. The capture target design goal is to maximize the number of captured muons while minimizing the amount of material traversed by conversion electrons that enter the acceptance of the downstream detector.

The target for the first run of the Mu2e experiment will be pure aluminum. There are several motivations for choosing aluminum, that include: conversion rate, lifetime, conversion electron energy and related backgrounds. For low $\mathrm{Z}$ nuclei, the sensitivity to electron conversion is roughly proportional to $Z$, therefore $Z$ should be maximized, but the muon capture rate scales approximately with $Z^{4}$, therefore $Z$ should not be too large. The muon capture rate benefits from large $Z$ up to selenium or antimony, so it should be chosen large but not exceeding the value corresponding to those elements. For aluminum, the electron conversion energy is $104.96 \mathrm{MeV}$. The lifetime of 
muonic aluminum (864 ns), is relatively long, enabling the use of the delay of $700 \mathrm{~ns}$ for the muon beam flash to die out before taking data. This reduces the number of background events directly related to the muon beam occurring during the detector live gate time when the conversion electron process takes place. The aluminum target can also suppress a potential background coming from radiative muon capture, via the reaction:

$$
\mu^{-}+{ }_{Z}^{A} X_{N} \rightarrow_{Z-1}^{A} Y_{N+1}^{*}+v_{\mu}+\gamma
$$

In the case of $\mathrm{Al}$ nucleus and of $\mathrm{Mg}$ daughter nucleus, the maximum photon energy, corresponding to zero neutrino momentum, is set by:

$$
E_{\gamma, M A X}=m_{\mu}+\left|E_{b}\right|-E_{\text {recoil }}-\Delta M
$$

where $\left|E_{b}\right|$ is the binding energy of a muon on $\mathrm{Al}(0.47 \mathrm{MeV}), E_{\text {recoil }}$ is the recoil of the $\mathrm{Mg}$ daughter nucleus $(0.21 \mathrm{MeV})$ and $\Delta M$ is the nuclear mass difference of $\mathrm{Mg}$ and $\mathrm{Al}(3.11 \mathrm{MeV})$. The photon can convert into an electron-positron pair of (nearly) equal momenta, from which only the electron can fake a signal. So, this electron would have a maximum momentum of $p_{e, M A X} \simeq \frac{E_{\gamma, M A X}}{2}$. For the muon mass of $105.66 \mathrm{MeV}$, the maximum kinetic energy the photon can acquire is $101.9 \mathrm{MeV}$, thus the electron can only take up to half of this. When the mother nucleus is ${ }_{13}^{27} \mathrm{Al}_{14}$ and the daughter nucleus is ${ }_{12}^{27} \mathrm{Mg}_{15}^{*}$, i.e. unstable magnesium, which is less bound and thus heavier than $\mathrm{Al}$, the $p_{e} \simeq 51 \mathrm{MeV} / \mathrm{c}$, which is roughly $2 \mathrm{MeV} / \mathrm{c}$ lower than the DIO endpoint.

The original design of the muon capture target consisted of 17 aluminum foils, ranging between $Z=5471 \mathrm{~mm}$ and $\mathrm{Z}=6271 \mathrm{~mm}$ in the Mu2e coordinate system. The location of the muon capture target relative to other elements in the DS is shown in Figure 3-1. Each layer of the muon capture target was separated from adjacent layers by $50 \mathrm{~mm}$. The radius of each layer was decreasing in the downstream direction, beginning with $83 \mathrm{~mm}$ and ending at $65 \mathrm{~mm}$. The thickness of each layer was the same for all layers and equal to $200 \mu \mathrm{m}$. The density of aluminum is $2.70 \mathrm{~g} / \mathrm{cm}^{2}$ and therefore the total mass of the muon capture target was $159.4 \mathrm{~g}$. This design is such that incoming muons interact with enough mass to be stopped in the target volume and simultaneously each layer is thin enough to minimize the energy loss in conversion electron such that they pass through the tracker and are properly reconstructed. The decrease in layers's radius reduces the risk that scattered particles can stop in the following foils.

The new baseline design of the muon capture target consists of 34 aluminum foils, ranging between $Z=5871 \mathrm{~mm}$ and $Z=6671 \mathrm{~mm}$ in the Mu2e coordinate system. With respect to the old location shown in Figure 3-1, the new location is shifted $400 \mathrm{~mm}$ downstream, but retaining the same total length of $800 \mathrm{~mm}$. Therefore, each layer is now separated from adjacent layers by 24 $\mathrm{mm}$. The radius of each layer is now constant and equal to $75 \mathrm{~mm}$. The thickness of each layer is 


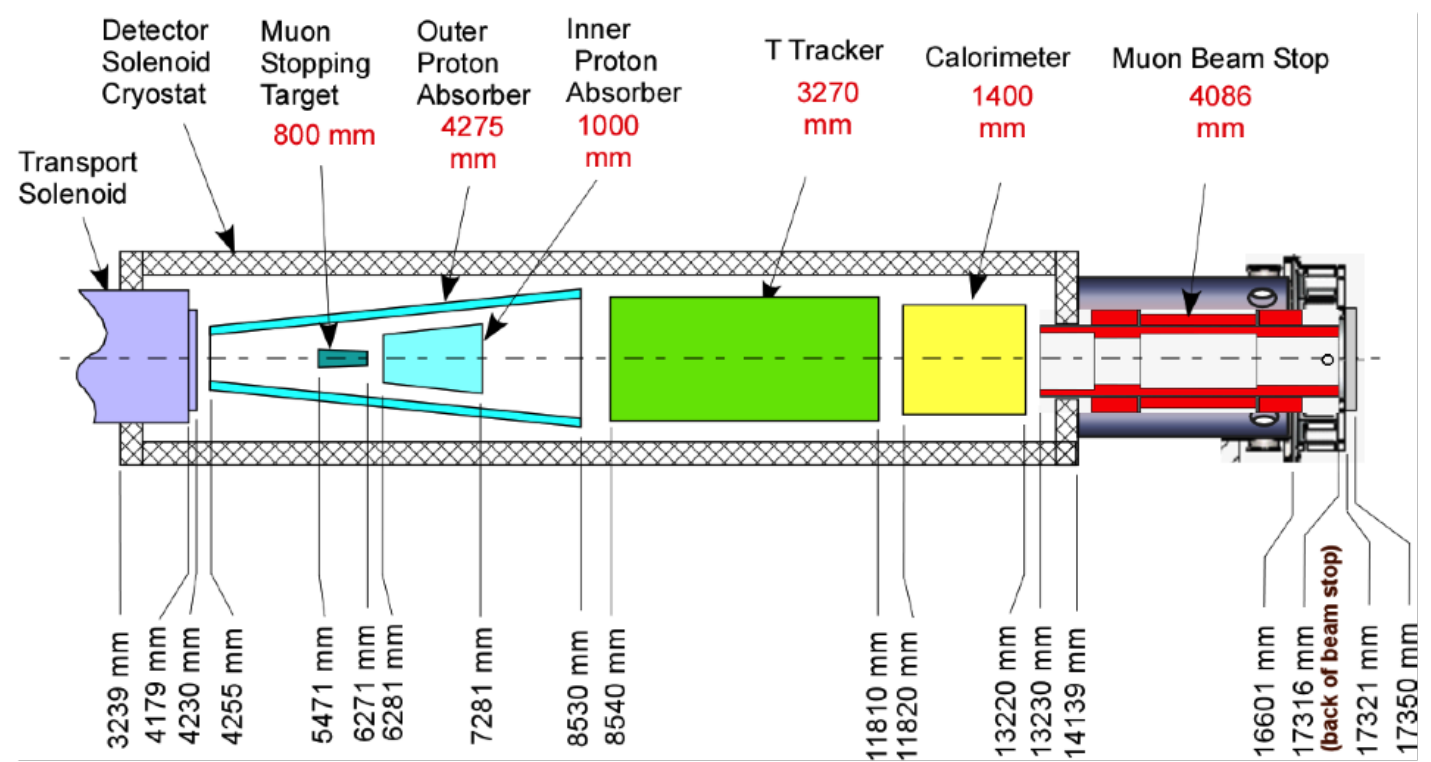

Figure 3-1: Cross section view showing locations of components within the Detector Solenoid. The muon capture target is located between $\mathrm{Z}=471 \mathrm{~mm}$ and $\mathrm{Z}=6271 \mathrm{~mm}$, in the old target design. The Transport Solenoid downstream absorber (TSdA) is the small disk at Z coordinate $\sim 4230 \mathrm{~mm}$. The Outer Proton Absorber $(O P A)$ is illustrated in light blue between $Z=4255 \mathrm{~mm}$ and $Z=8530 \mathrm{~mm}$. Note that the muon capture target and the Inner Proton Absorber (IPA) are surrounded by the OPA.

the same for all layers and equal to $100 \mu \mathrm{m}$. The density of aluminum is $2.70 \mathrm{~g} / \mathrm{cm}^{2}$ and therefore the total mass of the muon capture target is $162 \mathrm{~g}$. Simulation studies have shown that the new design provides better signal sensitivity than the previous one. The new design is the one used in the present study.

The muon capture target is supported by a structure consisting of three wires attached to each layer such that the layer is firmly suspended and centered on the incoming muon beam. The material chosen for the wires is tungsten, since simulations have shown that tungsten has negligible effect on the energy straggling of conversion electrons. In fact, because of the diffuse nature of the muon beam, a significant number of muons can strike the structure supporting the capture target, producing DIO electrons at large radius where the acceptance for reconstruction in the detector is high. Because the endpoint energy of the DIO spectrum decreases for higher Z materials, and because the lifetime of the muons in muonic atoms decreases for higher $\mathrm{Z}$ materials, the support must be constructed from a high $\mathrm{Z}$ material, such as tungsten. Figure 3-2 shows the layers of the muon capture target as well as the tungsten support structure.

\subsection{StOPPED PARTiCle SIMUlations}

A study of the effects of field uncertainties on $\mu^{-}$and $\pi^{-}$stopping rates on the aluminum target has been made. To compute the muon and pion stopping rates described below, a single-stage simulation is performed. This simulation merges stage 1, 2 and 3 together since the simulation 


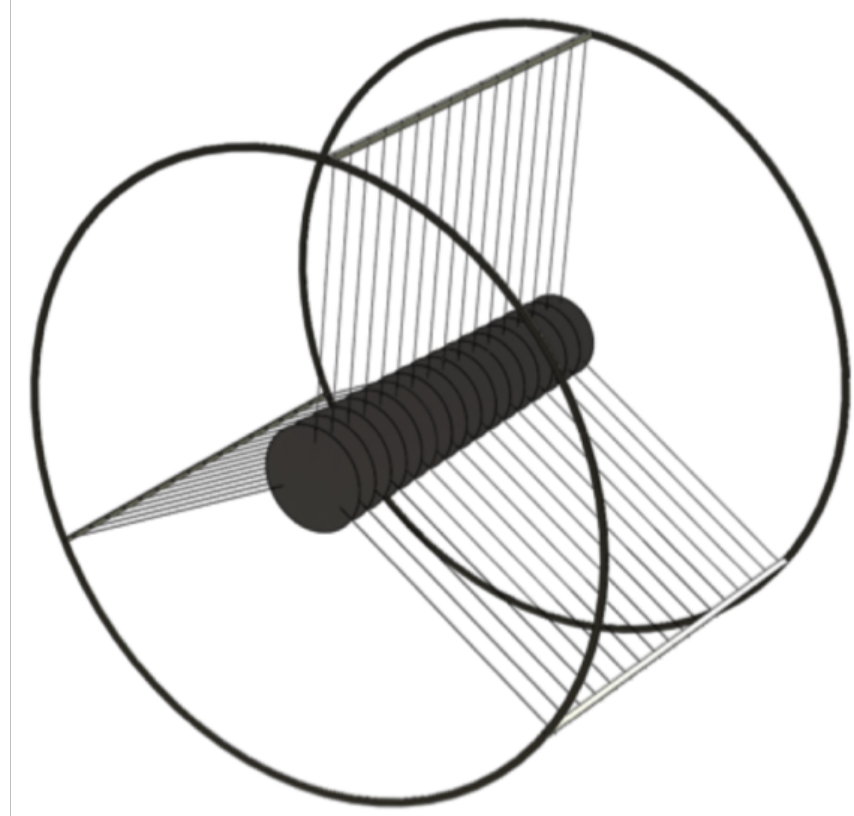

Figure 3-2: Illustration of the old design of the muon capture target composed of 17 aluminum foils and its mechanical support frame. The new capture target design uses the same support frame outlook, but for 34 instead of 17 foils.

is required only up to stage 3 and thus it does not need high statistics for stage 4 (where many particles are lost to limited acceptance and reconstruction efficiency). It begins with $8 \mathrm{GeV}$ protons interacting in the production target and ends at the capture target in DS. The protons are modeled as a delta-function in the time domain with an arrival time at the production target of $\mathrm{t}=0$. The protontungsten scattering process, which produces all particles used subsequently in the simulation (scattered protons, knocked-out neutrons, antiprotons, deuterons, pions, kaons, heavier mesons, etc) is a very detailed multi-process cross section model, able to provide the correct particle production fractions (and thus the correct secondary beam admixture) that theory alone cannot. It is based on combinations of data fits and theoretical calculations. It is worth to note that this model is not in GEANT4. It has been developed specifically for the needs of this experiment as a separate library loaded by the framework. The simulation tracks all produced particles through TS until the upstream portion of the detector solenoid vacuum, and records muon and pion stops in the aluminum capture target. The location and arrival time of muons and pions in capture target are saved.

Figure 3-3 illustrates where the muons are captured in the stopping target using nominal values of the field (Mau10 maps are used), as function of $\mathrm{Z}$ and $\mathrm{r}$, where $\mathrm{Z}$ is the longitudinal Mu2e coordinate along the DS axis and $\mathrm{r}$ is the radial distance from the DS axis. The polar distribution shows that muon rates decrease going through the stopping layers, since muons are captured in the previous layers, but also decrease with increasing $r$, because the muon beam is focused along 
the axis.

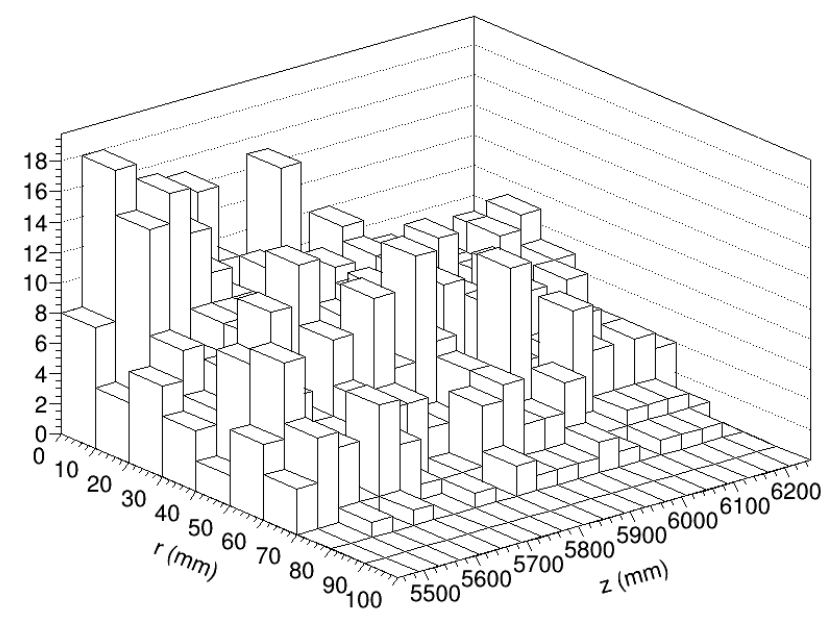

Figure 3-3: Polar distribution of captured muons in the stopping target, as function of $r$ and $\mathrm{Z}$ in mm. Mau10 maps are used for this simulation. The number of captured muons decreases with $r$ and $Z$.

The simulation uses the full Mu2e solenoid system including all collimators, the production solenoid, transport solenoid, detector solenoid, and the latest magnetic-field maps. Parameters for both simulations are described in Table $3.1: 10^{6}$ protons on target (POT) are simulated for muon rates, $10^{7}$ POT for pion rates. For the pion stopping rate simulation 10 times more POT are needed because the muon capture cross section, of atomic origin, is higher than the pion absorption cross section, of nuclear origin. In the muon stopping rate simulation, muons are allowed to decay, whereas in the pion stopping rate simulation the pions are not allowed to decay (i.e. the lifetime is set to infinity), in order to save the statistics through the stopping target.

Table 3.1: Proton beam parameters considered in this study. The simulated events are expressed as function of number of protons on targets (POT).

\begin{tabular}{c||cc}
\hline \hline & Kinetic Energy & Simulated Events \\
\hline$\mu^{-}$ & $8 \mathrm{GeV}$ & $10^{6}$ POT \\
$\pi^{-}$ & $8 \mathrm{GeV}$ & $10^{7}$ POT \\
\hline \hline
\end{tabular}

The effect of PS/TS and DS/TS misalignments, TS rotations and shifted coils on the number of $\mu^{-}$and $\pi^{-}$stops in the $\mathrm{Al}$ is studied using the SolCalc rotated and shifted maps and the Mau10 rotated maps. Both simulations for $\mu^{-}$and $\pi^{-}$are run changing the field maps in the geometry file read by the FHiCL. The samples are statistically independent. 


\subsection{Results}

The muon stopping rate $R_{\mu}$ is defined as the number of captured muons in the stopping target divided by the number of protons hitting the production target. The definition of stopping rate would be the same for the pions, if the pion decay were not turned off in the single-stage simulation. The pion decays are therefore accounted for after the simulation in an approximate way, by using the average pion arrival time $\langle t\rangle$ at the stopping target and the pion life time $\tau=26$ ns:

$$
R_{\pi}=\frac{N_{\pi}}{N_{P O T}} e^{-\langle t\rangle / \tau}
$$

where $R_{\pi}$ is the pion rate, $N_{\pi}$ is number of stopped pions and $N_{P O T}$ is the number of protons on target.

The fractional yield differences derived from varied fields are evaluated using the following formula:

$$
\alpha=\frac{N_{\text {default }}-N_{\text {varied }}}{N_{\text {default }}}, \quad \delta \alpha=\sqrt{\frac{N_{\text {varied }}\left(N_{\text {default }}+N_{\text {varied }}\right)}{N_{\text {default }}^{3}}}
$$

where $N$ is the number of stopped particles. $\alpha$ allows to evaluate how much the rates scale if misalignments occur. This measure is fundamental for the Mu2e success: in fact, the sensitivity of the experiment is scaled on the number of captured muons, while stopped pions in the muon capture target are source of background.

\subsubsection{Rotations}

Table 3.2 shows the muon stopping rates for the default and the rotated maps. On the average, 1 - 2 muons are captured at the $\mathrm{Al}$ target for every 1000 protons hitting the production target. Table 3.3 shows the pion stopping rates for the same maps: the pion yields $\left(N_{\pi}\right)$ and the average arrival pion time are also shown. Up to about 100 pions are absorbed by the $\mathrm{Al}$ target for every billion of protons hitting the production target.

Table 3.4 shows the fractional yield differences for all the rotated maps, for both $\mu^{-}$and $\pi^{-}$ stopping rates. As expected, the biggest differences are for rotations of $0.50^{\circ}$, which corresponds to misalignments of $30 \mathrm{~mm}$ between PS/TS and TS/DS, and of $1^{\circ}$, which corresponds to misalignments of $50 \mathrm{~mm}$ between the same solenoids.

Figure 3-4 and Figure 3-5 illustrate the position and time distributions for stopped $\mu^{-}$using Mau10 rotated maps by $0.15^{\circ}$ and $0.50^{\circ}$, respectively. Figure 3-6 and Figure 3-7 illustrate the position and time distributions for stopped $\pi^{-}$using Mau10 rotated maps by $0.15^{\circ}$ and $0.50^{\circ}$, respectively. Because of the tiny rotation, the shapes are almost the same and the drop in statistics very small.

Figure 3-8 to Figure 3-23 show the position and time distributions for stopped $\mu^{-}$and $\pi^{-}$using 
Table 3.2: Muon stopping rates for rotated maps.

\begin{tabular}{|c|c|c|}
\hline MAPS & $N_{\mu}$ & $R_{\mu}\left(\times 10^{-3}\right)$ \\
\hline SolCalc (Mau9) default & 1862 & $1.86 \pm 0.04$ \\
\hline MaU10 DEFAULT & 1833 & $1.83 \pm 0.04$ \\
\hline MaU10 $0.15^{\circ}$ wrt $-Z$ TSu, $0.15^{\circ}$ wrt $-Z$ TSd & 1852 & $1.85 \pm 0.04$ \\
\hline MaU10 $0.50^{\circ}$ wrt $-Z$ TSu, $0.50^{\circ}$ wrt $-Z$ TSd & 1775 & $1.78 \pm 0.04$ \\
\hline SolCalc (MaU9) $0.1^{\circ}$ wrt $+X$ TSu, $0.1^{\circ}$ wrt $-X$ TSd & 1868 & $1.87 \pm 0.04$ \\
\hline SolCalc (MaU9) $0.1^{\circ}$ wrt $-X$ TSu, $0.1^{\circ}$ wrt $+X$ TSd & 1815 & $1.81 \pm 0.04$ \\
\hline SolCalc (Mau9) $0.1^{\circ} \mathrm{wrt}+X \mathrm{TSu}, 0.1^{\circ} \mathrm{wrt}+X \mathrm{TSd}$ & 1921 & $1.92 \pm 0.04$ \\
\hline SolCalc (Mau9) $0.1^{\circ}$ wrt $-X$ TSu, $0.1^{\circ}$ wrt $-X$ TSd & 1819 & $1.81 \pm 0.04$ \\
\hline SolCalc (MaU9) $1^{\circ}$ wrt $+X$ TSu, $1^{\circ}$ wrt $-X$ TSd & 1774 & $1.77 \pm 0.04$ \\
\hline SolCalc (Mau9) $1^{\circ}$ wrt $-X \mathrm{TSu}, 1^{\circ}$ wrt $+X$ TSd & 1467 & $1.47 \pm 0.04$ \\
\hline SolCalc (Mau9) $1^{\circ}$ wrt $+X$ TSu, $1^{\circ}$ wrt $+X$ TSd & 1472 & $1.47 \pm 0.04$ \\
\hline SolCalc (Mau9) $1^{\circ}$ wrt $-X$ TSu, $1^{\circ}$ wrt $-X$ TSd & 1020 & $1.02 \pm 0.03$ \\
\hline
\end{tabular}

Table 3.3: Pion stopping rates for rotated maps.

\begin{tabular}{|c|c|c|c|}
\hline MAPS & $N_{\pi}$ & $\langle t\rangle(\mathrm{ns})$ & $R_{\pi}\left(\times 10^{-8}\right)$ \\
\hline SolCalc (Mau9) default & 24762 & 266.3 & $8.82 \pm 0.06$ \\
\hline Mau10 Default & 23428 & 266.1 & $8.41 \pm 0.05$ \\
\hline MAU10 $0.15^{\circ}$ wrt $-Z$ TSu, $0.15^{\circ}$ wrt $-Z$ TSd & 24229 & 265.8 & $8.80 \pm 0.06$ \\
\hline MaU10 $0.50^{\circ}$ wrt $-Z$ TSu, $0.50^{\circ}$ wrt $-Z$ TSd & 20194 & 276.1 & $4.94 \pm 0.03$ \\
\hline SolCalc (Mau9) $0.1^{\circ} \mathrm{wrt}+X \mathrm{TSu}, 0.1^{\circ} \mathrm{wrt}-X \mathrm{TSd}$ & 24960 & 268.0 & $8.33 \pm 0.05$ \\
\hline SolCalc (Mau9) $0.1^{\circ}$ wrt $-X$ TSu, $0.1^{\circ}$ wrt $+X$ TSd & 24627 & 264.4 & $9.44 \pm 0.06$ \\
\hline SolCalc (MaU9) $0.1^{\circ} \mathrm{wrt}+X \mathrm{TSu}, 0.1^{\circ} \mathrm{wrt}+X$ TSd & 25166 & 266.0 & $9.07 \pm 0.06$ \\
\hline SolCalc (Mau9) $0.1^{\circ} \mathrm{wrt}-X \mathrm{TSu}, 0.1^{\circ} \mathrm{wrt}-X \mathrm{TSd}$ & 23375 & 266.5 & $8.26 \pm 0.05$ \\
\hline SolCalc (Mau9) $1^{\circ}$ wrt $+X$ TSu, $1^{\circ}$ wrt $-X$ TSd & 26039 & 261.1 & $11.33 \pm 0.07$ \\
\hline SolCalc (Mau9) $1^{\circ}$ wrt $-X$ TSu, $1^{\circ}$ wrt $+X$ TSd & 18490 & 264.2 & $7.14 \pm 0.05$ \\
\hline SolCalc (MaU9) $1^{\circ}$ wrt $+X$ TSu, $1^{\circ}$ wrt $+X$ TSd & 16701 & 285.7 & $2.82 \pm 0.02$ \\
\hline SolCalc (MaU9) $1^{\circ}$ wrt $-X$ TSu, $1^{\circ}$ wrt $-X$ TSd & 13839 & 253.3 & $8.13 \pm 0.07$ \\
\hline
\end{tabular}

the SolCalc rotated maps of $1^{\circ}$ and $0.1^{\circ}$. As the field misalignment is increased, a difference in the shape appears between the varied maps and the nominal one. This is evident in Figure 3-9 and Figure 3-13, which correspond to the biggest rotations analyzed in this study of $1^{\circ}$ in the SolCalc maps. The shape differs particularly in the $Y$ distribution: in fact, the effects of the rotations are predominant in the $y$ coordinate, since the rotation takes place in the $Y Z$ plane. The $z$ distributions reveal the foil structure of the muon capture target. The $\mathrm{X}$ distribution in Figure 3-13 shows clearly a drop in the statistics observed for $\pi^{-}$distribution: a fractional yield difference of the $45 \%$ is 
Table 3.4: Fractional Yield Differences for $\mu^{-}$and $\pi^{-}$for the rotated maps. The $\alpha$ and its error are expressed in percentage.

\begin{tabular}{|c|c|c|}
\hline & $\alpha_{\mu}(\%)$ & $\alpha_{\pi}(\%)$ \\
\hline MAU10 $0.15^{\circ}$ wrt $-Z$ TSu, $0.15^{\circ}$ wrt $-Z$ TSd & $-1.04 \pm 3.33$ & $-3.42 \pm 0.95$ \\
\hline MAU10 $0.50^{\circ}$ wrt $-Z \mathrm{TSu}, 0.50^{\circ}$ wrt $-Z$ TSd & $3.16 \pm 3.22$ & $13.80 \pm 0.83$ \\
\hline SolCALC (MAU9) $0.1^{\circ} \mathrm{wrt}+X \mathrm{TSu}, 0.1^{\circ} \mathrm{wrt}-X$ TSd & $-0.32 \pm 3.29$ & $-0.80 \pm 0.90$ \\
\hline SolCALC (MAU9) $0.1^{\circ}$ wrt $-X$ TSu, $0.1^{\circ}$ wrt $+X$ TSd & $2.52 \pm 3.22$ & $0.55 \pm 0.90$ \\
\hline SolCALC (MAU9) $0.1^{\circ} \mathrm{wrt}+X$ TSu, $0.1^{\circ} \mathrm{wrt}+X$ TSd & $-3.17 \pm 3.36$ & $-1.63 \pm 0.91$ \\
\hline SolCalc (Mau9) $0.1^{\circ}$ wrt $-X$ TSu, $0.1^{\circ}$ wrt $-X$ TSd & $2.31 \pm 3.22$ & $5.60 \pm 0.86$ \\
\hline SolCalc (Mau9) $1^{\circ}$ wrt $+X$ TSu, $1^{\circ}$ wrt $-X$ TSd & $4.73 \pm 3.16$ & $-5.16 \pm 0.93$ \\
\hline SolCalc (Mau9) $1^{\circ}$ wrt $-X$ TSu, $1^{\circ}$ wrt $+X$ TSd & $21.21 \pm 2.75$ & $25.33 \pm 0.73$ \\
\hline SolCalc (Mau9) $1^{\circ}$ wrt $+X$ TSu, $1^{\circ}$ wrt $+X$ TSd & $20.94 \pm 2.76$ & $32.55 \pm 0.68$ \\
\hline SolCalc (Mau9) $1^{\circ}$ wrt $-X$ TSu, $1^{\circ}$ wrt $-X$ TSd & $45.22 \pm 2.13$ & $44.11 \pm 5.93$ \\
\hline
\end{tabular}

observed, as reported in Table 3.4.

The results show that for exaggerated misalignments (maximum coil shifts $30-50 \mathrm{~mm}$ ) there are big effects both in the shape and in the integral of both stopped muon and stopped pion distributions. The effects are more dramatic in the vertical $(Y)$ dimension, where the coils are displaced by the rotation. For TSd misalignments in the direction above the $\mathrm{XZ}$ plane, there is an increase in the total number of particles stopped at the target. Large effects are also observed in the slope of the $\mathrm{Z}$ coordinate distributions: the slope becomes steeper when the misalignment is stronger. For realistic misalignments (maximum coil shifts $5-10 \mathrm{~mm}$ ), no significant effects are observed in the stopped muon distributions. Some significant effects are still observed in the stopped pion distributions, particularly for cases where TSd is displaced in the direction above the $\mathrm{XZ}$ plane. In all of these realistic cases, the uncertainty introduced to the estimates of RPC background from the total numbers of stopped pions is of the same order with the uncertainty reported in the TDR (Table 1.1). 

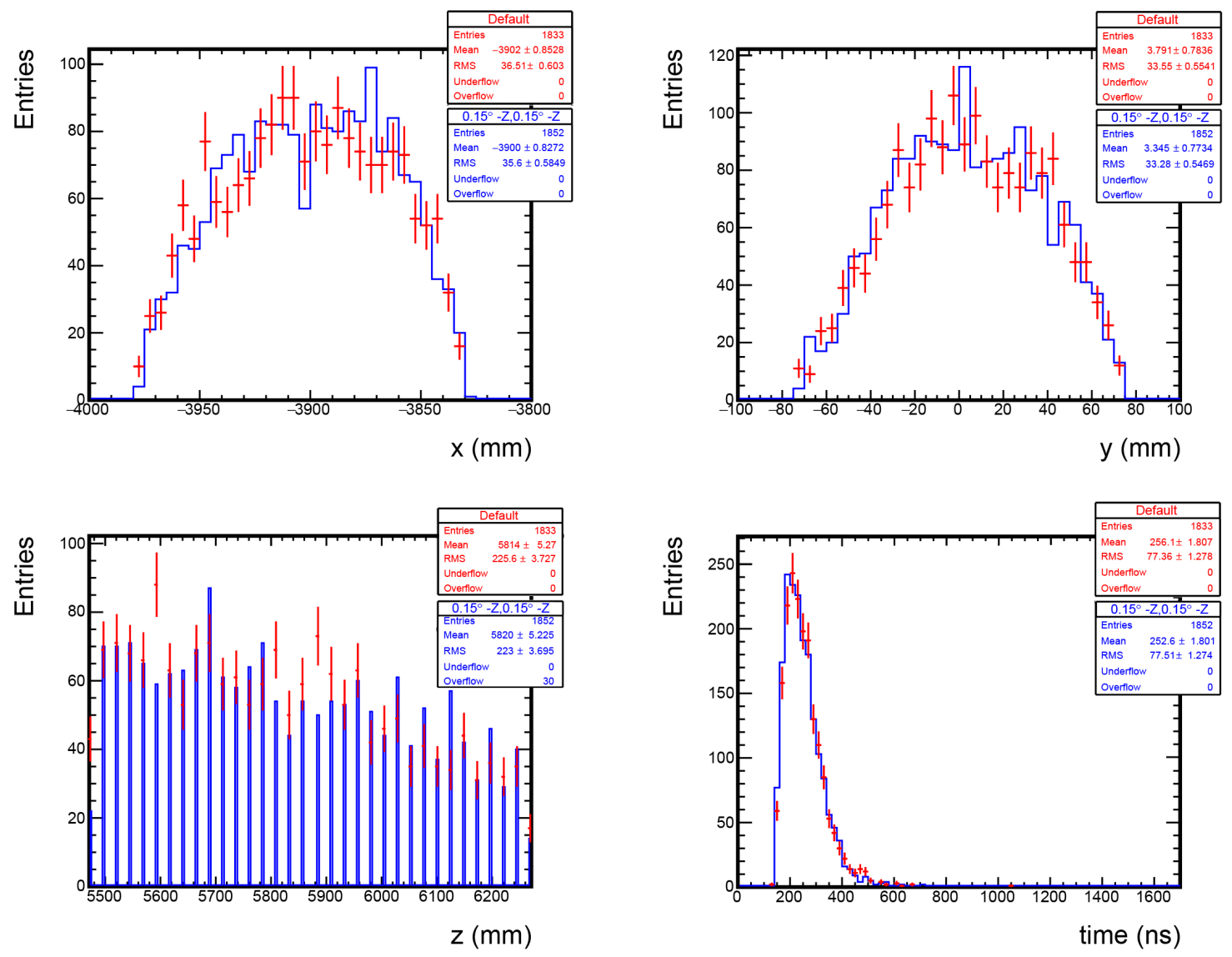

Figure 3-4: $X, Y, Z$ and time distributions for stopped muons in Mu2e coordinates, using Mau10 nominal (red) and rotated maps of $0.15^{\circ}$ (blue) with respect to the $-\mathrm{Z}$ axis. 

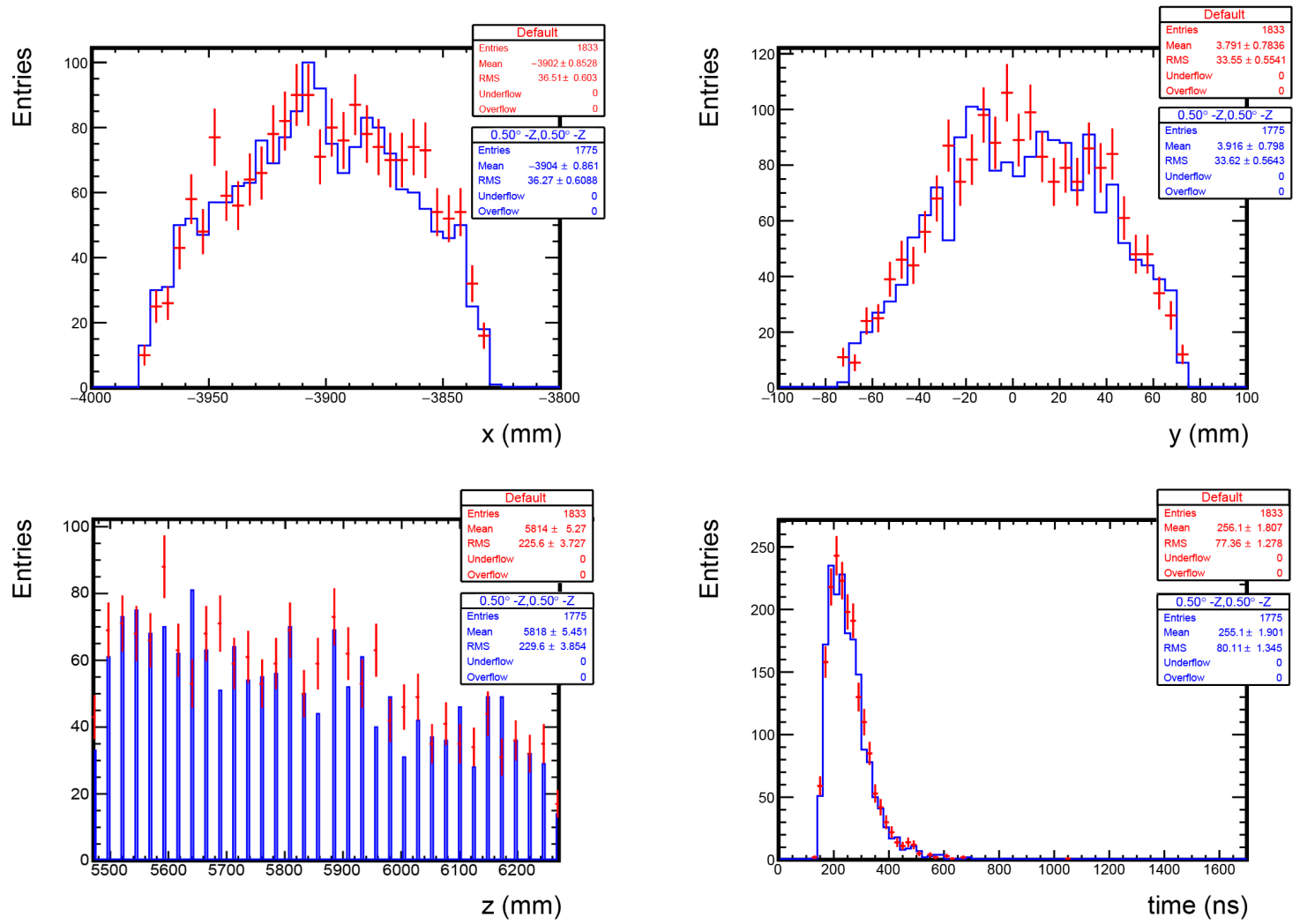

Figure 3-5: $X, Y, Z$ and time distributions for stopped muons in Mu2e coordinates, using Mau10 nominal (red) and rotated maps of $0.50^{\circ}$ (blue) with respect to the $-\mathrm{Z}$ axis. 

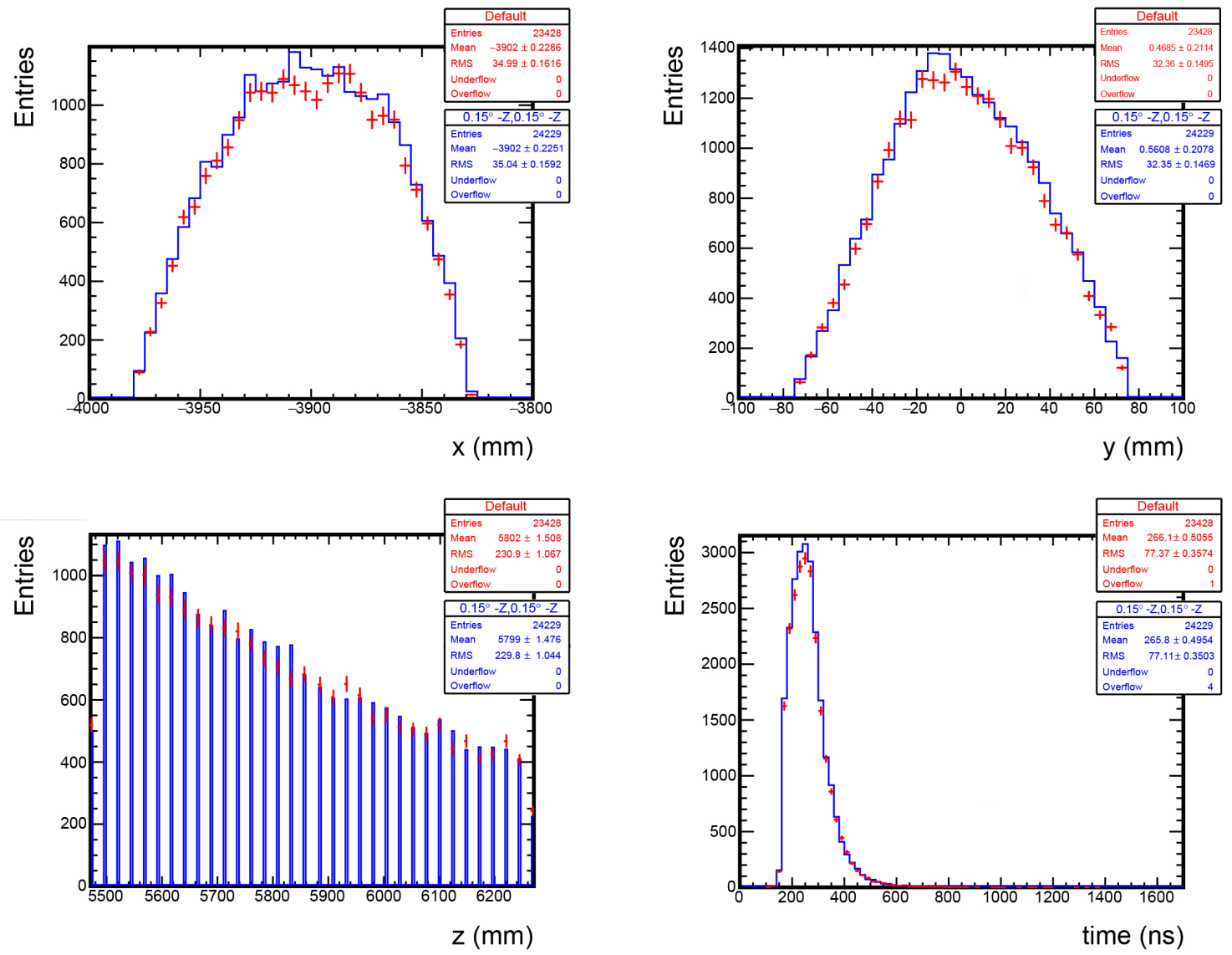

Figure 3-6: $X, Y, Z$ and time distributions for stopped pions in Mu2e coordinates, using Mau10 nominal (red) and rotated maps of $0.15^{\circ}$ (blue) with respect to the $-\mathrm{Z}$ axis. 

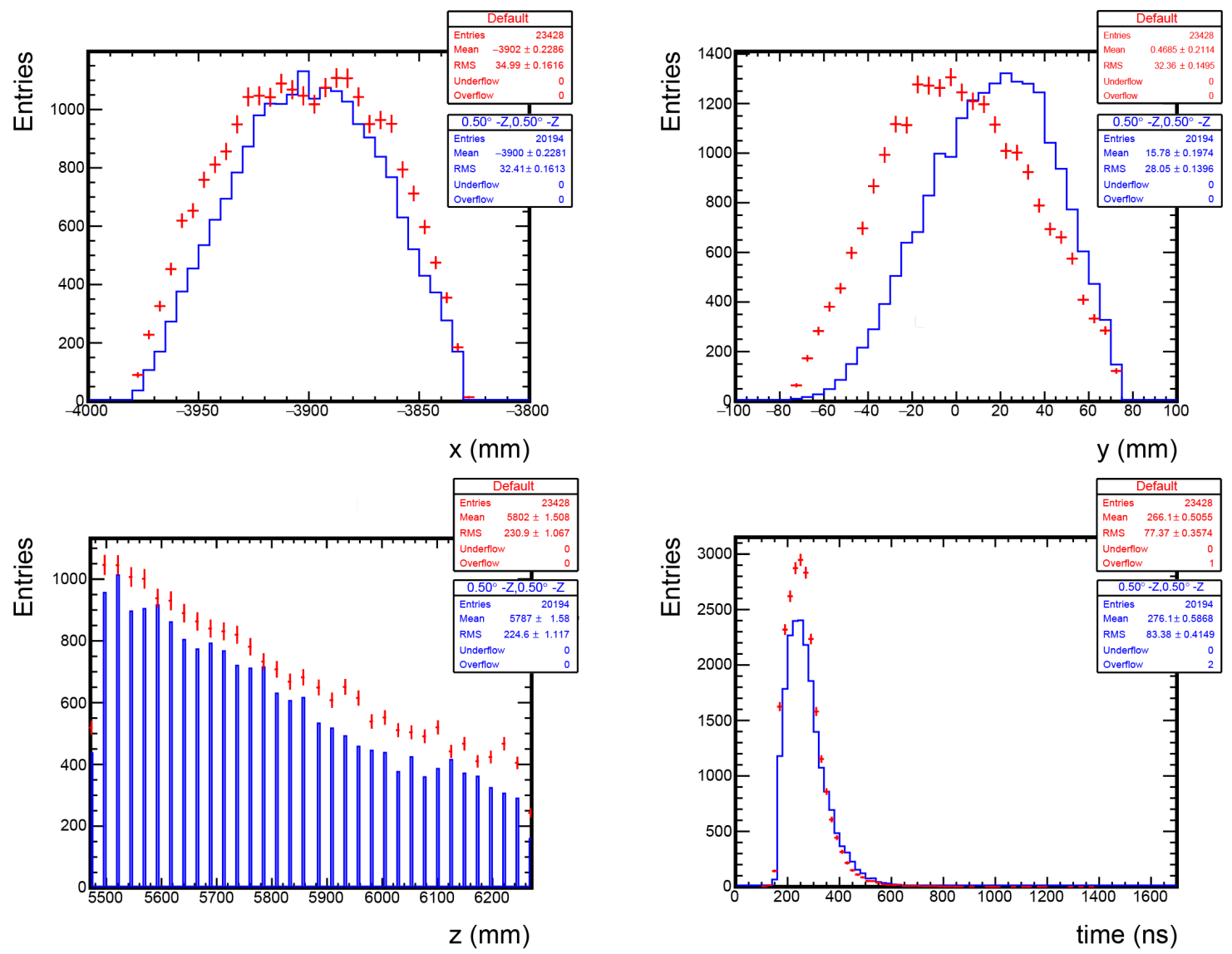

Figure 3-7: $X, Y, Z$ and time distributions for stopped pions in Mu2e coordinates, using Mau10 nominal (red) and rotated maps of $0.50^{\circ}$ (blue) with respect to the $-\mathrm{Z}$ axis. 

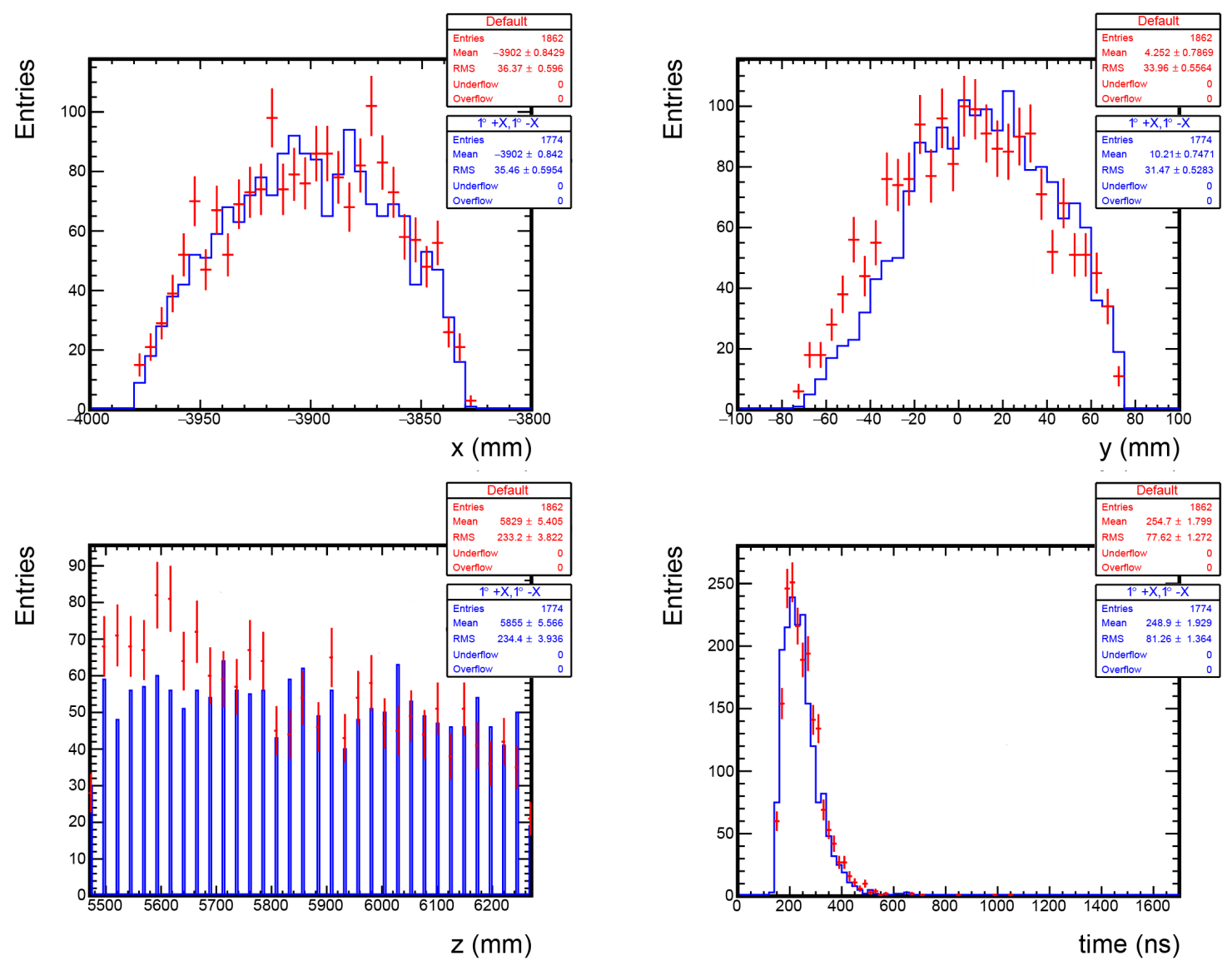

Figure 3-8: Stopped muon spatial and time distributions in Mu2e coordinate system, using SolCalc nominal field (red) and the field corresponding to a rotation of $1^{\circ}$ about $+X$ of TSu and $1^{\circ}$ about $-X$ of TSd (blue). 

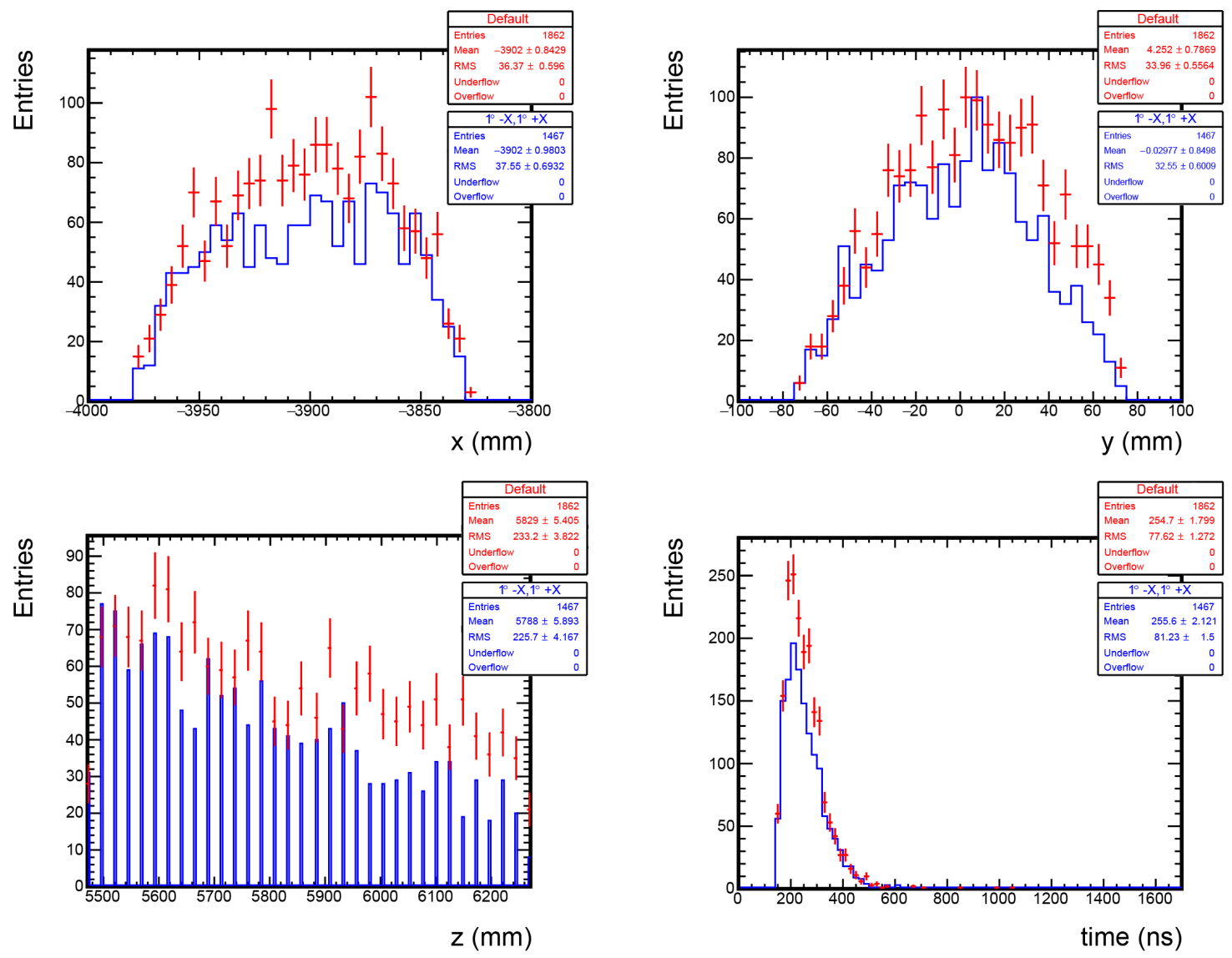

Figure 3-9: Stopped muon spatial and time distributions in Mu2e coordinate system, using SolCalc nominal field (red) and the field corresponding to a rotation of $1^{\circ}$ about $-X$ of TSu and $1^{\circ}$ about $+X$ of TSd (blue). 

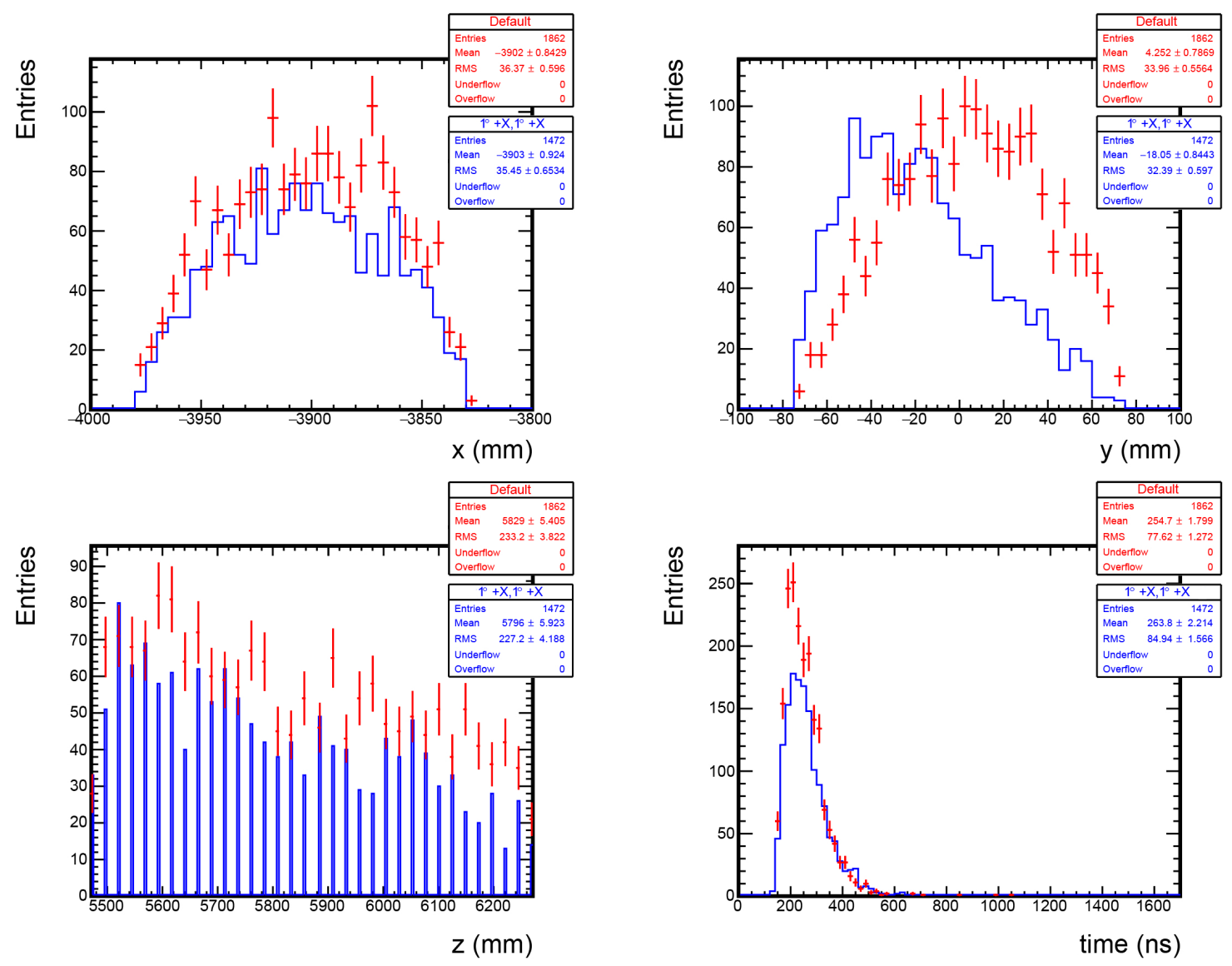

Figure 3-10: Stopped muon spatial and time distributions in Mu2e coordinate system, using SolCalc nominal field (red) and the field corresponding to a rotation of $1^{\circ}$ about $+X$ of TSu and $1^{\circ}$ about $+X$ of TSd (blue). 

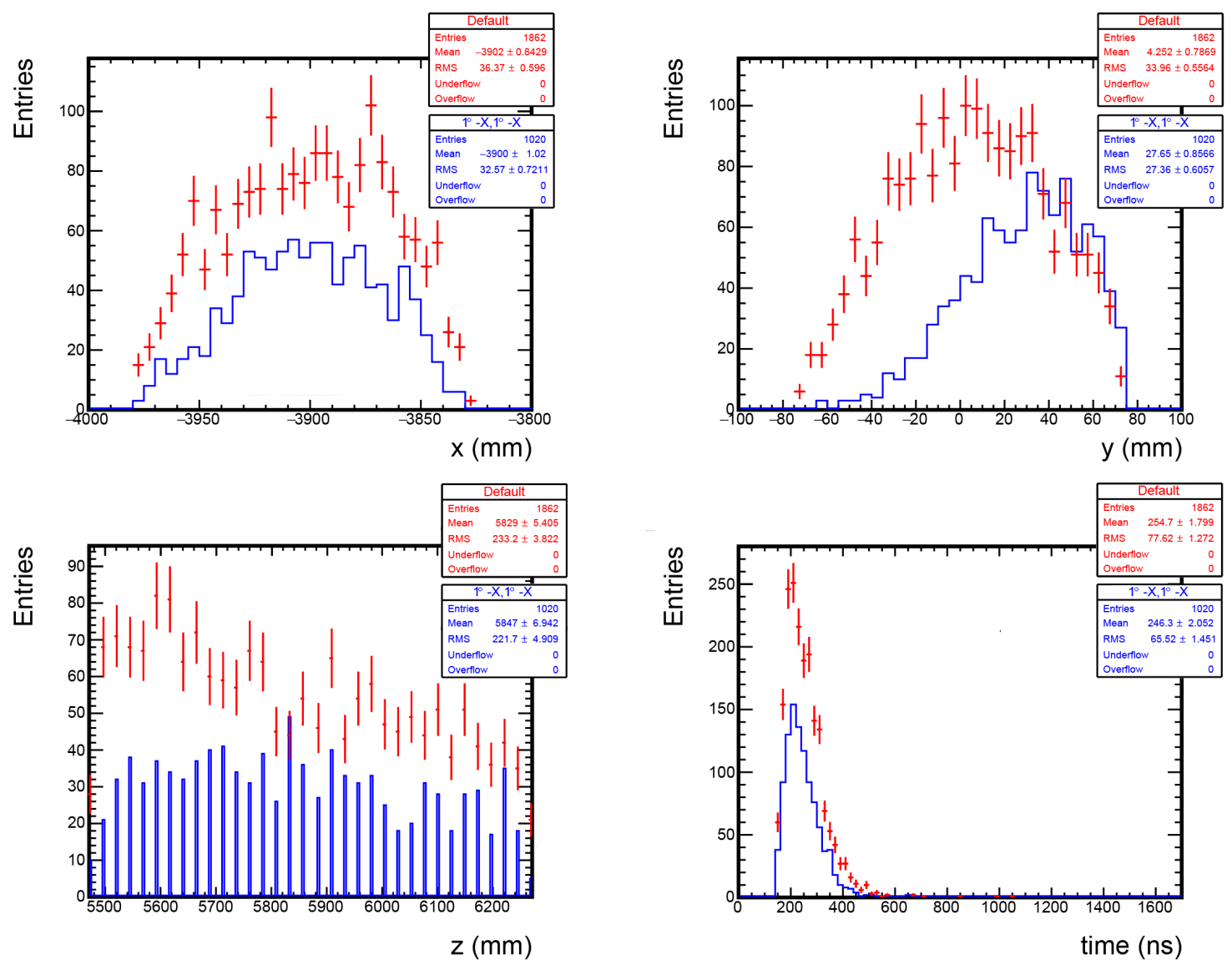

Figure 3-11: Stopped muon spatial and time distributions in Mu2e coordinate system, using SolCalc nominal field (red) and the field corresponding to a rotation of $1^{\circ}$ about $-X$ of TSu and $1^{\circ}$ about $-X$ of TSd (blue). 

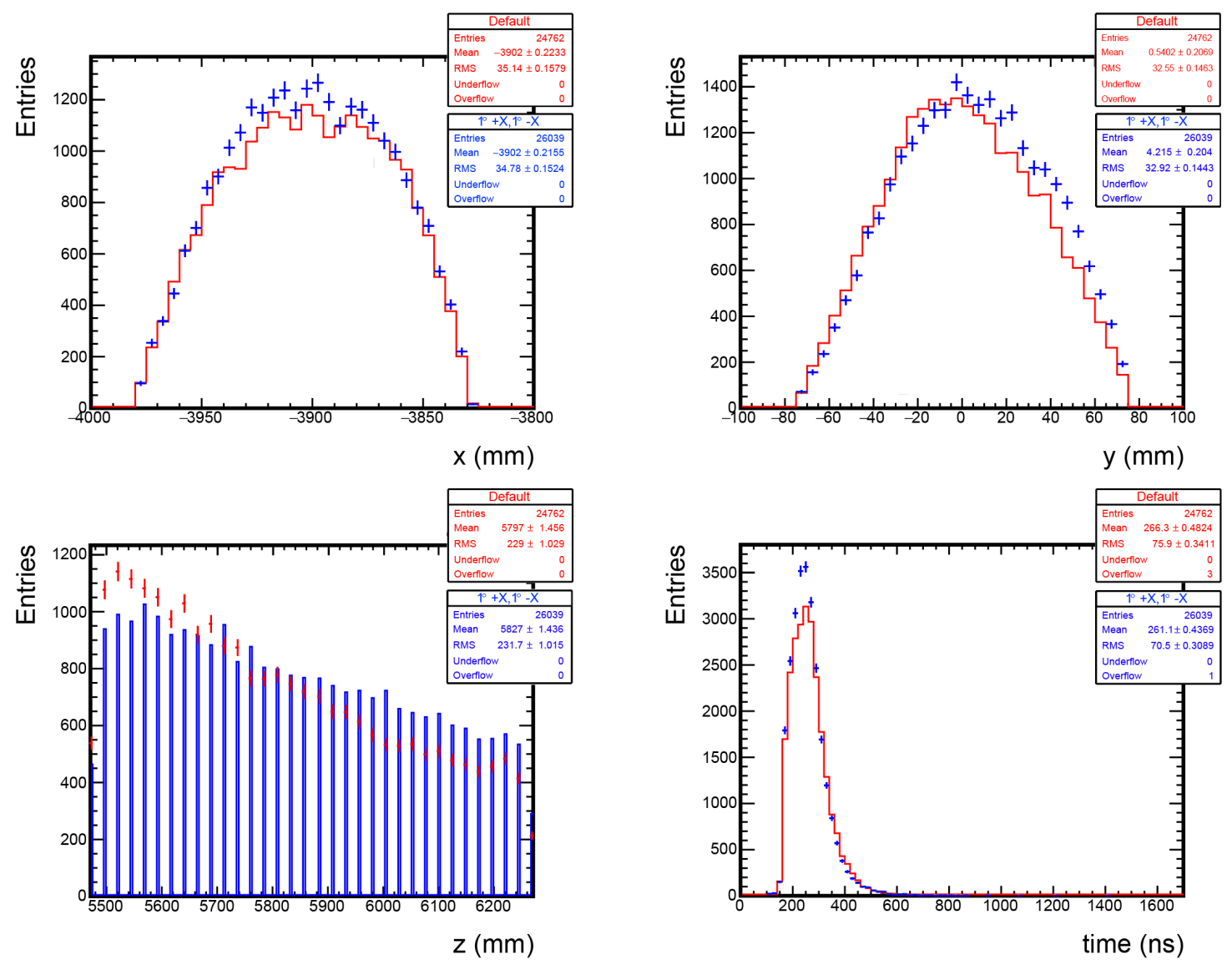

Figure 3-12: Stopped pion spatial and time distributions in Mu2e coordinate system, using SolCalc nominal field (red) and the field corresponding to a rotation of $1^{\circ}$ about $+X$ of TSu and $1^{\circ}$ about $-X$ of TSd (blue). 

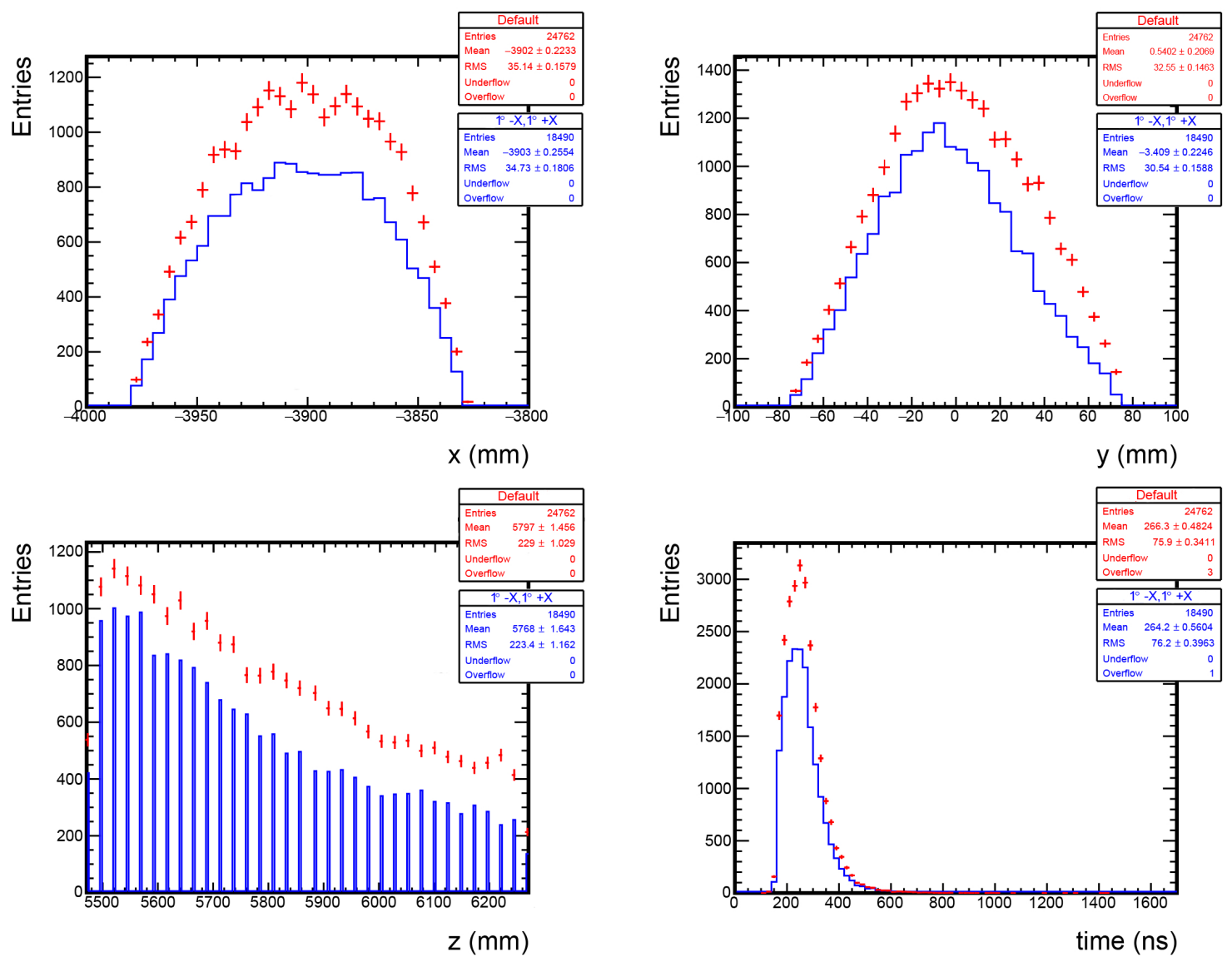

Figure 3-13: Stopped pion spatial and time distributions in Mu2e coordinate system, using SolCalc nominal field (red) and the field corresponding to a rotation of $1^{\circ}$ about $-X$ of TSu and $1^{\circ}$ about $+X$ of TSd (blue). 

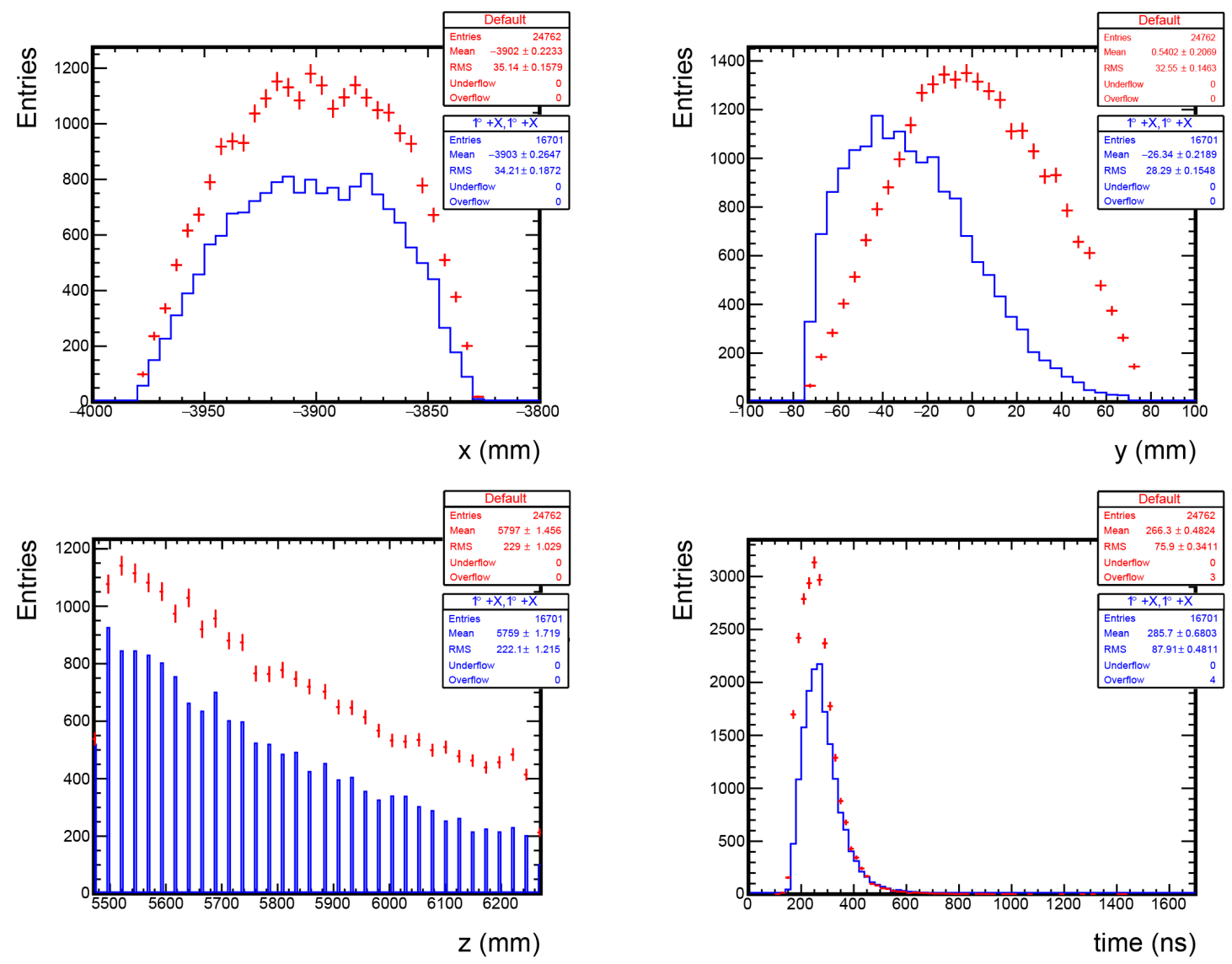

Figure 3-14: Stopped pion spatial and time distributions in Mu2e coordinate system, using SolCalc nominal field (red) and the field corresponding to a rotation of $1^{\circ}$ about $+X$ of TSu and $1^{\circ}$ about $+X$ of TSd (blue). 

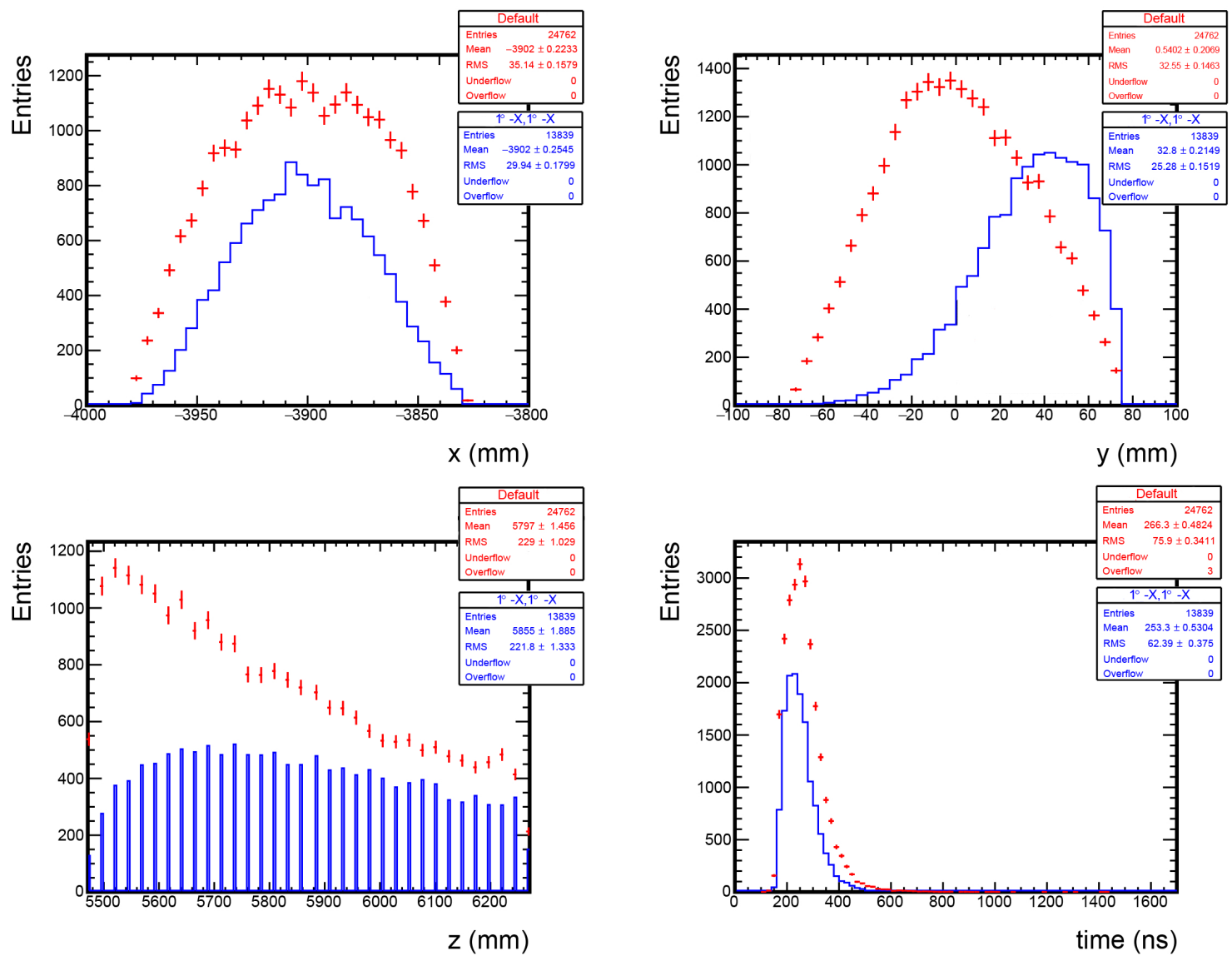

Figure 3-15: Stopped pion spatial and time distributions in Mu2e coordinate system, using SolCalc nominal field (red) and the field corresponding to a rotation of $1^{\circ}$ about $-X$ of TSu and $1^{\circ}$ about $-X$ of TSd (blue). 

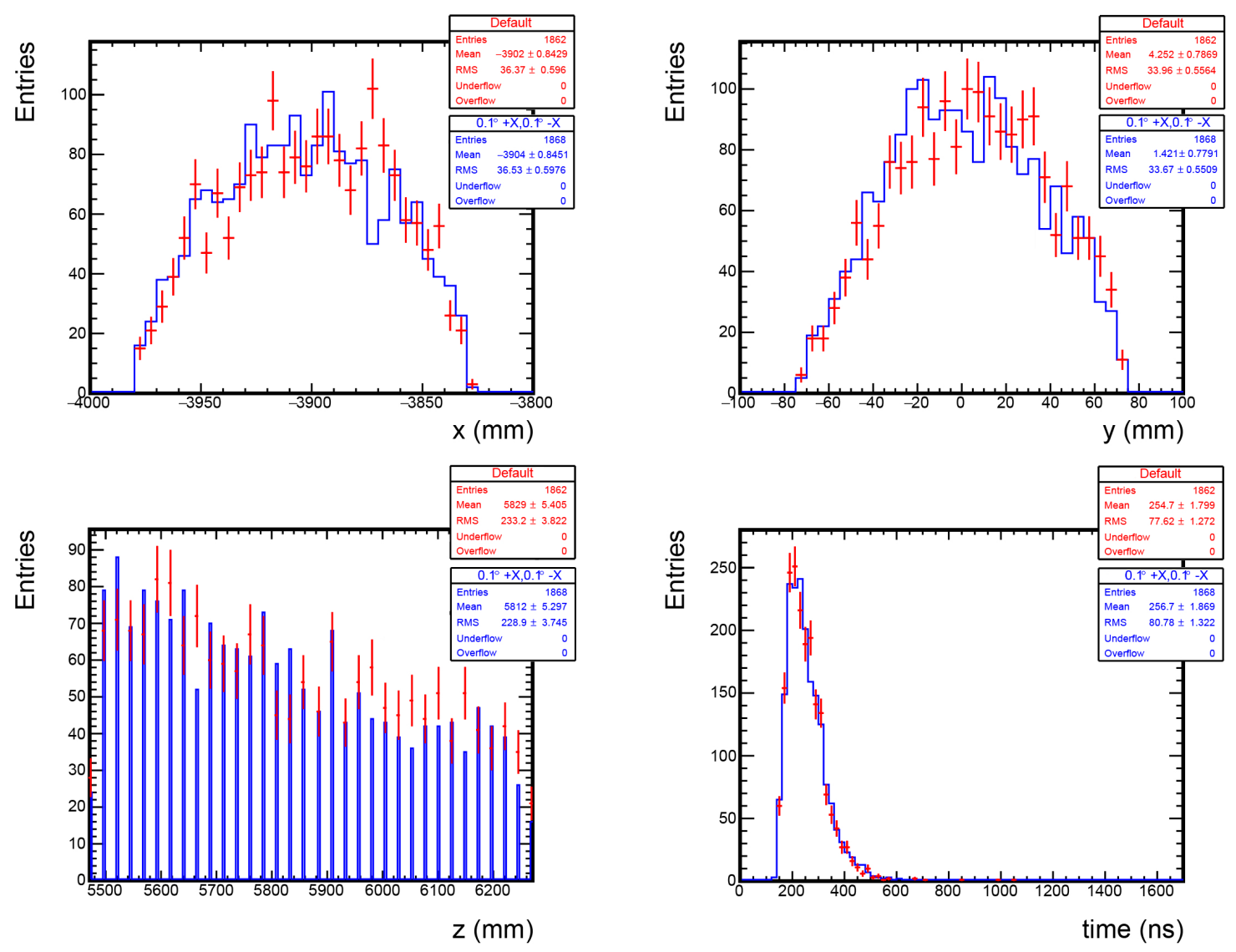

Figure 3-16: Stopped muon spatial and time distributions in Mu2e coordinate system, using SolCalc nominal field (red) and the field corresponding to a rotation of $0.1^{\circ}$ about $+X$ of TSu and $0.1^{\circ}$ about $-X$ of TSd (blue). 

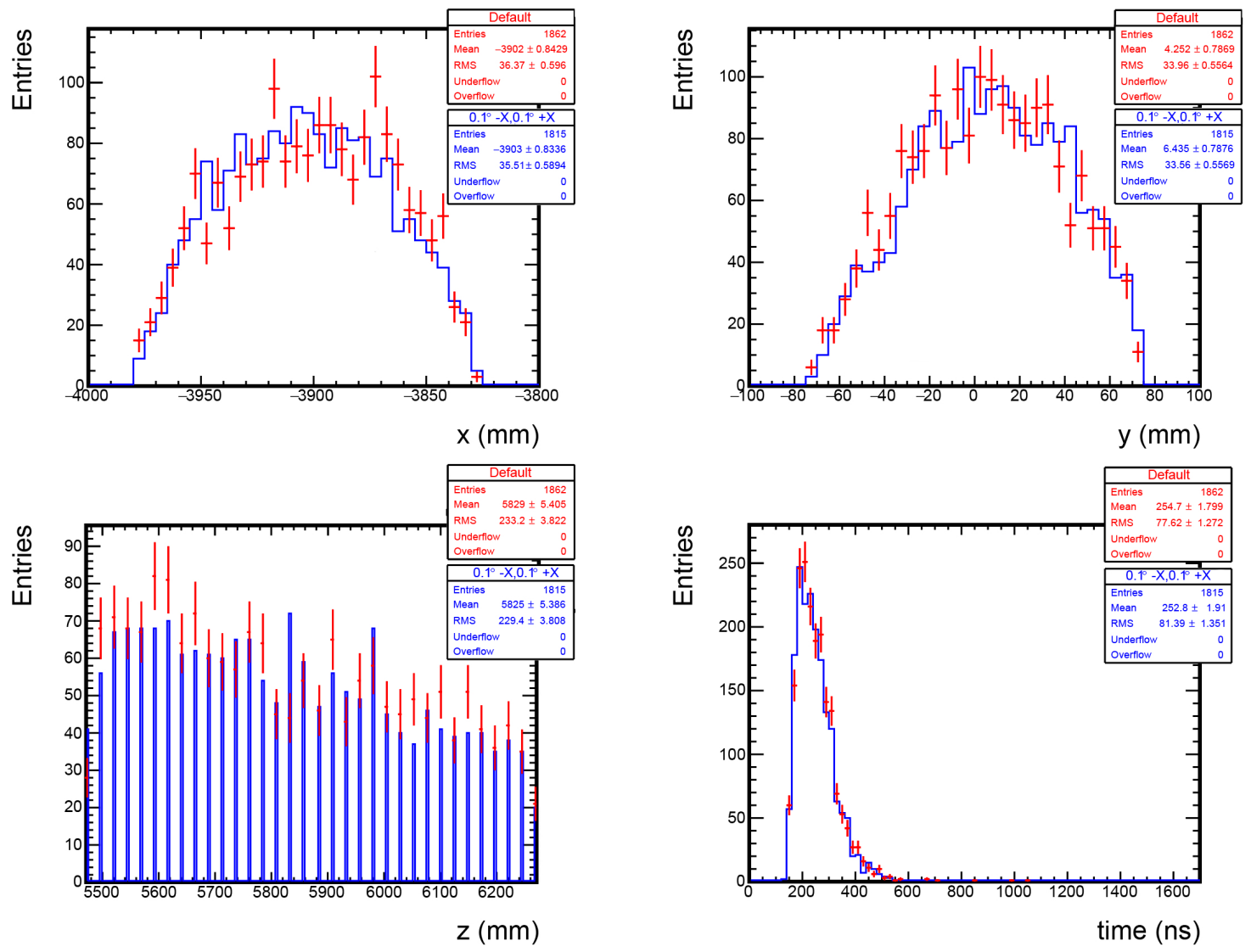

Figure 3-17: Stopped muon spatial and time distributions in Mu2e coordinate system, using SolCalc nominal field (red) and the field corresponding to a rotation of $0.1^{\circ}$ about $-X$ of TSu and $0.1^{\circ}$ about $+X$ of TSd (blue). 

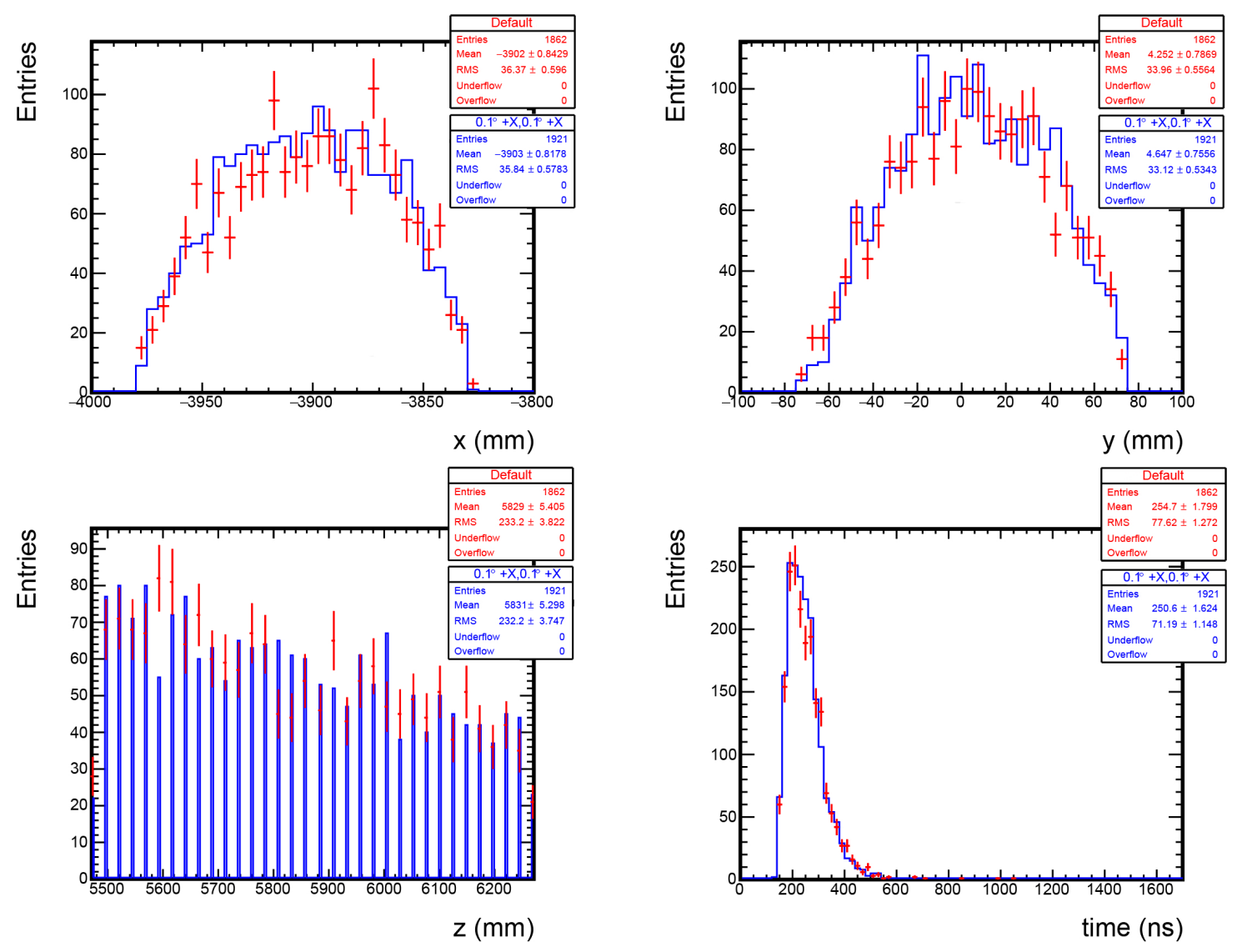

Figure 3-18: Stopped muon spatial and time distributions in Mu2e coordinate system, using SolCalc nominal field (red) and the field corresponding to a rotation of $0.1^{\circ}$ about $+X$ of TSu and $0.1^{\circ}$ about $+X$ of TSd (blue). 

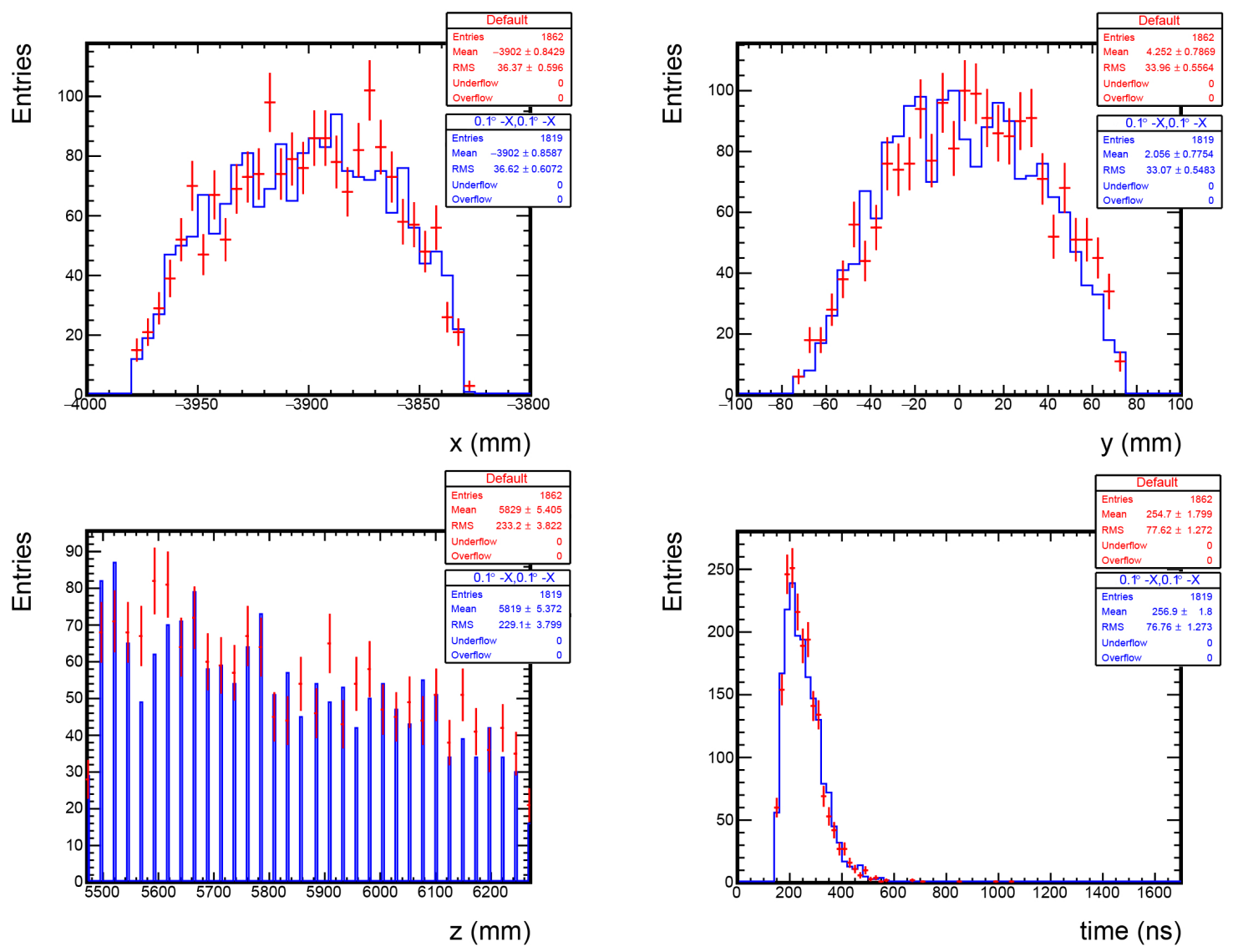

Figure 3-19: Stopped muon spatial and time distributions in Mu2e coordinate system, using SolCalc nominal field (red) and the field corresponding to a rotation of $0.1^{\circ}$ about $-X$ of TSu and $0.1^{\circ}$ about $-X$ of TSd (blue). 

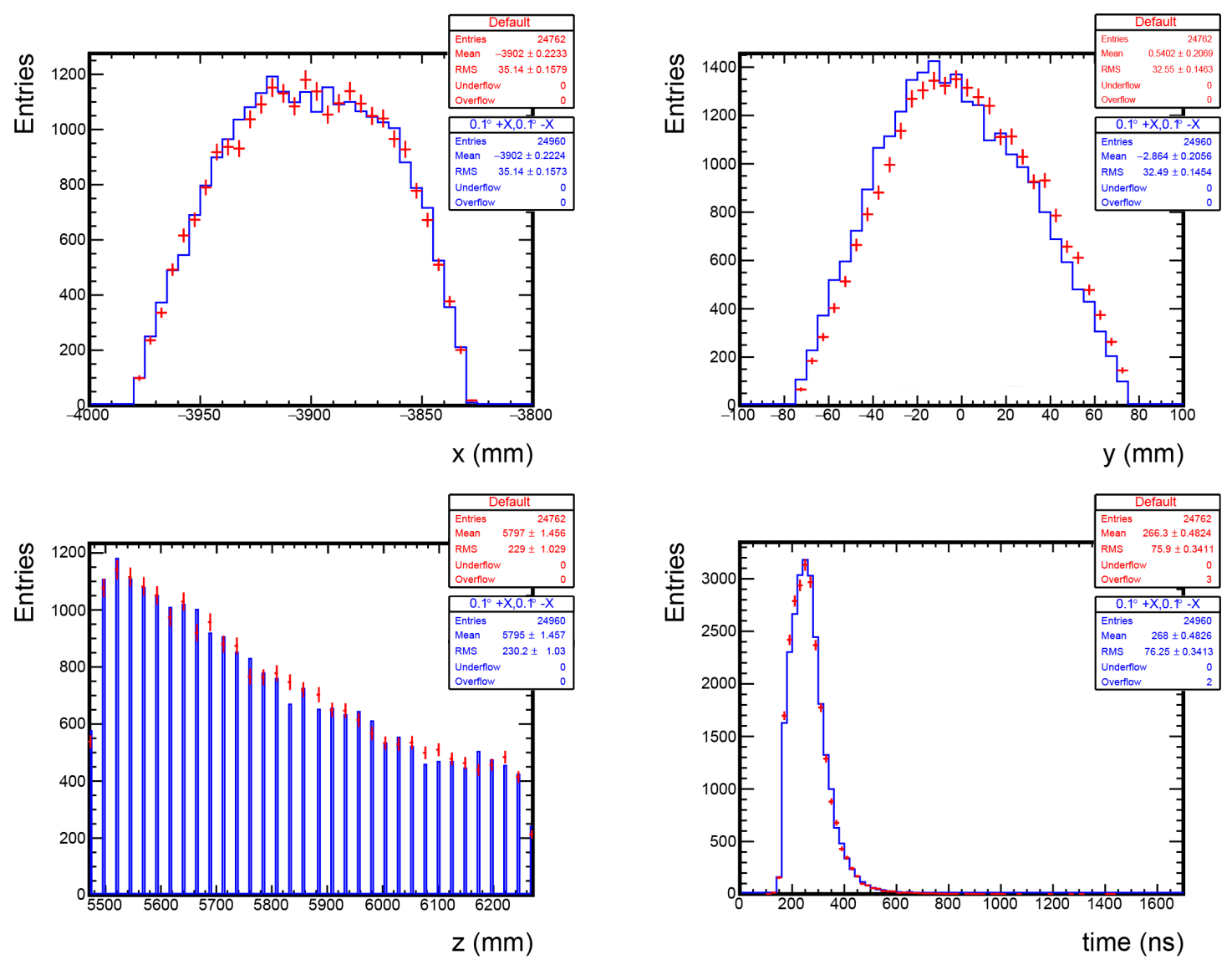

Figure 3-20: Stopped pion spatial and time distributions in Mu2e coordinate system, using SolCalc nominal field (red) and the field corresponding to a rotation of $0.1^{\circ}$ about $+X$ of TSu and $0.1^{\circ}$ about $-X$ of TSd (blue). 

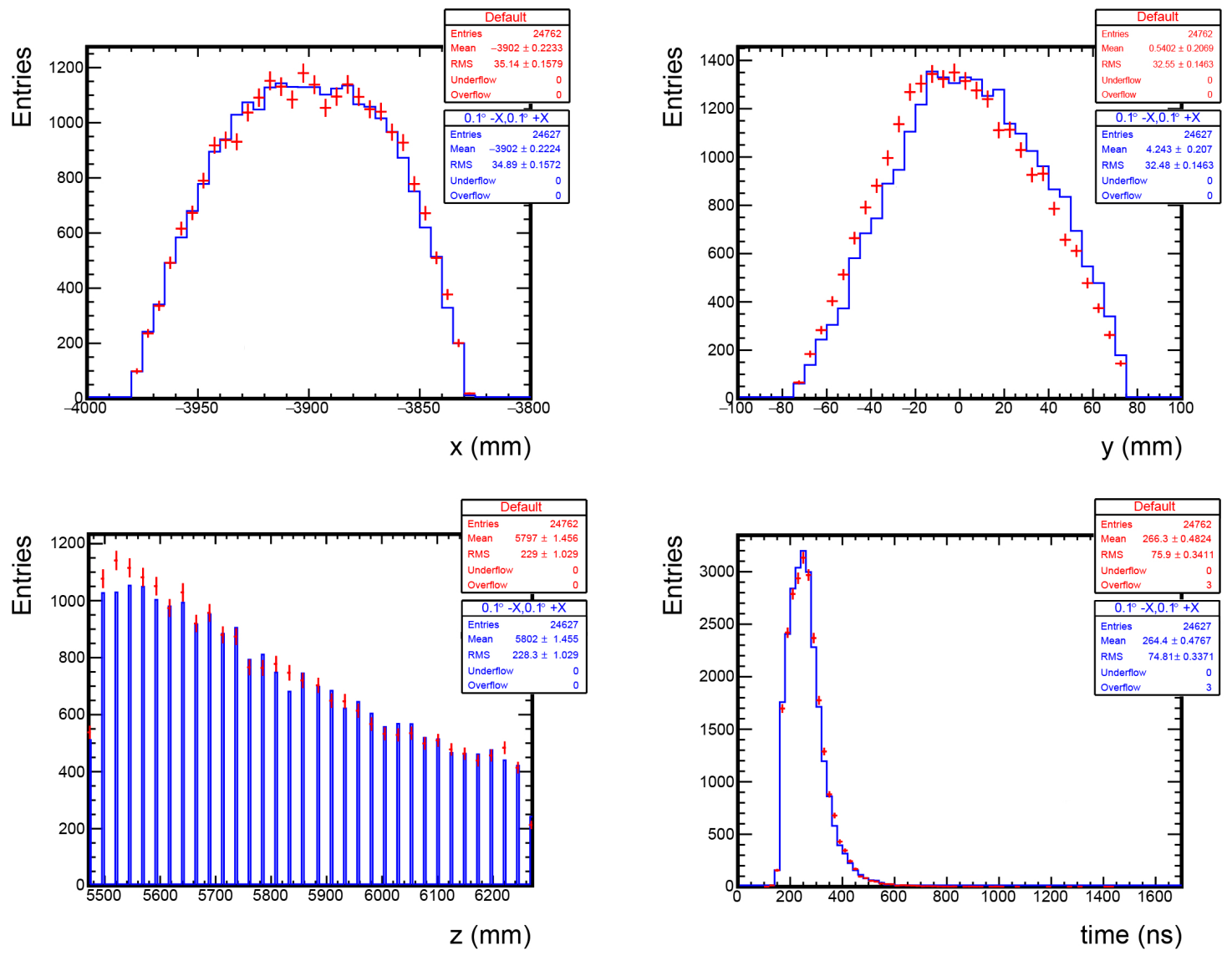

Figure 3-21: Stopped pion spatial and time distributions in Mu2e coordinate system, using SolCalc nominal field (red) and the field corresponding to a rotation of $0.1^{\circ}$ about $-X$ of TSu and $0.1^{\circ}$ about $+X$ of TSd (blue). 

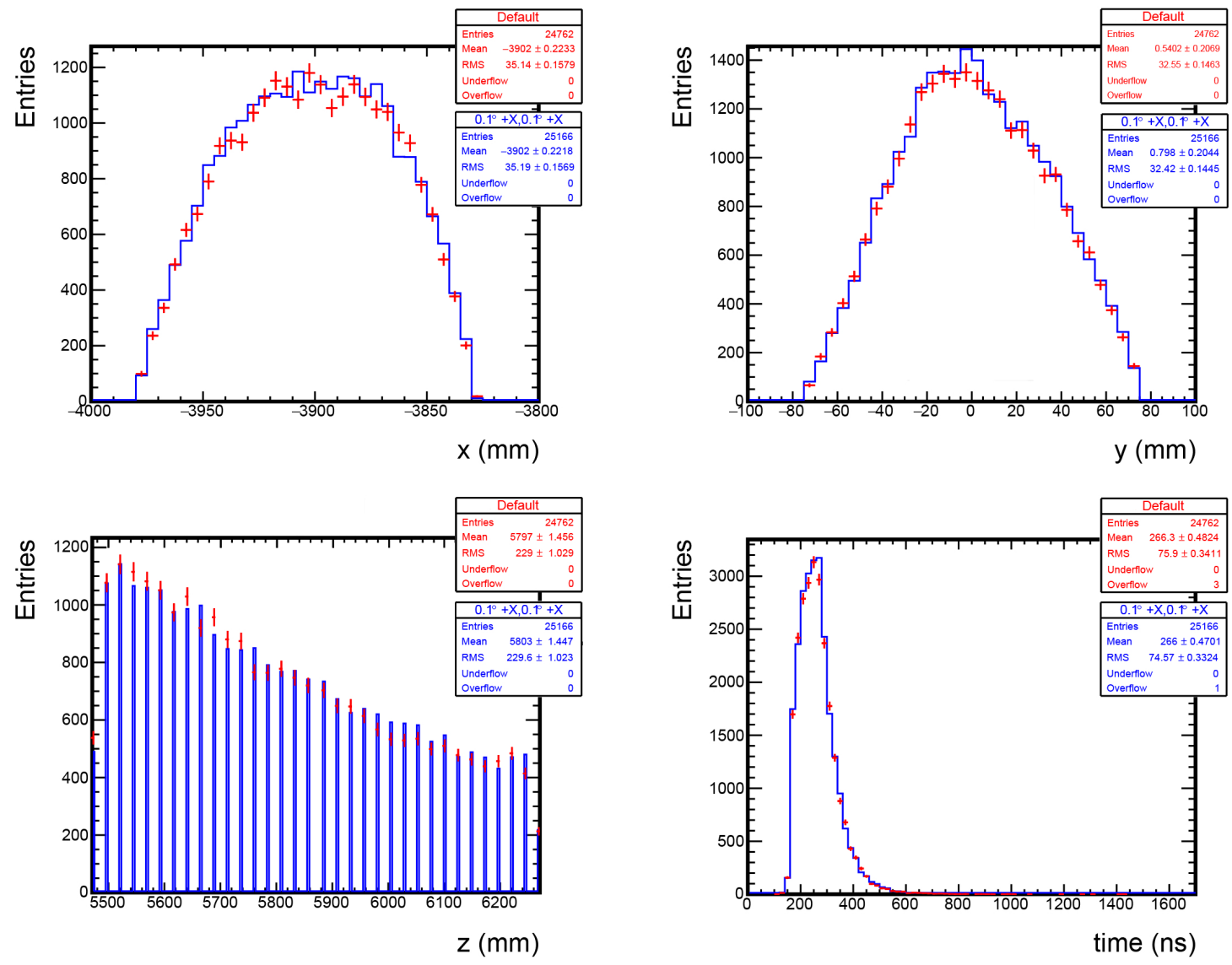

Figure 3-22: Stopped pion spatial and time distributions in Mu2e coordinate system, using SolCalc nominal field (red) and the field corresponding to a rotation of $0.1^{\circ}$ about $+X$ of TSu and $0.1^{\circ}$ about $+X$ of TSd (blue). 

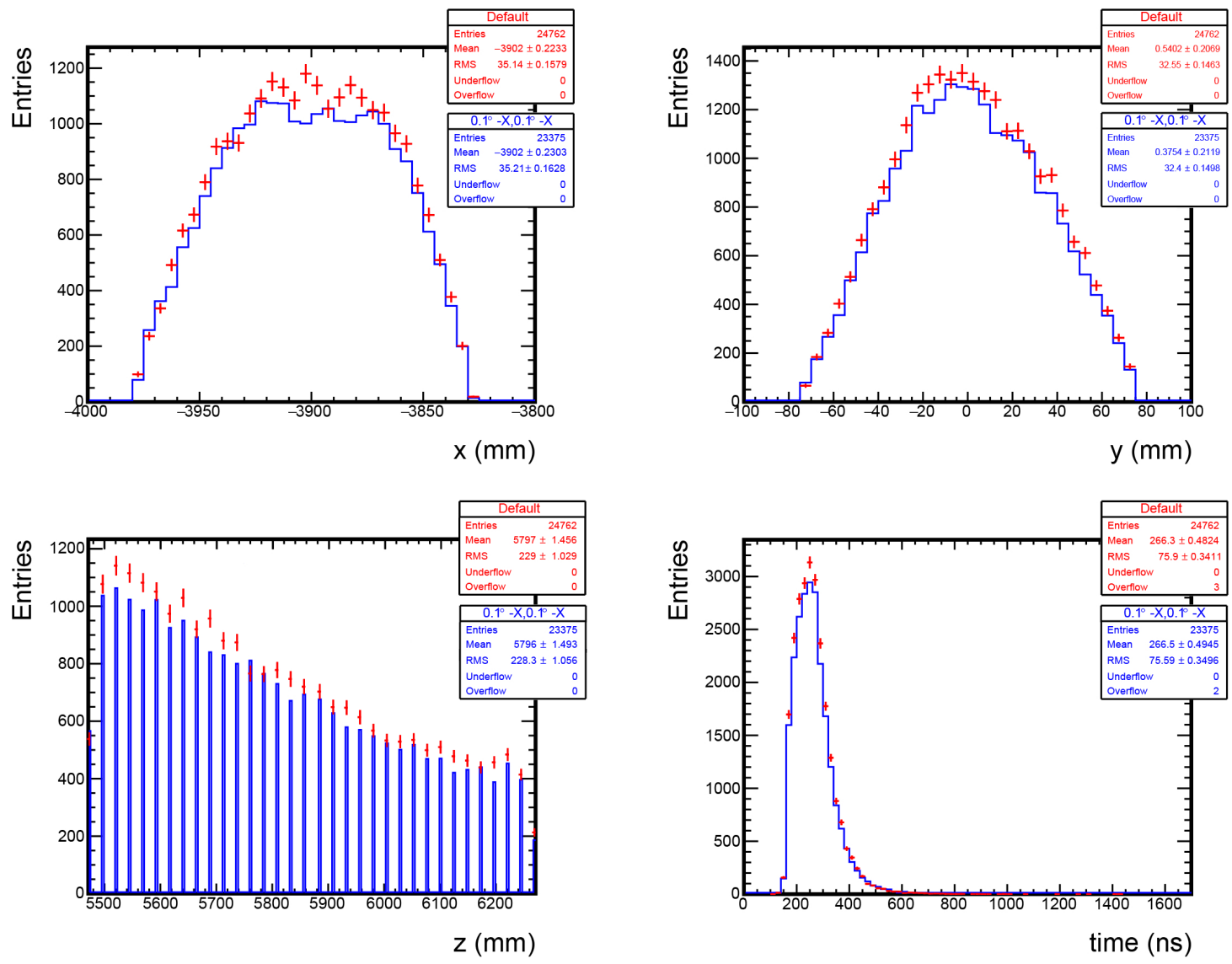

Figure 3-23: Stopped pion spatial and time distributions in Mu2e coordinate system, using SolCalc nominal field (red) and the field corresponding to a rotation of $0.1^{\circ}$ about $-X$ of TSu and $0.1^{\circ}$ about $-X$ of TSd (blue). 


\subsubsection{Parallel coil displacements}

The effects of field axis misalignments on the stopping rates is studied also for parallel coil displacements. For this study the SolCalc maps with coils shifted by $20 \mathrm{~mm}$ with respect to the $\mathrm{Y}$ axis are used in the simulation. Table 3.5 shows the muon stopping rates for the SolCalc default map and the corresponding maps with parallel coil displacements. On the average, about 2 muons are captured at the $\mathrm{Al}$ target for every 1000 protons hitting the production target. Table 3.6 shows the pion stopping rates for the same maps: the pion yields $\left(N_{\pi}\right)$ and the average arrival pion time are also shown. Up to about 100 pions are absorbed by the $\mathrm{Al}$ target for every billion of protons hitting the production target.

Table 3.5: Muon stopping rates for parallel coil displacements.

\begin{tabular}{|c|c|c|}
\hline MAPS & $N_{\mu}$ & $R_{\mu}\left(\times 10^{-3}\right)$ \\
\hline SolCalc (Mau9) DEFAult & 1862 & $1.88 \pm 0.04$ \\
\hline SolCALC (MAU9) $20 \mathrm{~mm}$ wrt +Y TSu, +20 mm wrt +Y TSd & 1919 & $1.92 \pm 0.04$ \\
\hline SolCALC (MAU9) $20 \mathrm{~mm}$ wrt -Y TSu, +20 mm wrt -Y TSd & 1903 & $1.90 \pm 0.04$ \\
\hline SolCALC (MAU9) $20 \mathrm{~mm}$ wrt +Y TSu, +20 mm wrt -Y TSd & 1939 & $1.94 \pm 0.04$ \\
\hline SolCalc (Mau9) $20 \mathrm{~mm}$ wrt -Y TSu, +20 mm wrt +Y TSd & 1892 & $1.89 \pm 0.04$ \\
\hline
\end{tabular}

Table 3.6: Pion stopping rates for parallel coil displacements.

\begin{tabular}{|c|c|c|c|}
\hline MAPS & $N_{\pi}$ & $\langle t\rangle(\mathrm{ns})$ & $R_{\pi}\left(\times 10^{-8}\right)$ \\
\hline SolCalc (MaU9) DEFAUlT & 24762 & 266.3 & $8.82 \pm 0.06$ \\
\hline SolCALC (MAU9) $20 \mathrm{~mm}$ wrt +Y TSu, +20 mm wrt +Y TSd & 25915 & 262.8 & $10.64 \pm 0.07$ \\
\hline SolCalc (MaU9) $20 \mathrm{~mm}$ wrt -Y TSu, +20 mm wrt -Y TSd & 24958 & 265.3 & $9.24 \pm 0.06$ \\
\hline SolCalc (MaU9) $20 \mathrm{~mm}$ wrt +Y TSu, +20 mm wrt -Y TSd & 24578 & 266.4 & $8.72 \pm 0.06$ \\
\hline SolCalc (MaU9) $20 \mathrm{~mm}$ wrt $-\mathrm{Y}$ TSu, $+20 \mathrm{~mm}$ wrt + Y TSd & 24863 & 265.6 & $9.10 \pm 0.06$ \\
\hline
\end{tabular}

The fractional yield differences in the rates when parallel coil displacements are induced are listed in Table 3.7. They are tiny, bigger for $\pi^{-}$than for $\mu^{-}$as in the other considered cases. Also in this case, the effect of the misalignments are evident in the $Y$ distribution, which is shifted to the right in the case of upward shifted coils. Figure 3-24 through Figure 3-27 show comparisons between $\mu^{-}$stopping rates using SolCalc default maps and SolCalc maps with parallel coil displacements; Figure 3-28 through Figure 3-31 show comparisons between $\pi^{-}$stopping rates using SolCalc default maps and SolCalc maps with parallel coil displacements. In all cases, the effects of misalignment are predominantly geometric, determined by the mismatch between the PS/TS axes and TS/DS axes 
which moves the $\mu^{-}$and $\pi^{-}$beam downwards or upwards depending on the type of varied map. The results show that in almost all cases the effect of the shifts in individual coils are statistically insignificant, even for exaggerated shifts of the order of $20 \mathrm{~mm}$. One exception is the case of all individual coils shifted above the $\mathrm{XZ}$ plane, which gives a significant increase in the total number of stopped pions. However, this variation is of the same order with the RPC background uncertainty quoted in the TDR (Table 1.1).

Table 3.7: Fractional yield differences for $\mu^{-}$and $\pi^{-}$using SolCalc maps with parallel coil displacements in TS.

\begin{tabular}{l||cc}
\hline \hline & $\mu^{-}(\%)$ & $\pi^{-}(\%)$ \\
\hline SolCalc (MaU9) $20 \mathrm{~mm}$ wrt +Y TSu, +20 mm wrt +Y TSd & $-3.06 \pm 3.35$ & $-4.67 \pm 0.93$ \\
SolCalc (MaU9) $20 \mathrm{~mm}$ wrt - Y TSu, +20 mm wrt -Y TSd & $-2.20 \pm 3.33$ & $-0.79 \pm 0.90$ \\
SolCalc (MaU9) $20 \mathrm{~mm}$ wrt +Y TSu, +20 mm wrt -Y TSd & $-4.14 \pm 3.37$ & $0.74 \pm 0.90$ \\
SolCalc (MaU9) $20 \mathrm{~mm}$ wrt - Y TSu, +20 mm wrt +Y TSd & $-1.61 \pm 3.31$ & $-0.41 \pm 0.90$ \\
\hline
\end{tabular}



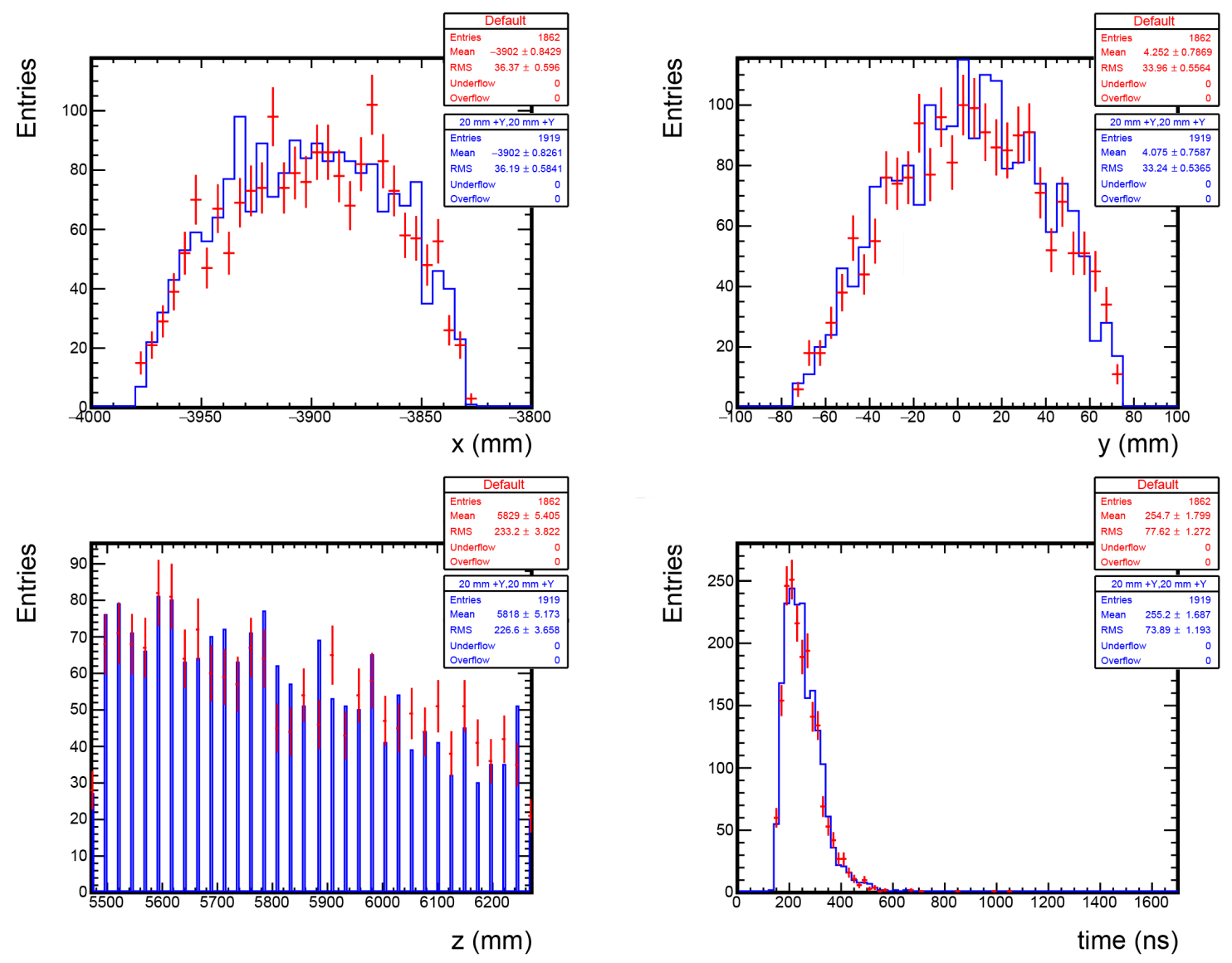

Figure 3-24: Stopped muon spatial and time distributions in Mu2e coordinate system, using SolCalc nominal field (red) and the field corresponding to parallel coil displacement of $20 \mathrm{~mm}$ about $+Y$ in TSu and about $+Y$ in TSd (blue). 

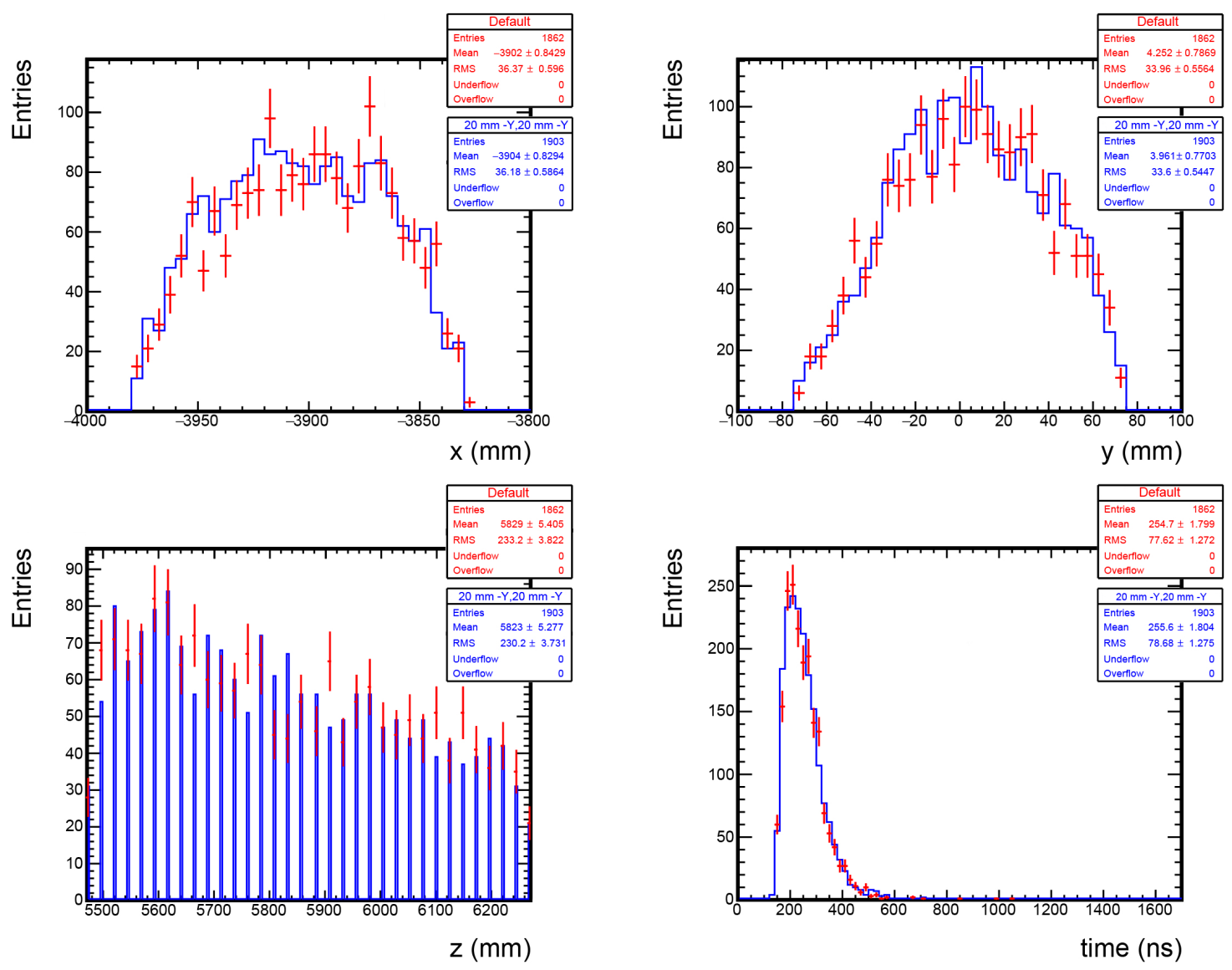

Figure 3-25: Stopped muon spatial and time distributions in Mu2e coordinate system, using SolCalc nominal field (red) and the field corresponding to parallel coil displacement of $20 \mathrm{~mm}$ about $-Y$ in TSu and about $-Y$ in TSd (blue). 

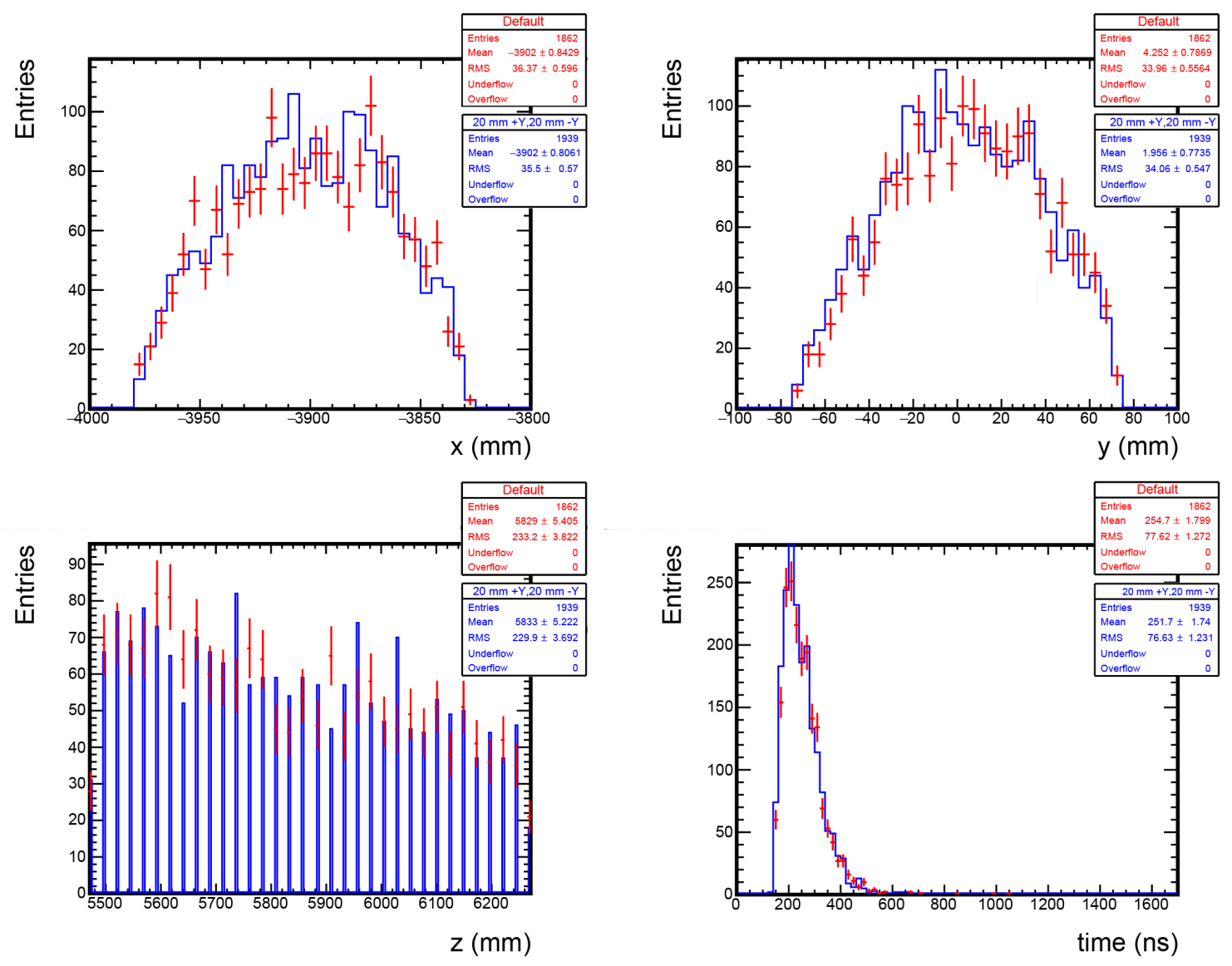

Figure 3-26: Stopped muon spatial and time distributions in Mu2e coordinate system, using SolCalc nominal field (red) and the field corresponding to parallel coil displacement of $20 \mathrm{~mm}$ about $+Y$ in TSu and about $-Y$ in TSd (blue). 

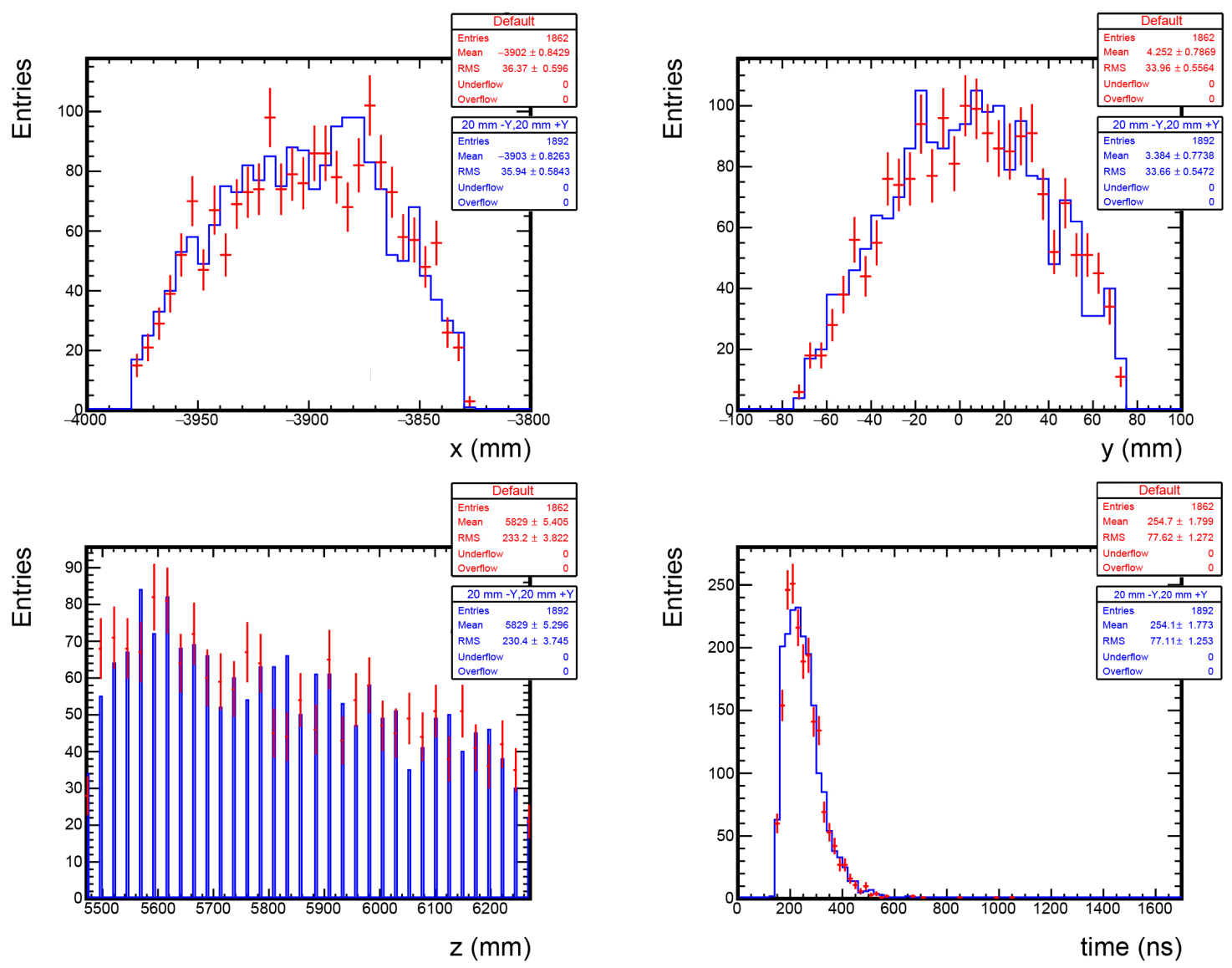

Figure 3-27: Stopped muon spatial and time distributions in Mu2e coordinate system, using SolCalc nominal field (red) and the field corresponding to parallel coil displacement of $20 \mathrm{~mm}$ about $-Y$ in TSu and about $+Y$ in TSd (blue). 

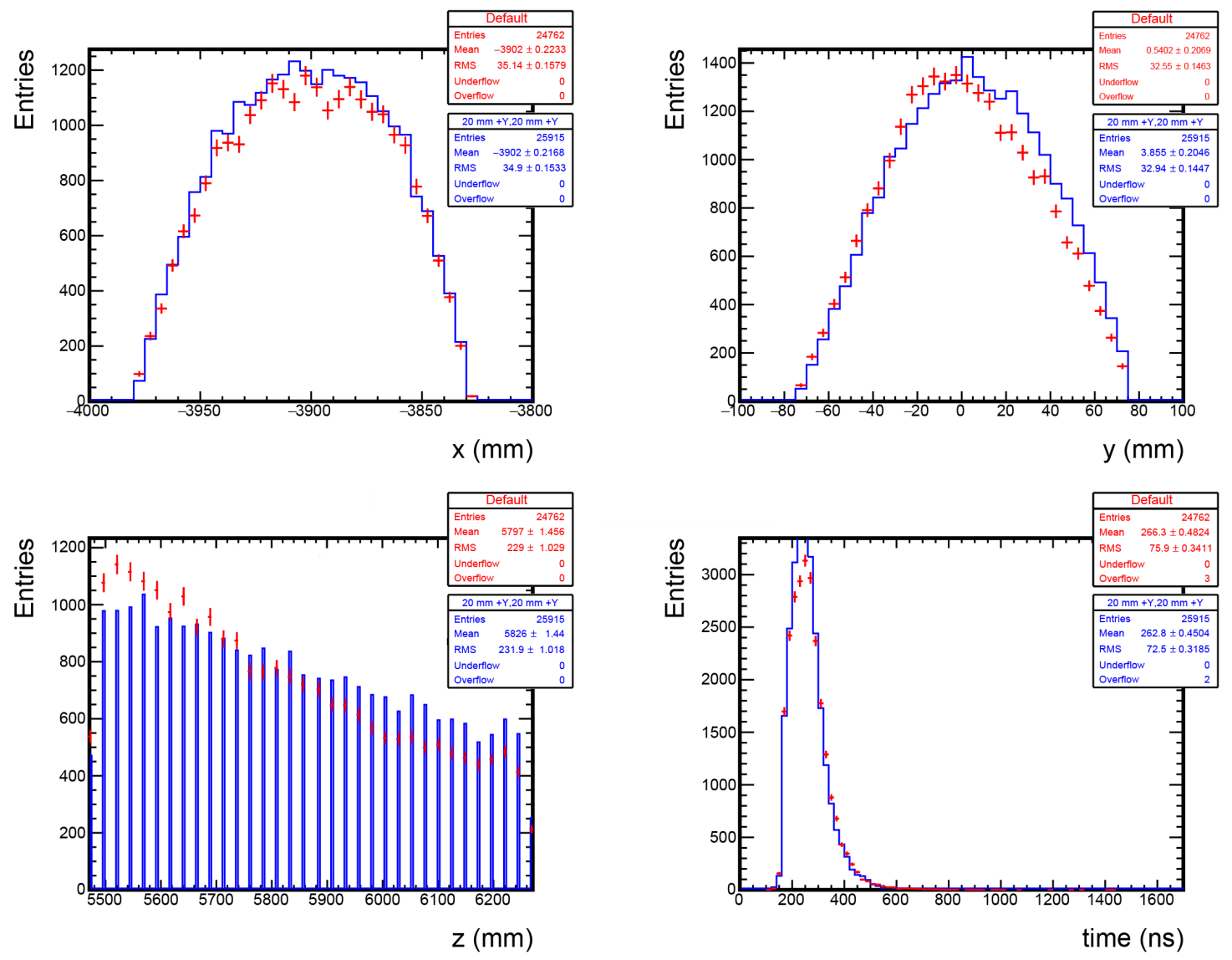

Figure 3-28: Stopped pion spatial and time distributions in Mu2e coordinate system, using SolCalc nominal field (red) and the field corresponding to parallel coil displacement of $20 \mathrm{~mm}$ about $+Y$ in TSu and about $+Y$ in TSd (blue). 

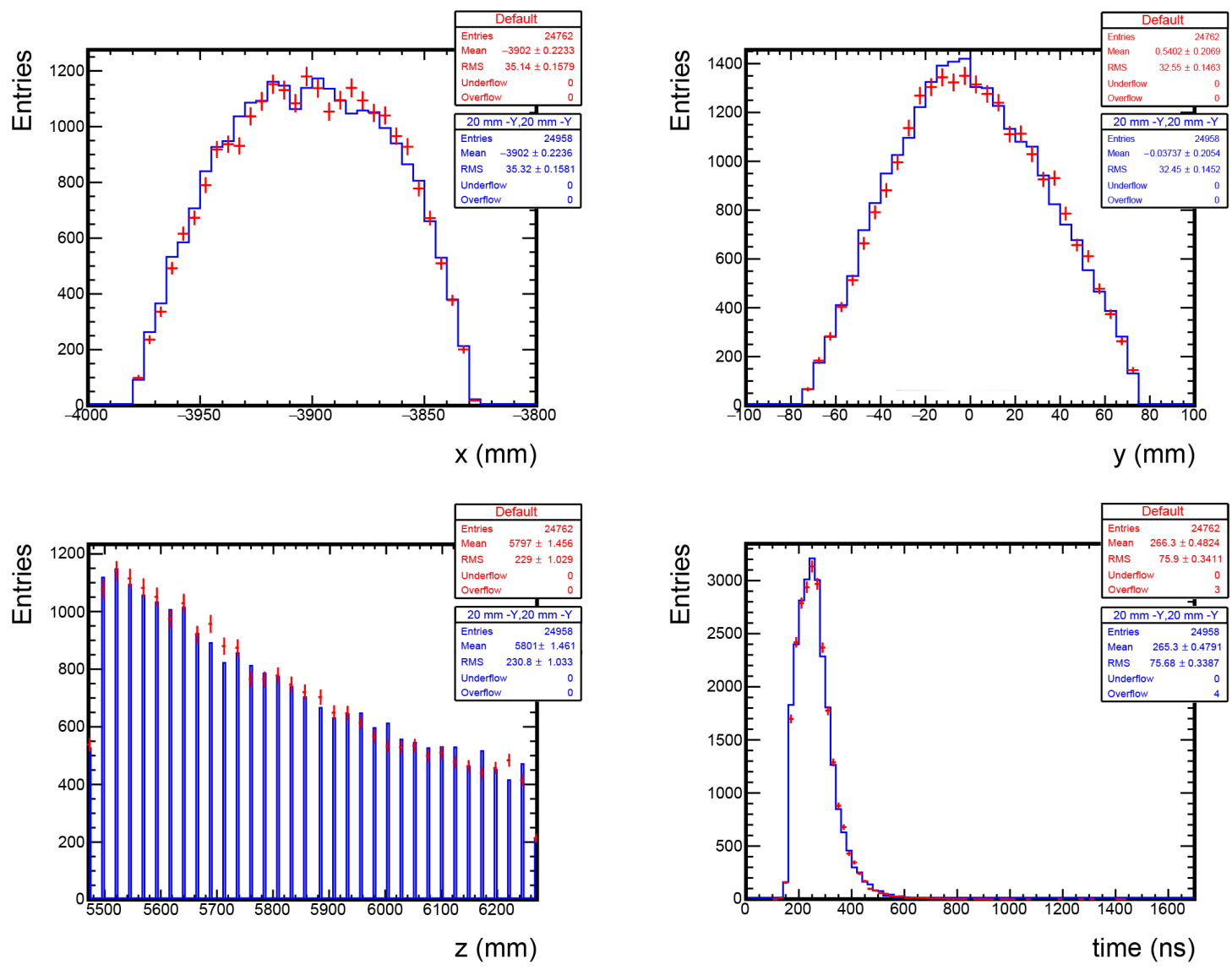

Figure 3-29: Stopped pion spatial and time distributions in Mu2e coordinate system, using SolCalc nominal field (red) and the field corresponding to parallel coil displacement of $20 \mathrm{~mm}$ about $-Y$ in TSu and about $-Y$ in TSd (blue). 

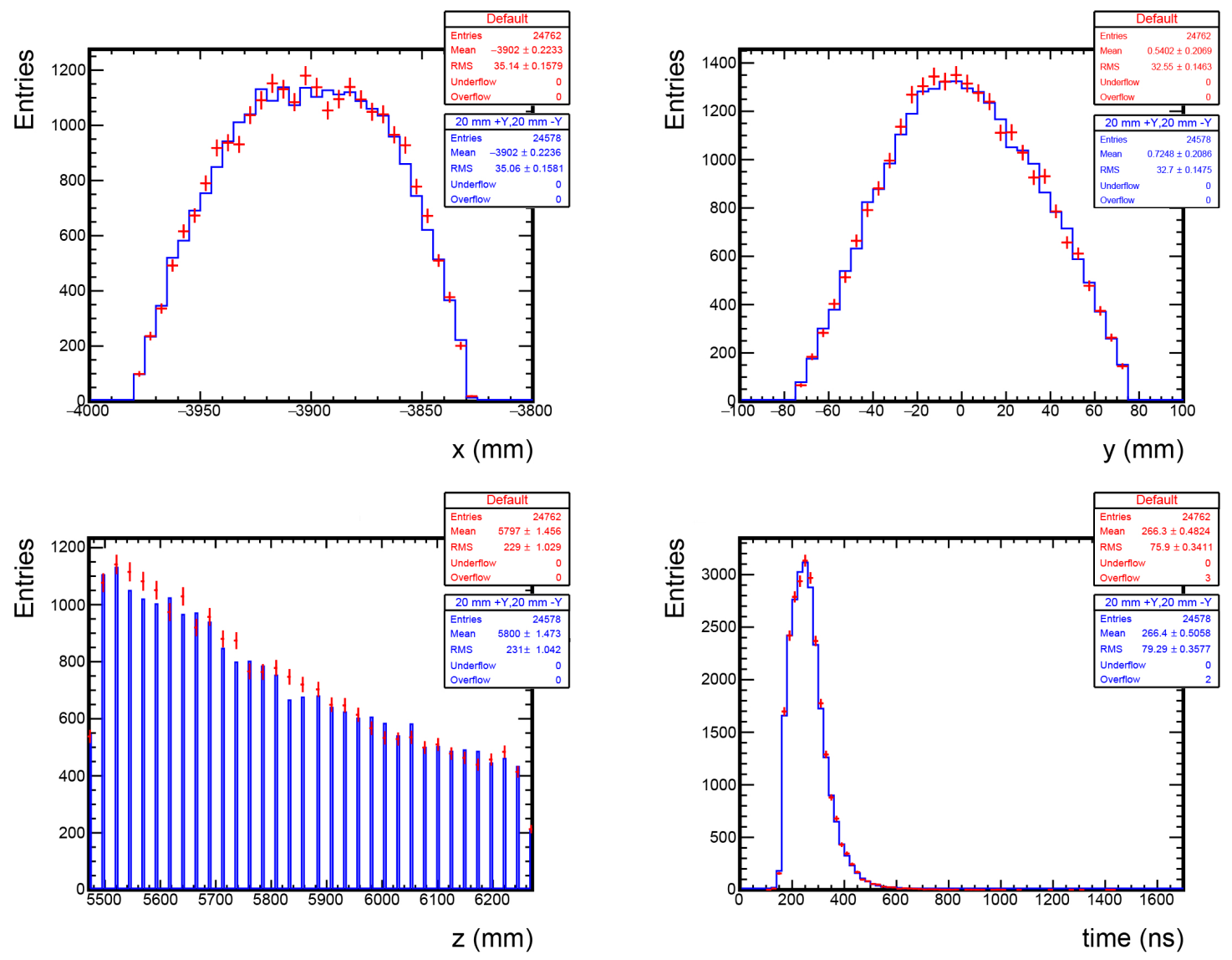

Figure 3-30: Stopped pion spatial and time distributions in Mu2e coordinate system, using SolCalc nominal field (red) and the field corresponding to parallel coil displacement of $20 \mathrm{~mm}$ about $+Y$ in TSu and about $-Y$ in TSd (blue). 

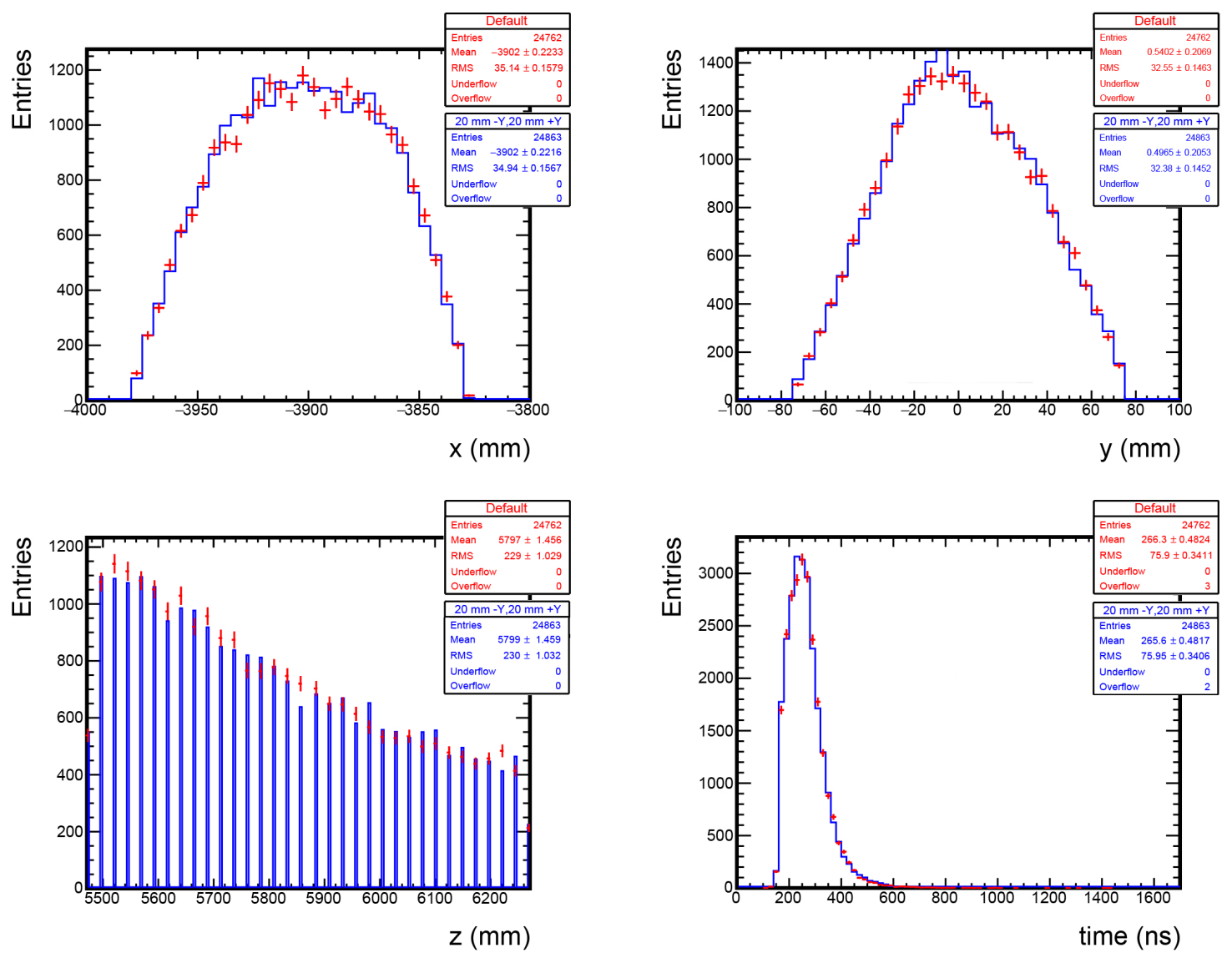

Figure 3-31: Stopped pion spatial and time distributions in Mu2e coordinate system, using SolCalc nominal field (red) and the field corresponding to parallel coil displacement of $20 \mathrm{~mm}$ about $-Y$ in TSu and about $+Y$ in TSd (blue). 


\section{CHAPTER 4}

\section{HigH MOMENTUM ELECTRON BACKGROUND}

\section{STUDIES}

One of the potential sources of background in the Mu2e experiment are beam flash electrons produced in the Production and Transport Solenoids. These electrons can be produced either from pion decays in the production target or, predominantly, from decays and scattering of particles in the Mu2e beam transport line. If high energy electrons coming from the beam enter DS and scatter to large angle off the stopping target, they can mimic the conversion electrons. Such electrons, if they appear during the measurement time window, represent direct background to $\mu \rightarrow e$ signal.

There are two ways in which this background can be reduced: through collimators and magnetic field. In the Transport Solenoid there are collimators designed to suppress the transport of particles with momenta above $100 \mathrm{MeV} / \mathrm{c}$. The magnetic field magnitude B drops in the upstream section of DS. According to Equation 1.6, this drop accelerates the electron drift downstream to the detector area, keeping the total momentum $p$ fixed and thus increasing the pitch $p_{z} / p$. In this way, the electron tracks are squeezed into the hollow part of the detector, thus falling outside the tracker acceptance, provided that the electrons do not scatter in the stopping target. Moreover, candidate conversion electron events have to satisfy requirements using the reconstructed information from the tracker, calorimeter and CRV. To reduce beam electrons background entering DS from TS, the measured track pitch is required to lie in the range $45^{\circ}<\theta<60^{\circ}\left(0.7>p_{z} / p>0.5\right)$, where $\theta$ is the angle between $p$ and $p_{z}$. This track selection requirement is $92 \%$ efficient for the signal-like electrons. 


\subsection{High MOMENTUM ELECTRON SIMULATIONS}

The beam electron background yield was estimated with an old design for the TDR ([Bartoszek et al.(2014)]) to be $(2.6 \pm 1.4) \times 10^{-3}$, where the uncertainty includes statistical $(25 \%)$ and systematic $(50 \%)$ contributions added in quadrature. A more recent estimate with a newer design of the transport line and capture target gave a yield of $<5 \times 10^{-5}$. The goal of the following study is to repeat the last estimate and to study the effects of TS misalignments on it.
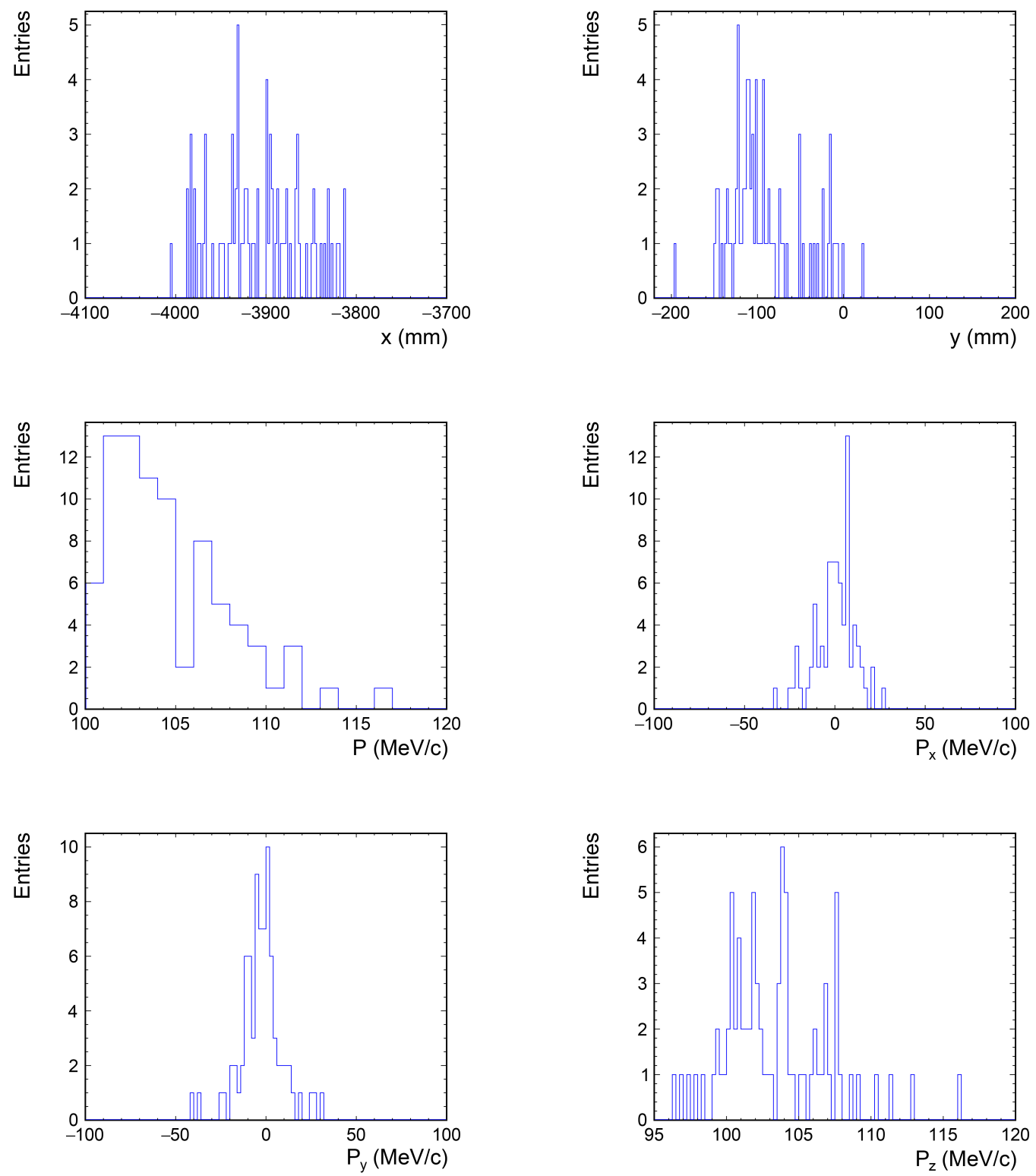

Figure 4-1: Spatial and momentum distribution of electrons after single stage simulation in the virtual detector number 9 (VD9). Mau10 default maps are used. 


\subsubsection{Tracing particles with virtual detectors}

To estimate the background from beam electrons, a single-stage simulation has been performed, using dedicated Mu2e simulation tools (see Chapter 3). It begins with $8 \mathrm{GeV}$ protons interacting in the production target and ends at the stopping target in DS. The simulation traces only electrons of energy above $100 \mathrm{MeV}$. Right at the entrance of the stopping target a virtual detector is activated: it is an ideal infinite detector, which spans all the transverse plane. The location, arrival time and momentum of electrons hitting the virtual detector are saved in a ROOT ntuple. Figure 4-1 shows the spatial and momentum distributions of electrons in the entrance of the stopping target.

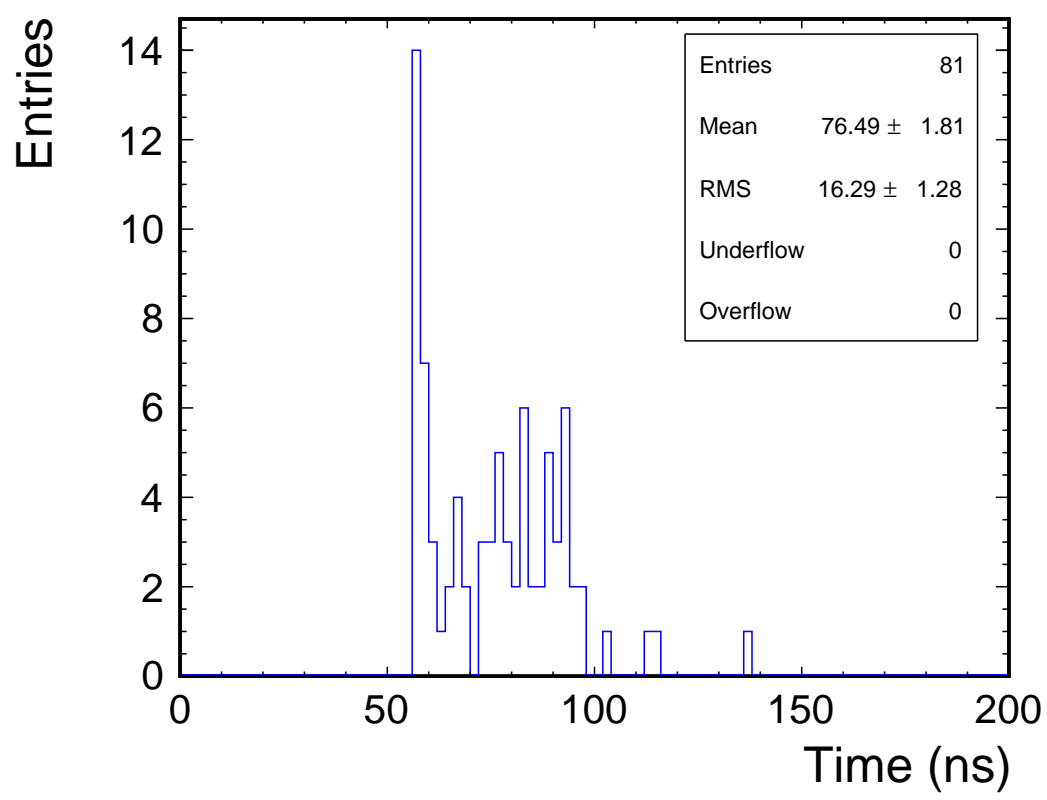

Figure 4-2: Time distribution of electrons coming from single stage simulation in VD9 using Mau10 default maps.

The plot of time distribution (Figure 4-2) shows the time arrival of high momentum electrons at the stopping target. The statistics of the simulation $\left(2.1 \times 10^{9}\right.$ POT $)$ represents an incredibly small fraction of the real experiment $\left(10^{20}\right.$ POT) and, therefore, Figure 4-2 cannot be taken as a face-value time extinction of this background. However, this figure indicates an exponential fall-off in time of the beam electron arrival at the stopping target, peaking around $50 \mathrm{~ns}$ but extending out to unlimited times (depending on electron creation and transport time through the Mu2e beam line). So an amount of this background is expected in the live time window, which has to be taken properly into account in your final estimation algorithm. For $2.1 \times 10^{9}$ simulated protons on target (POT), there are only 81 high energy electrons $(p>100 \mathrm{MeV} / \mathrm{c})$ entering the stopping target.

The angular distribution of beam electrons is concentrated at small angles with respect to the detector axis, as illustrated in Figure 4-3. Consequently, the electrons must undergo a large-angle 
scatter in materials upstream of the tracker in order to satisfy the pitch angle criteria listed above.

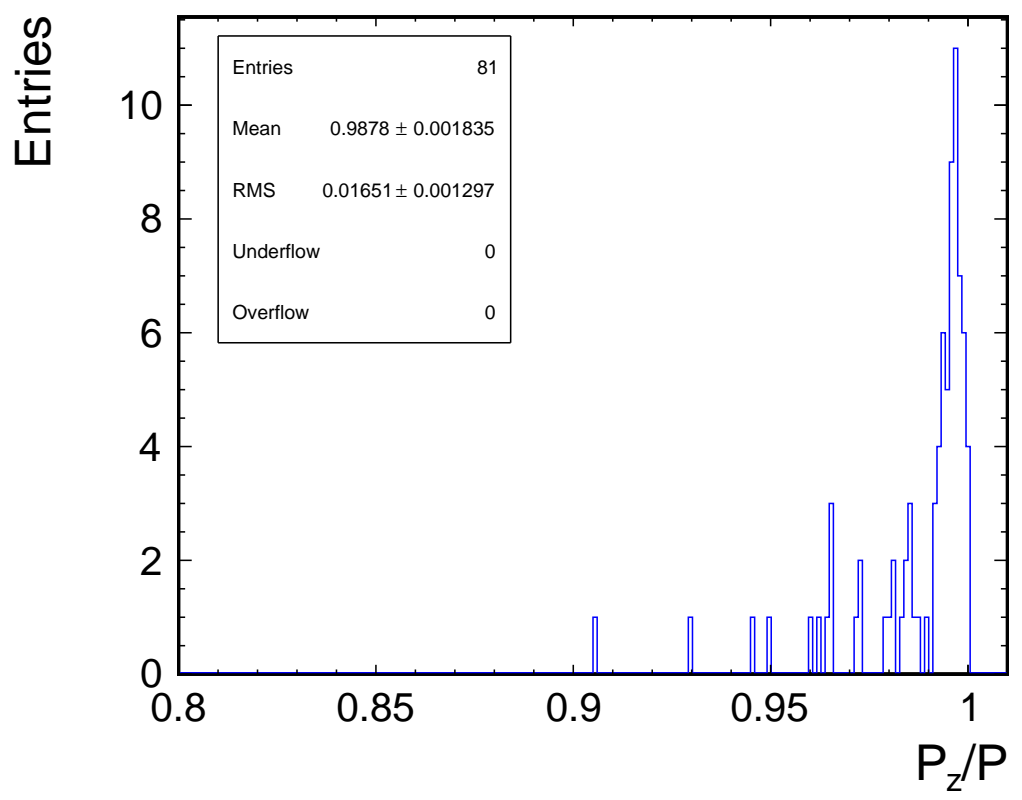

Figure 4-3: Distribution of $\cos (\theta)$ for high-momentum electrons $(p>100 \mathrm{MeV} / \mathrm{c})$ entering for DS. The pitch selection criteria require $45^{\circ}<\theta<60^{\circ}$, corresponding to $0.500<\cos (\theta)<0.707$. Mau10 default maps are used.

\subsubsection{Resampling}

In order to estimate the beam electron background, it is necessary to calculate how many of the selected high energy electrons reach the tracker. Since the sample of electrons with $p>100 \mathrm{MeV} / \mathrm{c}$ consists of only 81 events, the statistics is orders of magnitude smaller the statistics required to produce a single background event. Consequently, in order to increase the statistics, an artificial sample of high energy electrons entering DS is generated. Each high momentum electron in the original sample is replaced by a cloud of $10^{6}$ electrons whose momentum is randomized according to a probability distribution function determined from fit to the original sample. The original sample can be reused multiple times, because primary electrons do not make hits directly in the tracker, since they have to undergo rare large-angle scattering. Thus the resampling approach is valid, since rare processes are looked for. Figure 4-4 shows the momentum histogram of the original 81 electrons and the fit used for the resampling, with the corresponding uncertainties taken as totally uncorrelated. The original momentum $p$ is replaced by the momentum distribution:

$$
f(p)=A \cdot e^{-k\left(p-p_{0}\right)}
$$

where $A=25.3 \pm 6.3, k=0.22 \pm 0.061 / \mathrm{MeV}$ and $p_{0}=100 \mathrm{MeV} / \mathrm{c}$. The momentum components 
$p_{x}, p_{y}, p_{z}$ are rescaled by the common factor $p_{\text {resampled }} / p_{\text {original }}$. The position coordinates and time are randomized as well according to a Gaussian distribution with $\sigma=10 \mathrm{~mm}$ and $\sigma=10 \mathrm{~ns}$ respectively.

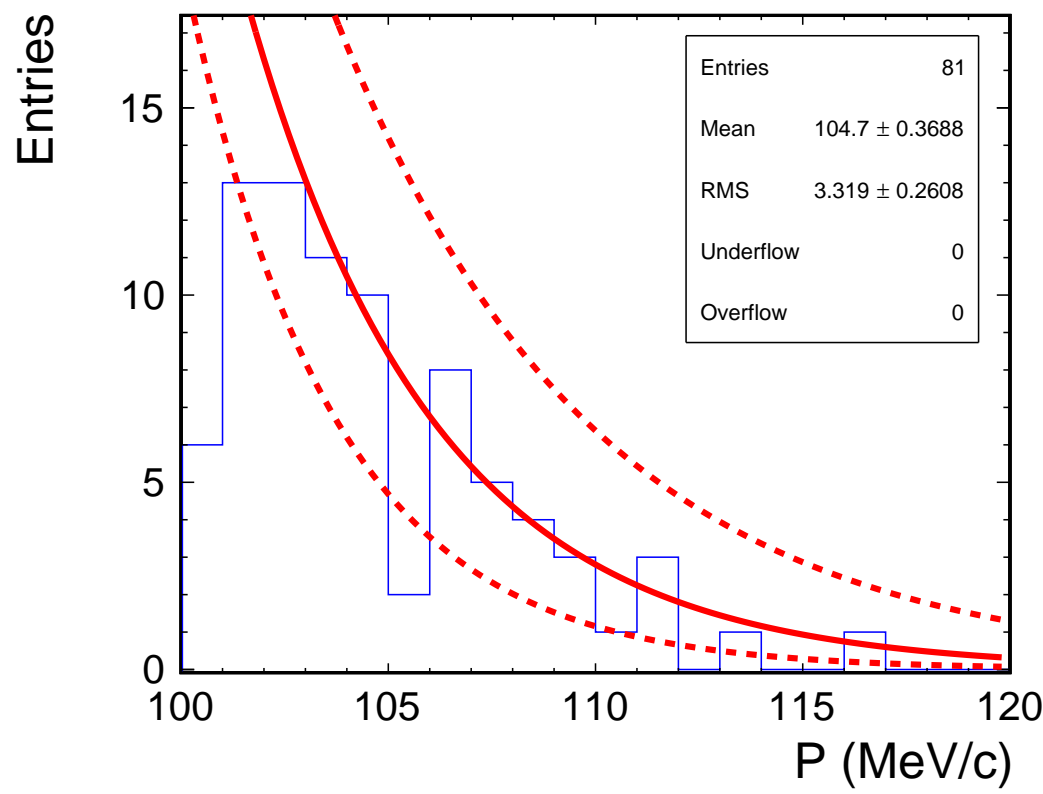

Figure 4-4: Momentum magnitude histogram of the high energy electrons coming from the single stage simulation using Mau10 default maps. The red full line shows the fit function, the dotted ones the corresponding uncertainties. The uncertainties are taken as totally uncorrelated.

Table 4.1: Results of the simulation using Mau10 default fields, after resampling, with the nominal fitting function and its variations within the fitting uncertainties.

\begin{tabular}{l||cc}
\hline \hline \multirow{2}{*}{ FIT } & \multicolumn{2}{c}{$e^{-}$in the tracker } \\
& BEFORE cuts & AFTER cuts \\
\hline Default & 4106 & 27 \\
Up & 3973 & 24 \\
Down & 3925 & 17 \\
\hline \hline
\end{tabular}

Table 4.1 shows the results obtained with the resampled electrons, before and after the cuts on the tracker hits (see subsection 4.1.3), using the nominal fitting function and its variants resulting by varying the parameters of Equation 4.1 within their fitting uncertainties, assumed totally anticorrelated (this assumption gives the maximum variation). Within statistical uncertainties, the results are consistent among all three cases, both before and after cuts. In fact, it has been observed that the choice of the fitting function does not affect significantly the results of the background estimation in all cases examined in this study. Therefore, the choice of the fitting function derived 
from the resampled electrons plays a minor role, a fact that justifies the applied methodology of resampling and fitting.

Figure 4-5 shows the coordinate and momentum distributions of electrons in VD9 after resampling. The $X$ and $Y$ distributions are smoothed, because of the gaussian smearing applied on the original sample. The momentum magnitude shape inherits the smoothness of the fitting curve. Since the $p_{z}$ distribution follows very closely the magnitude distribution ( $p$ is mostly $p_{z}$ ), it is smooth too. Only $p_{z}$ and $p_{y}$ retain the spiky shapes of their original histograms after resampling, because they are only rescaled without smearing.
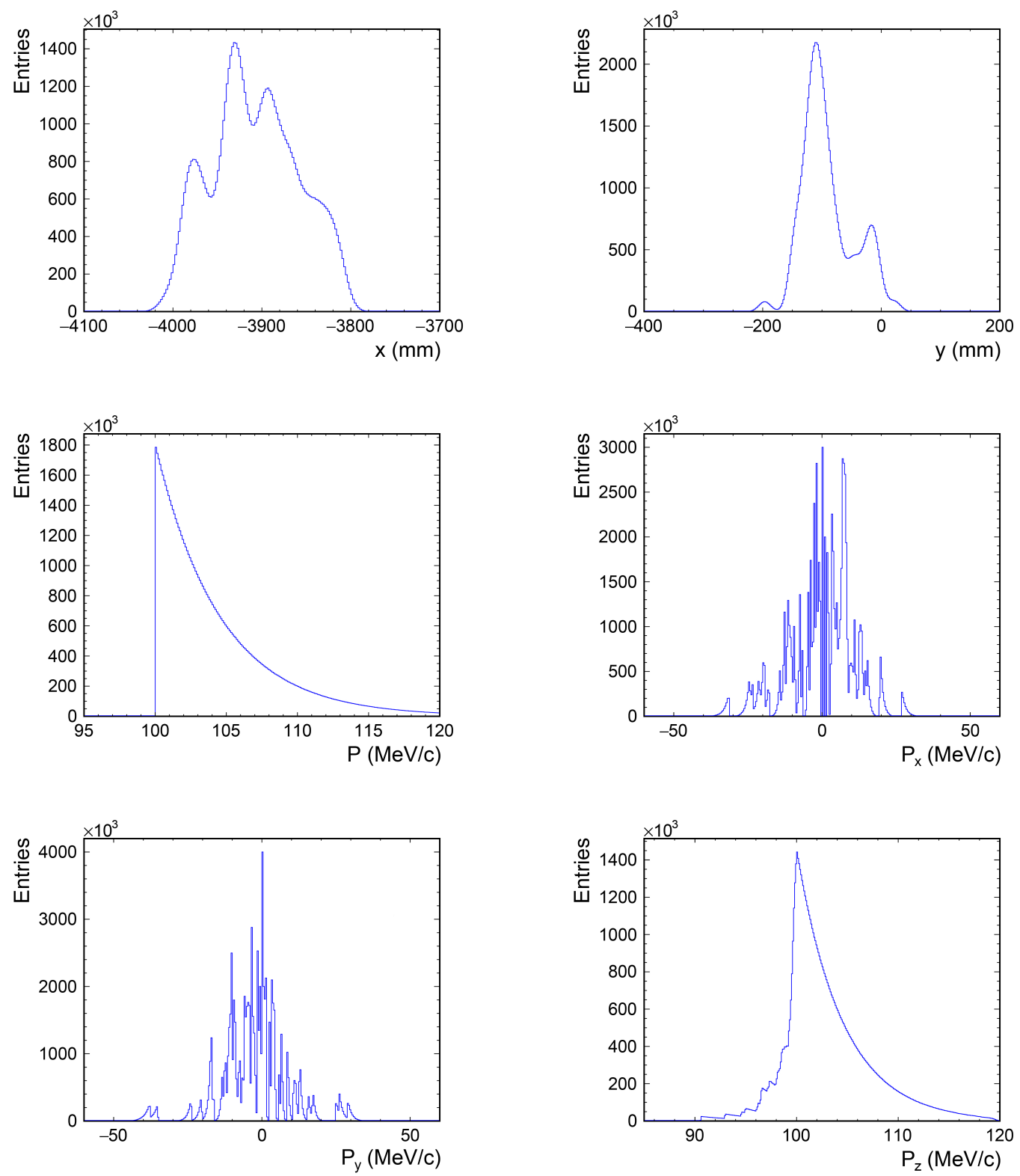

Figure 4-5: Spatial and momentum distribution of electrons in VD9 after resampling using Mau10 default maps. 


\subsubsection{Stage 4 simulation}

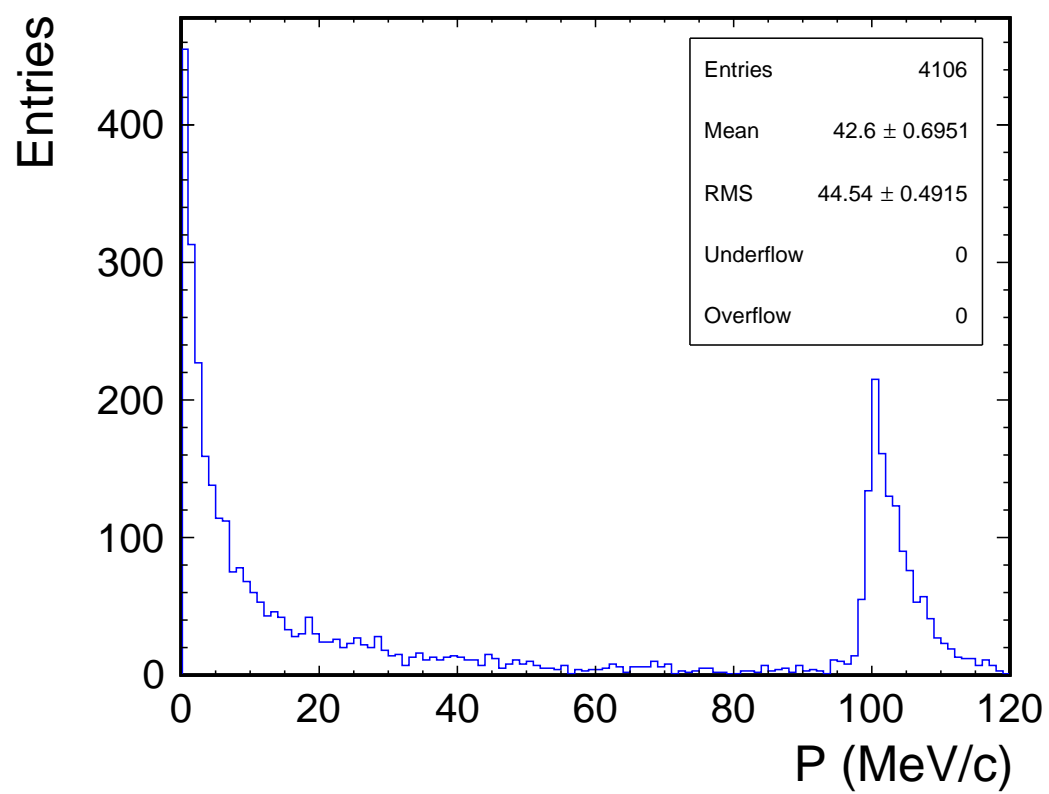

Figure 4-6: Momentum magnitude distribution of all beam flash electrons which hit the inclusive tracker volume. Mau10 default maps are used in the simulation.

After resampling, the resulting dataset is passed again through the GEANT4 simulation: electrons are sent through the stopping target and the ones which cross the inclusive tracker volume are recorded. the electron tracks are not reconstructed in the tracker: the simulation only records electron hits in the detector. Only 4106 electrons hit the tracker. The momentum distribution of all electrons hitting the inclusive tracker volume is shown in Figure 4-6: the momentum magnitude shape shows that relatively low momentum electrons coming from $\gamma$ rays (converted to $e^{+} e^{-}$) and from $\delta$ rays (energetic electrons knocked out of atoms), while the primary ones peak on the high momentum side. The location of points where the electron crosses the tracker for the first time (Figure 4-7) is very non-uniform in the $X Y$ plane: in particular, an asymmetry in $Y$ is observed, since the electrons are coming mostly from below the $\mathrm{XZ}$ plane. Some electrons pass through all the tracker, but a more detailed analysis with selection criteria is required in order to know if these are reconstructable signals. The angle distribution in Figure 4-8 shows that electrons which cross the tracker have large $p_{z} / p$; also, as shown in the scatter plot on the right, the signal-like electrons have high $p_{T}$, while all of the events at small $p_{z}$ and $p_{T}$ are secondary produced particles. 

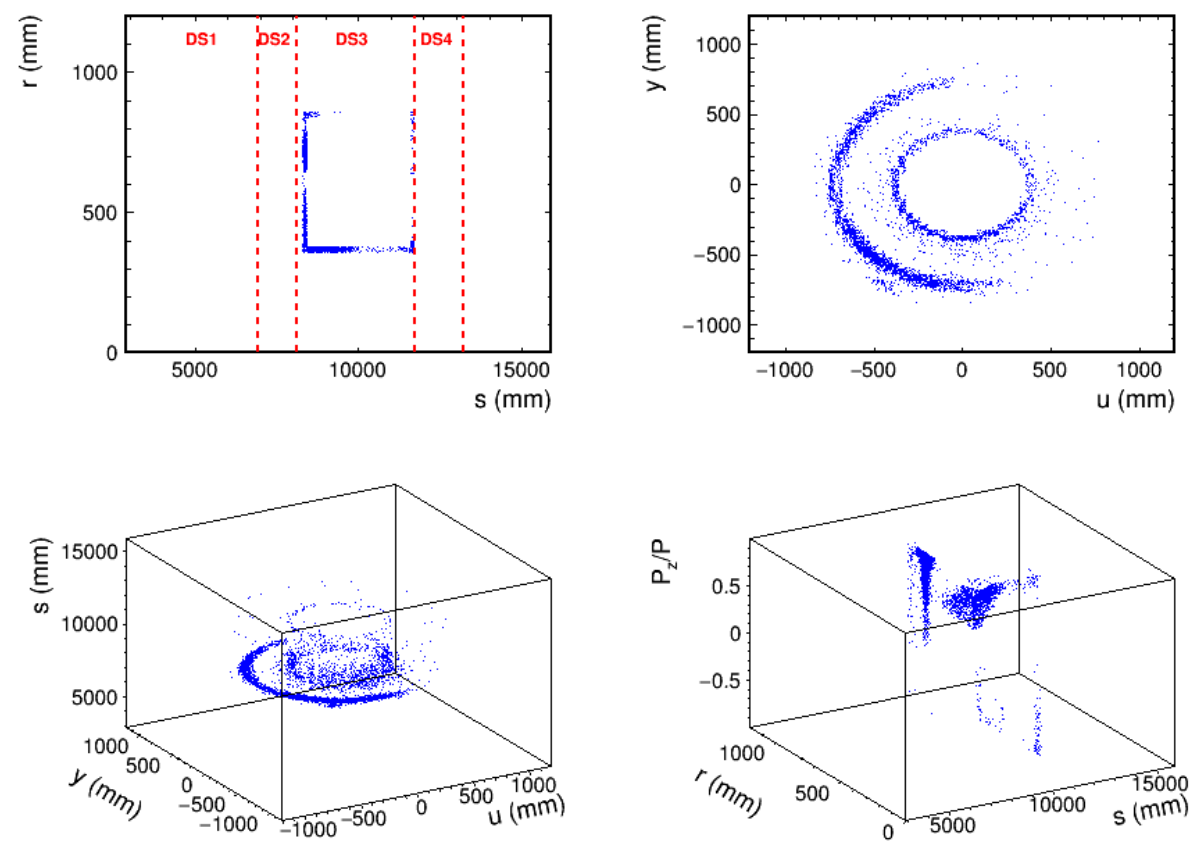

Figure 4-7: Position distribution of all beam flash electrons which hit the inclusive tracker volume, represented as scatter plots. All values are taken at the point where the electron crosses the tracker for the first time. The coordinate systems used are $(u, y, s)$ and $(r, \phi, s)$. In the upper left plot, the radial position is plotted with respect to the path length s. The dotted red lines delimitate the DS regions: the electron signals are in the tracker region DS3, and they are detected at the boundary of the tracker volume. The top right figure is the projection of the detector in the transverse plane: electron tracks are represented in the uy plane. The bottom left plot is the $3 D$ representation of the previous one, thus all the pattern of the tracker is shown. The bottom right figure shows the pitch values as function of $r$ and $s$.
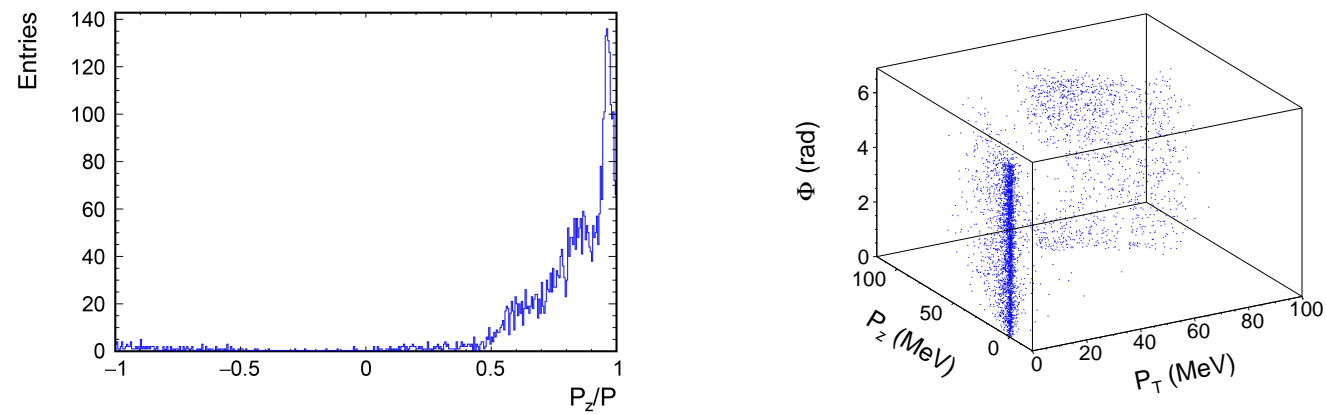

Figure 4-8: Angular distribution of beam electrons is illustrated on the left. The scatter plot on the right shows the momentum distribution in cylindrical coordinates. All values are taken at the point where electron crosses the tracker volume for the first time. Mau10 default maps are used in the simulation.

\subsection{BACKGROUND ESTIMATION ALGORITHM}

In order to select only electrons which mimic the conversion electrons, a cut on momentum and on the pitch has been applied. Following the selection criteria, the applied cuts are: 

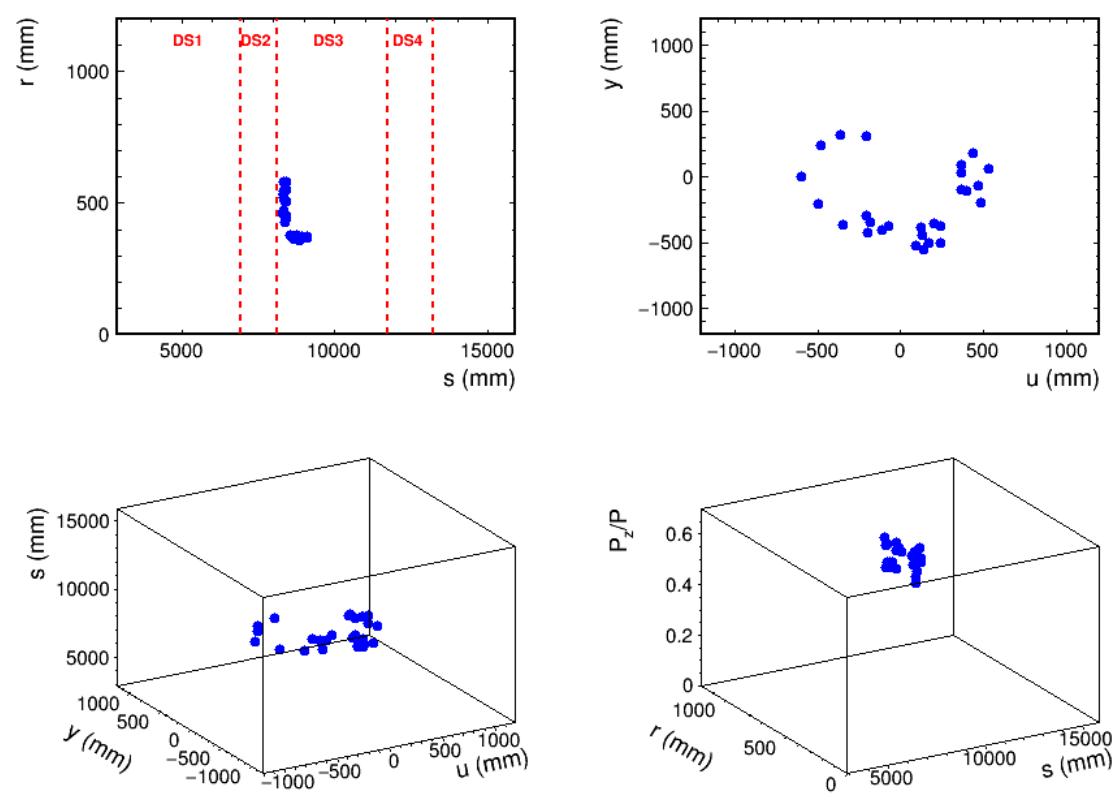

Figure 4-9: Beam flash electrons which mimic the conversion electron and reach the inclusive tracker volume. Position distribution of the points where electron crosses the inclusive tracker volume for the first time. The momentum and pitch cut of $104<p<106 \mathrm{MeV} / \mathrm{c}$ and $0.4<p_{z} / p<0.7$ are applied. The coordinate systems used are $(u, y, s)$ and $(r, \phi, s)$. In the upper left plot, the radial position is plotted with respect to the path length $s$. The dotted red lines delimitate the DS regions: the electron signals are in the tracker region DS3, and they are detected only at the entrance of the tracker. The upper right figure is the projection of the detector in the transverse plane: electron tracks are represented in the uy plane. The bottom left plot is the $3 D$ representation of the previous one, thus all the pattern of the tracker is shown. The bottom right figure shows the pitch values as function of $r$ and $s$. Mau10 default maps are used in the simulation.

- $104<p<106 \mathrm{MeV} / \mathrm{c}$;

- $0.4<p_{z} / p<0.7$.

After applying these cuts, the momentum and position distributions look very different: the momentum magnitude is just a spike, and the position distribution of crossing point looks more uniform in the XY plane (see Figure 4-9). Moreover, signal-like electrons are only detected in the entrance of the tracker, thus they are most likely not reconstructable signals.

The generated sample of incoming electrons corresponds to $N_{\text {stat }}=2.1 \times 10^{15}$ POT, where $2.1 \times 10^{9}$ is the number of single-stage simulated events and a factor of $10^{6}$ is included to account for the resampling. For this sample, $N_{e}=27$ electrons hit the tracker with $104<p<106 \mathrm{MeV} / \mathrm{c}$ and $0.4<p_{z} / p<0.7$. All these high momentum electrons arrive at the DS within $150 \mathrm{~ns}$ or earlier after their parent proton hits the production target. Thus, the delayed live gate completely eliminates the background electrons originating from in-time protons. Out-of-time protons can, however, produce beam electrons in the delayed live gate, but these are suppressed by the extinction channel. Assuming $3 \times 10^{20}$ POT for the duration of the experiment, and an extinction factor of 
$10^{-10}$ [Bartoszek et al.(2014)], the background estimation is:

$$
N_{b k g} \approx \frac{N_{e}}{N_{\text {stat }}} \times 3 \cdot 10^{20} \times 10^{-10} \times 0.5=19 \times 10^{-5}
$$

where 0.5 is the live time window, which takes into account the fact that the data recording system is live for about half time between two proton beam bunches.

This estimation algorithm for the background has been applied both for the nominal fields, using the default Mau10 maps in the two stages of the simulation, and for the varied fields.

\subsection{VALIDATION}

There was a previous estimation of this background using the same procedure, but with an earlier version of the Mu2e geometry, including the previous default field maps Mau9 (see Chapter 2). The result from this estimation was $2 \times 10^{-5}$, i.e. one order of magnitude lower than the present estimation with the default field configuration. In order to validate the present analysis, it was essential to understand the difference between the two estimations.

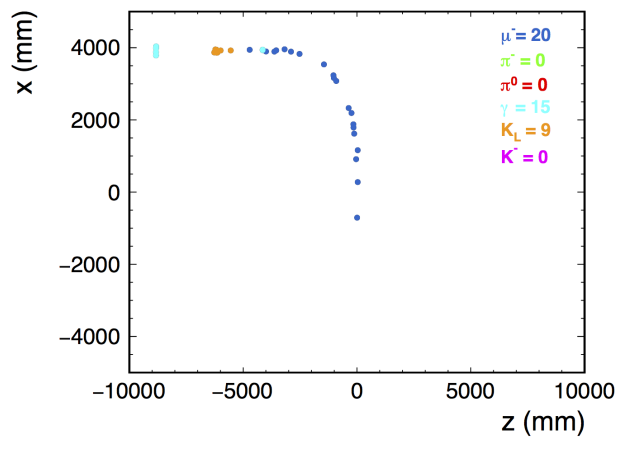

(a) Present analysis

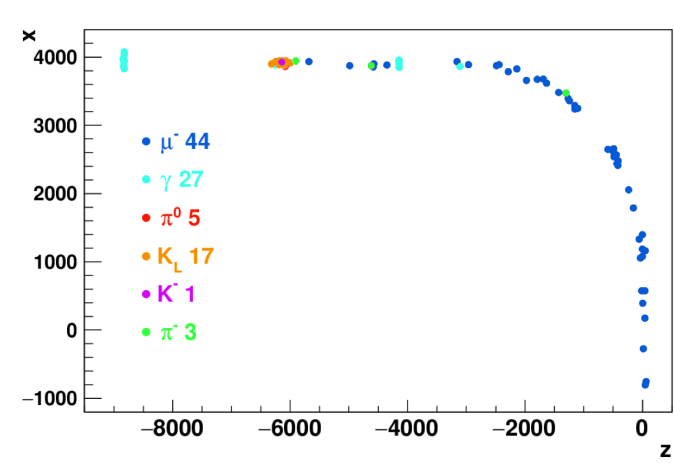

(b) Previous analysis

Figure 4-10: Locations of electron creation along the transport line up to and including the TS3 collimator in the present (4-10(a)) and previous (4-10(b)) analysis in Mu2e global coordinates. The present simulation uses Mau10 default maps, the previous one Mau9 default maps. Different colors correspond to different parent particle flavors.

Figure 4-10 compares the locations of electron creation along the transport line for the present (left window) and previous analysis (right window). The color code indicates the particle flavor of electron parents. The two results come from different statistics: the present analysis uses $2 \times 10^{9}$ events (POT), while the previous analysis uses $5 \times 10^{9}$ POT. There are 44 electrons created in the present analysis up to the TS3 collimator, as compared to 97 created in the previous analysis. Scaled to the respective simulation statistics, the present result is consistent with the previous one. However, electrons in the present analysis are created along the entire beam transport line, from 


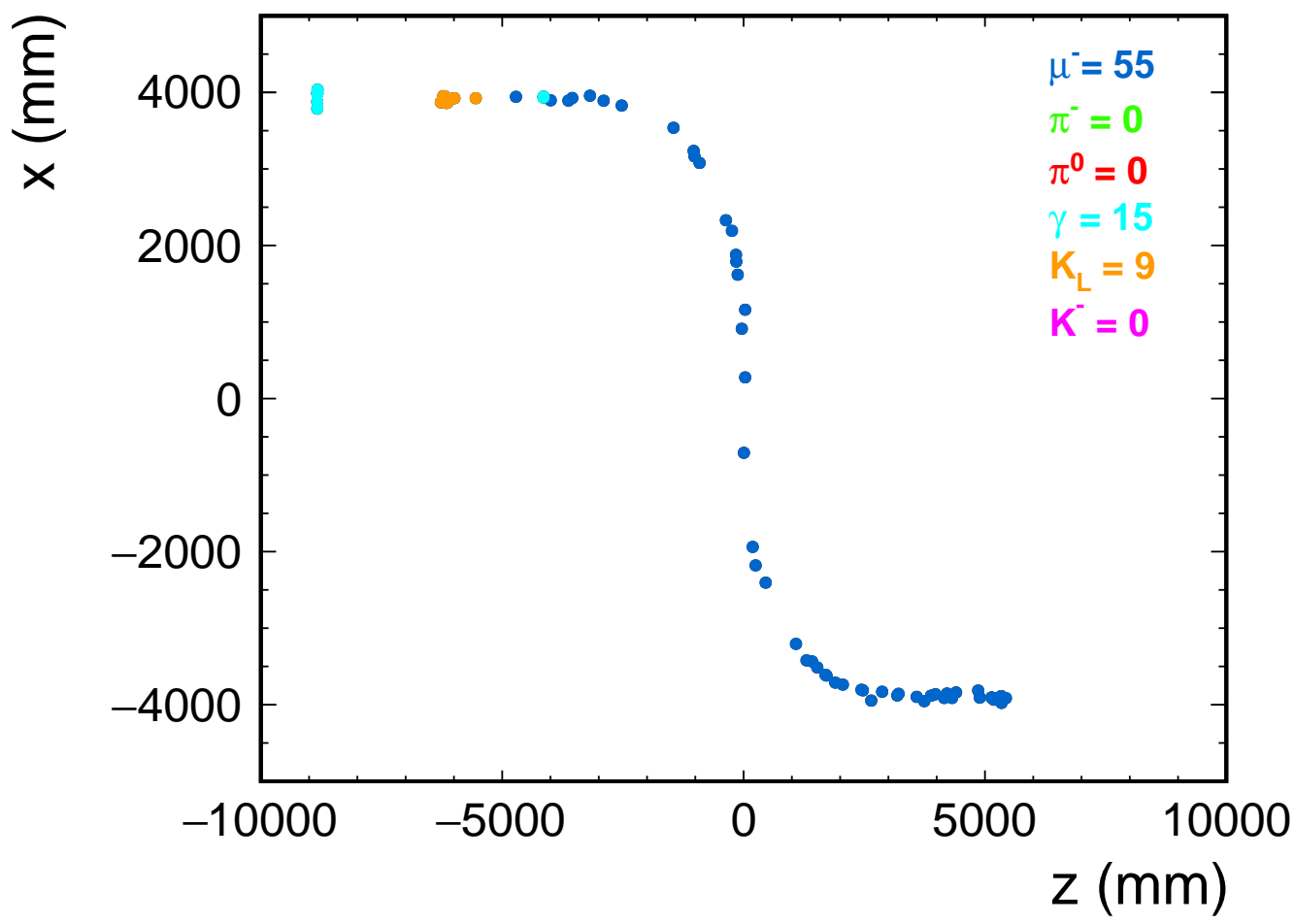

Figure 4-11: Locations of electron creation along the transport line in the present analysis in Mu2e global coordinates using Mau10 default maps. Different colors correspond to different parent particle flavors.

the production to the capture target, as shown in Figure 4-11, whereas in the previous analysis they are created only through the TS3 collimator. Therefore, in the present analysis, the total number of electrons arriving at the capture target is 79 , which is nearly a factor of two higher than the corresponding number (97) in the previous analysis, scaled to the corresponding simulation statistics.

Another difference between the present and previous analyses is that in the previous one there are 8 electrons created by pion decays, whereas in the present one there is none created by pion decays. This difference is attributed to the poor statistics of the sample. There are 20 electrons created by muons in the present analysis (44 in the previous one), 15 electrons created by photons in the present analysis (27 in the previous one) and 9 electrons created by kaons in the present analysis (18 in the previous one). These categories scale with the statistics of the two simulations. In the present analysis there 35 more electrons, all from muon decays, created in TSd and DS.

In addition to the higher beam electron rate, the difference in the electron production locations between the present and the previous analysis results in a different phase space of the electrons that arrive at the capture target. As Figure 4-12 shows, the electrons at the beginning of the capture target in the present analysis are much more spread in the $\mathrm{Y}$ dimension than the corresponding 


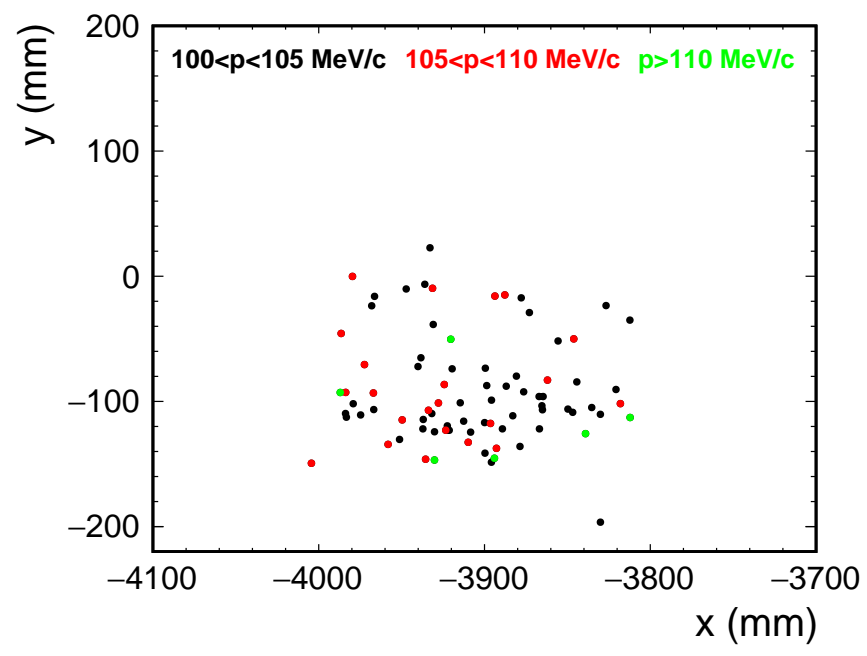

(a) Present analysis

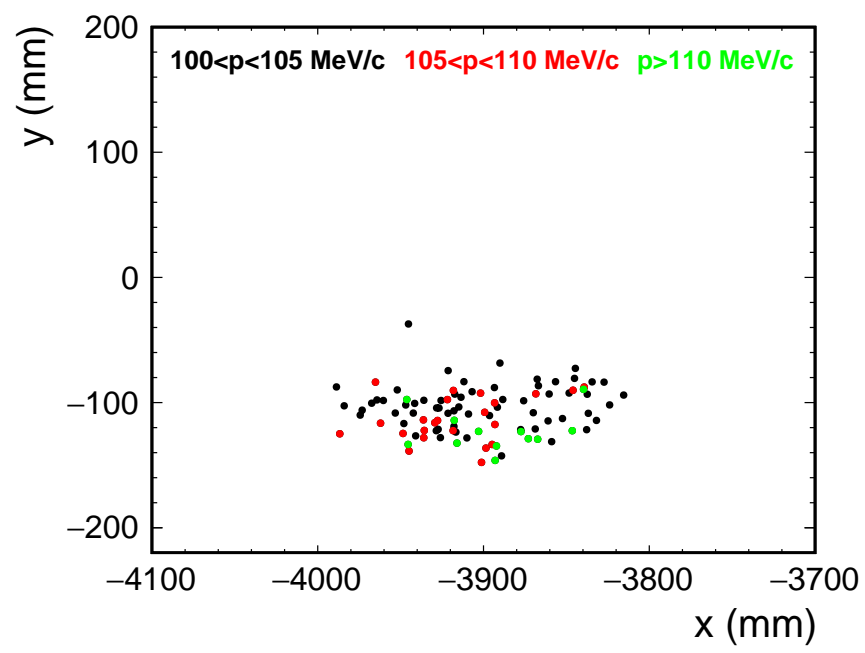

(b) Previous analysis

Figure 4-12: Distribution of electrons in Mu2e global transverse coordinates at the beginning of the capture target $(\mathrm{Z}=5871 \mathrm{~mm})$, in the present (4-10(a), Mau10 default maps) and previous (4-10(b), Mau9 default maps) analysis. Different colors correspond to different electron momentum range. Black points represent electrons with $100<p<105 \mathrm{MeV} / \mathrm{c}$, red points electrons with $105<p<110 \mathrm{MeV} / \mathrm{c}$ and green points electrons with $p>105 \mathrm{MeV} / \mathrm{c}$.

electrons in the earlier analysis. This is explained by the fact that in the present analysis a number of electrons arriving at the capture target are created in TSd or in the beginning of DS and thus they are not enough collimated and focused enough by the magnetic field. This spread affects directly the probability of the resampled electrons to scatter off some material downstream and then hit the tracker. As a result, the rate of electron hits in the tracker is much higher in the present analysis. Moreover, in the present analysis, the electrons hitting the tracker illuminate much more volume of 


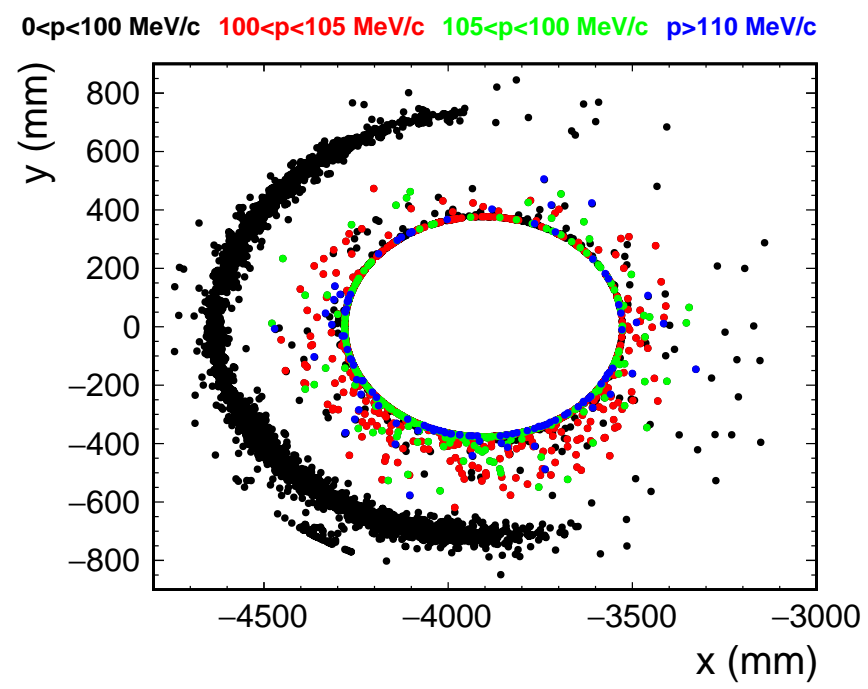

(a) Present analysis

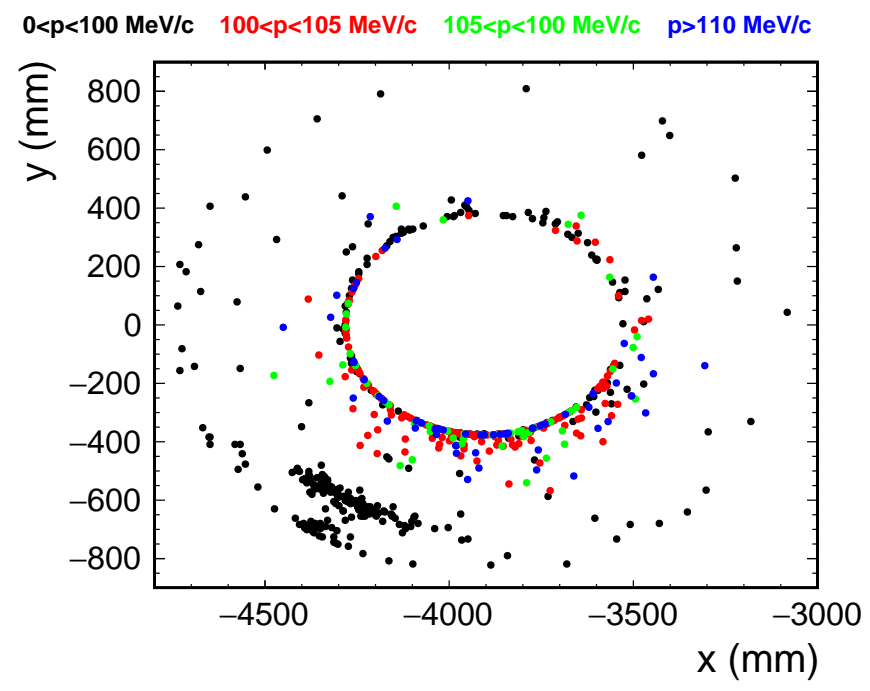

(b) Previous analysis

Figure 4-13: Distribution of electrons hitting the surrounding volume of the tracker in the Mu2e global transverse coordinates using Mau10 default maps. The plot 4-10(a) shows the distribution in the present analysis, the plot 4-13(b) the previous one. Different colors correspond to different electron momentum range. Black points represent electrons with $0<p<100 \mathrm{MeV} / \mathrm{c}$, red points electrons with $100<p<105 \mathrm{MeV} / \mathrm{c}$, green points electrons with $105<p<110 \mathrm{MeV} / \mathrm{c}$ and blue points electrons with $p>110 \mathrm{MeV} / \mathrm{c}$.

the tracker, as shown in Figure 4-13. In that figure, the ring with the larger radius corresponds to the outer wall of the tracker and the ring with the smaller radius to the inner wall. The outer wall is hit by low momentum electrons (from conversions and $\delta$ rays), whereas the inner wall is hit by high momentum electrons. As the figure shows, in the earlier analysis only a small low-X, $Y<0$ corner of the outer wall is hit, whereas in the present analysis all of the low-X side of the outer wall, for any $\mathrm{Y}$, is hit by low momentum electrons. 


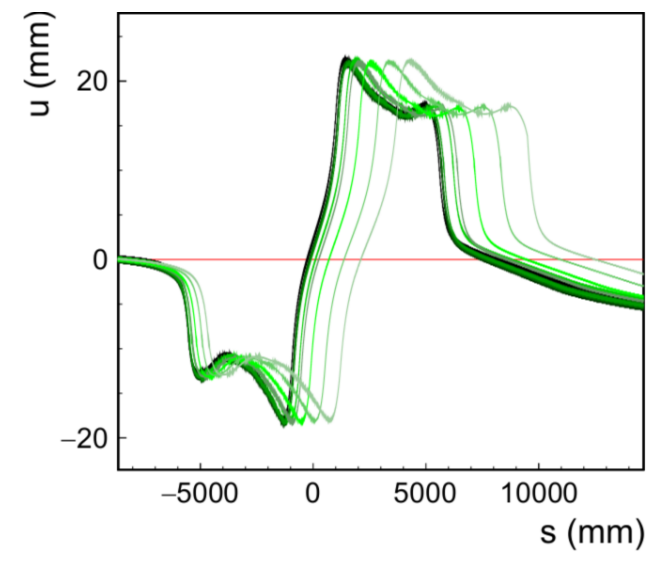

(a) Horizontal plane

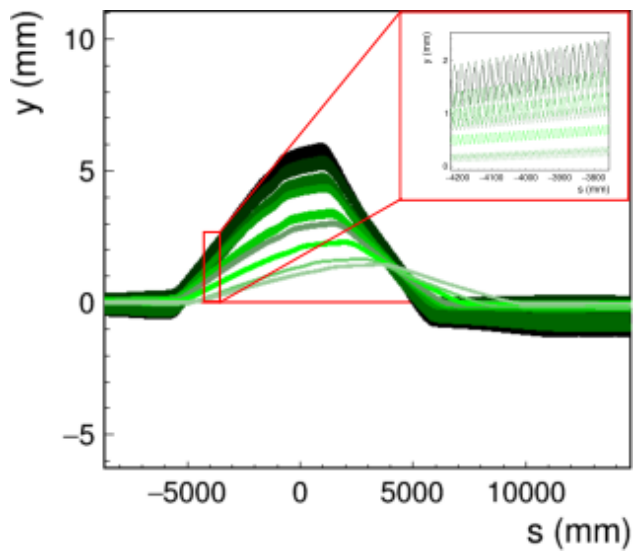

(b) Vertical plane

Figure 4-14: Electron trajectories in the magnetic field without misalignments, in the local coordinate system $(u, y, s)$ defined in subsection 1.3.1. The left plot (4-14(a)) shows the trajectories in the horizontal plane and the right plot (4-14(b)) the trajectories in the vertical plane. The range of momenta is $0.4<p<2.4 \mathrm{MeV} / \mathrm{c}$ (see Chapter 5). Darker green color corresponds to higher momentum. The vertical displacement upwards and back due to the S-shape of the Transport Solenoid is manifest. In the right plot $(4-14(b))$, the inset shows a magnification of the selected region, marked with the red box. The magnification shows the helical motion of the electrons around the field lines.

Figure 4-12 shows that the electrons arriving at the capture target are mostly distributed at $Y<Y_{0}$ and $X<X_{0}$, where $\left\{X_{0}, Y_{0}\right\}=\{-3904,0\} \mathrm{mm}$ are the transverse coordinates of the DS axis. This displacement from the axis is explained in Figure 4-14, where low momentum electrons are traced in the magnetic field without misalignment (see Chapter 5). Because of the small Larmor radius of their motion, corresponding to their small transverse momentum, these electrons spiral closely around the magnetic field lines and, on a large scale, they approximately describe them. As shown in the figure, the electron trajectories, and therefore the field lines, are all displaced towards $Y<Y_{0}$ and $X<X_{0}$ in DS, which extends in $s>6576 \mathrm{~mm}$. It is thus the field itself that displaces the particles towards $Y<Y_{0}$ and $X<X_{0}$.

\subsection{Results}

Table 4.2 summarizes the results of beam electron simulations for various field configurations. All simulations are preformed following the same algorithm described in section 4.1. The first row shows the results of the previous analysis, where the simulation was done with different geometry and different field than the current ones. The next two rows show the results of the present analysis, using the field of the previous analysis (Mau9, second row) and the field of the present analysis (Mau10, third row), without rotation in both cases. The following two rows contain the results of simulations using the Mau10 design with TS rotated by $0.15^{\circ}$ (fourth row) and $0.50^{\circ}$ (fifth row) about the $-Z$ axis. The following eight rows contain the results of simulations using the Mau9 
design with TS rotated as described in the respective entries in the first column of the table. Rows six through nine contain results of simulations with rotations of $0.1^{\circ}$ and rows ten though thirteen contain results of simulations with rotations of $1^{\circ}$. No shifted coil simulations were performed, since the effects of this type of misalignments have been consistently found to be negligible.

\begin{tabular}{|c|c|c|c|c|c|}
\hline \multirow{2}{*}{ Maps } & \multirow{2}{*}{$\begin{array}{c}\mathbf{N}_{\text {stat }} \\
\left(\times 10^{15}\right)\end{array}$} & \multirow{2}{*}{$\begin{array}{c}e^{-} \text {at VD9 } \\
\text { BEFORE resampling }\end{array}$} & \multicolumn{2}{|c|}{$e^{-}$in the tracker } & \multirow{2}{*}{$\begin{array}{r}\mathbf{N}_{\mathrm{bkg}} \\
\left(\times 10^{-5}\right) \\
\end{array}$} \\
\hline & & & BEFORE cuts & AFTER cuts $\left(\mathbf{N}_{\mathbf{e}}\right)$ & \\
\hline Previous Estimation (Mau9) & 5.0 & 97 & 632 & 7 & 2 \\
\hline SolCalc (Mau9) DeFault & 2.0 & 50 & 4504 & 17 & 13 \\
\hline MaU10 DEFAUlt & 2.1 & 81 & 4106 & 27 & 19 \\
\hline MAU10 $0.15^{\circ}$ wrt $-Z$ TSu, $0.15^{\circ}$ wrt $-Z$ TSd & 2.0 & 89 & 3841 & 18 & 14 \\
\hline MAU10 $0.50^{\circ}$ wrt $-Z$ TSu, $0.50^{\circ}$ wrt $-Z$ TSd & 1.9 & 133 & 3010 & 22 & 17 \\
\hline SolCalc (MaU9) $0.1^{\circ} \mathrm{wrt}+X \mathrm{TSu}, 0.1^{\circ} \mathrm{wrt}+X \mathrm{TSd}$ & 1.8 & 63 & 5204 & 34 & 28 \\
\hline SolCalc (Mau9) $0.1^{\circ}$ wrt $-X$ TSu, $0.1^{\circ}$ wrt $-X$ TSd & 2.0 & 63 & 3255 & 23 & 17 \\
\hline SolCalc (MaU9) $0.1^{\circ}$ wrt $+X$ TSu, $0.1^{\circ}$ wrt $-X$ TSd & 2.0 & 61 & 5771 & 36 & 27 \\
\hline SolCalc (MaU9) $0.1^{\circ} \mathrm{wrt}-X \mathrm{TSu}, 0.1^{\circ} \mathrm{wrt}+X \mathrm{TSd}$ & 2.0 & 77 & 4364 & 36 & 27 \\
\hline SolCalc (Mau9) $1^{\circ}$ wrt $-X$ TSu, $1^{\circ}$ wrt $+X$ TSd & 2.0 & 4 & $\mathrm{~N} / \mathrm{A}$ & $\mathrm{N} / \mathrm{A}$ & $\mathrm{N} / \mathrm{A}$ \\
\hline SolCalc (Mau9) $1^{\circ}$ wrt $+X$ TSu, $1^{\circ}$ wrt $-X$ TSd & 1.8 & 387 & 26575 & 216 & 180 \\
\hline SolCalc (Mau9) $1^{\circ}$ wrt $+X$ TSu, $1^{\circ}$ wrt $+X$ TSd & 1.8 & 14 & 1029 & 3 & 2 \\
\hline SolCalc (Mau9) $1^{\circ}$ wrt $-X$ TSu, $1^{\circ}$ wrt $-X$ TSd & 2.0 & 191 & 4926 & 55 & 42 \\
\hline
\end{tabular}

Table 4.2: Summary of estimates of the beam-electron induced background yield for various field configurations, including TS misalignments. The notation for the maps has been defined in 2.5 .

In the overall, all numbers scale with the rotation, almost linearly within statistics. The extreme case of $1^{\circ}$ rotation of TSu about the $-X$ axis and $1^{\circ}$ rotation of TSd about the $+X$ axis gives only 4 electrons at the beginning of the capture target. In this case, no resampling is possible due to the lack of electrons at the target (marked as "resampling not applicable", N/A, in the table), so the result is considered as the beam electron background being consistent with zero in this case. The opposite extreme case is the rotation of $1^{\circ}$ of TSu about the $+X$ axis and $1^{\circ}$ rotation of TSd about the $-X$. This is the inverse rotation of the previous extreme case. In this case the background yield from beam electrons is maximum, reaching a value of $180 \times 10^{-5}$. The results of all other rotations fall in between these two extremes.

As stated previously, the $1^{\circ}$ rotations are exaggerations of possible TS misalignments. More realistic, i.e. more likely to happen, are the $0.1^{\circ}$ rotations. The background yields of the simulations assuming the $0.1^{\circ}$ rotations cluster around $2.5 \times 10^{-4}$ with a dispersion of about $1.2 \times 10^{-4}$. Taking conservatively this number as an average estimate of the background in account of misalignments, the final estimation is $(2.5 \pm 1.2) \times 10^{-4}$ or $<5 \times 10^{-4}$ at $90 \%$ C.L. This number is one order of magnitude higher than the previous estimate of $<5 \times 10^{-5}$, but implies that this remains one of the smallest backgrounds of the experiment. 


\section{Chapter 5}

\section{TESTS OF THE TRANSPORT SOLENOID FIELD}

\section{WITH LOW MOMENTUM ELECTRONS}

\subsection{THE BETA SOURCE TEST}

\subsubsection{Motivation}

After the solenoid system commissioning will be completed, but before installation of shielding around the solenoids takes place, the magnetic field of the solenoids has to be mapped. While the field of PS and DS solenoids will be measured using field probes, the same is not possible for TS because of the complicated design of the support system, interfering mechanically with the magnetic coils. An alternative possible solution, proposed in the Mu2e Field Mapping System ([Mu2e Collaboration(2015)], version 1.3 of 6/9/2014) is to use a commercial source of charged particles (electrons) emitted into the TS field. This will be a test of low energy electron transport from the PS target, through the TS1, TS3, and TS5 collimators, to the DS stopping target region. It is planned to occur in two stages. First, a detector placed near the exit of TSu will measure the transverse position where electrons traverse the TS3u collimator, as a function of the starting transverse position of a source at the production target location. Second, this detector will be removed and placed near the exit of TSd to measure the transverse position where the electrons arrive, as a function of their starting position. For this test to occur, the $\bar{p}$ absorption windows must not be installed. 


\subsubsection{Description of the test}

The idea of the $\beta$ source test is to use a narrow beam of electrons emitted by a low-energy $\beta$ source located in PS, and to trace them using a detector located at various positions along the transport line. The detector should have a sufficient segmentation, so that the field uniformity can be tested by shifting the beam axis to various $X-Y$ positions relative to the solenoid axis.

The principle of the test is that Lorentz forces drive electrons with initial momentum along the field lines to travel though the TS field. Low momentum electrons follow paths very close to the field lines, and thus they can trace the field if they are detected at various positions inside the solenoids. As described in section 1.2, the path of the electrons in the curved magnetic field of TS is helical: even if the initial electron velocity is parallel to the magnetic field (i.e. no forces are present), the curvature of the field will give rise to Lorentz forces that will cause the electron to follow a helical path (at constant kinetic energy) with a circular component in a plane perpendicular to the field line and a drift component along the field line. For $B \sim 2 \mathrm{~T}$ and $\sim 1 \mathrm{MeV} / \mathrm{c}$ electron momentum, the Larmor radius is of the order of $2 \mathrm{~mm}$, which gives the figure of merit for the dispersion of an electron beam originating from a collimated source. Because of the helical motion in the curved TS field, the actual electron path lengths are much larger (15-20 times on the average) than the distance along the solenoid axis from the source to the detector.

The source and the detector require some infrastructure for mechanical support, alignment and cabling for the detector (power and data readout). For this infrastructure to fit in the design of the solenoids, some space is needed, which will therefore not allow for the transport line to be sealed and the magnets to be cooled down to superconducting state. Without sealing, only a moderate vacuum inside the transport line can be achieved. This implies medium effects on the electron paths across the transport line. The results of these effects can be mitigated by either filling the transport line with helium or reducing the air pressure to a sufficiently low value.

\subsubsection{Medium effects}

The finite pressure in the transport line causes the electrons to undergo multiple scattering off the atoms of the air, thus losing some energy while traversing the distance from the source to the detector.

When an electron penetrates the air, it will interact with the atoms present in the medium through the electromagnetic force. There are two main effects: energy loss and deflection. The energy loss amounts to energy transferred from the electron to the atoms of the medium without changing significantly the direction of motion of the electron. The deflection amounts to the change in the direction of the motion of the electron due to multiple consecutive interactions of the electron with atoms of the medium. 
Fast electrons lose energy by exciting or ionizing atoms along their trajectory (straggling effect). In a single interaction, by depositing energy to the atom larger than the binding energy of an atomic electron, that atomic electron will be released from the atom, producing what is called a $\delta$ ray. If the energy deposit is below the binding energy, then the atom is exited and subsequently de-exited by the emission of a soft $\gamma$ ray. When this process is repeated many times, the sum of energies lost by the electron to the ionized or exited atoms amount to what is called total ionization loss [Landau(1944), Vavilov(1957)].

When the energy lost by the electron in a single interaction with an atom in the medium is sufficiently large, then the electron can emit a photon $(\gamma$ ray $)$ that carries away part of the lost energy, while the rest is transferred to the atom. This mechanism is known as bremsstrahlung and results in the so-called radiative energy loss [hei(1954)]. Both straggling and bremsstrahlung contribute to the total energy loss of the electrons in the medium.

The second main effect of the interaction of the electrons with matter is the multiple deflection. Traversing the medium, the electrons are deflected by many small-angle Coulomb scatters, and hence the effect is called multiple Coulomb scattering. The electrons randomly change their direction, thus the spread of the initial electron beam increases. The Coulomb scattering distribution is well described by the Molière theory [Bethe(1953)], which can be accurately approximated by a Gaussian distribution for small deflection angles.

The intensity of background particles ( $\delta$ and $\gamma$ rays) produced by electron interaction with matter scales with the air density or pressure. Therefore, the electron paths have to be traced in a model of the TS field with a realistic simulation program (GEANT4), accounting for the phase space of the electron beam.

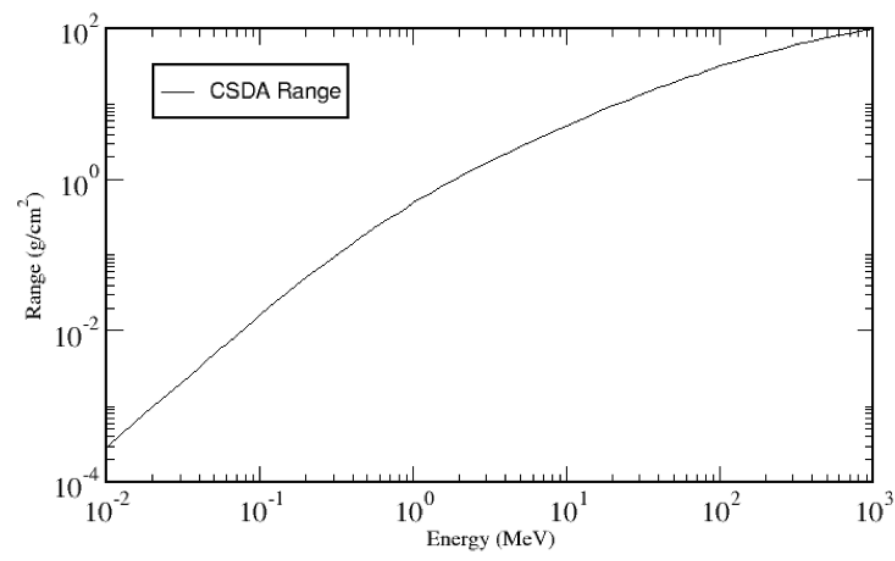

\begin{tabular}{c||c}
\hline \hline $\begin{array}{c}\text { Electron ENERGY } \\
(\mathrm{MeV})\end{array}$ & $\begin{array}{c}\text { RanGE } \\
(\mathrm{m})\end{array}$ \\
\hline 0.1 & 0.1 \\
0.2 & 0.4 \\
0.3 & 0.8 \\
0.5 & 1.7 \\
0.7 & 2.6 \\
1 & 4.1 \\
1.25 & 5.3 \\
1.5 & 6.6 \\
1.75 & 7.8 \\
2 & 9 \\
\hline \hline
\end{tabular}

Figure 5-1: Electron range in STP air calculated in the Continuous Slowing Down Approximation (CSDA). 
Figure 5-1 shows the electron range calculated in the Continuous Slowing Down Approximation (CSDA) [CSDA(2015)], with an air density of $0.0012 \mathrm{~g} / \mathrm{cm}^{3}$ in Standard Temperature and Pressure (STP). The range scales inversely with the air density, so the range for 1-2 $\mathrm{MeV}$ electrons is 4-9 $\mathrm{m}$ in atmospheric air. Electrons with energies much higher than those provided by $\beta$ sources would be needed to make it though the TS solenoid in standard air pressure. Thus, low air pressure inside TS is mandatory. Another possibility is to fill the transport line with helium. This element, due to its low atomic number $Z=2$, would reduce the strength of interactions with the beam electrons and consequently would reduce energy loss and multiple deflection effects.

\subsubsection{Source model}

The electron source has to be chosen on the basis of clear requirements: sufficient momentum range, moderate source activity (to suppress hazard) and lack of background particles (e.g. photons). There are several source options: a cathode ray tube, an internal conversion source or a $\beta$ source. $\mathrm{A}$ cathode is simple and easy to use but has very low energy. An internal conversion source, such as ${ }^{125} \mathrm{I}$ or ${ }^{99 m} \mathrm{Tc}$, is always associated with electron capture that produces $\gamma$-rays. It has the advantage of a narrow energy spectrum, because the kinetic energy range of conversion electrons is close to the atomic K-shell energy from which the electron originates, but lower energy than $\beta$ source (capture occurs below $\beta$ decay threshold), lots of backgrounds from Auger electrons and $\delta$-rays and short life-time, which means high activity and treatment as a hazardous material.

Table 5.1: High energy $\beta$ sources

\begin{tabular}{ccccc}
\hline \hline Isotope & Activity $\left(\mu \mathbf{C i}^{*}\right)$ & Half-life (y) & Emissions & Decay energy (keV) \\
\hline${ }^{137}$ Cs S. & 0.2 & 30.17 & $94.6 \%(\beta, \gamma), 5.4 \% \beta$ & $(\beta 512, \gamma 662), \beta 1173$ \\
${ }^{137}$ Cs S.5 & 0.5 & 30.17 & $94.6 \%(\beta, \gamma), 5.4 \% \beta$ & $(\beta 512, \gamma 662), \beta 1173$ \\
${ }^{137}$ Cs S1 & 1 & 30.17 & $94.6 \%(\beta, \gamma), 5.4 \% \beta$ & $(\beta 512, \gamma 662), \beta 1173$ \\
${ }^{137}$ Cs S5 & 5 & 30.17 & $94.6 \%(\beta, \gamma), 5.4 \% \beta$ & $(\beta 512, \gamma 662), \beta 1173$ \\
${ }^{90} \mathrm{Sr} /{ }^{90} \mathrm{Y}$ & 0.1 & 28.79 & $100 \%(\beta, \beta)$ & $(\beta 546, \beta 2280)$ \\
\hline \hline
\end{tabular}

Another possibility is to use a $\beta$ decay source, such as ${ }^{137} \mathrm{Cs},{ }^{90} \mathrm{Sr} /{ }^{90} \mathrm{Y}$ or ${ }^{106} \mathrm{Ru}$ : these are best options for their high energy, but have a broad electron energy range. As shown in Table 5.1, the ${ }^{137}$ Cs decays to ${ }^{137} \mathrm{Ba}$ either through cascade $\beta^{-}+\gamma$ emission or through direct $\beta^{-}$emission, but since the cascade mode is predominant, the CNS Survey classifies it as a low-energy $\beta$ source. The high-activity versions compensate for the low rate of the direct $\beta$ decay mode, at the cost of backgrounds from the cascade decay mode. Naturally occurring strontium is nonradioactive and nontoxic at levels normally found in the environment, but ${ }^{90} \mathrm{Sr}$ is a radiation hazard. ${ }^{90} \mathrm{Sr}$ undergoes

\footnotetext{
${ }^{*} 1 \mu \mathrm{Ci}=37,000$ decays $/ \mathrm{s}$
} 
$\beta^{-}$decay with a half-life of 28.79 years and a decay energy of $0.546 \mathrm{MeV}$ distributed to an electron, an anti-neutrino, and the yttrium isotope ${ }^{90} \mathrm{Y}$. This, in turn, undergoes $\beta^{-}$decay with half-life of 64 hours and decay energy $2.28 \mathrm{MeV}$ distributed to an electron, an anti-neutrino, and ${ }^{90} \mathrm{Zr}$, which is stable. ${ }^{90} \mathrm{Sr} /{ }^{90} \mathrm{Y}$ is almost a pure beta particle source (Figure 5-2); the gamma photon emission from the decay of ${ }^{90} \mathrm{Y}$ is so infrequent that it can normally be ignored.

Because of higher energy, lower activity and lack of background, the ${ }^{90} \mathrm{Sr} /{ }^{90} \mathrm{Y}$ is the best option among the $\beta$ source, but a collimation system subtending a solid angle of $\mathrm{O}(10 \mathrm{msr})$ is needed to make a beam out of the emitted electrons, retaining an appreciable flux well above cosmic ray background. In the simulation the $\beta$ source is modeled as a narrow beam of electrons emitted within a cone of $8^{\circ}$ half-aperture $\left(0^{\circ} \leq \theta \leq 8^{\circ}\right.$ with respect to the $\mathrm{Z}$ axis). This collimation allows for an appreciable $\beta$ emission rate of $\sim 10$ electrons/s, which corresponds to $\sim 1$ hour/shot for $\sim 50 \mathrm{k}$ electrons/shot.

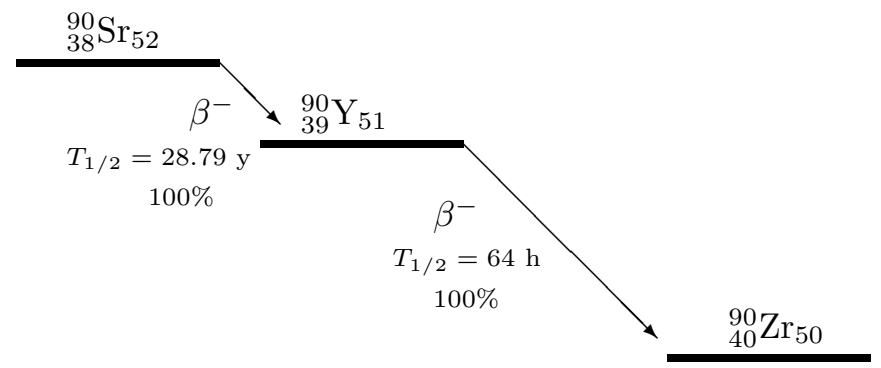

Figure 5-2: $\beta^{-}$decay of the ${ }^{90}$ Sr into ${ }^{90} Y$, which decays into an electron and ${ }^{90} \mathrm{Zr}$.

The ${ }^{90} \mathrm{Sr} /{ }^{90} \mathrm{Y}$ source spectrum is given by the $\beta$-decay transition probability obtained by using the point-nucleus Fermi function ([Logue and Chern(1968)]). From the Fermi theory of $\beta$ decay, the shape of the energy distribution is given approximately by the expression:

$$
N(p)=C p^{2}\left(Q-\sqrt{p^{2} c^{2}+m_{e}^{2} c^{4}}+m_{e} c^{2}\right)^{2} F(Z, p)
$$

where $p$ is the momentum of the electron and $Q$ represents the energy yield of the transition. $F(Z, p)$ is called the Fermi function: it accounts for the nuclear Coulomb interaction between the $\beta$ particle and the daughter nucleus, which shifts this distribution toward lower energies. $Z$ is the atomic number of the daughter nucleus and $C$ is a normalization constant [Krane and Halliday(1987)].

In a first-order perturbation calculation in $Z \alpha$, the Fermi function is given by:

$$
F(Z, p)=1+\pi Z \alpha \frac{\sqrt{p^{2}+m_{e}^{2} c^{2}}}{p}
$$

This perturbative calculation includes electromagnetic radiative corrections, finite-nuclear size and screening of the nuclear Coulomb field by the atomic electrons. 5-3(a) shows the modeled spectra for ${ }^{90} \mathrm{Sr},{ }^{90} \mathrm{Y}$ and ${ }^{90} \mathrm{Sr} /{ }^{90} \mathrm{Y}$, while $5-3(\mathrm{~b})$ is the sum of the ${ }^{90} \mathrm{Sr}$ and ${ }^{90} \mathrm{Y}$ spectra produced in 
the simulation: the first peak corresponds to ${ }^{90} \mathrm{Sr}$, the second one to ${ }^{90} \mathrm{Y}$.

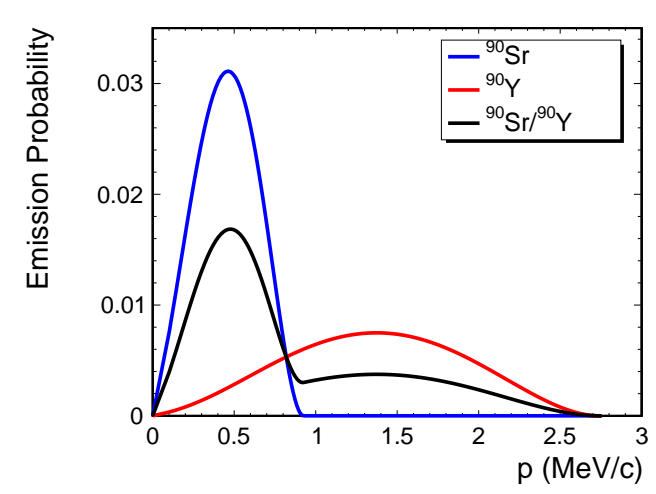

(a)

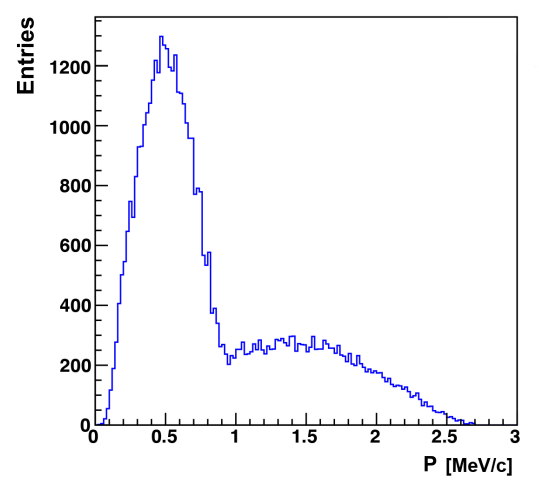

(b)

Figure 5-3: Momentum spectra of emission probabilities. The left figure (5-3(a)) shows the theoretical model spectra for ${ }^{90} \mathrm{Sr},{ }^{90} \mathrm{Y}$ and ${ }^{90} \mathrm{Sr} /{ }^{90} \mathrm{Y}$ : the ${ }^{90} \mathrm{Sr}$ is in red, the ${ }^{90} \mathrm{Y}$ is blue, while the black line is the sum of the two spectra. The curves are normalized. The histogram on the right $(5-3(b))$ is the sum of the ${ }^{90} \mathrm{Sr}$ and ${ }^{90} Y$ spectra as results of the simulation (Mau10 default).

\subsubsection{Detector model}

The choice of the detector is driven by the precision requirements of the test, that are high resolution, high efficiency, uniform response and immunity to magnetic field.

A possible solution could be a scintillating fiber tracker, which is a well established technology [Ruchti(1996)].. The most common geometry for a scintillating fiber tracker is the so-called active target geometry, which can be formed from coherent arrays of scintillating glass fibers, plastic fibers, or capillaries filled with liquid. The targets typically contain $10^{5}-10^{6}$ individual detection elements, with optically precise end faces. Often, one end face is coated with a reflective material such as aluminum; the other face is contact-coupled to an image intensifier with a fiber-optic entrance window. As ionizing radiation penetrates into or through the scintillation material, a portion of the energy loss results in the emission of scintillating light. A fraction of this light (typically a few percent) is trapped by total internal reflection inside the fibers and is piped (a) toward the readout end, where it is detected at the photocathode of the image intensifier; and (b) toward the mirrored end, where a portion $(70-80 \%)$ is reflected back toward the readout end and subsequently detected. The incident particle beam can be directed in any angle relative to the orientation of the fibers that comprise the target. Examples include transverse and head-on orientations.

This type of detector is immune to magnetic field and has a good spatial resolution, down to $\sim 100 \mu \mathrm{m}$ or better, and high efficiency of nearly $100 \%$. The tracker plane should have a small thickness and double layers of scintillating fibers at an angle, in order to provide $\mathrm{X}$ and 
Y measurements. The minimum kinetic energy of an electron that such a detector could detect, assuming silicon fibers, is of $\sim 40 \mathrm{keV} / \mathrm{c}$, which translates to a momentum threshold of $\sim 200 \mathrm{keV} / \mathrm{c}$ [CSDA(2015)].

In the simulation eight virtual detectors (VD) are used at the positions shown in Figure 5-4. A virtual detector is an idealized approximation of a real detector. Each pair of virtual detectors surrounds one collimator window. There are 2 virtual detectors in the entrance of TS1 and 4 virtual detectors in TS3 enclosing the 2 collimator windows: TS3u (upstream), TS3d (downstream). The last 2 virtual detectors enclose the last collimator TS5. The virtual detectors are ideal infinite detectors, which span all the transverse plane. A spatial detector resolution is folded in the simulation assuming a $\sigma=300 \mu \mathrm{m}$, based on a fiber tracker prototype developed by the Northern Illinois University (NIU) [Coutrakon et al.(2014)]. A threshold of $200 \mathrm{keV} / \mathrm{c}$ is used in the simulation for the momentum sampling.

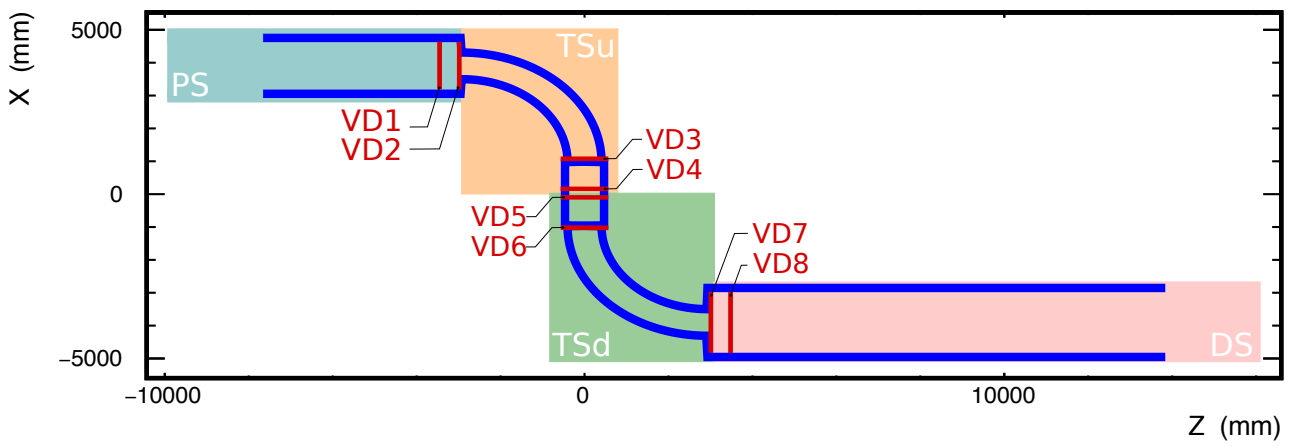

Figure 5-4: Plan view of the Mu2e solenoids and field maps, with the locations of the VD. Each pair of VD surrounds one collimator window: collimator 1 is located between VD1 and VD2, the first collimator 3 between VD3 and VD4, the second collimator 3 between VD5 and VD6, and collimator 5 is surrounded by VD 7 and VD 8. The colored blocks stand for the field maps used in the simulation.

\subsection{BetA SOURCE SIMULATIONS}

\subsubsection{Simulation parameters}

The $\beta^{-}$source test is simulated using the standard Mu2e Offline software, in the art framework which includes GEANT4 (see section 1.5). 50,000 electrons are generated by the ${ }^{90} \mathrm{Sr} /{ }^{90} \mathrm{Y}$ isotope source, modeled as point source. The angles of the electrons are sampled uniformly in the range of $0^{\circ} \leq \theta \leq 8^{\circ}$ and $0^{\circ} \leq \Phi \leq 360^{\circ}$ about the $\mathrm{Z}$ axis. 
Geometry modules are modified for each simulation to specify the magnetic field maps, the pressure and source spectrum and location. The effect of PS/TS and DS/TS misalignments on the electron motion along the transport line is studied using the varied field maps (SolCalc and Mau, see section 2.5). The vacuum pressure is set to 1 Torr for the general study, so that multiple scattering processes are allowed, but effects of the traversed material are studied separately by modifying the pressure to 0.05 Torr and 50 Torr. Both primary electrons ( $\beta$ rays) and secondary electrons ( $\delta$ rays from scattering) are recorded in the simulation using virtual detectors. $\gamma$ rays are excluded, assuming a tracking detector that can only detect charged particles. The simulation traces the path of the electrons only along TS. To maintain statistically meaningful results throughout the transport line, mean values and their errors are computed only when there are at least 50 electrons recorded at VD8.

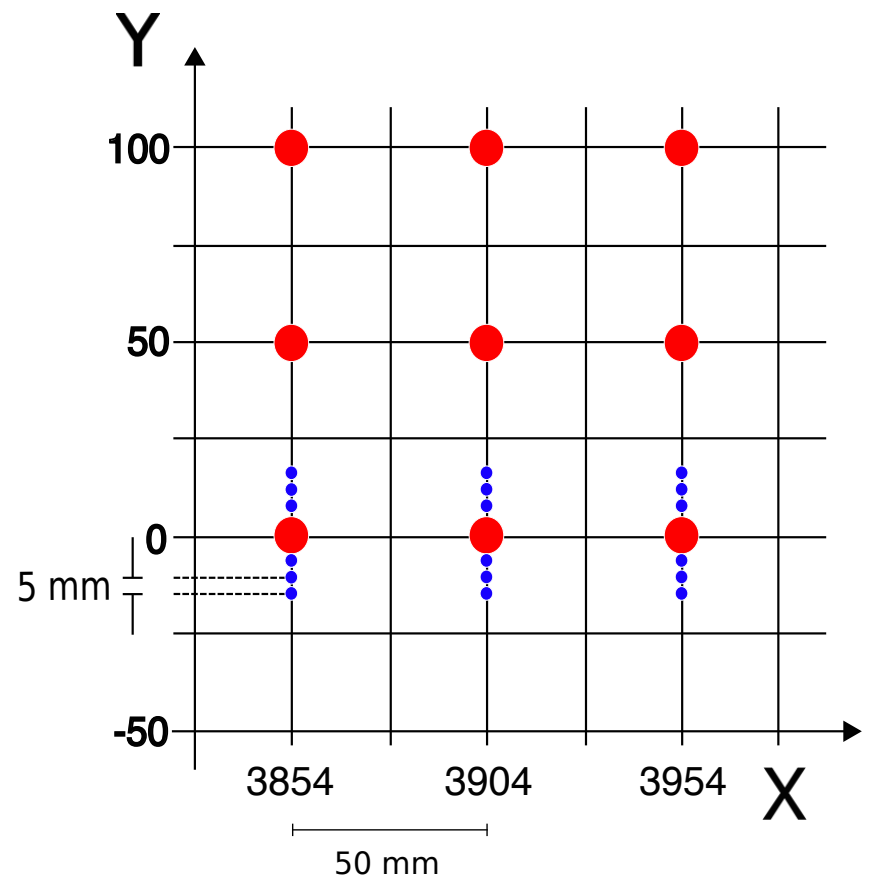

Figure 5-5: $\beta$ source locations in the $x y$ plane of the local cartesian system, relative to the solenoid axis. The points marked with red are spaced in steps of $50 \mathrm{~mm}$ above the XZ plane. The blue points are scanned in smaller steps $(5 \mathrm{~mm})$.

\subsubsection{Outline of the study}

The source is shifted to various $\mathrm{X}-\mathrm{Y}$ positions relative to the solenoid axis in a local cartesian system with the $\mathrm{z}$ axis along the solenoid axis. The $\mathrm{z}$ position of the source is fixed at $-6000 \mathrm{~mm}$, in front of the production target, which is located at $-6165 \mathrm{~mm}$. The $\mathrm{Y}$ coordinate changes from $-15 \mathrm{~mm}$ to $+15 \mathrm{~mm}$ in steps of $5 \mathrm{~mm}$ and from 50 to $100 \mathrm{~mm}$ in steps of $50 \mathrm{~mm}$. The $X$ coordinate goes 
from $-50 \mathrm{~mm}$ to $50 \mathrm{~mm}$ in steps of $50 \mathrm{~mm}$. The choice of the steps is driven by the radius of the collimators, in particular of the TS3 collimator which allows particles to go though only above the $\mathrm{XZ}$ plane. Therefore, the scan is made upwards and sideways, in steps matching the radius of that collimator. In addition, some small steps downwards and upwards are taken to scan the edge of the collimator. Figure 5-5 shows the source locations scanned in the simulation: all points of the grid have been scanned, but only the $\beta$ source locations marked with red (50 $\mathrm{mm}$ steps) and blue points (5 $\mathrm{mm}$ steps) give any electrons passing the TS3 collimator.

The expected electron hit distribution in the detector of the test can be represented by scatter plots, like those shown in Figure 5-6. The objective is to detect shifts of the centroids of these distributions, caused by a TS misalignment. The figure shows the overall shifts of these distributions that result from TS misaligned by $-0.50^{\circ}$ about the $\mathrm{Z}$ axis (Mu2e coordinate system), for the source located at various positions in the XY plane. The shifts provide an example of the expected effects that are examined in this study. These effects are quantified in a statistical manner by studying the mean of the $\mathrm{X}$ and $\mathrm{Y}$ distributions and the error on the mean in all cases of TS misalignment, source location and vacuum condition examined in the simulations.
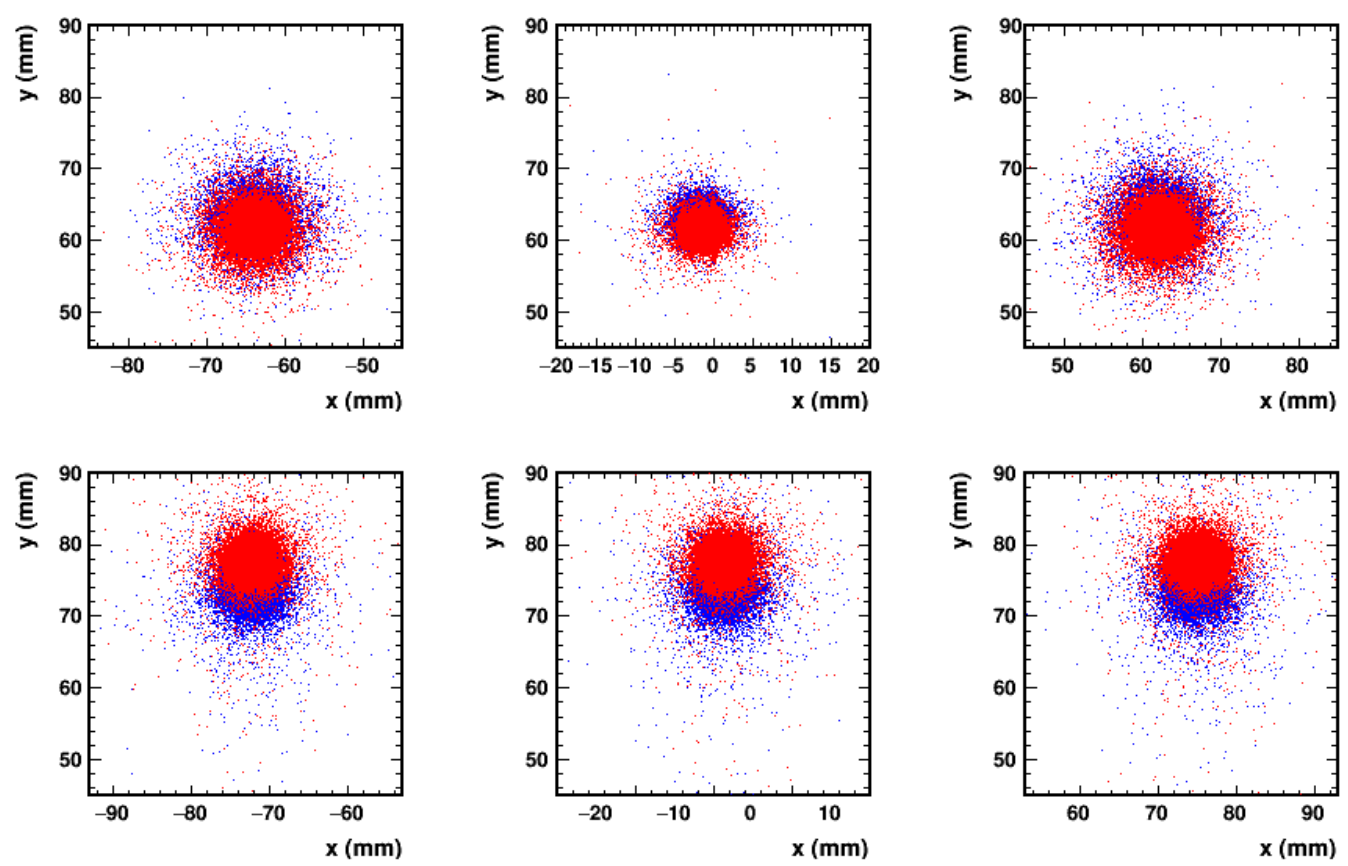

Figure 5-6: Electron beam profile in VD1 (top) and in VD8 (bottom) for 3 locations of the source in the XY plane near the production target: $-50 \mathrm{~mm}$ (left column), $0 \mathrm{~mm}$ (central column) and $+50 \mathrm{~mm}$ (right column) from the PS geometric axis. Scatter points (in red) simulated with a misaligned field (Mau10 $0.50^{\circ}$ rotation of TS about the $-Z$ axis) are drawn on top of scatter points (in blue) simulated with the design field (Mau10 default). 


\subsection{Results}

The results of the $\beta$ source simulation are shown using arrays of $3 \times 3$ plots: each array represents a virtual detector, while each plot represents a location of the source. The different maps are marked with different colors. The mean value and error on the mean are plotted. Error bars are not displayed when the number of particles in the beam spot is less than 50. In these cases, the error is considered unreliable on the grounds of Poisson statistics and thus only the central point is displayed, representing the mean value without its error. For brevity, the virtual detectors VD3, located in front of the TS3 collimator, and VD8, located after the TS5 collimator, are chosen to display the results. The choice is based on the facts that the results at VD3 always have significant statistics (prior to the cut placed by the TS3 collimator) and they are subject to significant effects of the field (after the TS2 solenoid bend) and of the medium (about $8 \mathrm{~m}$ path length in the air). Also, VD3 is close to the first suggested location of the real detector in the actual test, as discussed in subsection 5.1.1. VD8 is chosen because it is located in the end of the TS beam line, where the real detector would naturally be placed and where all effects of TS are included.

Table 5.2: Effect of the cut on outliers on Mau10 maps (default and rotated maps by $0.15^{\circ}$ and $0.50^{\circ}$ ) at VD3 for the X coordinate. Results without any cut and with the $4 \sigma$ cut are shown.

\begin{tabular}{|c|c|c|c|c|c|c|}
\hline \multirow[b]{2}{*}{$(\mathrm{X}[\mathrm{mm}], \mathrm{Y}[\mathrm{mm}])$} & \multicolumn{3}{|c|}{ Without $4 \sigma$ CUT } & \multicolumn{3}{|c|}{ WITH $4 \sigma$ сUT } \\
\hline & Default & $\begin{array}{l}\text { Rotation } 0.15^{\circ} \\
\text { about }-\mathrm{Z} \text { axis }\end{array}$ & $\begin{array}{l}\text { Rotation } 0.50^{\circ} \\
\text { about }-\mathrm{Z} \text { axis }\end{array}$ & Default & $\begin{array}{l}\text { Rotation } 0.15^{\circ} \\
\text { about }-\mathrm{Z} \text { axis }\end{array}$ & $\begin{array}{l}\text { Rotation } 0.50^{\circ} \\
\text { about }-\mathrm{Z} \text { axis }\end{array}$ \\
\hline$(3854,0)$ & $-73.729 \pm 0.017$ & $-73.821 \pm 0.049$ & $-73.401 \pm 0.502$ & $-73.733 \pm 0.014$ & $-73.834 \pm 0.043$ & $-73.401 \pm 0.502$ \\
\hline$(3904,0)$ & $-7.631 \pm 0.016$ & $-7.627 \pm 0.049$ & $-7.336 \pm 0.546$ & $-7.600 \pm 0.013$ & $-7.622 \pm 0.042$ & -7.225 \\
\hline$(3954,0)$ & $58.837 \pm 0.018$ & $58.814 \pm 0.044$ & $59.408 \pm 0.475$ & $58.832 \pm 0.014$ & $58.804 \pm 0.039$ & $59.408 \pm 0.475$ \\
\hline$(3854,50)$ & $-73.659 \pm 0.017$ & $-73.697 \pm 0.017$ & $-73.804 \pm 0.017$ & $-73.661 \pm 0.014$ & $-73.702 \pm 0.014$ & $-73.778 \pm 0.014$ \\
\hline$(3904,50)$ & $-7.548 \pm 0.017$ & $-7.562 \pm 0.016$ & $-7.615 \pm 0.016$ & $-7.521 \pm 0.013$ & $-7.551 \pm 0.013$ & $-7.632 \pm 0.013$ \\
\hline$(3954,50)$ & $58.925 \pm 0.018$ & $58.896 \pm 0.018$ & $58.818 \pm 0.017$ & $58.929 \pm 0.014$ & $58.896 \pm 0.014$ & $58.820 \pm 0.014$ \\
\hline$(3854,100)$ & $-70.835 \pm 0.151$ & $-72.806 \pm 0.024$ & $-73.642 \pm 0.016$ & $-70.910 \pm 0.138$ & $-72.849 \pm 0.019$ & $-73.663 \pm 0.013$ \\
\hline$(3904,100)$ & $-7.219 \pm 0.016$ & $-7.291 \pm 0.016$ & $-7.527 \pm 0.017$ & $-7.223 \pm 0.012$ & $-7.307 \pm 0.013$ & $-7.502 \pm 0.013$ \\
\hline$(3954,100)$ & $58.948 \pm 0.018$ & $59.056 \pm 0.017$ & $58.971 \pm 0.017$ & $59.004 \pm 0.013$ & $59.096 \pm 0.013$ & $58.983 \pm 0.013$ \\
\hline
\end{tabular}

\subsubsection{Study of the effect of outliers}

Electrons traveling along the transport line may undergo hard scattering off surrounding material. These scattered electrons deteriorate the uncertainty on the mean of the electron distribution at the virtual detectors, because they contribute to the mean uncertainty calculation quadratically. The effect of the outliers on the resolution of the test has been studied by applying cuts of the form:

$$
\left|x_{i}-\mu_{x}\right|<n \sigma_{x} \quad\left|y_{i}-\mu_{y}\right|<n \sigma_{y}
$$


Table 5.3: Effect of the cut on outliers on Mau10 maps (default and rotated maps by $0.15^{\circ}$ and $0.50^{\circ}$ ) at VD3 for the $Y$ coordinate. Results without any cut and with the $4 \sigma$ cut are shown.

\begin{tabular}{|c|c|c|c|c|c|c|}
\hline \multirow[b]{2}{*}{$(\mathrm{X}[\mathrm{mm}], \mathrm{Y}[\mathrm{mm}])$} & \multicolumn{3}{|c|}{ Without $4 \sigma$ cut } & \multicolumn{3}{|c|}{ With $4 \sigma$ сUт } \\
\hline & Default & $\begin{array}{l}\text { Rotation } 0.15^{\circ} \\
\text { about }-Z \text { axis }\end{array}$ & $\begin{array}{l}\text { Rotation } 0.50^{\circ} \\
\text { about }-\mathrm{Z} \text { axis }\end{array}$ & Default & $\begin{array}{l}\text { Rotation } 0.15^{\circ} \\
\text { about }-Z \text { axis }\end{array}$ & $\begin{array}{l}\text { Rotation } 0.50^{\circ} \\
\text { about }-\mathrm{Z} \text { axis }\end{array}$ \\
\hline$(3854,0)$ & $1.906 \pm 0.019$ & $8.272 \pm 0.122$ & $2.293 \pm 0.711$ & $1.928 \pm 0.015$ & $7.685 \pm 0.112$ & $2.420 \pm 0.700$ \\
\hline$(3904,0)$ & $1.990 \pm 0.017$ & $8.619 \pm 0.123$ & $0.926 \pm 0.760$ & $1.981 \pm 0.014$ & $7.984 \pm 0.112$ & $0.926 \pm 0.760$ \\
\hline$(3954,0)$ & $2.019 \pm 0.019$ & $8.580 \pm 0.116$ & $2.922 \pm 0.644$ & $2.028 \pm 0.015$ & $7.902 \pm 0.104$ & $2.922 \pm 0.644$ \\
\hline$(3854,50)$ & $68.014 \pm 0.019$ & $66.938 \pm 0.019$ & $64.544 \pm 0.019$ & $68.029 \pm 0.015$ & $66.973 \pm 0.015$ & $64.572 \pm 0.015$ \\
\hline$(3904,50)$ & $68.089 \pm 0.017$ & $67.116 \pm 0.017$ & $64.817 \pm 0.017$ & $68.091 \pm 0.014$ & $67.134 \pm 0.014$ & $64.830 \pm 0.014$ \\
\hline$(3954,50)$ & $68.171 \pm 0.019$ & $67.262 \pm 0.019$ & $65.083 \pm 0.018$ & $68.196 \pm 0.015$ & $67.263 \pm 0.015$ & $65.091 \pm 0.015$ \\
\hline$(3854,100)$ & $128.592 \pm 0.151$ & $131.876 \pm 0.026$ & $130.525 \pm 0.017$ & $128.624 \pm 0.133$ & $131.959 \pm 0.022$ & $130.613 \pm 0.015$ \\
\hline$(3904,100)$ & $134.225 \pm 0.017$ & $133.276 \pm 0.017$ & $130.989 \pm 0.017$ & $134.262 \pm 0.014$ & $133.292 \pm 0.014$ & $131.015 \pm 0.014$ \\
\hline$(3954,100)$ & $133.960 \pm 0.019$ & $133.297 \pm 0.017$ & $131.273 \pm 0.018$ & $134.067 \pm 0.016$ & $133.380 \pm 0.014$ & $131.320 \pm 0.015$ \\
\hline
\end{tabular}

A study of the cut at various $\sigma$ showed that the cut for $n=4$ gives stable results. Table 5.2 and Table 5.3 summarize the results of the effect of the cut on Mau10 maps in VD3 for the $\mathrm{X}$ and Y coordinate, respectively. Table 5.4 and Table 5.5 summarize the corresponding results in VD8. Comparing the left columns of the table with no cut applied with the right ones for each coordinate, it is clear that the $4 \sigma$ cut reduces the error. This means that outliers from hard scattering affect significantly the resolution of the test and we need to remove them with the $4 \sigma$ cut.

\subsubsection{Study of the effect of detector resolution and secondary particles}

In order to quantify the effects of traversed material at the default vacuum of the study (1 Torr), we compare the results with and without $\delta$ rays and detector resolution $(300 \mu \mathrm{m})$. These medium effects (detector resolution and $\delta$ rays) play a minor role in most cases: Figure 5-7 and Figure 5-8 show results for Mau10 default maps at VD3 and VD8, respectively. The mean values and the errors of red points (no detector resolution and $\delta$ rays) and blue points (detector resolution and $\delta$ rays included) are comparable. Table 5.6 and Table 5.7 summarize the results for Mau10 default maps at VD3 and VD8, respectively. 
Table 5.4: Effect of the cut on outliers on Mau10 maps (default and rotated maps by $0.15^{\circ}$ and $0.50^{\circ}$ ) at VD8 for the X coordinate. Results without any cut and with the $4 \sigma$ cut are shown.

\begin{tabular}{|c|c|c|c|c|c|c|}
\hline \multirow[b]{2}{*}{$(\mathrm{X}[\mathrm{mm}], \mathrm{Y}[\mathrm{mm}])$} & \multicolumn{3}{|c|}{ Without $4 \sigma$ CUT } & \multicolumn{3}{|c|}{ WITH $4 \sigma$ CUT } \\
\hline & Default & $\begin{array}{l}\text { Rotation } 0.15^{\circ} \\
\text { about }-\mathrm{Z} \text { axis }\end{array}$ & $\begin{array}{l}\text { Rotation } 0.50^{\circ} \\
\text { about }-\mathrm{Z} \text { axis }\end{array}$ & Default & $\begin{array}{l}\text { Rotation } 0.15^{\circ} \\
\text { about }-\mathrm{Z} \text { axis }\end{array}$ & $\begin{array}{l}\text { Rotation } 0.50^{\circ} \\
\text { about }-\mathrm{Z} \text { axis }\end{array}$ \\
\hline$(3854,0)$ & $-72.218 \pm 0.019$ & $-72.271 \pm 0.047$ & $0 \pm 0$ & $-72.215 \pm 0.016$ & $-72.265 \pm 0.040$ & $0 \pm 0$ \\
\hline$(3904,0)$ & $1.387 \pm 0.022$ & $1.361 \pm 0.063$ & $1.161 \pm 2.545$ & $1.370 \pm 0.017$ & $1.420 \pm 0.042$ & $1.161 \pm 2.545$ \\
\hline$(3954,0)$ & $74.942 \pm 0.020$ & $74.951 \pm 0.041$ & $69.848 \pm 0.000$ & $74.952 \pm 0.016$ & $74.967 \pm 0.037$ & $0 \pm 0$ \\
\hline$(3854,5)$ & $-72.251 \pm 0.019$ & $-72.189 \pm 0.019$ & $-72.168 \pm 0.018$ & $-72.250 \pm 0.016$ & $-72.211 \pm 0.016$ & $-72.175 \pm 0.016$ \\
\hline$(3904,5)$ & $1.364 \pm 0.020$ & $1.354 \pm 0.019$ & $1.405 \pm 0.023$ & $1.370 \pm 0.016$ & $1.352 \pm 0.016$ & $1.417 \pm 0.016$ \\
\hline$(3954,5)$ & $74.942 \pm 0.018$ & $74.940 \pm 0.018$ & $74.979 \pm 0.019$ & $74.953 \pm 0.016$ & $74.950 \pm 0.016$ & $75.009 \pm 0.016$ \\
\hline$(3854,100)$ & $0 \pm 0$ & $-73.611 \pm 1.905$ & $-77.991 \pm 2.523$ & $0 \pm 0$ & $-73.611 \pm 1.905$ & $-77.991 \pm 2.523$ \\
\hline$(3904,100)$ & $-6.951 \pm 4.125$ & $-5.200 \pm 3.693$ & $6.085 \pm 4.913$ & $-3.539 \pm 2.419$ & $-2.523 \pm 2.651$ & $1.132 \pm 0.958$ \\
\hline$(3954,100)$ & $30.597 \pm 19.808$ & $-56.826 \pm 66.057$ & $67.237 \pm 0.000$ & $30.597 \pm 19.808$ & $-56.826 \pm 66.057$ & $0 \pm 0$ \\
\hline
\end{tabular}

Table 5.5: Effect of the cut on outliers on Mau10 maps (default and rotated maps by $0.15^{\circ}$ and $0.50^{\circ}$ ) at VD8 for the $Y$ coordinate. Results without any cut and with the $4 \sigma$ cut are shown.

\begin{tabular}{c||ccc|ccc}
\hline \hline & \multicolumn{3}{c|}{ WITHOUT $4 \sigma$ CUT } & \multicolumn{3}{c}{ WITH $4 \sigma$ CUT } \\
\hline$(X$ [mm], Y [mm] $)$ & Default & $\begin{array}{c}\text { Rotation } \mathbf{0 . 1 5} \\
\text { about }-\mathbf{Z} \text { axis }\end{array}$ & $\begin{array}{c}\text { Rotation } \mathbf{0 . 5 0} \\
\text { about }-\mathbf{Z} \text { axis }\end{array}$ & Default & $\begin{array}{c}\text { Rotation } \mathbf{0 . 1 5} \\
\text { about }-\mathbf{Z} \text { axis }\end{array}$ & $\begin{array}{c}\text { Rotation } \mathbf{0 . 5 0}^{\circ} \\
\text { about }-\mathbf{Z} \text { axis }\end{array}$ \\
\hline$(3854,0)$ & $-0.158 \pm 0.020$ & $13.437 \pm 0.234$ & $0.000 \pm 0.000$ & $-0.136 \pm 0.017$ & $13.374 \pm 0.232$ & $0.000 \pm 0.000$ \\
$(3904,0)$ & $-0.029 \pm 0.023$ & $12.599 \pm 0.222$ & $22.564 \pm 2.344$ & $-0.006 \pm 0.017$ & $12.517 \pm 0.218$ & $22.564 \pm 2.344$ \\
$(3954,0)$ & $0.049 \pm 0.020$ & $12.440 \pm 0.211$ & $13.850 \pm 0.000$ & $0.059 \pm 0.017$ & $12.207 \pm 0.206$ & $0.000 \pm 0.000$ \\
$(3854,50)$ & $73.533 \pm 0.021$ & $74.729 \pm 0.022$ & $77.660 \pm 0.022$ & $73.597 \pm 0.017$ & $74.804 \pm 0.017$ & $77.735 \pm 0.017$ \\
$(3904,50)$ & $73.604 \pm 0.023$ & $74.882 \pm 0.022$ & $77.685 \pm 0.028$ & $73.689 \pm 0.017$ & $74.933 \pm 0.017$ & $77.775 \pm 0.018$ \\
$(3954,50)$ & $73.683 \pm 0.022$ & $74.939 \pm 0.021$ & $77.799 \pm 0.021$ & $73.747 \pm 0.017$ & $75.013 \pm 0.017$ & $77.859 \pm 0.018$ \\
$(3854,100)$ & $0 \pm 0$ & $98.549 \pm 1.942$ & $89.912 \pm 4.574$ & $0 \pm 0$ & $98.549 \pm 1.942$ & $89.912 \pm 4.574$ \\
$(3904,100)$ & $101.097 \pm 6.183$ & $101.096 \pm 6.930$ & $109.007 \pm 3.993$ & $104.875 \pm 5.026$ & $101.096 \pm 6.930$ & $109.007 \pm 3.993$ \\
$(3954,100)$ & $12.872 \pm 38.444$ & $-21.058 \pm 50.550$ & $93.098 \pm 0.000$ & $12.872 \pm 38.444$ & $-21.058 \pm 50.550$ & $0.000 \pm 0.000$ \\
\hline \hline
\end{tabular}


Table 5.6: Effect of the detector resolution and $\delta$ rays on Mau10 default maps at VD3 for the $X$ and $Y$ coordinates.

\begin{tabular}{c||cc|cc}
\hline \hline & \multicolumn{2}{c||}{ X } & \multicolumn{2}{c}{ Y } \\
\hline Source Location & \multicolumn{2}{c}{ Detector Resolution AND Secondary Particles } \\
\hline \multicolumn{1}{c||}{ X [mm], Y [mm] $)$} & Without & With & Without & With \\
\hline$(3854,0)$ & $-73.729 \pm 0.017$ & $-73.723 \pm 0.017$ & $1.906 \pm 0.019$ & $1.922 \pm 0.018$ \\
$(3904,0)$ & $-7.631 \pm 0.016$ & $-7.622 \pm 0.015$ & $1.990 \pm 0.017$ & $1.982 \pm 0.017$ \\
$(3954,0)$ & $58.837 \pm 0.018$ & $58.845 \pm 0.017$ & $2.019 \pm 0.019$ & $2.013 \pm 0.018$ \\
$(3854,50)$ & $-73.659 \pm 0.017$ & $-73.657 \pm 0.017$ & $68.014 \pm 0.019$ & $68.000 \pm 0.018$ \\
$(3904,50)$ & $-7.548 \pm 0.0166$ & $-7.538 \pm 0.015$ & $68.089 \pm 0.017$ & $68.092 \pm 0.016$ \\
$(3954,50)$ & $58.925 \pm 0.0185$ & $58.934 \pm 0.016$ & $68.171 \pm 0.019$ & $68.184 \pm 0.018$ \\
$(3854,100)$ & $-70.835 \pm 0.151$ & $-70.851 \pm 0.149$ & $128.592 \pm 0.151$ & $128.596 \pm 0.147$ \\
$(3904,100)$ & $-7.219 \pm 0.016$ & $-7.220 \pm 0.015$ & $134.225 \pm 0.017$ & $134.233 \pm 0.016$ \\
$(3954,100)$ & $58.948 \pm 0.018$ & $58.952 \pm 0.016$ & $133.960 \pm 0.019$ & $133.976 \pm 0.018$ \\
\hline \hline
\end{tabular}

Table 5.7: Effect of the detector resolution and $\delta$ rays on Mau10 default maps at VD8 for the X and $Y$ coordinates. At $(X, Y) \equiv(3854 \mathrm{~mm}, 100 \mathrm{~mm})$ no electrons make it through VD8 in this case.

\begin{tabular}{|c|c|c|c|c|}
\hline & \multicolumn{2}{|c|}{$x$} & \multicolumn{2}{|c|}{$\mathrm{Y}$} \\
\hline SOURCE LOCATION & \multicolumn{4}{|c|}{ Detector Resolution and Secondary Particles } \\
\hline$(\mathrm{X}[\mathrm{mm}], \mathrm{Y}[\mathrm{mm}])$ & Without & WiтH & Without & With \\
\hline$(3854,0)$ & $-72.218 \pm 0.019$ & $-72.222 \pm 0.017$ & $-0.158 \pm 0.020$ & $-0.174 \pm 0.019$ \\
\hline$(3904,0)$ & $1.387 \pm 0.022$ & $1.363 \pm 0.0176$ & $-0.029 \pm 0.023$ & $-0.026 \pm 0.018$ \\
\hline$(3954,0)$ & $74.942 \pm 0.020$ & $74.964 \pm 0.018$ & $0.049 \pm 0.020$ & $0.038 \pm 0.018$ \\
\hline$(3854,50)$ & $-72.251 \pm 0.019$ & $-72.247 \pm 0.017$ & $73.533 \pm 0.021$ & $73.517 \pm 0.019$ \\
\hline$(3904,50)$ & $1.364 \pm 0.020$ & $1.365 \pm 0.017$ & $73.603 \pm 0.023$ & $73.601 \pm 0.021$ \\
\hline$(3954,50)$ & $74.942 \pm 0.018$ & $74.941 \pm 0.017$ & $73.683 \pm 0.022$ & $73.656 \pm 0.020$ \\
\hline$(3854,100)$ & $0 \pm 0$ & $0 \pm 0$ & $0 \pm 0$ & $0 \pm 0$ \\
\hline$(3904,100)$ & $-6.951 \pm 4.125$ & $0.020 \pm 0.615$ & $101.097 \pm 6.183$ & $113.549 \pm 1.986$ \\
\hline$(3954,100)$ & $30.597 \pm 19.808$ & $65.378 \pm 0.136$ & $12.872 \pm 38.444$ & $98.689 \pm 2.169$ \\
\hline
\end{tabular}



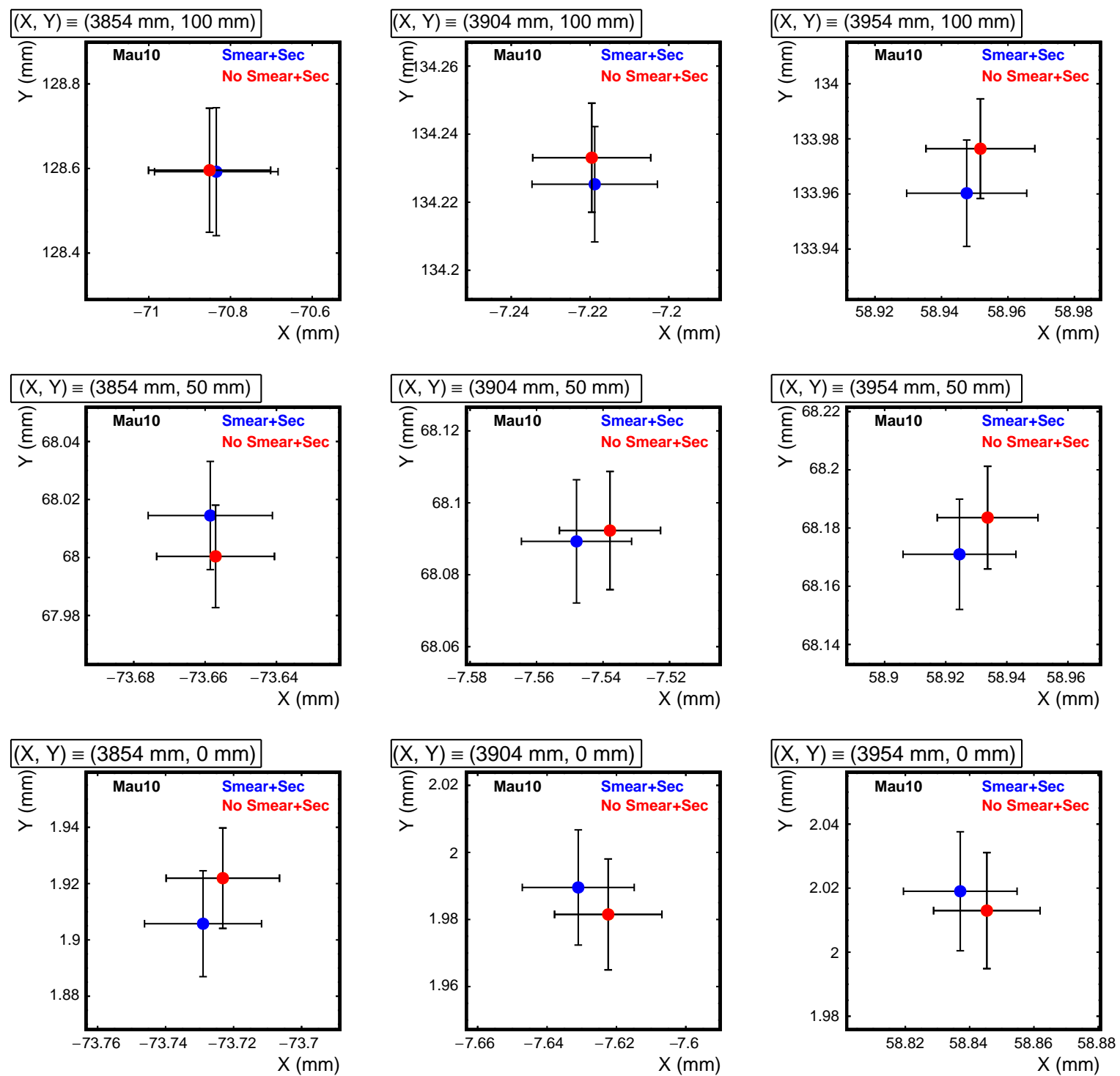

Figure 5-7: Study of the effect of detector resolution and $\delta$ rays using Mau10 default maps. No cut on outliers is applied. Simulation results which include detector resolution of $300 \mu \mathrm{m}$ and $\delta$ rays are represented by red points, blue points stand for results without detector resolution and $\delta$ rays. The results are shown at VD3. 

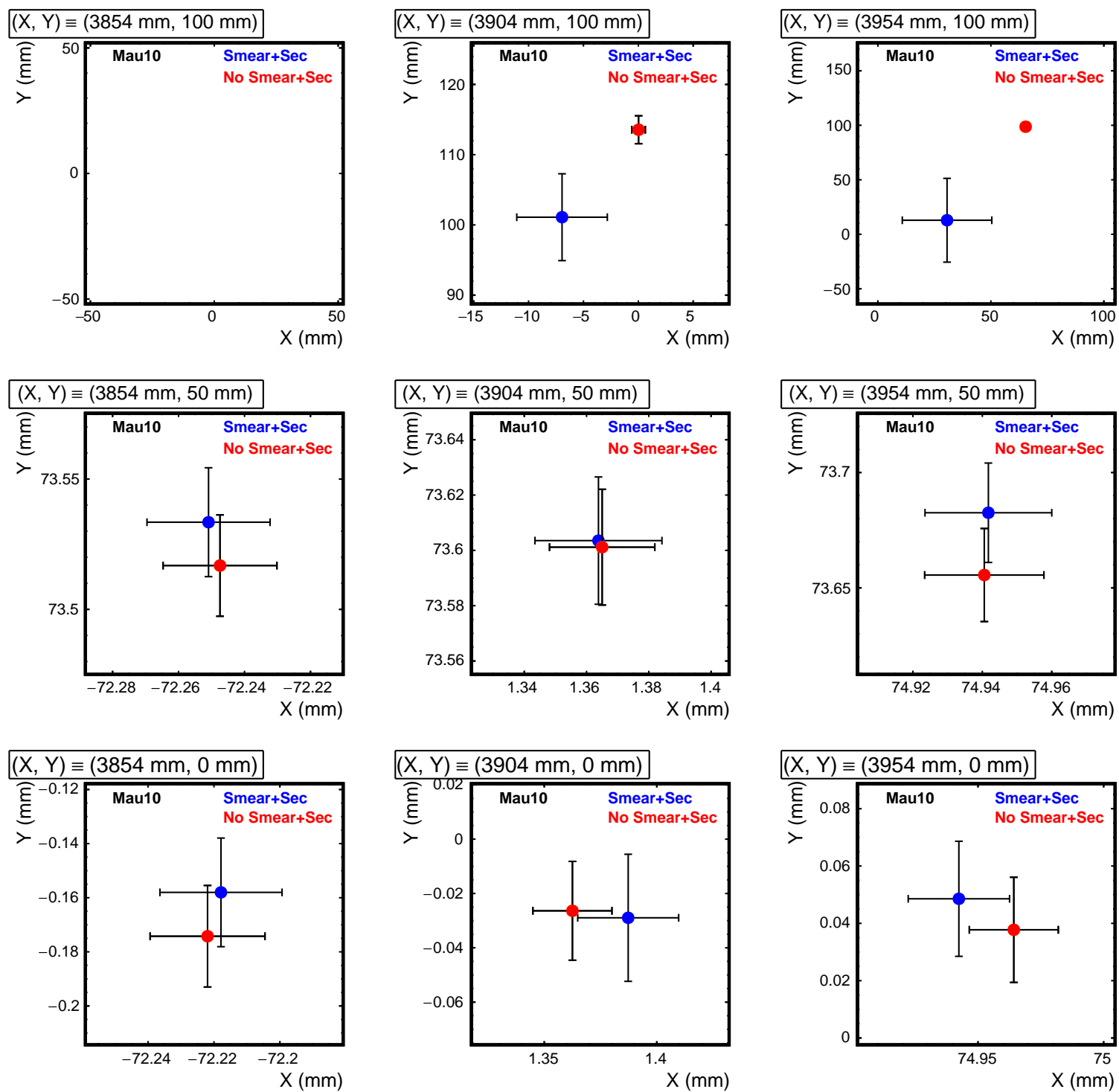

Figure 5-8: Study of the effect of detector resolution and $\delta$ rays using Mau10 default maps. No cut on outliers is applied. Simulation results which include detector resolution of $300 \mu \mathrm{m}$ and $\delta$ rays are represented by red points, blue points stand for results without detector resolution and $\delta$ rays. The results are shown at VD8. The upper left window $((X, Y) \equiv(3854 \mathrm{~mm}, 100 \mathrm{~mm}))$ shows no points because no electrons make it through VD8 in this case. 


\subsubsection{Rotations}

Simulations using SolCalc maps for $1^{\circ}$ and $0.1^{\circ}$ rotation of TS with respect to the $\mathrm{X}$ axis and Mau10 rotation of $0.50^{\circ}$ and $0.15^{\circ}$ with respect to to the $-\mathrm{Z}$ axis are compared with the nominal results (SolCalc default maps and Mau10 default maps, respectively). Air pressure of 1 Torr is assumed in all simulations.

Figure 5-9 shows the $X Y$ distribution of the electrons, including primary electrons from the source and $\delta$ rays, in local coordinates for Mau10 default maps. The detector resolution is included and no cut on outliers is applied. The spot of electrons moves in the various virtual detectors. The beam size of a few $\mathrm{mm}$ is evident.

Figure 5-10 shows the momentum spectrum of electrons coming from the ${ }^{90} \mathrm{Sr} /{ }^{90} \mathrm{Y}$ source in VD1 (5-10(a)), VD3 (5-10(b)) and VD8 (5-10(c)). The results include the $4 \sigma$ cut for the outliers, $\delta$ rays (but no $\gamma$ rays) and detector resolution of $300 \mu \mathrm{m}$ in X and Y. The maps used are the default Mau10 maps and the $\beta$ source is on the axis. The spectrum is the sum of the ${ }^{90} \mathrm{Sr}$ and ${ }^{90} \mathrm{Y}$ spectra: in $5-10$ (a) the first peak corresponds to ${ }^{90} \mathrm{Sr}$ and the second one to ${ }^{90} \mathrm{Y}$. The spectrum remains the same as long as the particles are in TSu (i.e. VD1, VD2, VD3, VD4), but after passing the TS3 collimator the low momentum peak is suppressed (5-10(c)), because many low momentum particles have been stopped by the collimator.

Figure 5-11 and Figure 5-12 show the results for Mau10 maps at VD3 and VD8, respectively: the default field (black points) is compared with the rotated ones (blue points for rotation of $0.50^{\circ}$ and red points for rotation of $0.15^{\circ}$, both about the $-\mathrm{Z}$ axis). The results include the $4 \sigma$ cut for the outliers, $\delta$ rays (but no $\gamma$ rays) and detector resolution of $300 \mu \mathrm{m}$ in X and Y. Each point is located at the mean value of $X$ and $Y$ of the electron beam detected at the virtual detectors, and the error bars represent the errors on the mean values. The figures show results for the $\beta$ source moved above the $X Z$ plane in steps of $50 \mathrm{~mm}$. In Figure 5-13 and Figure 5-14 the source is moved above the $X Z$ plane in steps of $5 \mathrm{~mm}$. Figure 5-15 and Figure 5-16 show results for the $\beta$ source moved below the $\mathrm{XZ}$ plane in steps of $5 \mathrm{~mm}$.

Figure 5-17 and Figure 5-18 show the results for SolCalc maps rotated by $1^{\circ}$ at the virtual detectors VD3 and VD8, respectively. Figure 5-19 and Figure 5-20 show the results for $0.1^{\circ}$ rotation of TS about the $X$ axis at the virtual detectors VD3 and VD8, respectively. For each plot, 5 points are displayed: the black point corresponds to the default maps, the colored ones correspond to the rotated field maps with different TSu and TSd rotations. For brevity, the figures show results only for the $\beta$ source moved above the $\mathrm{XZ}$ plane in steps of $50 \mathrm{~mm}$, since these source locations capture the bulk effects of the rotations.

These figures show that the $\beta$ source test is not only sensitive to exaggerated TS rotations of $1^{\circ}$ with respect to $X$ axis and $0.50^{\circ}$ with respect to $-Z$ axis, but also to smaller rotations of $0.15^{\circ}$ 

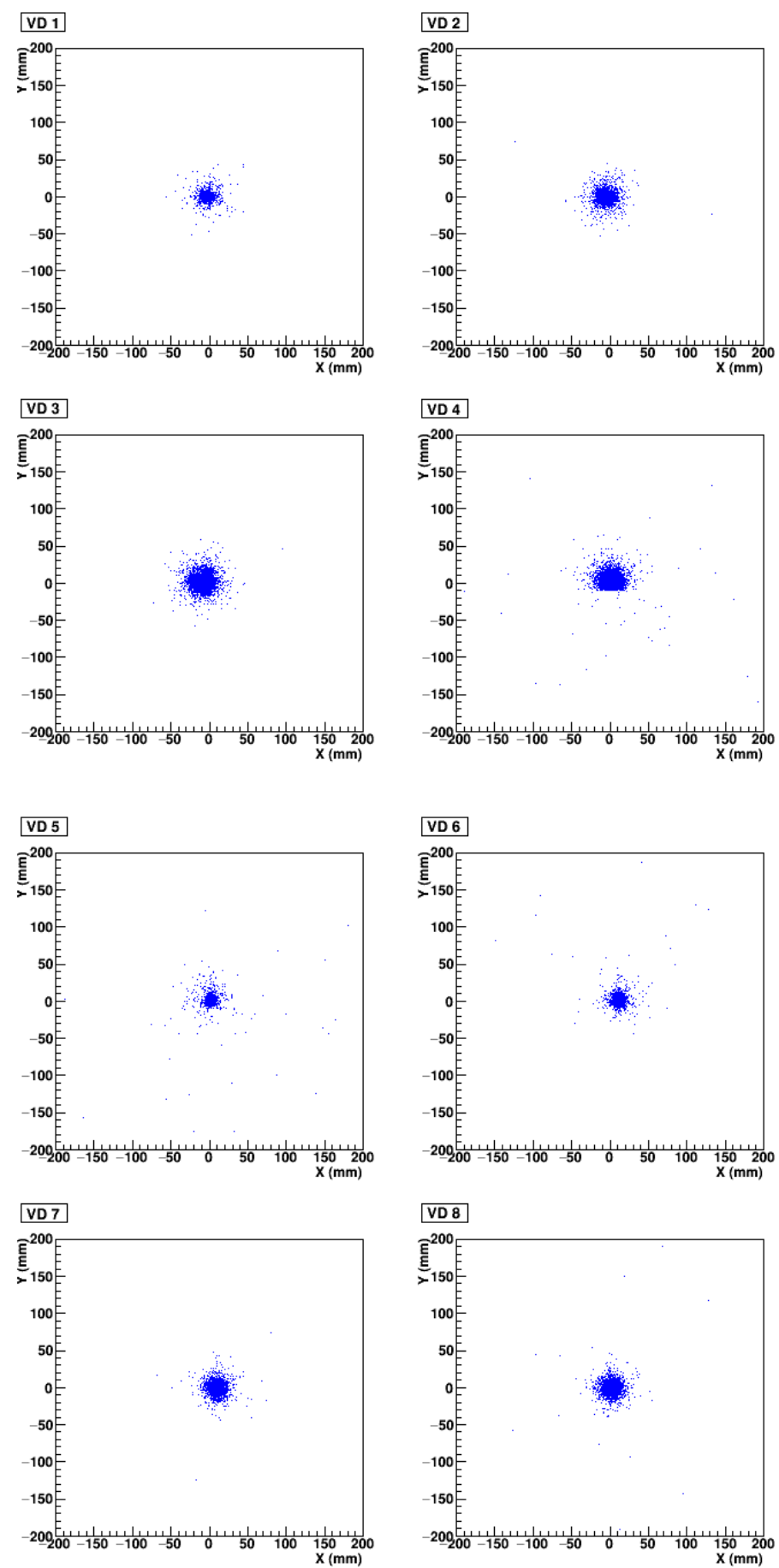

Figure 5-9: $X Y$ distribution of electrons at the 8 virtual detectors. The default Mau10 maps are used, the source is on axis, spatial detector resolution and $\delta$ rays ( $\gamma$ and $\delta$ rays) are included. The detector resolution is included and no cut on outliers is applied.

with respect to $-\mathrm{Z}$ axis and of $0.1^{\circ}$ with respect to to $\mathrm{X}$ axis. As expected, the largest position variations in the beam spots are in the $\mathrm{Y}$ axis, since the rotations are performed in the $\mathrm{YZ}$ and $\mathrm{XY}$ 

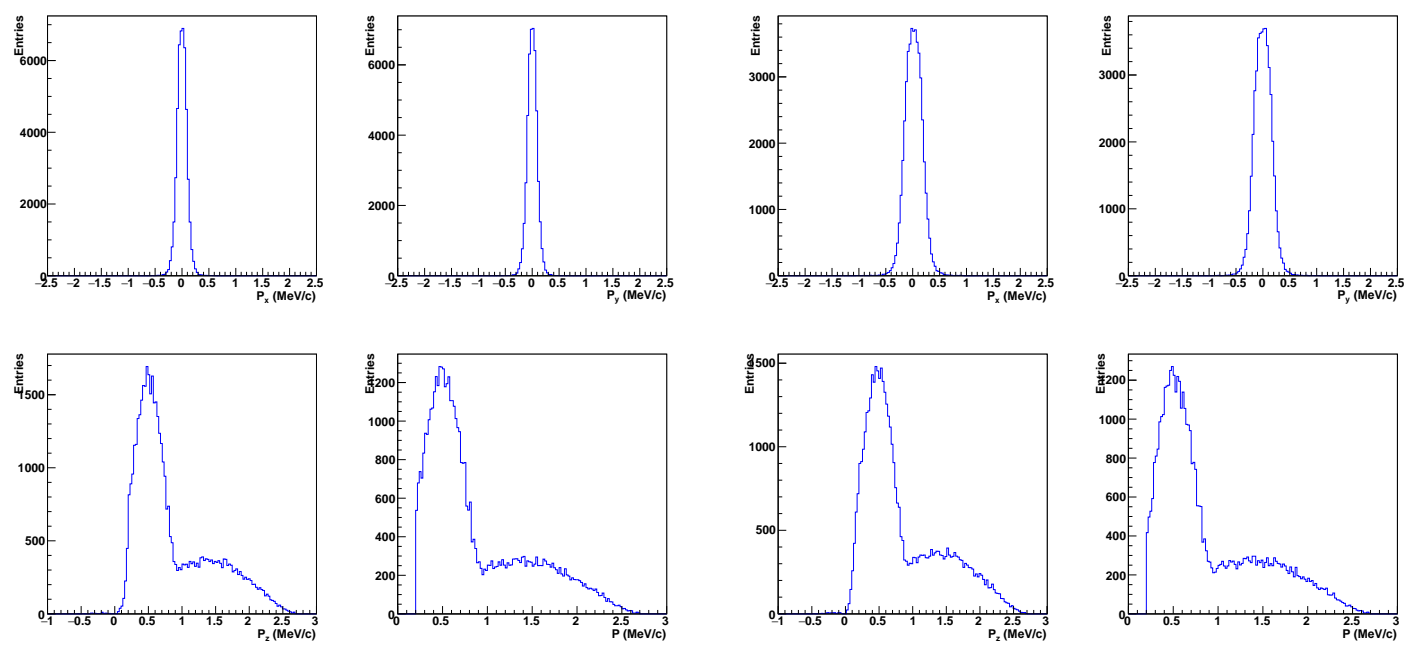

(a) VD1

(b) VD3
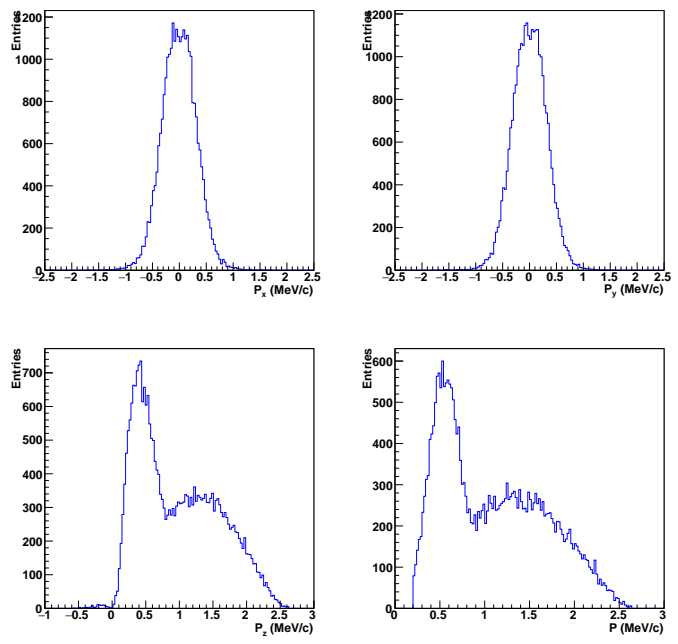

(c) VD8

Figure 5-10: Momentum spectra of electrons in VD1 (5-10(a)), VD3 (5-10(b)) and VD8 (5-10(c)). The nominal Mau10 maps are used, the source is on axis, spatial detector resolution and $4 \sigma$ cut are applied, $\delta$ rays (no $\gamma$ rays) are included. For each virtual detector, the three components $p_{x}$ (upper left plot), $p_{y}$ (upper right plot), $p_{z}$ (lower left plot) and the magnitude $p$ (lower right plot) are shown in each of the 4-window plots 5-10(a), 5-10(b), 5-10(c).

planes. With the assumed medium effects and detector resolution, the $\beta$ source test can resolve TS misalignments down to $5 \mathrm{~mm}\left(0.1^{\circ}\right.$ rotation). However, it should be stressed that the test alone can not resolve the type of misalignment, because different misalignments may have the same effect on the low momentum electron beam from the same source location. The capacity of the test relies on detecting misalignment effects in general and, if these effects are found significant, a new survey of the solenoid alignment with mechanical means is dictated. 

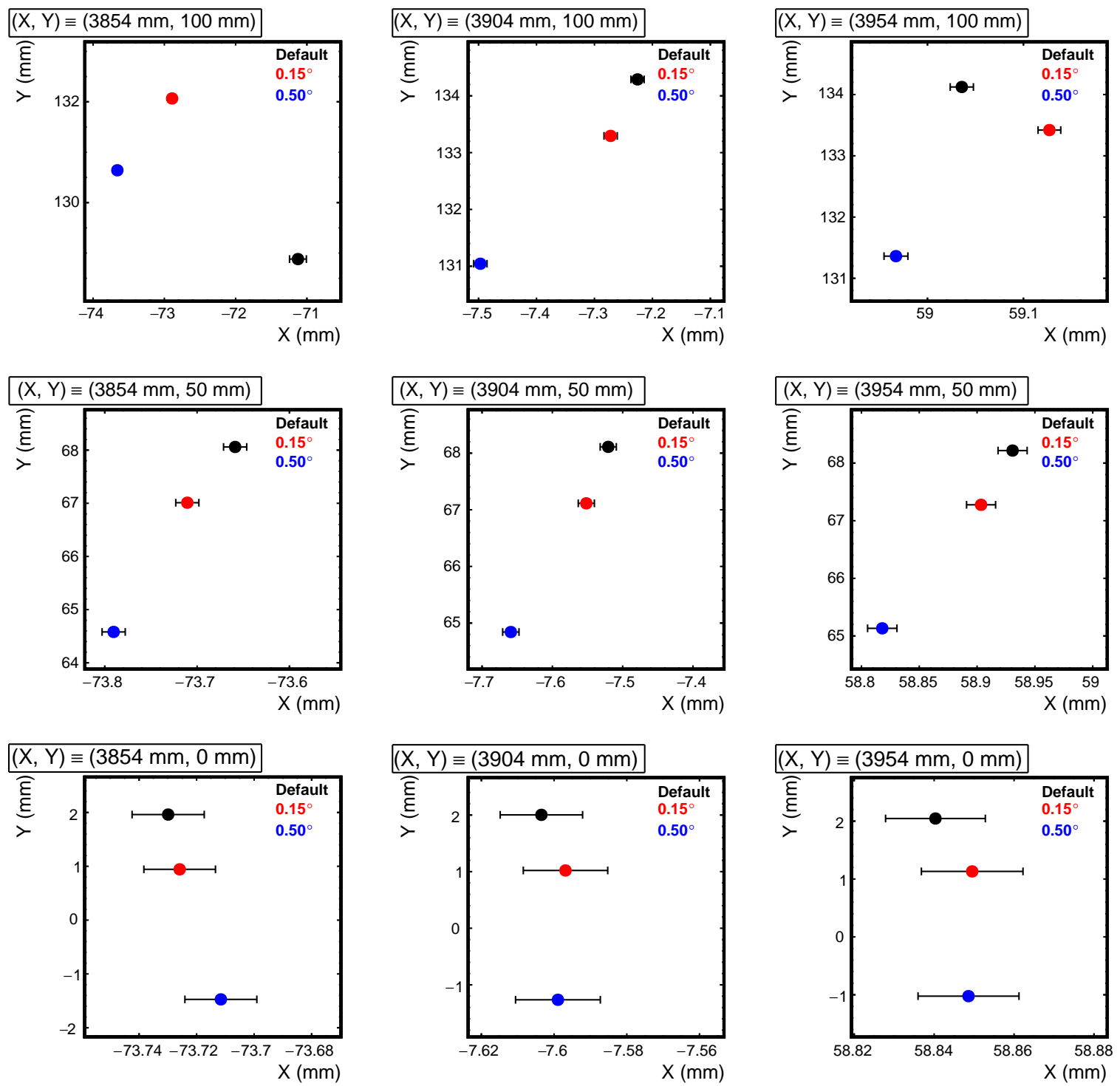

Figure 5-11: Results for Mau10 rotated maps with respect to to $-Z$ axis at VD3. The source is located above the XZ plane and it is moved in steps of $50 \mathrm{~mm}$. Default Mau10 maps are represented with black points, TS rotations about the $-Z$ axis by $0.15^{\circ}$ are in red and by $0.50^{\circ}$ are in blue. Errors on the $Y$ axis are not visible because of different scales in the $X$ and $Y$ axis. 

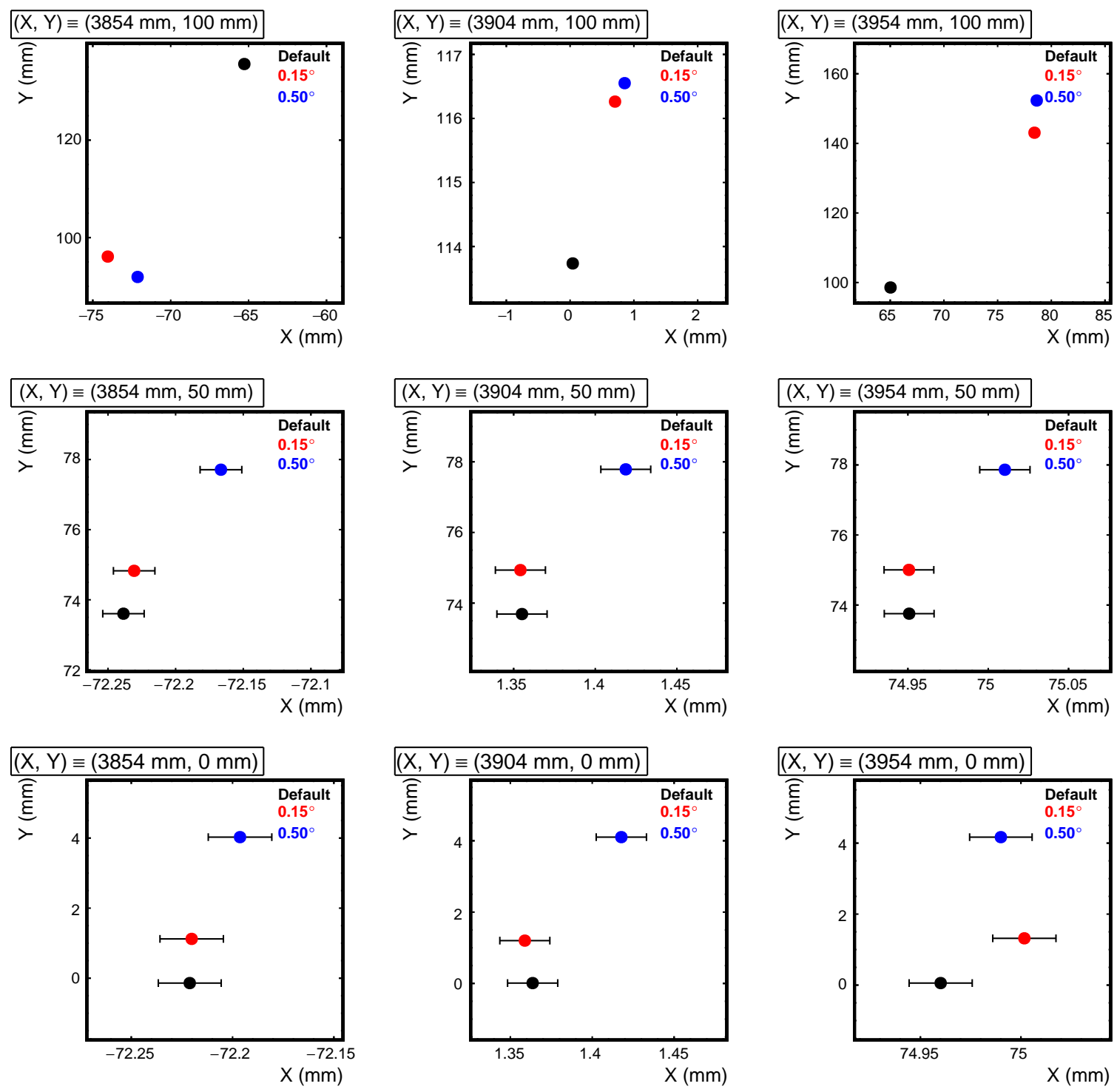

Figure 5-12: Results for Mau10 rotated maps with respect to to $-Z$ axis at VD8. The source is located above the XZ plane and it is moved in steps of $50 \mathrm{~mm}$. Default Mau10 maps are represented with black points, TS rotations about the $-Z$ axis by $0.15^{\circ}$ are in red and by $0.50^{\circ}$ are in blue. Errors on the $Y$ axis are not visible because of different scales in the $X$ and $Y$ axis. 

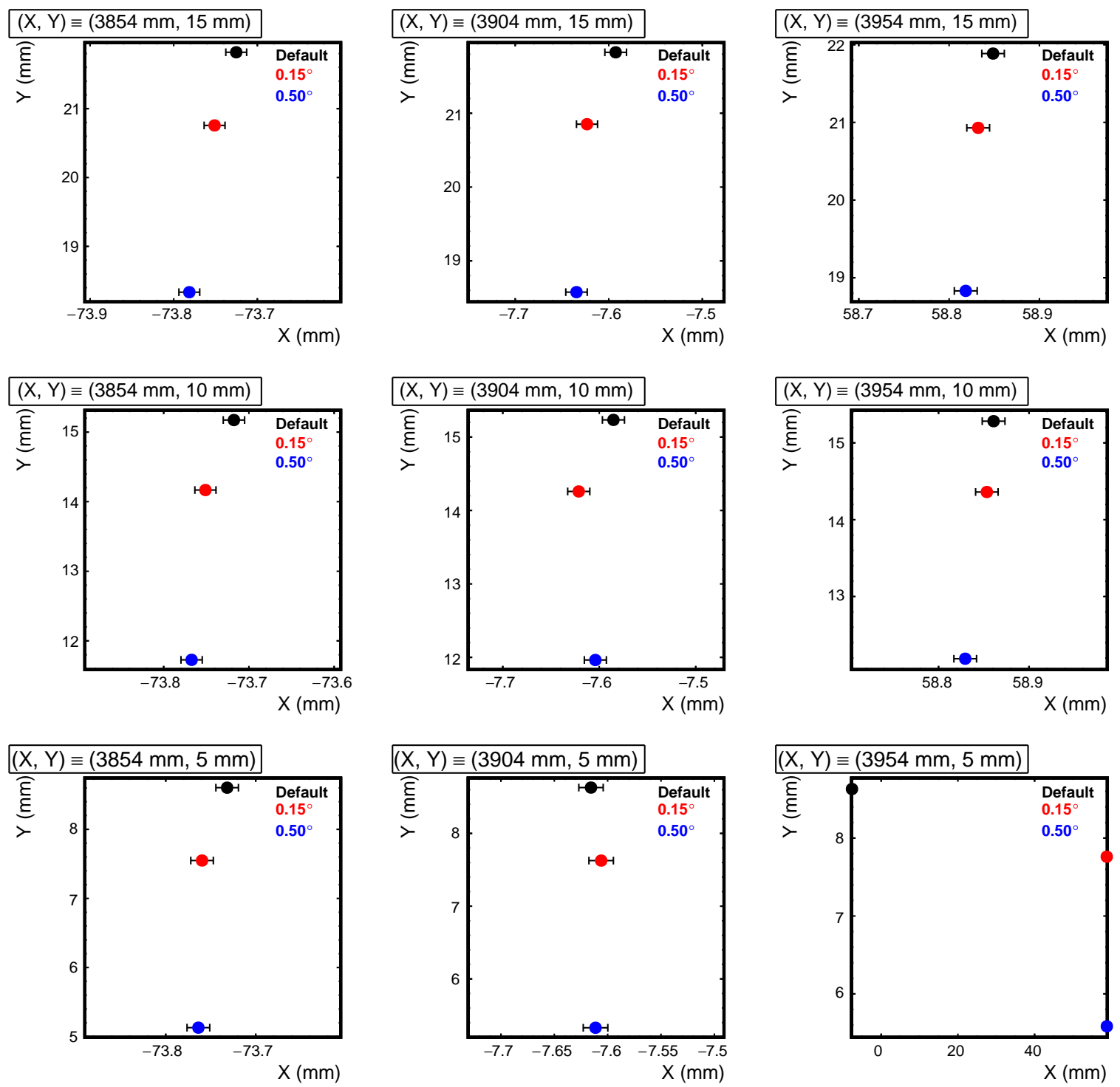

Figure 5-13: Results for Mau10 rotated maps with respect to to $-Z$ axis at VD3. The $\beta$ source is located above the $X Z$ plane and it is moved in steps of $5 \mathrm{~mm}$. Default Mau10 maps are represented with black points, TS rotations about the $-Z$ axis by $0.15^{\circ}$ are in red and by $0.50^{\circ}$ are in blue. Errors on the $Y$ axis are not visible because of different scales in the $X$ and $Y$ axis. 

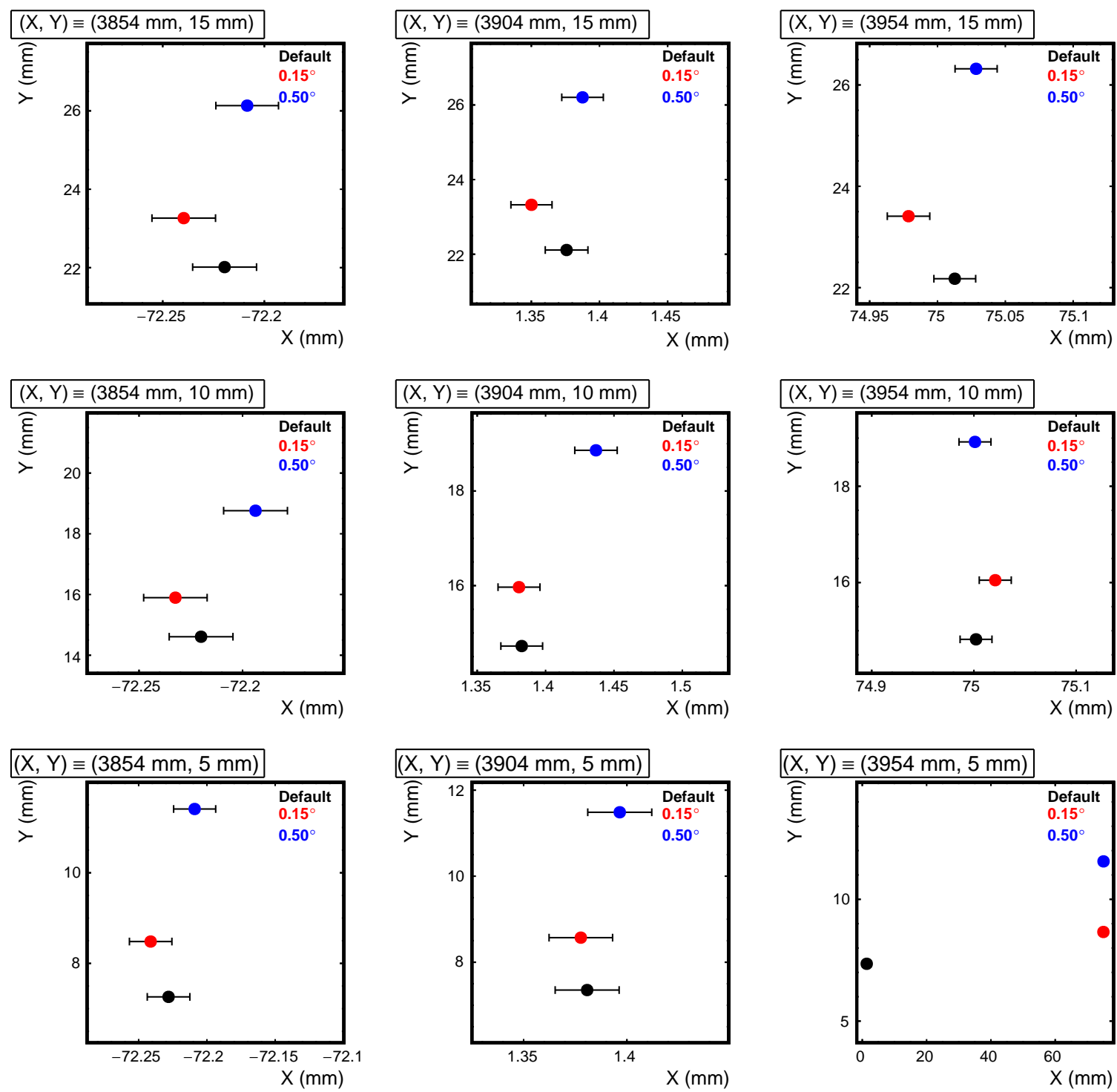

Figure 5-14: Results for Mau10 rotated maps with respect to to $-Z$ axis at VD8. The $\beta$ source is located above the $X Z$ plane and it is moved in steps of $5 \mathrm{~mm}$. Default Mau10 maps are represented with black points, TS rotations about the $-Z$ axis by $0.15^{\circ}$ are in red and by $0.50^{\circ}$ are in blue. Errors on the $Y$ axis are not visible because of different scales in the $X$ and $Y$ axis. 

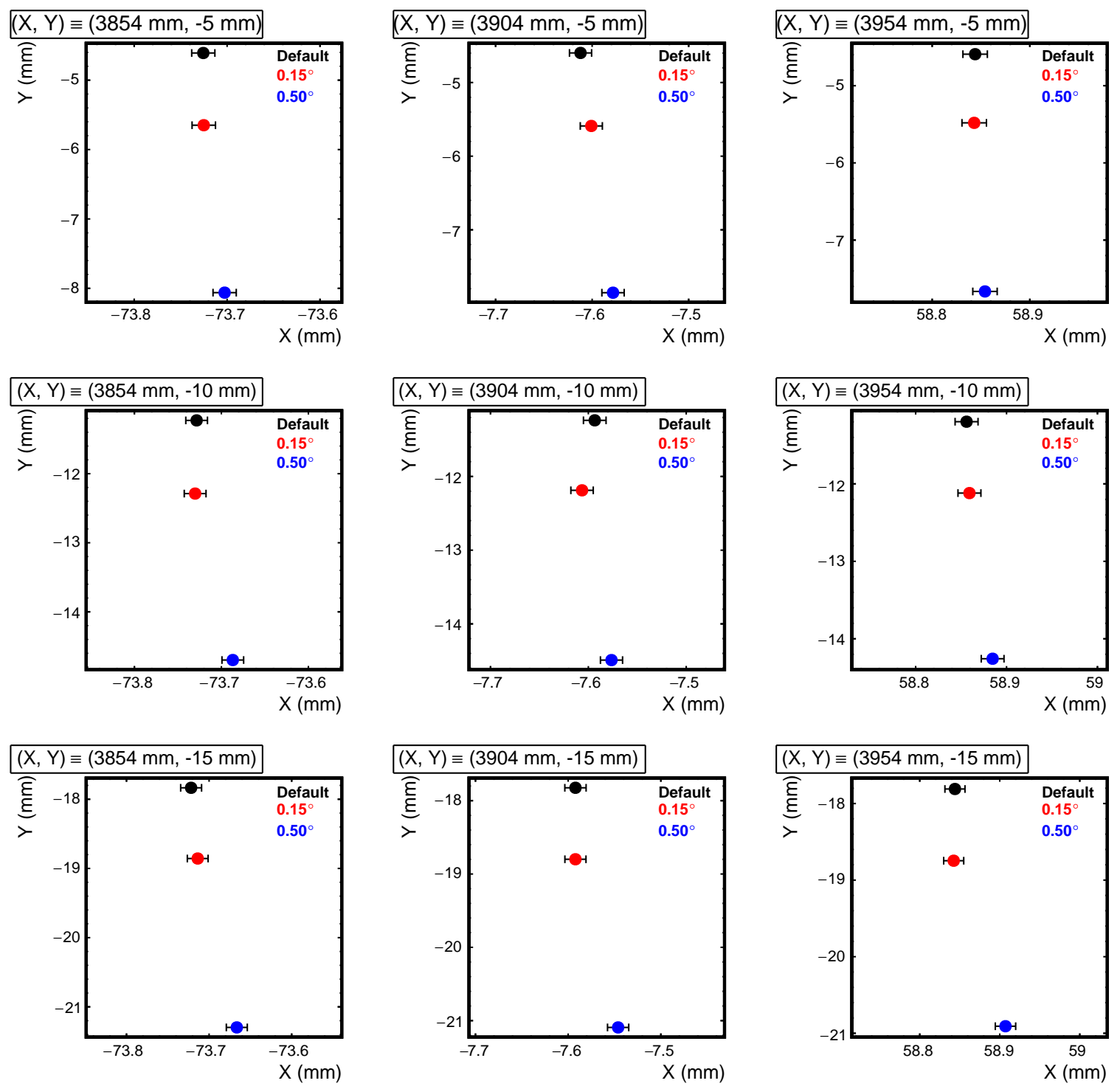

Figure 5-15: Results for Mau10 rotated maps with respect to to $-Z$ axis at VD3. The $\beta$ source is located below the $X Z$ plane and it is moved in steps of $5 \mathrm{~mm}$. Default Mau10 maps are represented with black points, TS rotations about the $-Z$ axis by $0.15^{\circ}$ are in red and by $0.50^{\circ}$ are in blue. Errors on the $Y$ axis are not visible because of different scales in the $X$ and $Y$ axis. 

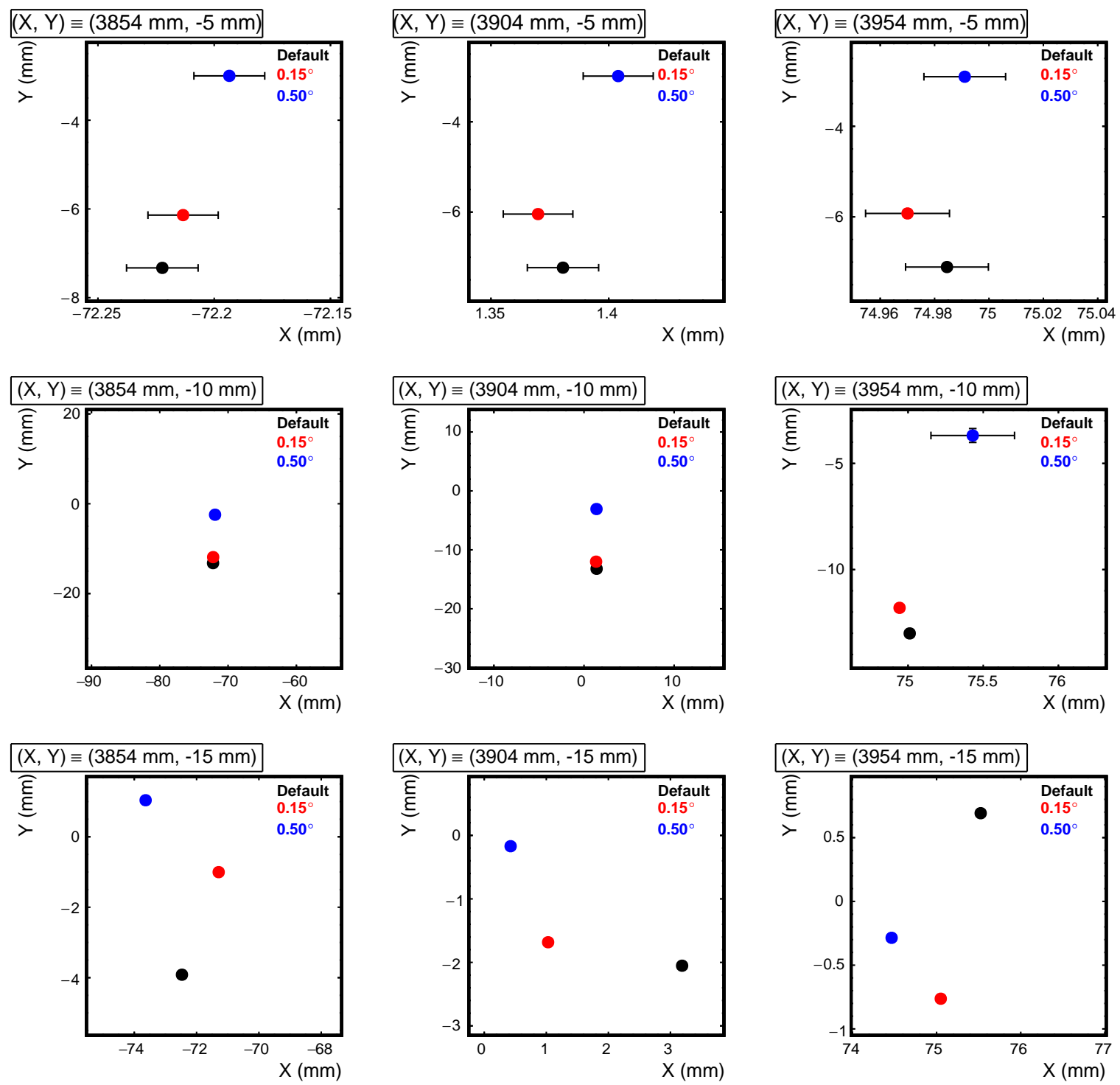

Figure 5-16: Results for Mau10 rotated maps with respect to to $-Z$ axis at VD8. The $\beta$ source is located below the $X Z$ plane and it is moved in steps of $5 \mathrm{~mm}$. Default Mau10 maps are represented with black points, TS rotations about the $-\mathrm{Z}$ axis by $0.15^{\circ}$ are in red and by $0.50^{\circ}$ are in blue. The points in the middle and bottom plots are missing error bars because of the condition of the error being set to zero when the number of particles detected is less than 50, as described in the text. Errors on the Y axis are not visible because of different scales in the $X$ and $Y$ axis. 

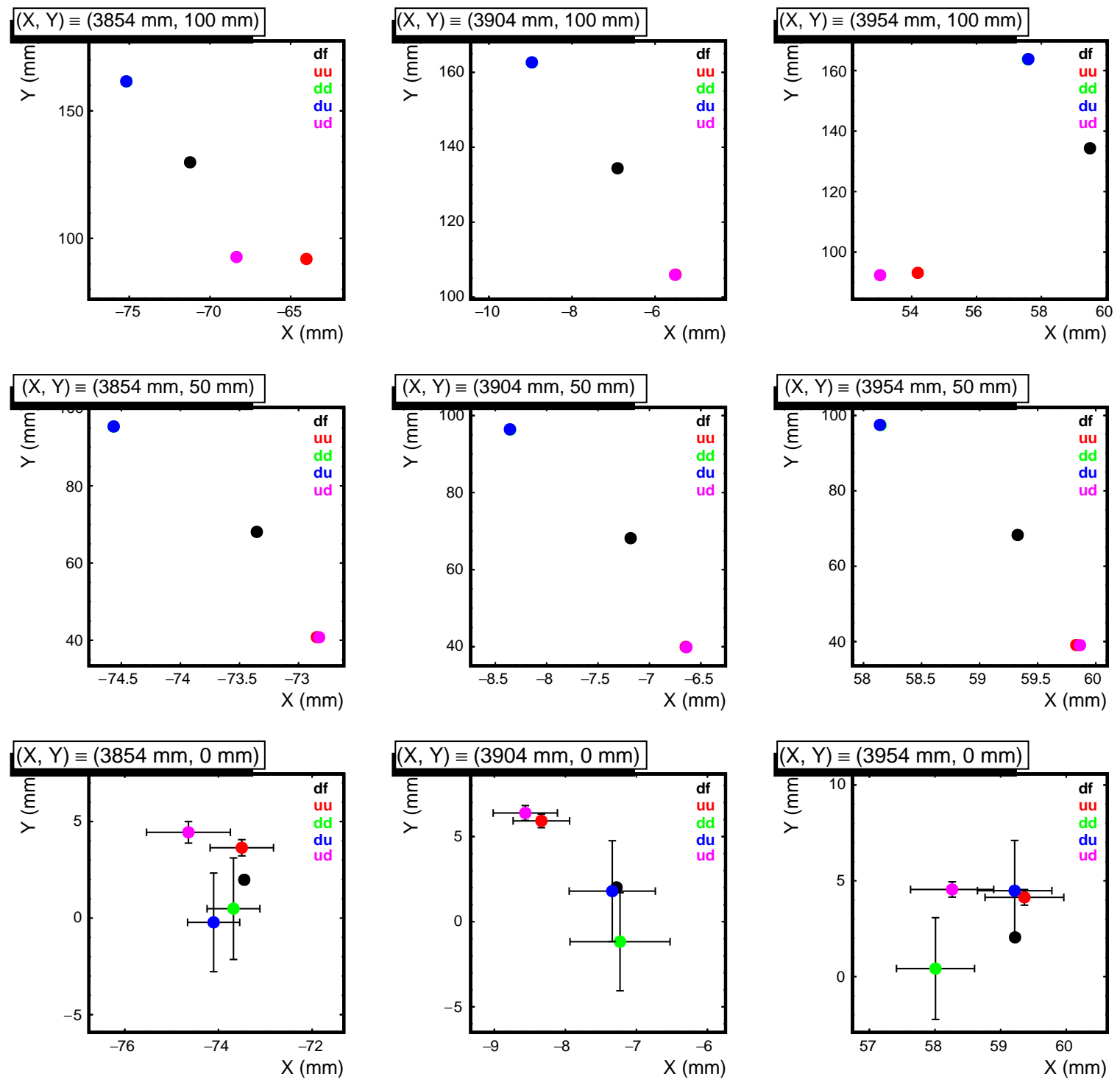

Figure 5-17: Results for $1^{\circ}$ rotation of TS at VD3. In each plot, 5 points are represented: the black point is the default map ( $\mathrm{df}$ in the legend), the red one (uu) is for rotation of TSu about $+X$ axis and TSd about the $-X$ axis, the green one (dd) refers to a rotation of TSu about $-X$ axis and TSd about the $+X$ axis, blue (du) point stands for rotation of both TSu and TSd about $-X$ axis and the pink (ud) point for rotation of both TSu and TSd about $+X$ axis. Points that appear missing are covered by other points. The results include the $4 \sigma$ cut, $\delta$ rays and detector resolution of $300 \mu \mathrm{m}$. Pressure of 1 Torr is assumed. Errors on the $Y$ axis are not visible because of different scales in the $X$ and $Y$ axis. 

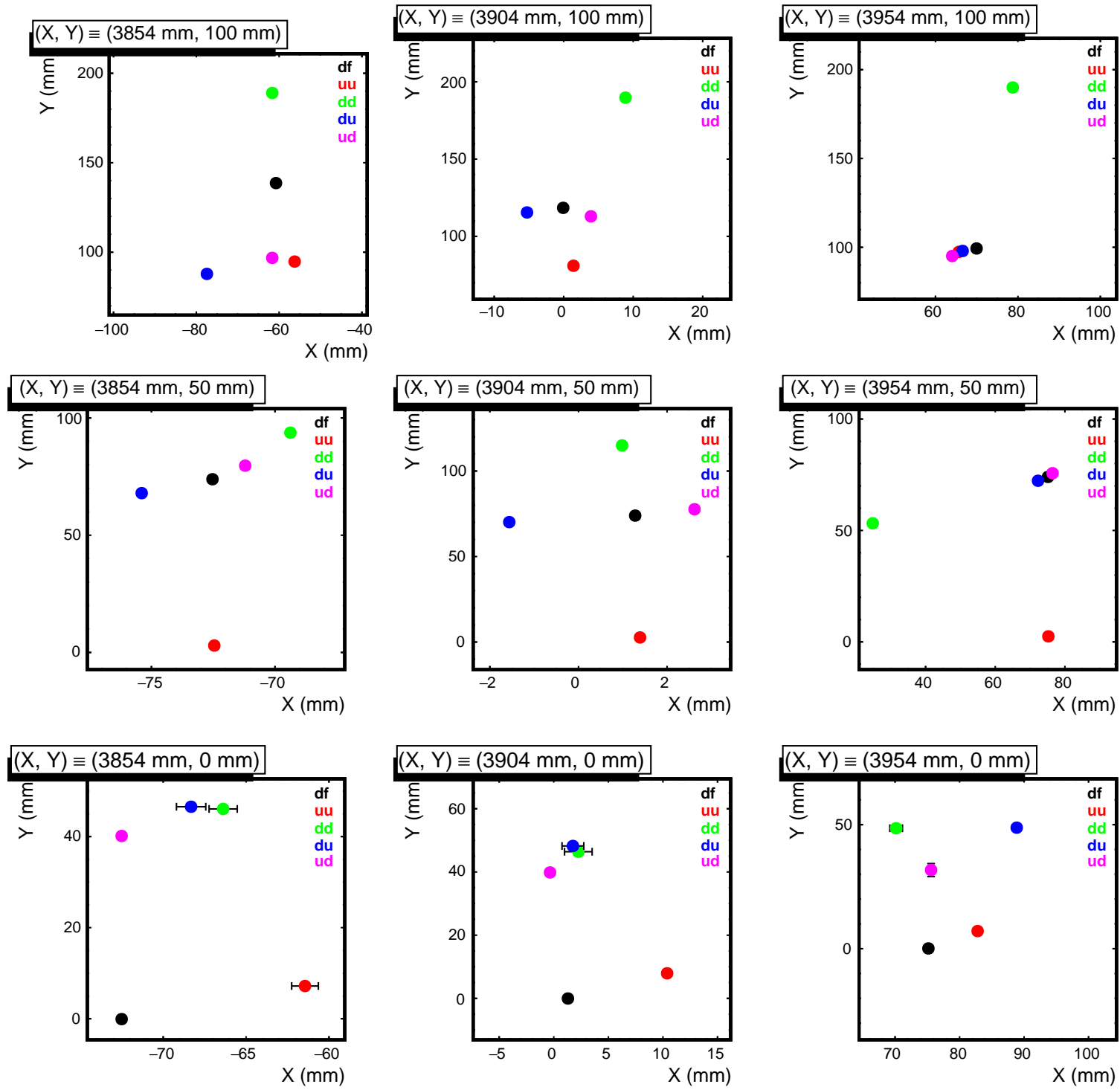

Figure 5-18: Results for $1^{\circ}$ rotation of TS at VD8. In each plot, 5 points are represented: the black point is the default map ( $\mathrm{df}$ in the legend), the red one (uu) is for rotation of TSu about $+X$ axis and TSd about the $-X$ axis, the green one $(\mathrm{dd})$ refers to a rotation of TSu about $-X$ axis and TSd about the $+X$ axis, blue $(\mathrm{du})$ point stands for rotation of both TSu and TSd about $-X$ axis and the pink (ud) point for rotation of both TSu and TSd about $+X$ axis. Points that appear missing are covered by other points. The results include the $4 \sigma$ cut, $\delta$ rays and detector resolution of $300 \mu \mathrm{m}$. Pressure of 1 Torr is assumed. Errors on the $Y$ axis are not visible because of different scales in the $X$ and $Y$ axis. 

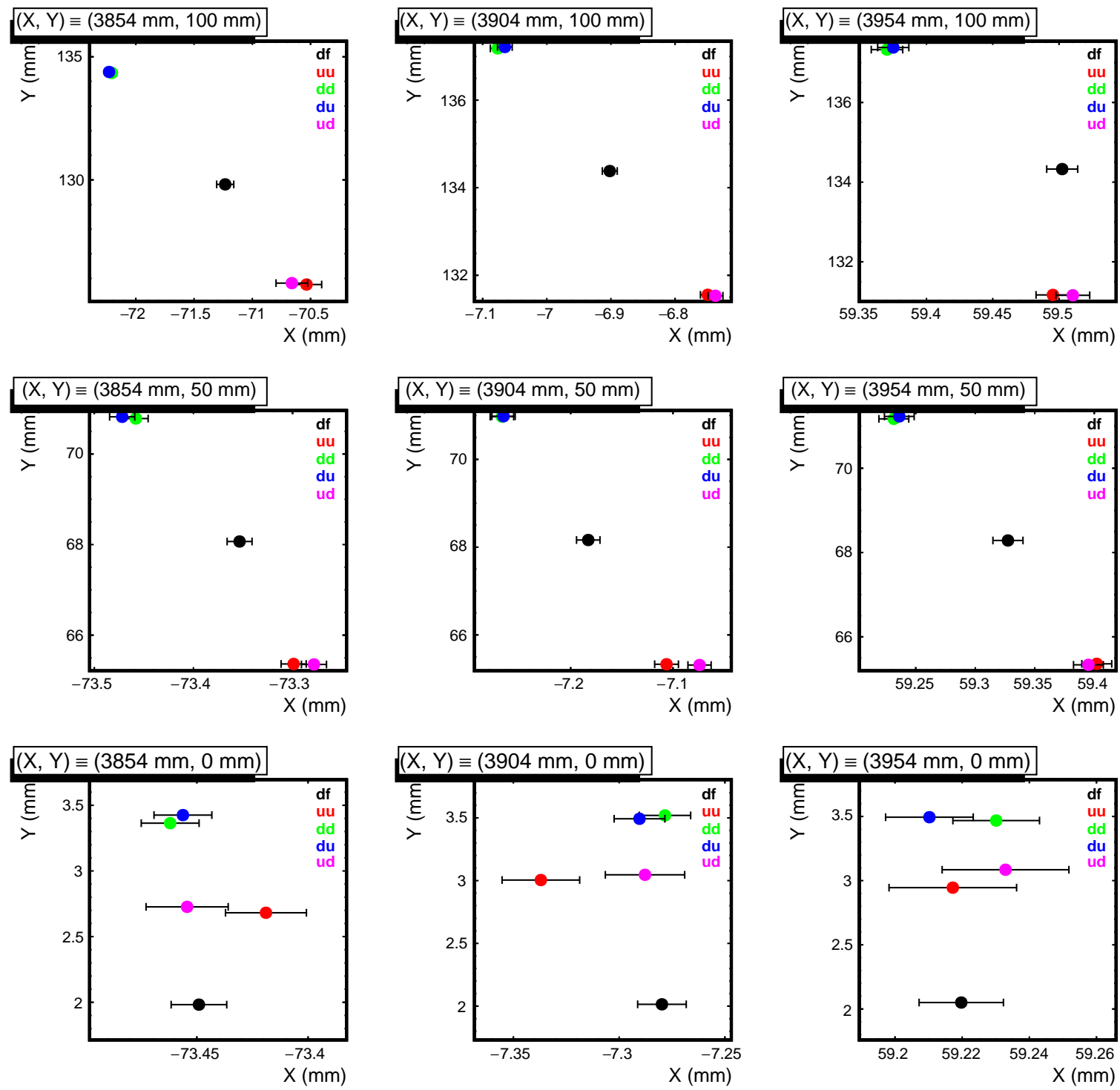

Figure 5-19: Results for $0.1^{\circ}$ rotation of TS at VD3. In each plot, 5 points are represented: the black point is the default map ( $\mathrm{df}$ in the legend), the red one (uu) is for rotation of TSu about $+X$ axis and TSd about the $-X$ axis, the green one (dd) refers to a rotation of TSu about $-X$ axis and TSd about the $+X$ axis, blue (du) point stands for rotation of both TSu and TSd about $-X$ axis and the pink (ud) point for rotation of both TSu and TSd about $+X$ axis. Points that appear missing are covered by other points. The results include the $4 \sigma$ cut, $\delta$ rays and detector resolution of $300 \mu \mathrm{m}$. Pressure of 1 Torr is assumed. Errors on the $Y$ axis are not visible because of different scales in the $X$ and $Y$ axis. 

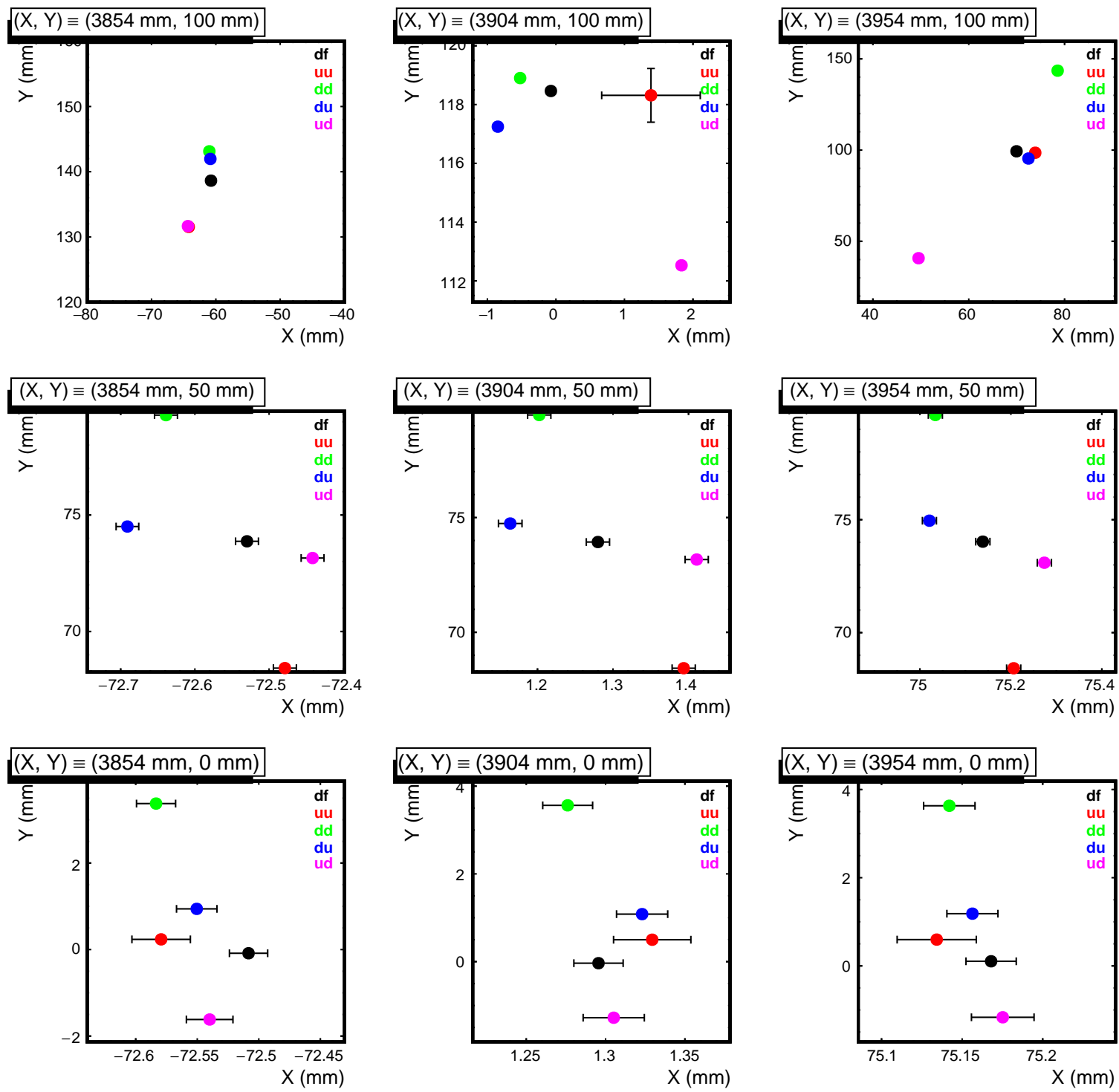

Figure 5-20: Results for $0.1^{\circ}$ rotation of TS at VD8. In each plot, 5 points are represented: the black point is the default map ( $\mathrm{df}$ in the legend), the red one (uu) is for rotation of TSu about $+X$ axis and TSd about the $-X$ axis, the green one $(\mathrm{dd})$ refers to a rotation of TSu about $-X$ axis and TSd about the $+X$ axis, blue $(\mathrm{du})$ point stands for rotation of both TSu and TSd about $-X$ axis and the pink (ud) point for rotation of both TSu and TSd about $+X$ axis. Points that appear missing are covered by other points. The results include the $4 \sigma$ $c u t, \delta$ rays and detector resolution of $300 \mu \mathrm{m}$. Pressure of 1 Torr is assumed. Errors on the $Y$ axis are not visible because of different scales in the $X$ and $Y$ axis. 


\subsubsection{Parallel coil displacements}

Figure 5-21 and Figure 5-22 show results for SolCalc maps with parallel coil displacement of 20 $\mathrm{mm}$ about the $\mathrm{Y}$ axis at VD3 and VD8, respectively. For each plot, 5 points are displayed: the black point corresponds to the default maps, the colored ones correspond to the varied field maps with different TSu and TSd coil displacements. The results include the $4 \sigma$ cut for the outliers, $\delta$ rays (but no $\gamma$ rays) and detector resolution of $300 \mu \mathrm{m}$ in $\mathrm{X}$ and $\mathrm{Y}$. For brevity, the figures show results only for the $\beta$ source moved above the XZ plane in steps of $50 \mathrm{~mm}$. Also in this case, the test seems to be very sensitive to TS misalignments of $\sim 20 \mathrm{~mm}$ and the biggest effects are with respect to to the Y axis.

\subsubsection{Dependence on vacuum conditions}

In order to study the dependence of the test on vacuum conditions, the pressure was varied from 1 Torr to 0.05 Torr and 50 Torr. The Mau10 varied maps for $0.15^{\circ}$ were used, because we are interested in possible variations with the smallest misalignment we can study. The $4 \sigma$ cut for the outliers, $\delta$ rays (but no $\gamma$ rays) and detector resolution are included. Figure 5-23 and Figure 5-24 shows the results at VD3 and VD8, respectively. Table 5.8 and Table 5.9 summarize the results for $\mathrm{X}$ and $\mathrm{Y}$ coordinates at VD3 and VD8, respectively. It results that the test benefits from vacuum: with a vacuum of 1 Torr the test has already sufficient precision to resolve very small TS misalignments, although with 0.05 Torr the error bars become even smaller. 

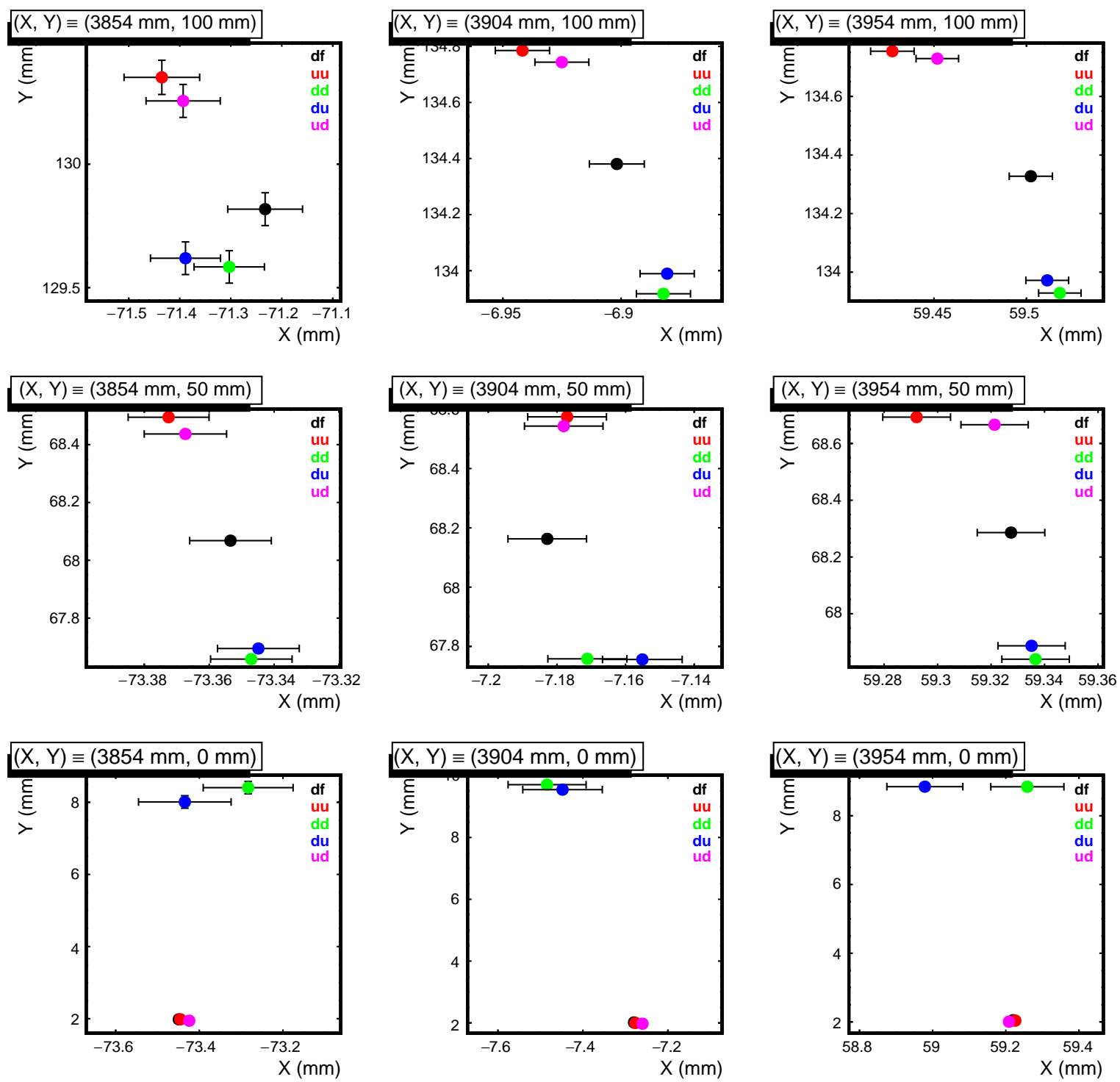

Figure 5-21: Parallel coil shifts with respect to $Y$ axis at VD3. 5 points are represented: the black point is the default map ( $\mathrm{df}$ in the legend), the red one (uu) is for TSu and TSd coils both shifted up, the green one (dd) is for TSu and TSd coils both shifted down, blue (du) stands for TSu coils shifted up and TSd coils shifted down, pink (ud) stands for TSu coils shifted down and TSd coils shifted up. Points that appear missing are covered by other points. The results include the $4 \sigma \mathrm{cut}, \delta$ rays and detector resolution of $300 \mu \mathrm{m}$. Pressure of 1 Torr is assumed. Errors on the $Y$ axis are not visible because of different scales in the $X$ and $Y$ axis. 

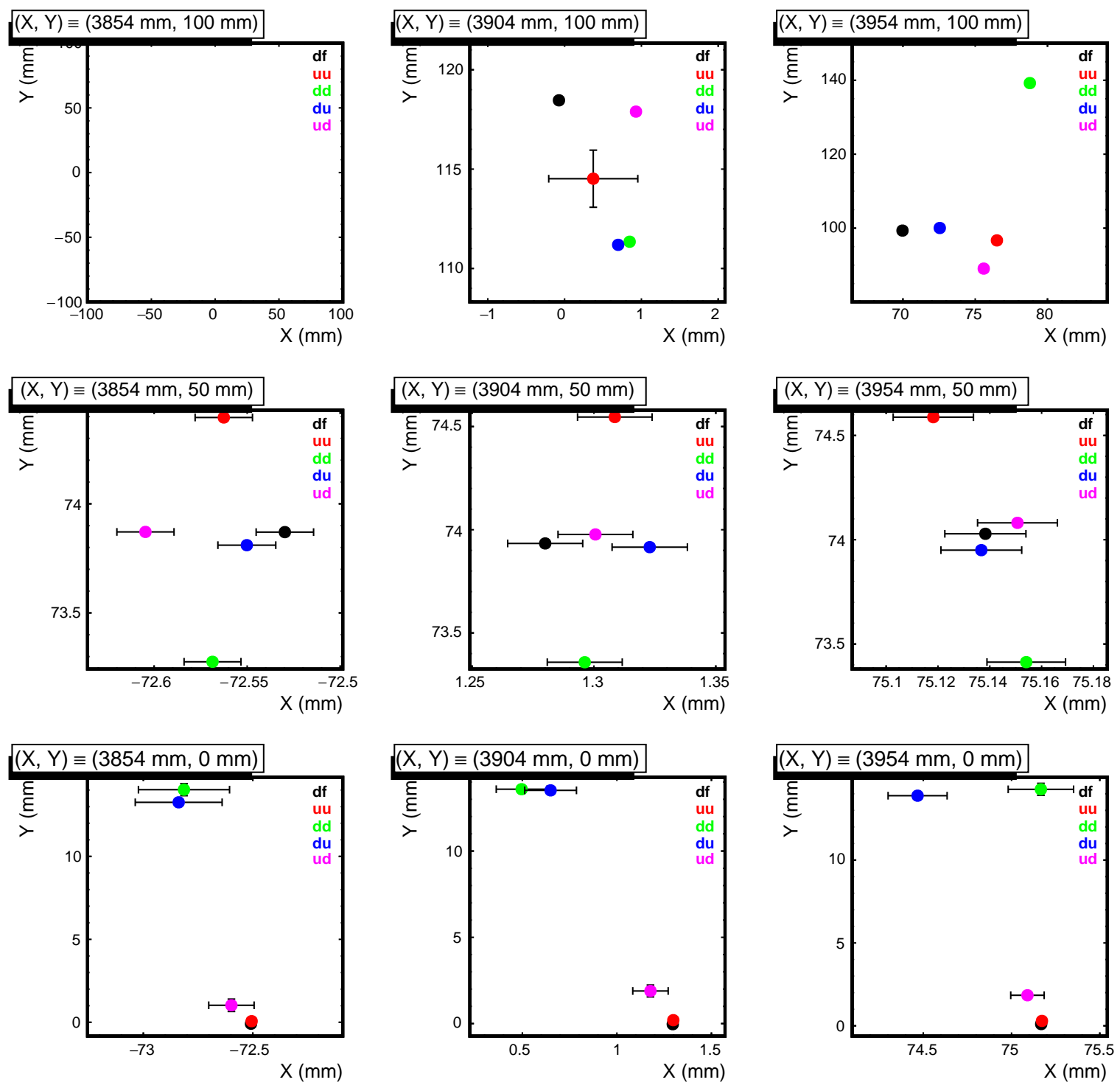

Figure 5-22: Parallel coil shifts with respect to $Y$ axis at VD8. 5 points are represented: the black point is the default map ( $\mathrm{df}$ in the legend), the red one (uu) is for TSu and TSd coils both shifted up, the green one (dd) is for TSu and TSd coils both shifted down, blue (du) stands for TSu coils shifted up and TSd coils shifted down, pink (ud) stands for TSu coils shifted down and TSd coils shifted up. Points that appear missing are covered by other points. The upper left window $((X, Y) \equiv(3854 \mathrm{~mm}, 100 \mathrm{~mm}))$ shows no points because no electrons make it through VD8 in this case. The results include the $4 \sigma \mathrm{cut}, \delta$ rays and detector resolution of $300 \mu m$. Pressure of 1 Torr is assumed. Errors on the $Y$ axis are not visible because of different scales in the $X$ and $Y$ axis. 
Table 5.8: $\beta$ source simulation using Mau10 rotated maps of $0.15^{\circ}$ with respect to $-Z$ axis for $P=1$ Torr, $P=50$ Torr and $P=0.05$ Torr. The results are shown at VD3.

\begin{tabular}{|c|c|c|c|c|c|c|}
\hline \multirow[b]{2}{*}{$(\mathrm{X}[\mathrm{mm}], \mathrm{Y}[\mathrm{mm}])$} & \multicolumn{3}{|c|}{$\bar{x}$} & \multicolumn{3}{|c|}{$\overline{Y Y}$} \\
\hline & $P=1$ Torr & $P=50$ Torr & $\mathrm{P}=0.05$ Torr & $P=1$ Torr & $P=50$ Torr & $P=0.05$ Torr \\
\hline$(3854,0)$ & $-73.834 \pm 0.043$ & $-73.845 \pm 0.072$ & $-73.711 \pm 0.060$ & $7.685 \pm 0.112$ & $4.828 \pm 0.080$ & $6.918 \pm 0.112$ \\
\hline$(3904,0)$ & $-7.622 \pm 0.042$ & $-7.625 \pm 0.069$ & $-7.464 \pm 0.048$ & $7.984 \pm 0.112$ & $5.035 \pm 0.079$ & $6.289 \pm 0.098$ \\
\hline$(3954,0)$ & $58.804 \pm 0.039$ & $58.847 \pm 0.070$ & $58.795 \pm 0.053$ & $7.902 \pm 0.104$ & $5.053 \pm 0.080$ & $6.721 \pm 0.101$ \\
\hline$(3854,50)$ & $-73.702 \pm 0.014$ & $-73.800 \pm 0.061$ & $-73.695 \pm 0.004$ & $66.973 \pm 0.015$ & $68.146 \pm 0.061$ & $66.852 \pm 0.007$ \\
\hline$(3904,50)$ & $-7.551 \pm 0.013$ & $-7.547 \pm 0.058$ & $-7.555 \pm 0.004$ & $67.134 \pm 0.014$ & $68.441 \pm 0.058$ & $66.980 \pm 0.007$ \\
\hline$(3954,50)$ & $58.896 \pm 0.014$ & $58.845 \pm 0.061$ & $58.894 \pm 0.005$ & $67.263 \pm 0.015$ & $68.711 \pm 0.062$ & $67.139 \pm 0.007$ \\
\hline$(3854,100)$ & $-72.849 \pm 0.019$ & $-71.662 \pm 0.090$ & $-73.258 \pm 0.006$ & $131.959 \pm 0.022$ & $131.020 \pm 0.088$ & $132.624 \pm 0.010$ \\
\hline$(3904,100)$ & $-7.307 \pm 0.013$ & $-7.239 \pm 0.058$ & $-7.305 \pm 0.004$ & $133.292 \pm 0.014$ & $134.553 \pm 0.059$ & $133.148 \pm 0.007$ \\
\hline$(3954,100)$ & $59.096 \pm 0.013$ & $58.557 \pm 0.062$ & $59.176 \pm 0.004$ & $133.380 \pm 0.014$ & $133.316 \pm 0.064$ & $133.374 \pm 0.007$ \\
\hline
\end{tabular}
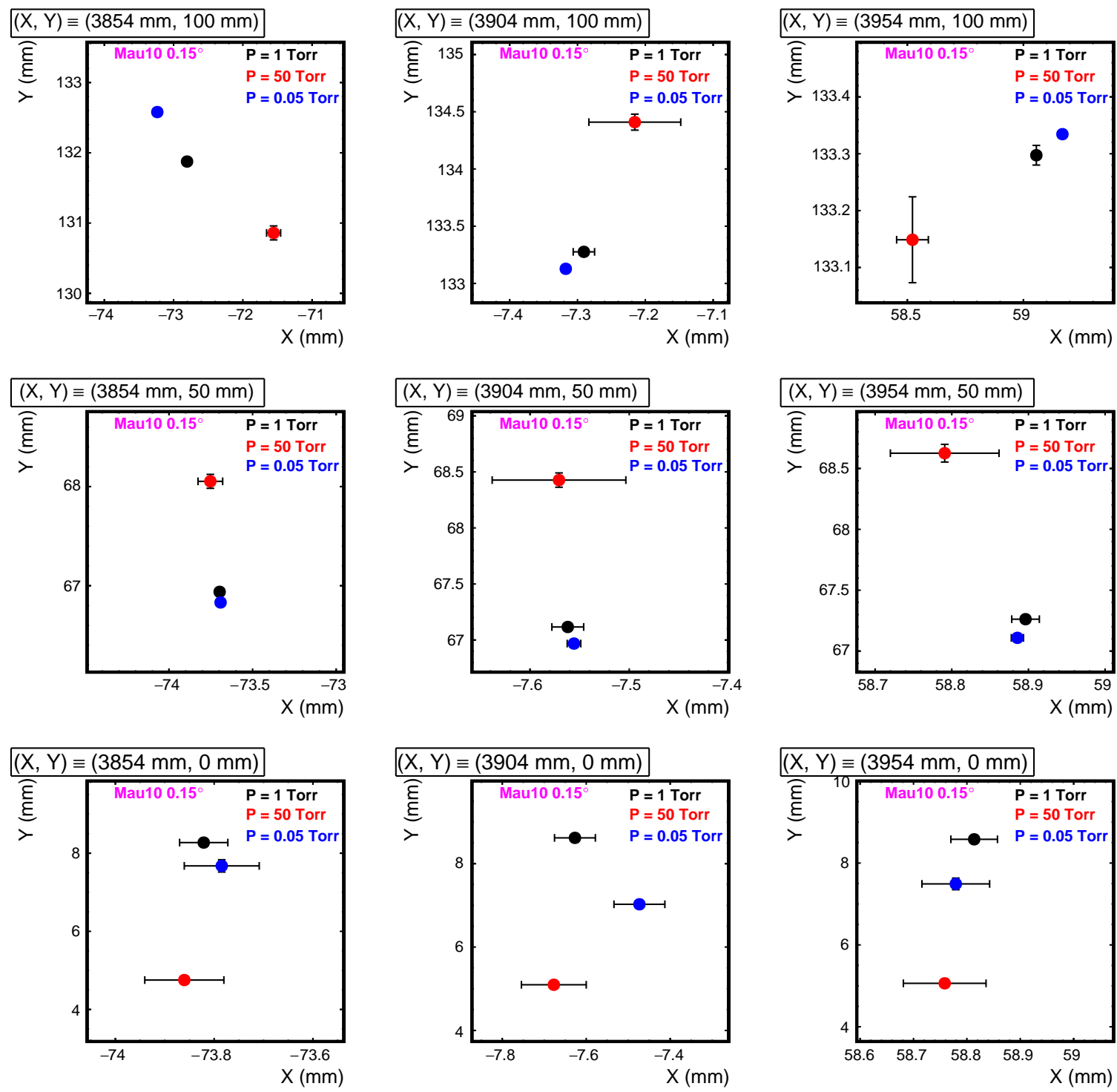

Figure 5-23: $\beta$ source simulation using Mau10 rotated maps of $0.15^{\circ}$ with respect to $-Z$ axis at VD3. Errors on the $Y$ axis are not visible because of different scales in the $X$ and $Y$ axis. 
Table 5.9: $\beta$ source simulation using Mau10 rotated maps of $0.15^{\circ}$ with respect to $-Z$ axis for $P=1$ Torr, $P=50$ Torr and $P=0.05$ Torr. The results are shown at VD8.

\begin{tabular}{|c|c|c|c|c|c|c|}
\hline \multirow[b]{2}{*}{$(\mathrm{X}[\mathrm{mm}], \mathrm{Y}[\mathrm{mm}])$} & \multicolumn{3}{|c|}{$x$} & \multicolumn{3}{|c|}{$\mathrm{Y}$} \\
\hline & $P=1$ Torr & $P=50$ Torr & $\mathrm{P}=0.05$ Torr & $P=1$ Torr & $P=50$ Torr & $\mathrm{P}=0.05$ Torr \\
\hline$(3854,0)$ & $-72.265 \pm 0.040$ & $-72.184 \pm 0.118$ & $-72.190 \pm 0.041$ & $13.374 \pm 0.232$ & $5.269 \pm 0.122$ & $6.209 \pm 0.086$ \\
\hline$(3904,0)$ & $1.420 \pm 0.042$ & $1.494 \pm 0.116$ & $1.489 \pm 0.037$ & $12.517 \pm 0.218$ & $5.632 \pm 0.122$ & $6.061 \pm 0.085$ \\
\hline$(3954,0)$ & $74.967 \pm 0.037$ & $74.980 \pm 0.116$ & $74.993 \pm 0.036$ & $12.207 \pm 0.206$ & $5.632 \pm 0.116$ & $5.667 \pm 0.086$ \\
\hline$(3854,50)$ & $-72.211 \pm 0.016$ & $-71.922 \pm 0.096$ & $-72.244 \pm 0.008$ & $74.804 \pm 0.017$ & $74.402 \pm 0.098$ & $74.900 \pm 0.008$ \\
\hline$(3904,50)$ & $1.352 \pm 0.016$ & $1.381 \pm 0.096$ & $1.350 \pm 0.008$ & $74.933 \pm 0.017$ & $75.072 \pm 0.099$ & $74.970 \pm 0.009$ \\
\hline$(3954,50)$ & $74.950 \pm 0.016$ & $74.420 \pm 0.096$ & $74.955 \pm 0.008$ & $75.013 \pm 0.017$ & $74.473 \pm 0.098$ & $75.061 \pm 0.009$ \\
\hline$(3854,100)$ & $-73.611 \pm 1.905$ & $-56.039 \pm 7.241$ & $0.000 \pm 0$ & $98.549 \pm 1.942$ & $97.899 \pm 3.780$ & $0 \pm 0$ \\
\hline$(3904,100)$ & $-2.523 \pm 2.651$ & $-0.788 \pm 1.451$ & $7.027 \pm 9.394$ & $101.096 \pm 6.930$ & $116.794 \pm 0.927$ & $73.960 \pm 21.311$ \\
\hline$(3954,100)$ & $-56.826 \pm 66.057$ & $-2.970 \pm 43.071$ & $-93.981 \pm 14.416$ & $-21.058 \pm 50.550$ & $58.500 \pm 46.914$ & $-47.506 \pm 27.485$ \\
\hline
\end{tabular}
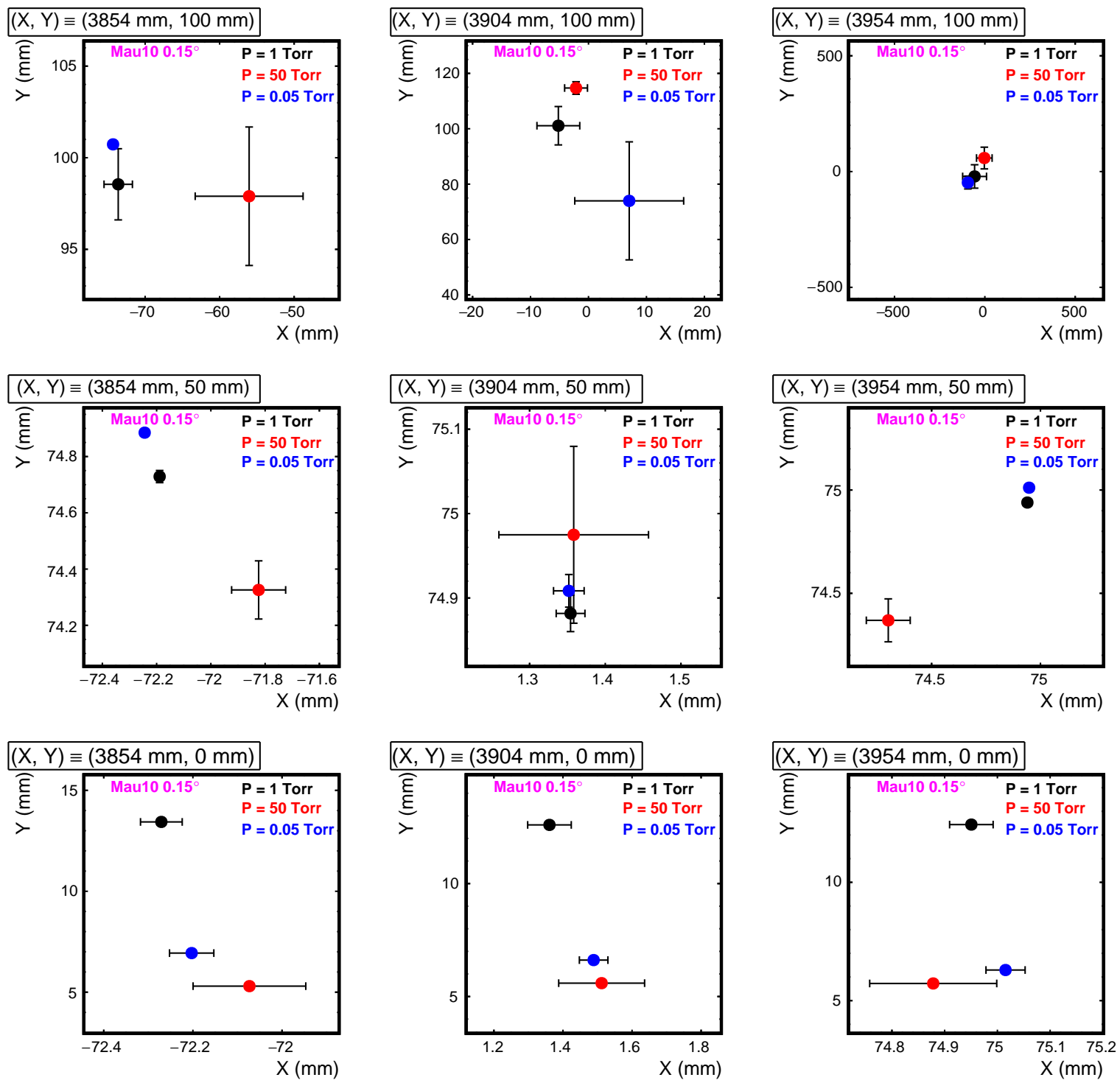

Figure 5-24: $\beta$ source simulation using Mau10 rotated maps of $0.15^{\circ}$ with respect to $-Z$ axis at VD8. Errors on the $Y$ axis are not visible because of different scales in the $X$ and $Y$ axis. 


\subsection{NeXT STEPS}

The study of the low-momentum electron test of the TS presented in this thesis provides a proof of principle of the test: it demonstrates that, under realistic conditions, it is sensitive to misalignments down to the order of a few $\mathrm{mm}$ and can quantify the corresponding effects proportionally to the misalignment amplitude, even though it cannot discriminate among various types of misalignments. However, prior to a final decision on its integration to the program of operations of the experiment, the following points need further study:

1. More cases of misalignment; e.g. horizontal rotations about the $\mathrm{Y}$ axis. The vertical rotations about the $\mathrm{X}$ and $\mathrm{Z}$ axes examined here are the most important for the functioning of TS, but they do not provide a complete exploration of all basic rotations.

2. Medium effects in a finite-size source.

3. A realistic detector model.

4. The requirements on the source and detector alignment, by folding the uncertainties of corresponding misalignments to the results for the mean transverse coordinates of the detected electrons. In leading order, these can be added linearly to the statistical uncertainties but, for a more precise analysis, simulations with misaligned source and detector should be done.

5. An estimation of effort and cost of the test and a deployment plan. It is recommended to do the test first prior to the operations and then in every downtime, in order to ensure the TS survey and cross-check among running periods. 


\section{CHAPTER 6}

\section{CONCLUSIONS}

This thesis aimed to understand the impact of field uncertainties in the Transport Solenoid on the physics parameters of the Mu2e experiment. Uncertainties in the TS field arising from misalignments of the coils due to mechanical and magnetic forces were examined. The study sought to find whether the field misalignments may have effects on the muon and pion stopping rates on the target and on the beam electron background yield. Since the TS field uncertainties can potentially affect the physics parameters of the experiment, while at the same time it is mechanically difficult to measure this field, there is need for a method to test TS performance prior to the operations. The proposed method is the $\beta$ source test, because of its ability to approximately trace the field lines with low momentum electrons and thus detect any unwanted distortions of the field due to misalignments. The two lines of study are coherent: the more important the consequences of TS field uncertainties to the physics parameters of the experiment, the more sensitive the $\beta$ source test has to be. In other words, the sensitivity of the physics parameters to TS misalignments drives the specifications of the $\beta$ source test regarding low momentum electron detector resolution, vacuum conditions, presence of antiproton absorption windows and/or collimators, locations of the source and the detector, etc. This study included a detailed validation of different solenoid designs, including the comparisons between different coil models (split coils compared to helical winding).

Changes in the muon and pion stopping rates may have impact on the physics parameters of the Mu2e experiment, since they can change proportionally the rates of both signal and scatteringinduced background. Moreover, variations in the timing and shapes of the stopped particle distributions can change the acceptance of the signal and of the backgrounds. The distribution of high momentum electrons arriving at the $\mathrm{Al}$ target depends on the TS field. Field misalignments can change the number of high momentum electrons produced in the stopping target, by steering mother particles differently into various scatterers along the transport line. Also, field misalignments 
can steer more electrons to the stopping target and then to the detector for the same number of beam electrons. Variations of the TS field due to misalignments specify the desired sensitivity of the $\beta$ source test, in which collimated, low momentum electrons are sent into TS and traced using a detector located at various positions along TS.

To study the field uncertainties, rotations and parallel coil displacements were introduced in the default field: the misalignments corresponding to $0.50^{\circ}$ rotation about the $-\mathrm{Z}$ axis (Mau10), $1^{\circ}$ rotation about the $X$ axis (SolCalc) and coil shifts of $20 \mathrm{~mm}$ parallel to the $Y$ axis are very unlikely to happen with the present engineering systems for alignment of large devices. They were studied in order to make sure that the effects on the rates, backgrounds and $\beta$ source test were noticeable. The misalignment amplitude was then reduced in order to reproduce cases that can occur in reality, although still rather unlikely, such as those corresponding to $0.15^{\circ}$ rotation with respect to the $-\mathrm{Z}$ axis (Mau10) and $0.10^{\circ}$ rotation about the $\mathrm{X}$ axis (SolCalc). The misalignments that have been studied are rigid-body rotations of TS, because these have the largest effect on individual tracks and are also the most likely to happen through contraction and magnetic stress on the joint support system of the coils after cooling and powering up the solenoid. The rotations considered in this study are only field rotations: all material elements in the Mu2e geometry (including collimators, vacuum bores, cryostats, and the coils themselves) are left in-place in the simulations.

The effect of realistic variations on the muon and pion stopping rates is only on the normalization factor: the fractional yield difference with respect to the default field is of the order of $\sim 2 \%$. For bigger variations, besides the statistics, also the shape of the position distributions of muons and pions in the stopping target changes. Depending on the kind of rotation or coil displacement, the spatial distribution results substantially differ with respect to the default magnetic field. This effect is evident especially for pions. The overall normalization of the muon capture rate reflects on the integral of both the signal and the background. The background rates which are correlated with the muon capture rate (i.e. that scale with beam intensity), such as DIO, RMC and RPC, have to be scaled with the fractional yield differences created by the misalignments. This represents a systematic uncertainty, but can be applied only for those cases which retain the same shapes, in other words they do not change the signal and background acceptance. If the position and time distributions change, an estimate of the new acceptance and the new fraction making it into the live time window are required. It has been found that the distribution shapes are not affected by misalignments of realistic size, therefore there is no such need recommended. Consequently, in order to determine the uncertainties on the signal and background from field misalignments, the current estimates can be simply scaled within the normalization variations induced by the field variations.

The present study has shown that the beam electron background with various field configurations, including TS misalignments, is very small: the expected yield is $<5 \times 10^{-4}$ at $90 \%$ C.L. 
This study has yielded estimates of this background systematically higher than the older study. This change is attributed to different methods of simulating electron creation along the beam transport line: in the present analysis, electrons are created through the entire transport line to the stopping target, whereas in the previous analysis they are created in the upstream half of the transport line. The difference in the analyzed region of electron origin results in a larger number of electrons arriving at the capture target and with a larger spread in the $Y$ dimension, thus scattering more electrons off the capture target material, which subsequently hit the tracker. The method applied in the present analysis covers partly the delayed background that is classified in the TDR [Mu2e Collaboration(2015)] as "decays in flight" (DIF), coming from pions and muons that decay producing high momentum electrons inside DS. The fraction of the DIF background included in the present analysis accounts for pion and muon decays in the region of DS between the entrance and the stopping target. This work suggests that the new definition of the region of origin of high-momentum electrons is more concise and realistic, and thus the new number is proposed to be adopted as the official estimate of this background. The study indicates that the background from beam electrons, including part of DIF, is essentially insensitive to realistic TS misalignments and remains one of the minor backgrounds of the experiment.

The $\beta$ source test resolution is pretty high with a mild vacuum and a good detector. The test benefits from vacuum: with 1 Torr, the test has already sufficient precision to resolve very small TS misalignments. A mild vacuum is the best choice for this experiment, since it has to be conducted before the positioning of the TS1 $\bar{p}$ absorber and the cooling. This test appears to resolve misalignments down to the level of a tenth of the degree or less (equivalent to a few millimeters maximum coil displacement), if the vacuum is moderately low (of the order of 1 Torr) and the detector spatial resolution is sufficiently high (at the sub-millimeter level). If the muon and pion rates and the beam electron background were sensitive to the field uncertainties, the $\beta$ source test would thus be a powerful probe to detect and correct dangerous misalignments, before cooling and powering the solenoids. In this thesis, these physics parameters of the experiment were not found significantly sensitive to realistic misalignments. Nevertheless, the $\beta$ source test remains a valuable test to detect any possible field uncertainties, even if it is not able to discriminate what kind of misalignment has occurred. To maintain its power, a sub-millimeter resolution detector is required. A suitable solution appears to be a fiber tracker, with a resolution of the order of $300 \mu \mathrm{m}$. The measurement of the signal amplitude is not required, since only the position in the plane transverse to the transport line needs to be measured. A realistic detector model will need to be simulated in order to establish the best choice for the $\beta$ source test. Further steps along this line of study, which will allow making a decision on the need and functionality of the $\beta$ source test, include optimization of the detector model, examination of systematic effects from source and detector misalignments, medium effects in a finite-size source and an estimation of effort and cost 
of the test along with a deployment plan. 


\section{BIBLIOGRAPHY}

[Paschos(2007)] E. A. Paschos, Electroweak Theory (Cambridge University Press, 2007), ISBN 9780511611049, cambridge Books Online, URL http://dx.doi.org/10.1017/ CB09780511611049.

[Marciano et al.(2008)] W. J. Marciano et al., Annual Review of Nuclear and Particle Science 58, 315 (2008), http://dx.doi.org/10.1146/annurev.nucl.58.110707.171126, URL http: //dx.doi.org/10.1146/annurev.nucl.58.110707.171126.

[Beringer et al.(2012)] J. Beringer et al. (Particle Data Group), Phys. Rev. D 86, 010001 (2012), URL http://link.aps.org/doi/10.1103/PhysRevD.86.010001.

[Raidal et al.(2008)] M. Raidal et al., Eur. Phys. J. C57, 13 (2008), 0801.1826.

[Baldini et al.(2016)] A. M. Baldini et al. (MEG) (2016), 1605. 05081.

[Bellgardt et al.(1988)] U. Bellgardt et al., Nuclear Physics B 299, 1 (1988), ISSN 0550-3213, URL http://www. sciencedirect.com/science/article/pii/0550321388904622.

[Bertl et al.(2006)] W. H. Bertl et al. (SINDRUM II), Eur. Phys. J. C47, 337 (2006).

[Bernstein and Cooper(2013)] R. H. Bernstein and P. S. Cooper, Phys. Rept. 532, 27 (2013).

[de Gouvea and Vogel(2013)] A. de Gouvea and P. Vogel, Prog. Part. Nucl. Phys. 71, 75 (2013), 1303.4097.

[Cei and Nicolò(2014)] F. Cei and D. Nicolò, Advances in High Energy Physics 2014, 31 (2014).

[Baldini et al.(2013)] A. M. Baldini et al. (2013), 1301.7225.

[Dohmen et al.(1993)] C. Dohmen et al., Physics Letters B 317, 631 (1993), ISSN 0370-2693, URL http://www. sciencedirect. com/science/article/pii/037026939391383X.

[Jackson(1999)] J. D. Jackson, Classical electrodynamics (Wiley, New York, NY, 1999), 3rd ed., ISBN 9780471309321, URL http://cdsweb. cern.ch/record/490457. 
[Agostinelli et al.(2003)] S. Agostinelli et al., Nuclear Instruments and Methods in Physics Research Section A: Accelerators, Spectrometers, Detectors and Associated Equipment 506, 250 (2003), ISSN 0168-9002, URL http://www.sciencedirect.com/science/article/pii/ S0168900203013688.

[Brun and Rademakers(1997)] R. Brun and F. Rademakers, Nuclear Instruments and Methods in Physics Research Section A: Accelerators, Spectrometers, Detectors and Associated Equipment 389, 81 (1997), ISSN 0168-9002, new Computing Techniques in Physics Research V, URL http://www.sciencedirect.com/science/article/pii/S016890029700048X.

[Fields(2012)] C. Fields, Opera 3d version 15 r13 (2012), URL http://cobham.vectorfields .com/.

[Bartoszek et al.(2014)] L. Bartoszek et al. (Mu2e) (2014), 1501.05241.

[MathWorks $\left.{ }^{\circledR}(2015 b)\right]$ MathWorks ${ }^{\circledR}, \operatorname{MATLAB}$ (2015b), URL http://www . mathworks . com/.

[Mu2e Collaboration(2015)] Mu2e Collaboration, Mu2e Technical Design Report (arXiv:1501.05241, 2015).

[Landau(1944)] L. Landau, J. Phys.(USSR) 8, 201 (1944).

[Vavilov(1957)] P. V. Vavilov, Sov. Phys. JETP 5, 749 (1957), [Zh. Eksp. Teor. Fiz.32,920(1957)].

[hei(1954)] The Quantum Theory of Radiation, Monographs on Physics (Oxford University Press, 1954).

[Bethe(1953)] H. A. Bethe, Phys. Rev. 89, 1256 (1953), URL http://link.aps.org/doi/10.1103/ PhysRev.89.1256.

[CSDA(2015)] CSDA, Continuous slowing down approximation (2015), URL http://physics.nist. gov/.

[Logue and Chern(1968)] L. J. Logue and B. Chern, Phys. Rev. 175, 1367 (1968).

[Krane and Halliday(1987)] K. S. Krane and D. Halliday, Introductory nuclear physics (Wiley, 1987).

[Ruchti(1996)] R. C. Ruchti, Annual Review of Nuclear and Particle Science 46, 281 (1996), http: // dx.doi.org/10.1146/annurev. nucl.46.1.281, URL http://dx.doi.org/10.1146/annurev . nucl.46.1.281.

[Coutrakon et al.(2014)] G. Coutrakon et al. (2014), 1409.0049, URL http://inspirehep.net/ record/1313179/files/arXiv:1409.0049.pdf. 